Supporting Information

\title{
Mechanistic Understanding of the Pd(0)-Catalyzed Coupling Cyclization of 1,2-Allenyl Ketones with Aryl Halides: A Computational Study
}

\author{
Kang Lv, ${ }^{\dagger}, *$ Ping Dai, ${ }^{\dagger}$ Xiaoguang Bao*,† \\ ${ }^{\dagger}$ College of Chemistry, Chemical Engineering and Materials Science, Soochow University, 199 \\ Ren-Ai Road, Suzhou Industrial Park, Suzhou, Jiangsu 215123, China. \\ ${ }^{\ddagger}$ Department of Chemistry and Chemical Engineering, Jining University, Qufu, Shandong 273155 , \\ China. \\ E-mail: xgbao@suda.edu.cn

\section{Contents}

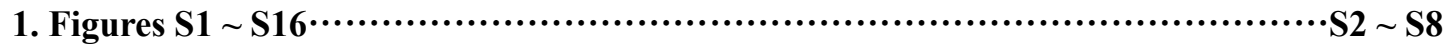

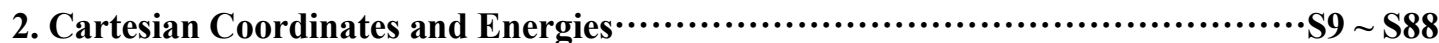




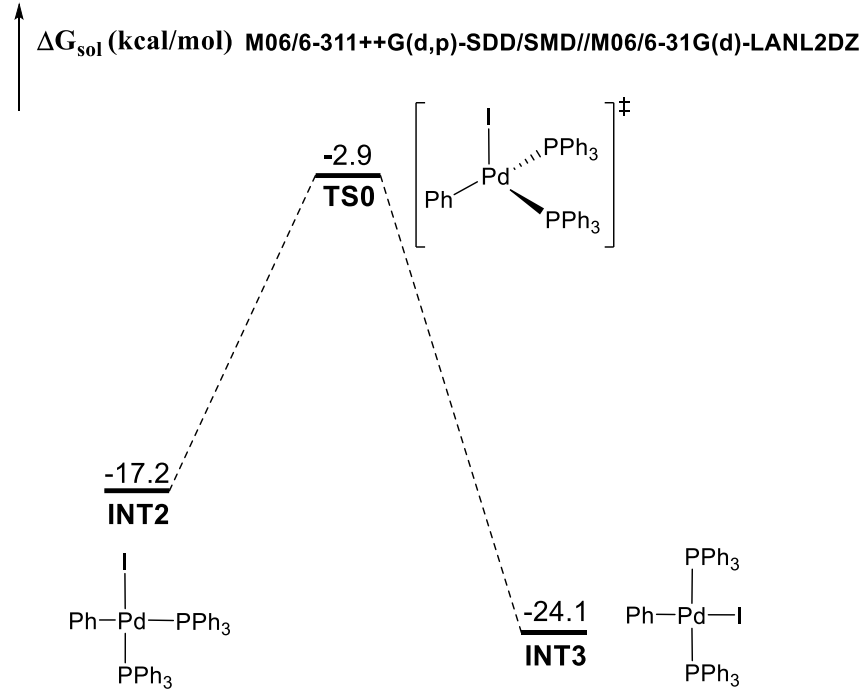

Figure S1. Energy profile for the isomerization from the cis (INT2) to trans (INT3) structure.

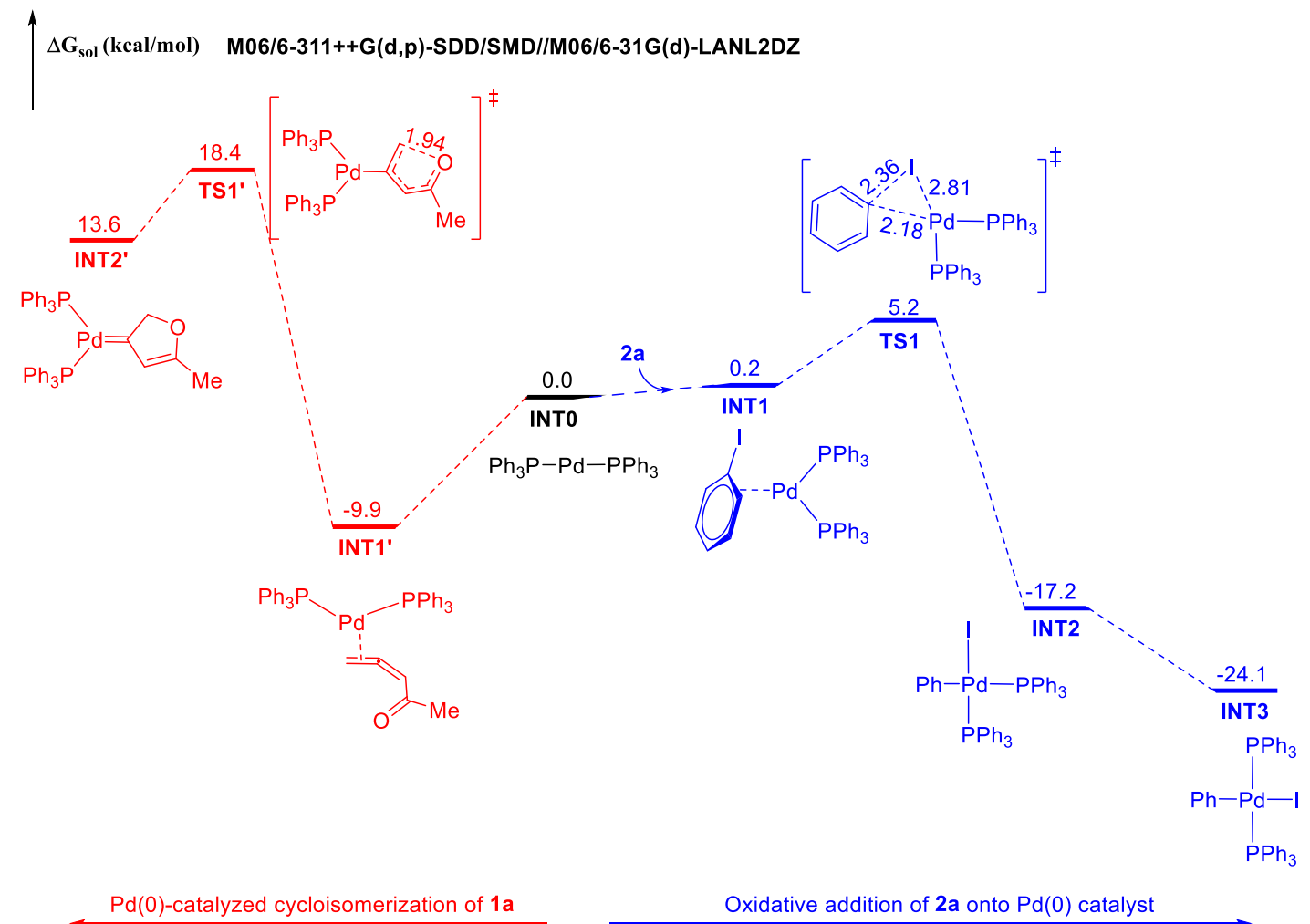

Figure S2. Energy profiles for the oxidative addition of $\mathbf{2 a}$ onto the $\operatorname{Pd}(0)$ catalyst and the $\operatorname{Pd}(0)$-catalyzed cycloisomerization of $\mathbf{1 a}$. 


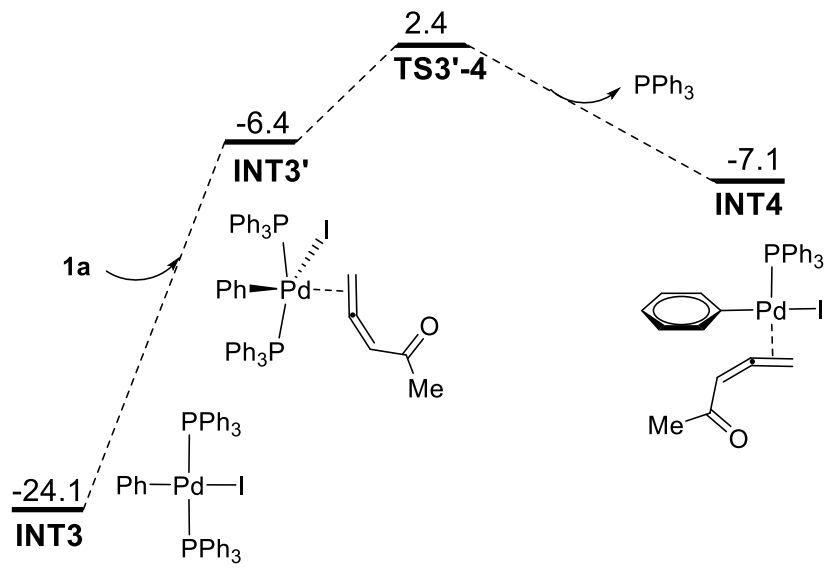

Figure S3. Energy profile for the transformation from INT3 to INT4.

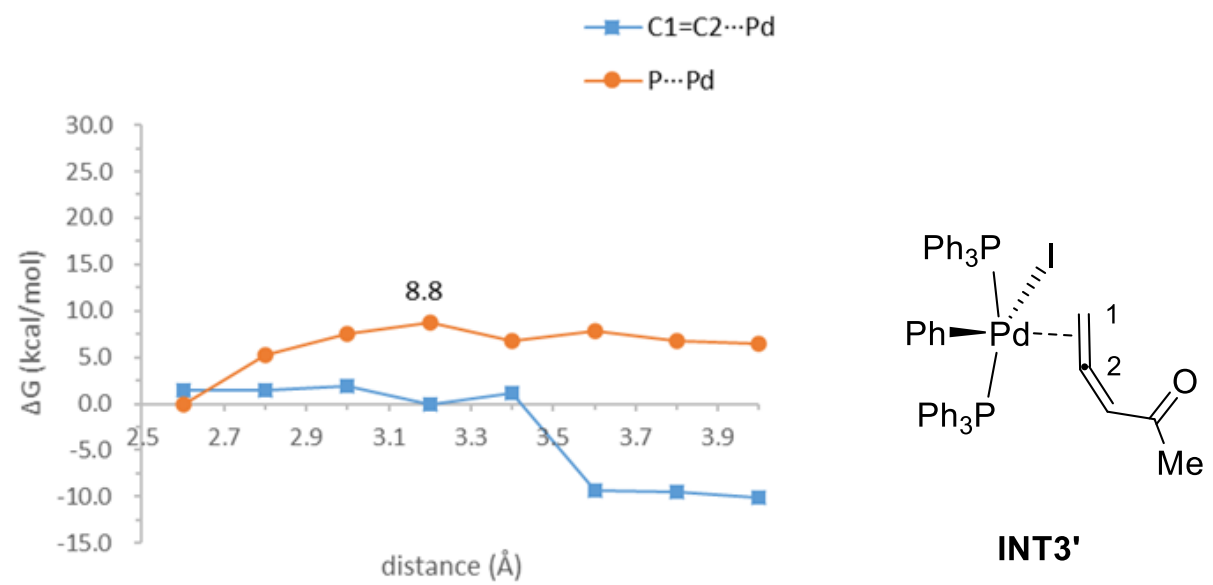

Figure S4. Energy profiles of the potential energy surface scan for the dissociation of the allene substrate and the $\mathrm{PPh}_{3}$ ligand for INT3', respectively.

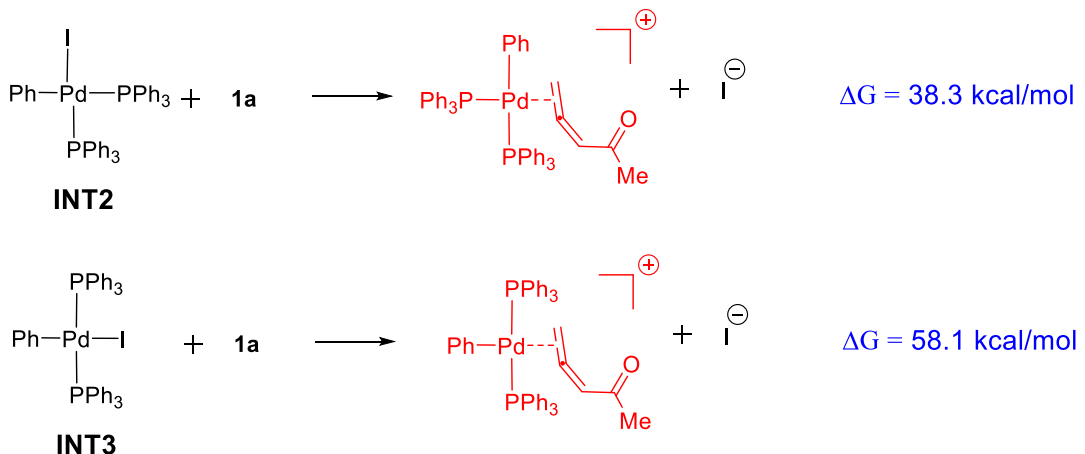

Figure S5. The thermodynamical properties for the replacement of iodine anion in INT2 and INT3 with 1 a. 


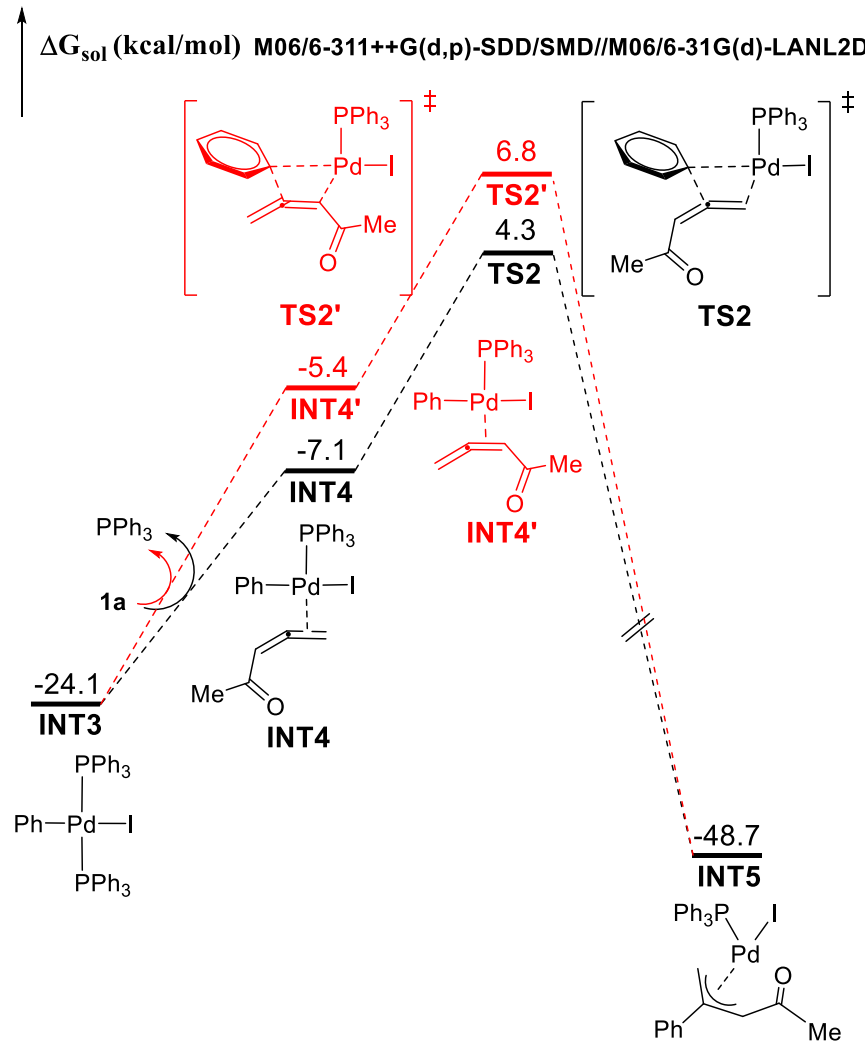

Figure S6. Energy profiles for the formation of $\pi$-allyl-Pd(II) intermediate via TS2 and TS2'.
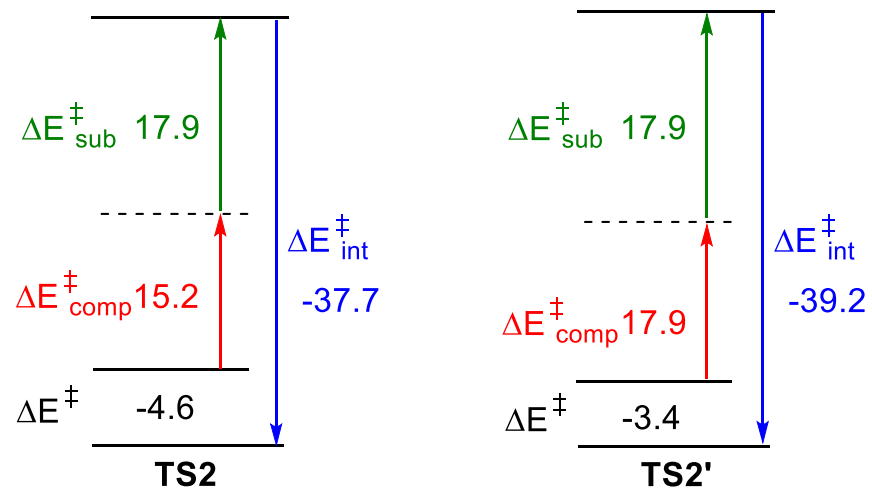

Figure S7. Distortion, interaction and activation energies for TS2 and TS2' (green arrow: distortion energy of 1a; red arrow: distortion energy of the organometallic moiety; blue arrow: interaction energy; black: activation energy, in $\mathrm{kcal} / \mathrm{mol}$ ).

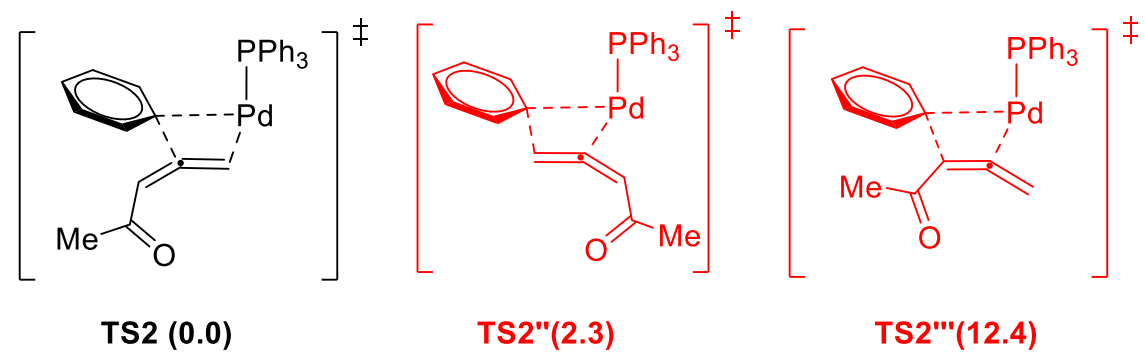

Figure S8. Energy comparisons between TS2 and the other two unfavorable transition states of migratory insertion of $\mathrm{Ph}$ group. The numbers in parenthesis are $\Delta \Delta \mathrm{G}^{\neq}($in $\mathrm{kcal} / \mathrm{mol})$. 


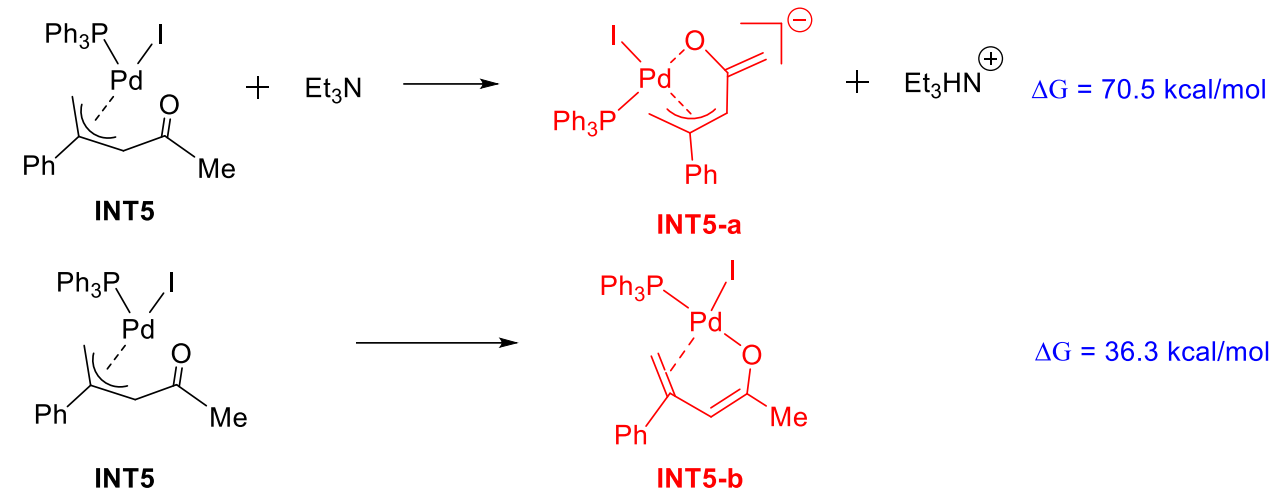

Figure S9. The thermodynamical properties for the paths II- $b$ and II-c shown in Scheme 2.

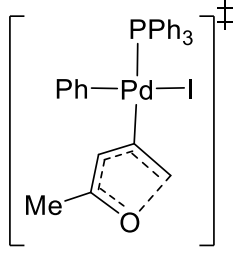

TS6 (0.0)

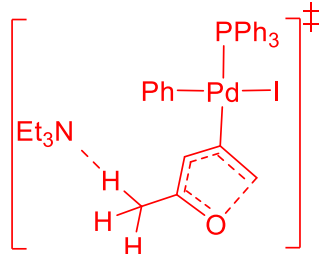

TS6' (3.1)

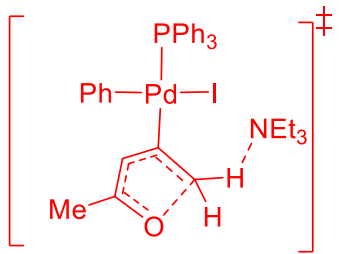

TS6" (0.9)

Figure S10. Comparisons of the pathways via TS6 and other two unfavorable transition states of intramolecular nucleophilic cyclization in the presence of the base $\mathrm{Et}_{3} \mathrm{~N}$. The numbers shown in parenthesis are $\Delta \Delta \mathrm{G}^{\neq}$(in $\mathrm{kcal} / \mathrm{mol}$ ).
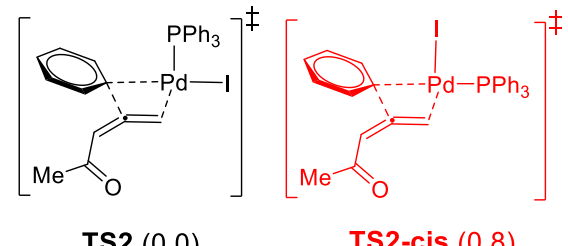

TS2-cis (0.8)

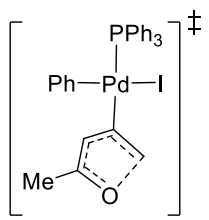

TS6 (0.0)

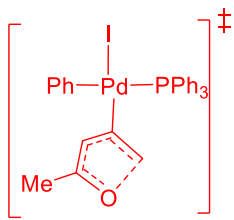

TS6-cis-1 (6.9)

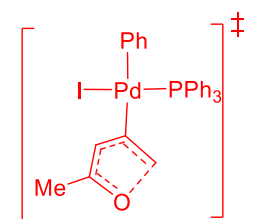

TS6-cis-2 (6.1)

Figure S11. Comparisons between the different structural conformer(s) for TS2 and TS6, respectively. The relative Gibbs energies (in $\mathrm{kcal} / \mathrm{mol}$ ) are given in parenthesis. 


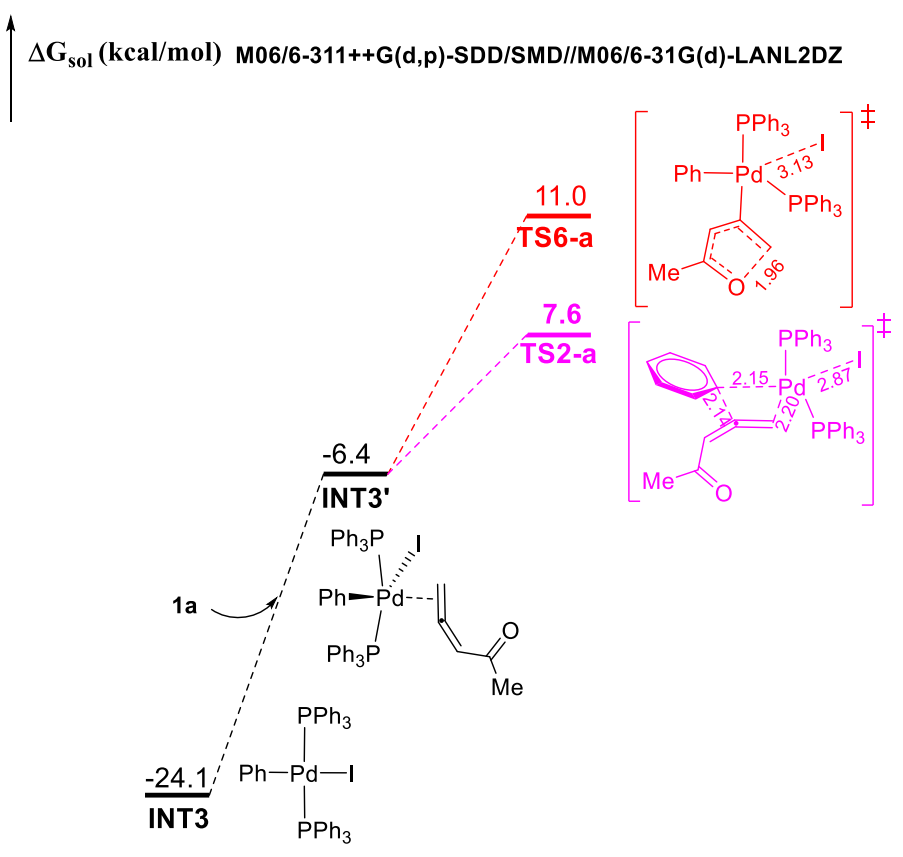

Figure S12. Energy profiles for the two key pathways via $\mathbf{I N T 3}^{\prime}$, in which an ion pair is considered for the two key TS structures.

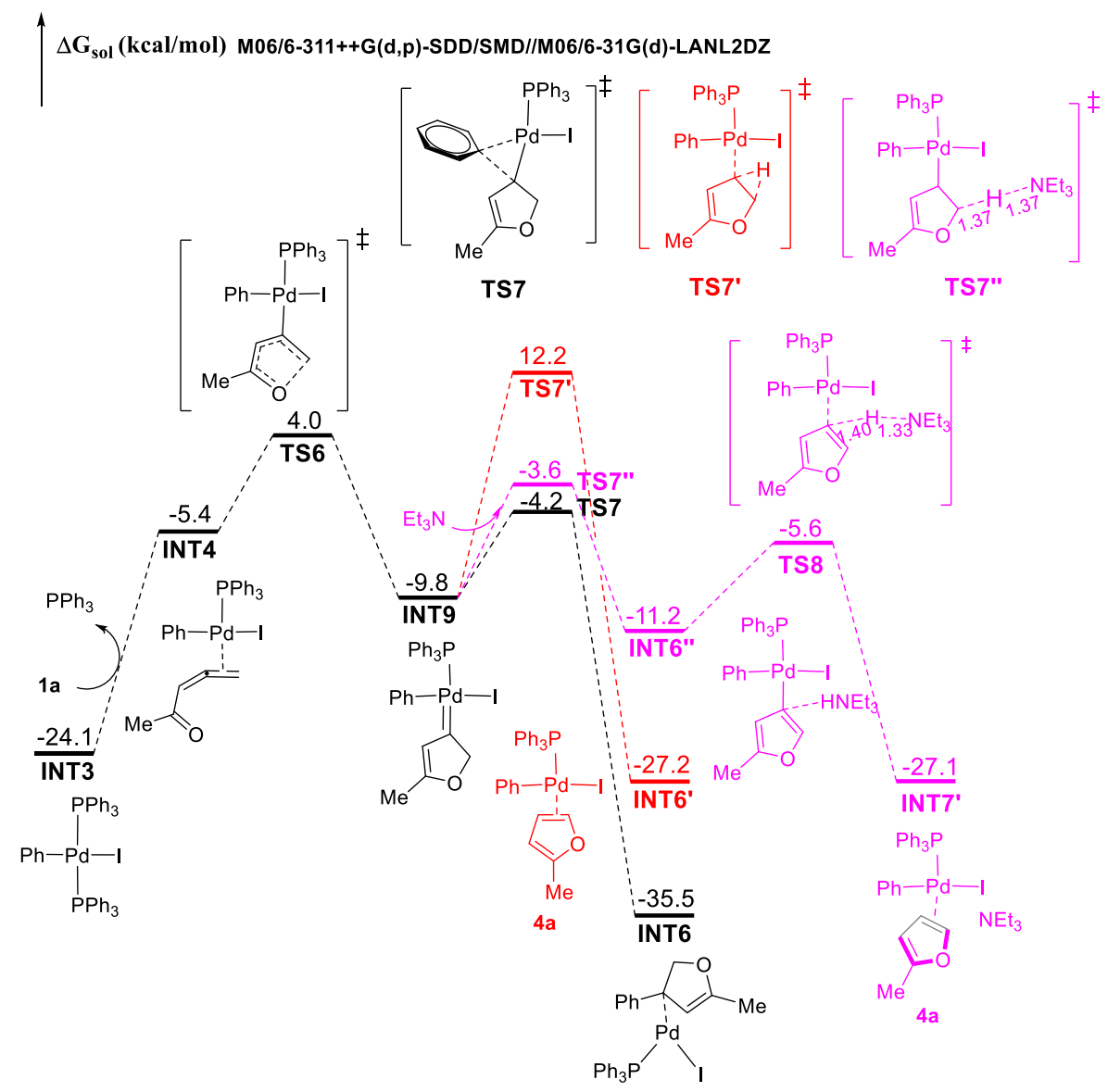

Figure S13. Energy profiles for the formation of the side product $4 \mathrm{a}$ w/o the base $\mathrm{Et}_{3} \mathrm{~N}$. 


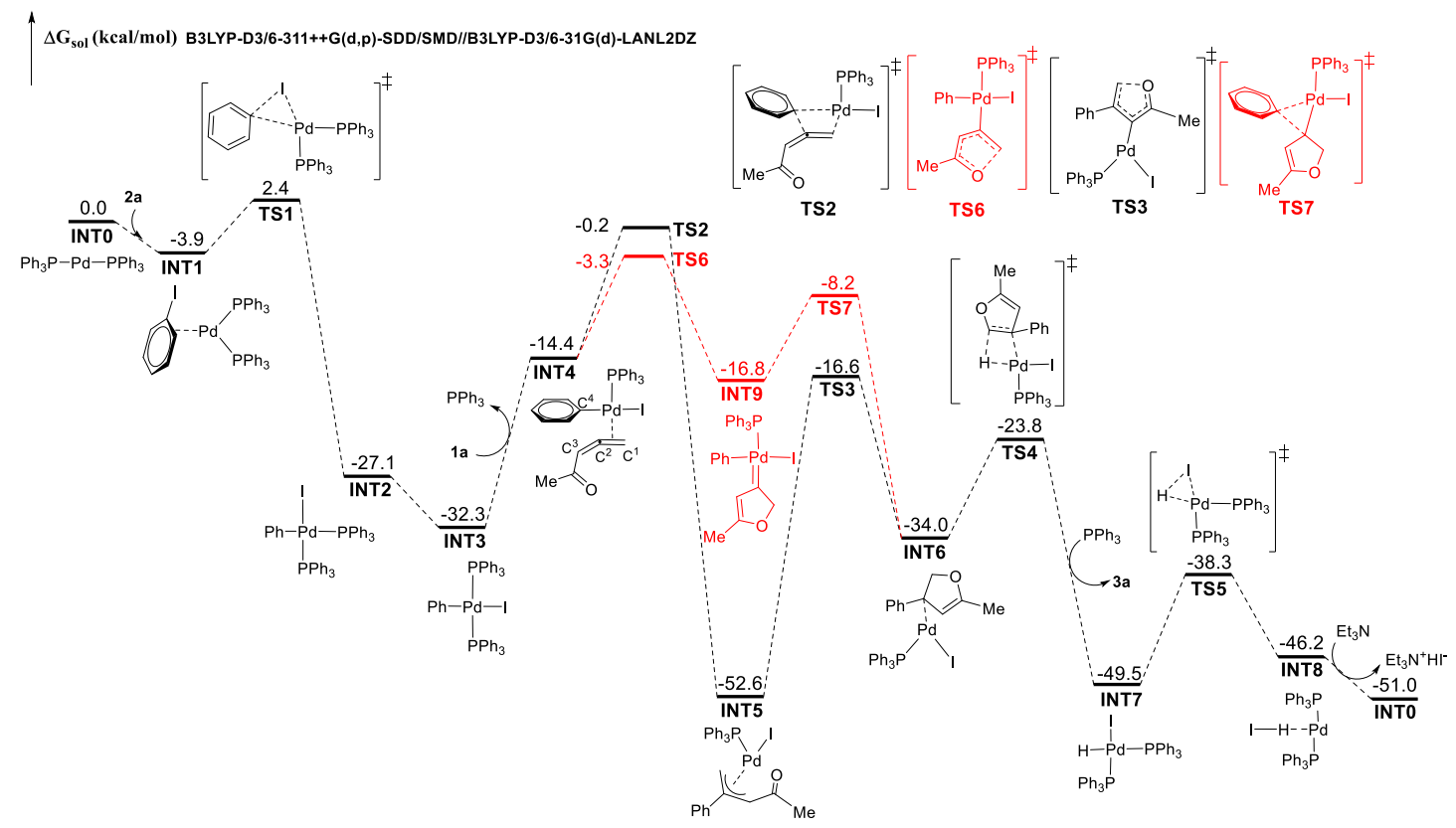

Figure S14. Energy profiles for the $\mathrm{Pd}(0)$-catalyzed coupling cyclization of 1a with 2a to generate polysubstituted furan 3a at the B3LYP-D3/6-311++G(d,p)-SDD/SMD//B3LYP-D3/6-31G(d)-LANL2DZ level of theory.

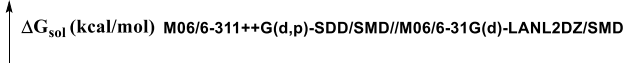
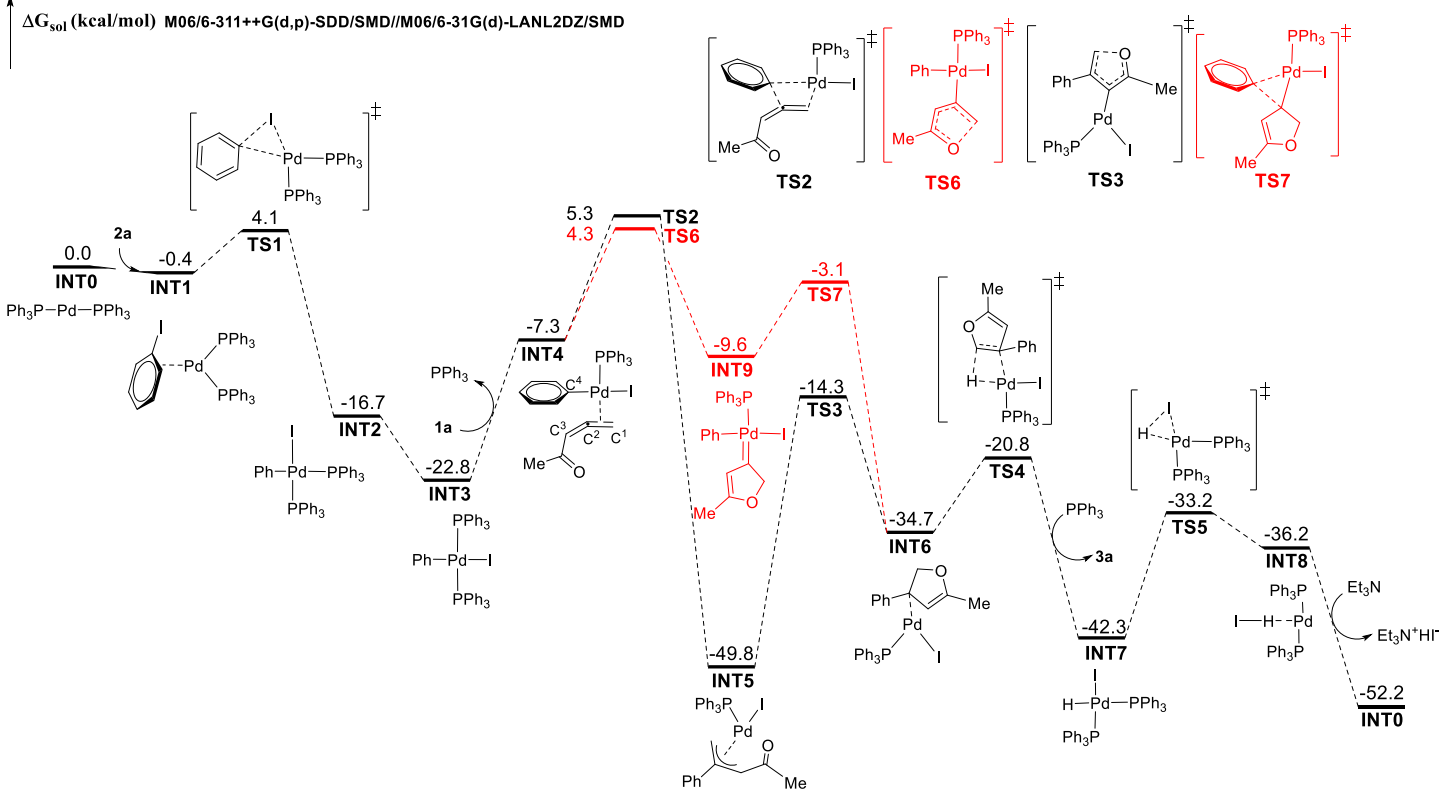

Figure S15. Energy profiles for the $\mathrm{Pd}(0)$-catalyzed coupling cyclization of 1a with $\mathbf{2 a}$ to generate polysubstituted furan 3a at the M06/6-311++G(d,p)-SDD/SMD//M06/6-31G(d)-LANL2DZ/SMD level of theory. The geometry optimizations were carried out with SMD solvation method. 

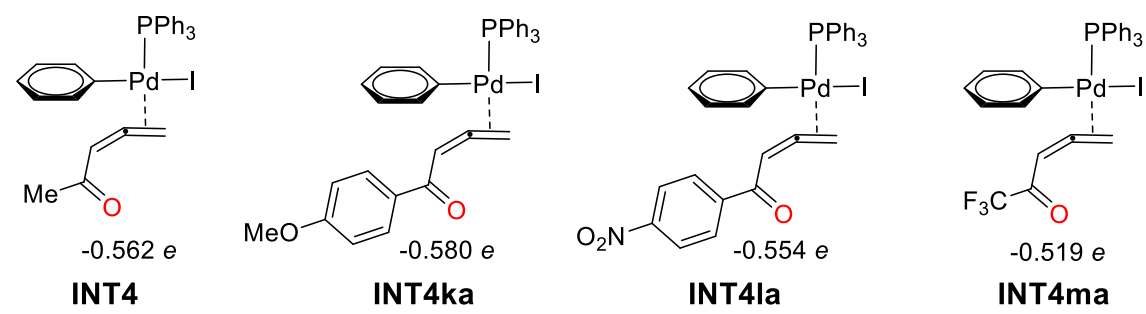

Figure S16. The NBO charge of O atom in INT4, and INT4ka INT4ma. 


\section{Cartesian Coordinates and Energies}

1a

\begin{tabular}{|c|c|c|c|c|c|}
\hline \multirow{2}{*}{$\begin{array}{l}\text { Center } \\
\text { Number }\end{array}$} & \multirow{2}{*}{$\begin{array}{l}\text { Atomic } \\
\text { Number }\end{array}$} & \multirow{2}{*}{$\begin{array}{l}\text { Atomic } \\
\text { Type }\end{array}$} & \multicolumn{3}{|c|}{ Coordinates (Angstroms) } \\
\hline & & & $\mathrm{X}$ & $\mathrm{Y}$ & $\mathrm{Z}$ \\
\hline 1 & 6 & 0 & 0.235601 & -0.766154 & -0.000472 \\
\hline 2 & 1 & 0 & 0.018226 & -1.836631 & -0.001025 \\
\hline 3 & 6 & 0 & 1.480399 & -0.349679 & -0.000095 \\
\hline 4 & 6 & 0 & 2.706849 & 0.077556 & 0.000330 \\
\hline 5 & 1 & 0 & 3.240035 & 0.270961 & 0.930260 \\
\hline 6 & 6 & 0 & -0.911322 & 0.185831 & -0.000139 \\
\hline 7 & 8 & 0 & -0.764477 & 1.389868 & -0.000170 \\
\hline 8 & 6 & 0 & -2.271220 & -0.465102 & 0.000353 \\
\hline 9 & 1 & 0 & -2.386487 & -1.109671 & 0.882574 \\
\hline 10 & 1 & 0 & -2.386725 & -1.110893 & -0.880933 \\
\hline 11 & 1 & 0 & 3.240153 & 0.271901 & -0.929360 \\
\hline 12 & 1 & 0 & -3.051235 & 0.300675 & -0.000023 \\
\hline \multicolumn{6}{|c|}{0.093190 (Hartree/Particle) } \\
\hline \multicolumn{4}{|c|}{ Thermal correction to Energy= } & \multicolumn{2}{|c|}{0.100209} \\
\hline \multicolumn{4}{|c|}{ Thermal correction to Enthalpy= } & \multicolumn{2}{|c|}{0.101154} \\
\hline \multicolumn{4}{|c|}{ Thermal correction to Gibbs Free Energy= } & \multicolumn{2}{|c|}{.061662} \\
\hline \multirow{2}{*}{\multicolumn{4}{|c|}{ Sum of electronic and zero-point Energies $=$}} & \multicolumn{2}{|l|}{-269.019282} \\
\hline & & \multicolumn{2}{|c|}{ Sum of electronic and thermal Energies $=$} & \multicolumn{2}{|l|}{-269.012262} \\
\hline \multicolumn{4}{|c|}{ Sum of electronic and thermal Enthalpies $=$} & \multicolumn{2}{|l|}{-269.011318} \\
\hline \multicolumn{4}{|c|}{ Sum of electronic and thermal Free Energies= } & \multicolumn{2}{|l|}{-269.050810} \\
\hline
\end{tabular}

2a

\begin{tabular}{|c|c|c|c|c|c|}
\hline \multirow{2}{*}{$\begin{array}{l}\text { Center } \\
\text { Number }\end{array}$} & \multirow{2}{*}{$\begin{array}{l}\text { Atomic } \\
\text { Number }\end{array}$} & \multirow{2}{*}{$\begin{array}{l}\text { Atomic } \\
\text { Type }\end{array}$} & \multicolumn{3}{|c|}{ Coordinates (Angstroms) } \\
\hline & & & $\mathrm{X}$ & $\mathrm{Y}$ & Z \\
\hline 1 & 6 & 0 & -3.344678 & 0.000000 & 0.000017 \\
\hline 2 & 6 & 0 & -2.647329 & -1.203642 & 0.000018 \\
\hline 3 & 6 & 0 & -1.255347 & -1.211709 & 0.000012 \\
\hline 4 & 6 & 0 & -0.573969 & 0.000003 & 0.000004 \\
\hline 5 & 6 & 0 & -1.255349 & 1.211710 & 0.000003 \\
\hline 6 & 6 & 0 & -2.647333 & 1.203639 & 0.000009 \\
\hline 7 & 1 & 0 & -3.187105 & -2.149309 & 0.000024 \\
\hline 8 & 1 & 0 & -0.709187 & -2.152550 & 0.000012 \\
\hline 9 & 1 & 0 & -0.709195 & 2.152555 & -0.000003 \\
\hline 10 & 1 & 0 & -3.187108 & 2.149307 & 0.000008 \\
\hline 11 & 53 & 0 & 1.557917 & 0.000000 & -0.000008 \\
\hline 12 & 1 & 0 & -4.432975 & -0.000005 & 0.000022 \\
\hline \multicolumn{6}{|c|}{0.089681 (Hartree/Particle) } \\
\hline \multicolumn{4}{|c|}{ Thermal correction to Energy= } & \multicolumn{2}{|c|}{$\begin{array}{l}0.089681 \text { (Hartree/Particle) } \\
0.095611\end{array}$} \\
\hline \multicolumn{4}{|c|}{ Thermal correction to Enthalpy= } & \multicolumn{2}{|c|}{0.096556} \\
\hline \multicolumn{4}{|c|}{ Thermal correction to Gibbs Free Energy= } & \multicolumn{2}{|c|}{.057904} \\
\hline \multicolumn{4}{|c|}{ Sum of electronic and zero-point Energies $=$} & \multicolumn{2}{|l|}{-242.713837} \\
\hline \multicolumn{4}{|c|}{ Sum of electronic and thermal Energies $=$} & \multicolumn{2}{|l|}{-242.707906} \\
\hline \multicolumn{4}{|c|}{ Sum of electronic and thermal Enthalpies $=$} & \multicolumn{2}{|l|}{-242.706962} \\
\hline \multirow{2}{*}{\multicolumn{4}{|c|}{$\begin{array}{l}\text { Sum of electronic and thermal Free Energies= } \\
\text { M06 /6-311++G(d,p)-SDD/SMD//M06/6-31G }\end{array}$}} & \multicolumn{2}{|l|}{-242.745614} \\
\hline & & & & 2DZ energy in & \\
\hline
\end{tabular}

$\mathbf{P P h}_{3}$

\begin{tabular}{|c|c|c|c|c|c|}
\hline \multirow{2}{*}{$\begin{array}{l}\text { Center } \\
\text { Number }\end{array}$} & \multirow{2}{*}{$\begin{array}{l}\text { Atomic } \\
\text { Number }\end{array}$} & \multirow{2}{*}{$\begin{array}{l}\text { Atomic } \\
\text { Type }\end{array}$} & \multicolumn{3}{|c|}{ Coordinates (Angstroms) } \\
\hline & & & $\mathrm{X}$ & $\mathrm{Y}$ & \\
\hline 1 & 15 & 0 & 0.004196 & -0.003404 & -1.276746 \\
\hline 2 & 6 & 0 & 1.597961 & -0.403344 & -0.441859 \\
\hline 3 & 6 & 0 & 2.348386 & -1.448372 & -0.991689 \\
\hline 4 & 6 & 0 & 2.085318 & 0.252170 & 0.691705 \\
\hline 5 & 6 & 0 & 3.550481 & -1.840682 & -0.416165 \\
\hline 6 & 1 & 0 & 1.980152 & -1.959595 & -1.882916 \\
\hline 7 & 6 & 0 & 3.295691 & -0.131984 & 1.260898 \\
\hline 8 & 1 & 0 & 1.512744 & 1.068526 & 1.132384 \\
\hline 9 & 6 & 0 & 4.028323 & -1.178933 & 0.711258 \\
\hline 10 & 1 & 0 & 4.120712 & -2.659173 & -0.852679 \\
\hline 11 & 1 & 0 & 3.666591 & 0.388417 & 2.142883 \\
\hline 12 & 1 & 0 & 4.974387 & -1.477888 & 1.159928 \\
\hline 13 & 6 & 0 & -0.445808 & 1.579422 & -0.446296 \\
\hline 14 & 6 & 0 & 0.122426 & 2.747198 & -0.968648 \\
\hline 15 & 6 & 0 & -1.300090 & 1.680885 & 0.653803 \\
\hline 16 & 6 & 0 & -0.142652 & 3.985078 & -0.396647 \\
\hline 17 & 1 & 0 & 0.783086 & 2.678895 & -1.834805 \\
\hline 18 & 6 & 0 & -1.576725 & 2.922795 & 1.218690 \\
\hline 19 & 1 & 0 & -1.751414 & 0.782628 & 1.075209 \\
\hline 20 & 6 & 0 & -0.997602 & 4.075023 & 0.698302 \\
\hline 21 & 1 & 0 & 0.311400 & 4.883601 & -0.811636 \\
\hline 22 & 1 & 0 & -2.245709 & 2.988923 & 2.075477 \\
\hline 23 & 1 & 0 & -1.214651 & 5.044978 & 1.142787 \\
\hline 24 & 6 & 0 & -1.143204 & -1.182853 & -0.447447 \\
\hline 25 & 6 & 0 & -2.424666 & -1.301787 & -0.996938 \\
\hline
\end{tabular}




\begin{tabular}{|c|c|c|c|c|c|}
\hline 26 & 6 & 0 & -0.824094 & -1.936087 & 0.684805 \\
\hline 27 & 6 & 0 & -3.371165 & -2.139908 & -0.420293 \\
\hline 28 & 1 & 0 & -2.680546 & -0.725455 & -1.887837 \\
\hline 29 & 6 & 0 & -1.767488 & -2.786050 & 1.254762 \\
\hline 30 & 1 & 0 & 0.169375 & -1.856329 & 1.125944 \\
\hline 31 & 6 & 0 & -3.041852 & -2.887025 & 0.707018 \\
\hline 32 & 1 & 0 & -4.365584 & -2.218134 & -0.857020 \\
\hline 33 & 1 & 0 & -1.504908 & -3.369904 & 2.135824 \\
\hline 34 & 1 & 0 & -3.778021 & -3.551706 & 1.156105 \\
\hline \multicolumn{4}{|c|}{ Zero-point correction= } & \multicolumn{2}{|c|}{0.272975 (Hartree/Particle) } \\
\hline \multicolumn{4}{|c|}{ Thermal correction to Energy= } & \multicolumn{2}{|c|}{0.288910} \\
\hline \multirow{2}{*}{\multicolumn{4}{|c|}{ Thermal correction to Enthalpy= }} & \multicolumn{2}{|c|}{0.289854} \\
\hline & & & & \multicolumn{2}{|c|}{226557} \\
\hline \multicolumn{4}{|c|}{$\begin{array}{l}\text { Thermal correction to Gibbs Free Energy= } \\
\text { Sum of electronic and zero-point Energies= }\end{array}$} & \multirow{2}{*}{\multicolumn{2}{|c|}{$\begin{array}{c}-1035.443649 \\
-1035.427713\end{array}$}} \\
\hline \multicolumn{4}{|c|}{ Sum of electronic and thermal Energies $=$} & & \\
\hline \multicolumn{4}{|c|}{ Sum of electronic and thermal Enthalpies= } & \multicolumn{2}{|c|}{-1035.426769} \\
\hline \multicolumn{4}{|c|}{ Sum of electronic and thermal Free Energies= } & \multicolumn{2}{|l|}{-1035.490067} \\
\hline
\end{tabular}

\section{INTO}

\begin{tabular}{|c|c|c|c|c|c|}
\hline \multirow{2}{*}{$\begin{array}{l}\text { Center } \\
\text { Number }\end{array}$} & \multirow{2}{*}{$\begin{array}{l}\text { Atomic } \\
\text { Number }\end{array}$} & \multirow{2}{*}{$\begin{array}{l}\text { Atomic } \\
\text { Type }\end{array}$} & \multicolumn{3}{|c|}{ Coordinates (Angstroms) } \\
\hline & & & $\mathrm{X}$ & $\mathrm{Y}$ & Z \\
\hline 1 & 46 & 0 & -0.002161 & 0.076638 & 0.533597 \\
\hline 2 & 15 & 0 & -2.300068 & 0.018141 & 0.197463 \\
\hline 3 & 15 & 0 & 2.294406 & 0.032494 & 0.190524 \\
\hline 4 & 6 & 0 & 2.959273 & 1.559181 & -0.582425 \\
\hline 5 & 6 & 0 & 2.432859 & 2.777656 & -0.140262 \\
\hline 6 & 6 & 0 & 3.959695 & 1.563573 & -1.557948 \\
\hline 7 & 6 & 0 & 2.912706 & 3.979527 & -0.647387 \\
\hline 8 & 1 & 0 & 1.631616 & 2.770412 & 0.601634 \\
\hline 9 & 6 & 0 & 4.430225 & 2.767530 & -2.074850 \\
\hline 10 & 1 & 0 & 4.371321 & 0.620131 & -1.918249 \\
\hline 11 & 6 & 0 & 3.911633 & 3.975181 & -1.617565 \\
\hline 12 & 1 & 0 & 2.498995 & 4.921904 & -0.291433 \\
\hline 13 & 1 & 0 & 5.206342 & 2.760976 & -2.838872 \\
\hline 14 & 1 & 0 & 4.282197 & 4.915771 & -2.022638 \\
\hline 15 & 6 & 0 & 2.786201 & -1.284967 & -0.992620 \\
\hline 16 & 6 & 0 & 4.007646 & -1.961425 & -0.936267 \\
\hline 17 & 6 & 0 & 1.872818 & -1.611890 & -2.000892 \\
\hline 18 & 6 & 0 & 4.308056 & -2.945645 & -1.874122 \\
\hline 19 & 1 & 0 & 4.725680 & -1.723306 & -0.150638 \\
\hline 20 & 6 & 0 & 2.177020 & -2.589126 & -2.941074 \\
\hline 21 & 1 & 0 & 0.910267 & -1.095709 & -2.029856 \\
\hline 22 & 6 & 0 & 3.395750 & -3.259930 & -2.876788 \\
\hline 23 & 1 & 0 & 5.260179 & -3.471635 & -1.818441 \\
\hline 24 & 1 & 0 & 1.455449 & -2.832229 & -3.719841 \\
\hline 25 & 1 & 0 & 3.632318 & -4.033405 & -3.606051 \\
\hline 26 & 6 & 0 & 3.443220 & -0.242218 & 1.596226 \\
\hline 27 & 6 & 0 & 3.045610 & -1.161042 & 2.573459 \\
\hline 28 & 6 & 0 & 4.677719 & 0.398752 & 1.726541 \\
\hline 29 & 6 & 0 & 3.874832 & -1.447906 & 3.650890 \\
\hline 30 & 1 & 0 & 2.070495 & -1.643714 & 2.482478 \\
\hline 31 & 6 & 0 & 5.503198 & 0.117829 & 2.811802 \\
\hline 32 & 1 & 0 & 4.995500 & 1.123165 & 0.975646 \\
\hline 33 & 6 & 0 & 5.105278 & -0.806702 & 3.772195 \\
\hline 34 & 1 & 0 & 3.555392 & -2.166118 & 4.404525 \\
\hline 35 & 1 & 0 & 6.462547 & 0.624656 & 2.906990 \\
\hline 36 & 1 & 0 & 5.751899 & -1.024029 & 4.621038 \\
\hline 37 & 6 & 0 & -3.066780 & 1.625079 & -0.252750 \\
\hline 38 & 6 & 0 & -2.274402 & 2.536653 & -0.958881 \\
\hline 39 & 6 & 0 & -4.385702 & 1.967204 & 0.057539 \\
\hline 40 & 6 & 0 & -2.797524 & 3.758564 & -1.365838 \\
\hline 41 & 1 & 0 & -1.235046 & 2.277242 & -1.175411 \\
\hline 42 & 6 & 0 & -4.905001 & 3.195459 & -0.341836 \\
\hline 43 & 1 & 0 & -5.010619 & 1.271080 & 0.617991 \\
\hline 44 & 6 & 0 & -4.114128 & 4.089734 & -1.056648 \\
\hline 45 & 1 & 0 & -2.172529 & 4.458600 & -1.918299 \\
\hline 46 & 1 & 0 & -5.933002 & 3.454612 & -0.092133 \\
\hline 47 & 1 & 0 & -4.522091 & 5.050193 & -1.368305 \\
\hline 48 & 6 & 0 & -3.407313 & -0.597685 & 1.526407 \\
\hline 49 & 6 & 0 & -4.529440 & -1.397639 & 1.296440 \\
\hline 50 & 6 & 0 & -3.098206 & -0.216523 & 2.836444 \\
\hline 51 & 6 & 0 & -5.333085 & -1.800261 & 2.359402 \\
\hline 52 & 1 & 0 & -4.775479 & -1.707320 & 0.280328 \\
\hline 53 & 6 & 0 & -3.907203 & -0.610036 & 3.895598 \\
\hline 54 & 1 & 0 & -2.206825 & 0.386975 & 3.018257 \\
\hline 55 & 6 & 0 & -5.025610 & -1.405038 & 3.657628 \\
\hline 56 & 1 & 0 & -6.203556 & -2.426978 & 2.170620 \\
\hline 57 & 1 & 0 & -3.658472 & -0.305844 & 4.911184 \\
\hline 58 & 1 & 0 & -5.655057 & -1.722592 & 4.487652 \\
\hline 59 & 6 & 0 & -2.724800 & -1.056243 & -1.232481 \\
\hline 60 & 6 & 0 & -1.956093 & -2.211391 & -1.414453 \\
\hline 61 & 6 & 0 & -3.758474 & -0.781050 & -2.131530 \\
\hline 62 & 6 & 0 & -2.222393 & -3.080334 & -2.466281 \\
\hline 63 & 1 & 0 & -1.131136 & -2.412023 & -0.727125 \\
\hline 64 & 6 & 0 & -4.020022 & -1.648265 & -3.188813 \\
\hline 65 & 1 & 0 & -4.359540 & 0.120673 & -2.010184 \\
\hline 66 & 6 & 0 & -3.254676 & -2.797818 & -3.356717 \\
\hline 67 & 1 & 0 & -1.615792 & -3.975567 & -2.595148 \\
\hline 68 & 1 & 0 & -4.824779 & -1.421729 & -3.886642 \\
\hline
\end{tabular}




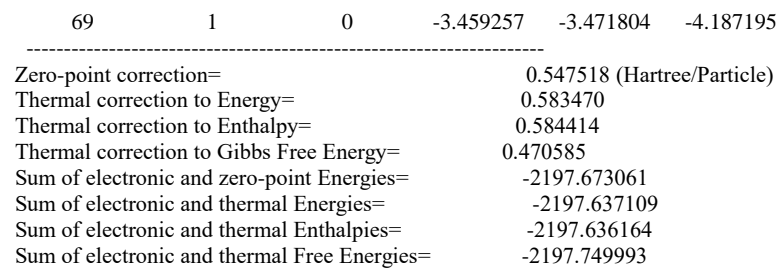

M06 /6-311++G(d,p)-SDD/SMD//M06/6-31G(d)-LANL2DZ energy in toluene solvent $=-2199.8312772$

INT

\begin{tabular}{|c|c|c|c|c|c|}
\hline \multirow{2}{*}{$\begin{array}{l}\text { Center } \\
\text { Number }\end{array}$} & \multirow{2}{*}{$\begin{array}{l}\text { Atomic } \\
\text { Number }\end{array}$} & \multirow{2}{*}{$\begin{array}{l}\text { Atomic } \\
\text { Type }\end{array}$} & \multicolumn{3}{|c|}{ Coordinates (Angstroms) } \\
\hline & & & $\mathrm{X}$ & $\mathrm{Y}$ & \\
\hline 1 & 6 & 0 & 1.272140 & -2.602162 & -3.370927 \\
\hline 2 & 6 & 0 & 2.140838 & -2.889134 & -2.315657 \\
\hline 3 & 6 & 0 & 1.646984 & -3.043683 & -1.027242 \\
\hline 4 & 6 & 0 & 0.269958 & -2.902971 & -0.799274 \\
\hline 5 & 6 & 0 & -0.614771 & -2.627616 & -1.851527 \\
\hline 6 & 6 & 0 & -0.089272 & -2.464388 & -3.140805 \\
\hline 7 & 1 & 0 & 3.213401 & -2.965998 & -2.489550 \\
\hline 8 & 1 & 0 & 2.318283 & -3.258292 & -0.196287 \\
\hline 9 & 1 & 0 & -1.689163 & -2.608086 & -1.676639 \\
\hline 10 & 1 & 0 & -0.768602 & -2.245487 & -3.963671 \\
\hline 11 & 53 & 0 & -0.553859 & -3.480115 & 1.099237 \\
\hline 12 & 15 & 0 & 2.043601 & 0.568036 & 0.070543 \\
\hline 13 & 46 & 0 & 0.019401 & -0.556162 & -0.386779 \\
\hline 14 & 15 & 0 & -2.069896 & 0.541671 & -0.165011 \\
\hline 15 & 6 & 0 & 3.419143 & 0.188212 & -1.087658 \\
\hline 16 & 6 & 0 & 3.138020 & 0.235595 & -2.460046 \\
\hline 17 & 6 & 0 & 4.674810 & -0.265604 & -0.677160 \\
\hline 18 & 6 & 0 & 4.103605 & -0.111859 & -3.396696 \\
\hline 19 & 1 & 0 & 2.140582 & 0.535851 & -2.791964 \\
\hline 20 & 6 & 0 & 5.632861 & -0.642737 & -1.615939 \\
\hline 21 & 1 & 0 & 4.909215 & -0.327009 & 0.385181 \\
\hline 22 & 6 & 0 & 5.355305 & -0.556827 & -2.975988 \\
\hline 23 & 1 & 0 & 3.870347 & -0.057000 & -4.459523 \\
\hline 24 & 1 & 0 & 6.605484 & -0.998461 & -1.278320 \\
\hline 25 & 1 & 0 & 6.108509 & -0.844389 & -3.707990 \\
\hline 26 & 6 & 0 & -2.448589 & 1.926700 & -1.311280 \\
\hline 27 & 6 & 0 & -3.749301 & 2.319589 & -1.643631 \\
\hline 28 & 6 & 0 & -1.368385 & 2.649758 & -1.828323 \\
\hline 29 & 6 & 0 & -3.963949 & 3.412788 & -2.477331 \\
\hline 30 & 1 & 0 & -4.599963 & 1.766360 & -1.244045 \\
\hline 31 & 6 & 0 & -1.584625 & 3.751359 & -2.649595 \\
\hline 32 & 1 & 0 & -0.348865 & 2.346646 & -1.578175 \\
\hline 33 & 6 & 0 & -2.882319 & 4.131773 & -2.978843 \\
\hline 34 & 1 & 0 & -4.981281 & 3.706155 & -2.733152 \\
\hline 35 & 1 & 0 & -0.731682 & 4.307688 & -3.035630 \\
\hline 36 & 1 & 0 & -3.052617 & 4.988359 & -3.629567 \\
\hline 37 & 6 & 0 & 2.815067 & 0.367290 & 1.726014 \\
\hline 38 & 6 & 0 & 3.736071 & 1.279680 & 2.252470 \\
\hline 39 & 6 & 0 & 2.464216 & -0.754668 & 2.479722 \\
\hline 40 & 6 & 0 & 4.296417 & 1.068236 & 3.506898 \\
\hline 41 & 1 & 0 & 4.012005 & 2.162518 & 1.673637 \\
\hline 42 & 6 & 0 & 3.027163 & -0.968923 & 3.734833 \\
\hline 43 & 1 & 0 & 1.732606 & -1.456918 & 2.073665 \\
\hline 44 & 6 & 0 & 3.942363 & -0.057003 & 4.249251 \\
\hline 45 & 1 & 0 & 5.011200 & 1.784749 & 3.909392 \\
\hline 46 & 1 & 0 & 2.744220 & -1.847232 & 4.313293 \\
\hline 47 & 1 & 0 & 4.379812 & -0.218338 & 5.233579 \\
\hline 48 & 6 & 0 & 1.878726 & 2.401113 & -0.032347 \\
\hline 49 & 6 & 0 & 1.011345 & 3.011172 & 0.885689 \\
\hline 50 & 6 & 0 & 2.467085 & 3.187696 & -1.024643 \\
\hline 51 & 6 & 0 & 0.737548 & 4.369617 & 0.808080 \\
\hline 52 & 1 & 0 & 0.532878 & 2.410888 & 1.663100 \\
\hline 53 & 6 & 0 & 2.180277 & 4.549774 & -1.109440 \\
\hline 54 & 1 & 0 & 3.155347 & 2.738872 & -1.740376 \\
\hline 55 & 6 & 0 & 1.315197 & 5.143149 & -0.197584 \\
\hline 56 & 1 & 0 & 0.056875 & 4.821547 & 1.528428 \\
\hline 57 & 1 & 0 & 2.645580 & 5.148559 & -1.891642 \\
\hline 58 & 1 & 0 & 1.090487 & 6.206389 & -0.267594 \\
\hline 59 & 6 & 0 & -3.611952 & -0.455993 & -0.206553 \\
\hline 60 & 6 & 0 & -4.370602 & -0.744841 & 0.929306 \\
\hline 61 & 6 & 0 & -3.956142 & -1.069142 & -1.420243 \\
\hline 62 & 6 & 0 & -5.444996 & -1.628588 & 0.855064 \\
\hline 63 & 1 & 0 & -4.118814 & -0.279383 & 1.882263 \\
\hline 64 & 6 & 0 & -5.035257 & -1.939128 & -1.495858 \\
\hline 65 & 1 & 0 & -3.370403 & -0.850582 & -2.316931 \\
\hline 66 & 6 & 0 & -5.780740 & -2.227101 & -0.353596 \\
\hline 67 & 1 & 0 & -6.024953 & -1.846958 & 1.750832 \\
\hline 68 & 1 & 0 & -5.294329 & -2.400102 & -2.448101 \\
\hline 69 & 1 & 0 & -6.620646 & -2.917696 & -0.408525 \\
\hline 70 & 6 & 0 & -2.143027 & 1.367373 & 1.479898 \\
\hline 71 & 6 & 0 & -2.733316 & 2.614558 & 1.696728 \\
\hline 72 & 6 & 0 & -1.501976 & 0.727723 & 2.547768 \\
\hline 73 & 6 & 0 & -2.672015 & 3.213246 & 2.953070 \\
\hline 74 & 1 & 0 & -3.226996 & 3.133285 & 0.874564 \\
\hline 75 & 6 & 0 & -1.451960 & 1.318630 & 3.805053 \\
\hline 76 & 1 & 0 & -1.011169 & -0.232274 & 2.371743 \\
\hline
\end{tabular}




$\begin{array}{rrrrrr}77 & 6 & 0 & -2.030948 & 2.569636 & 4.006879 \\ 78 & 1 & 0 & -3.125680 & 4.191812 & 3.106493 \\ 79 & 1 & 0 & -0.941779 & 0.810874 & 4.622465 \\ 80 & 1 & 0 & -1.977661 & 3.044530 & 4.985497 \\ 81 & 1 & 0 & 1.668329 & -2.480722 & -4.378382 \\ - & 1 & & & \end{array}$

Zero-point correction $=\quad 0.639459($ Hartree/Particle $)$

Thermal correction to Energy=

0.682575

Thermal correction to Enthalpy=

Thermal correction to Gibbs Free Energy=

Sum of electronic and zero-point Energies=

Sum of electronic and thermal Energies=

0.683519

Sum of electronic and thermal Enthalpies=

0.557999

$-2440.405126$

$-2440.362009$

$-2440.361065$

M06 /6-311++G(d,p)-SDD/SMD//M06/6-31G(d)-LANL2DZ energy in toluene solvent $=-2442.7560796$

INT2

\begin{tabular}{|c|c|c|c|c|c|}
\hline \multirow{2}{*}{$\begin{array}{l}\text { Center } \\
\text { Number }\end{array}$} & \multirow{2}{*}{$\begin{array}{l}\text { Atomic } \\
\text { Number }\end{array}$} & \multirow{2}{*}{$\begin{array}{c}\text { Atomic } \\
\text { Type }\end{array}$} & \multicolumn{3}{|c|}{ Coordinates (Angstroms) } \\
\hline & & & $\mathrm{X}$ & $\mathrm{Y}$ & $z$ \\
\hline 1 & 6 & 0 & -4.277711 & -3.607099 & 0.610005 \\
\hline 2 & 6 & 0 & -3.897664 & -3.241424 & -0.678717 \\
\hline 3 & 6 & 0 & -2.730991 & -2.508715 & -0.887152 \\
\hline 4 & 6 & 0 & -1.938242 & -2.133484 & 0.194880 \\
\hline 5 & 6 & 0 & -2.310278 & -2.511364 & 1.484266 \\
\hline 6 & 6 & 0 & -3.479295 & -3.244889 & 1.690427 \\
\hline 7 & 1 & 0 & -4.514700 & -3.524440 & -1.531804 \\
\hline 8 & 1 & 0 & -2.444735 & -2.227844 & -1.902624 \\
\hline 9 & 1 & 0 & -1.683083 & -2.245190 & 2.338747 \\
\hline 10 & 1 & 0 & -3.759880 & -3.538744 & 2.702238 \\
\hline 11 & 53 & 0 & 1.052654 & -3.343918 & -0.265056 \\
\hline 12 & 15 & 0 & -1.689802 & 0.878584 & -0.107129 \\
\hline 13 & 46 & 0 & -0.256329 & -0.985302 & -0.004891 \\
\hline 14 & 15 & 0 & 1.915963 & 0.211498 & 0.083496 \\
\hline 15 & 6 & 0 & 2.047452 & 2.047468 & -0.061944 \\
\hline 16 & 6 & 0 & 1.603408 & 2.832495 & 1.010110 \\
\hline 17 & 6 & 0 & 2.505505 & 2.689593 & -1.214358 \\
\hline 18 & 6 & 0 & 1.609433 & 4.219099 & 0.931039 \\
\hline 19 & 1 & 0 & 1.259134 & 2.355570 & 1.926910 \\
\hline 20 & 6 & 0 & 2.519663 & 4.080088 & -1.290225 \\
\hline 21 & 1 & 0 & 2.855504 & 2.103748 & -2.063542 \\
\hline 22 & 6 & 0 & 2.070652 & 4.849059 & -0.222429 \\
\hline 23 & 1 & 0 & 1.257263 & 4.805584 & 1.778979 \\
\hline 24 & 1 & 0 & 2.883481 & 4.561884 & -2.197137 \\
\hline 25 & 1 & 0 & 2.084291 & 5.936274 & -0.286188 \\
\hline 26 & 6 & 0 & 3.103252 & -0.391073 & -1.171245 \\
\hline 27 & 6 & 0 & 4.374631 & -0.873369 & -0.863933 \\
\hline 28 & 6 & 0 & 2.663467 & -0.420923 & -2.499228 \\
\hline 29 & 6 & 0 & 5.199076 & -1.362851 & -1.872966 \\
\hline 30 & 1 & 0 & 4.717622 & -0.892815 & 0.169356 \\
\hline 31 & 6 & 0 & 3.493185 & -0.894585 & -3.506613 \\
\hline 32 & 1 & 0 & 1.653939 & -0.081038 & -2.739981 \\
\hline 33 & 6 & 0 & 4.763954 & -1.369505 & -3.193152 \\
\hline 34 & 1 & 0 & 6.185258 & -1.749569 & -1.621257 \\
\hline 35 & 1 & 0 & 3.140179 & -0.913306 & -4.536346 \\
\hline 36 & 1 & 0 & 5.410584 & -1.757505 & -3.978487 \\
\hline 37 & 6 & 0 & 2.715204 & -0.055626 & 1.714027 \\
\hline 38 & 6 & 0 & 2.150986 & -0.962896 & 2.614662 \\
\hline 39 & 6 & 0 & 3.859201 & 0.657804 & 2.094618 \\
\hline 40 & 6 & 0 & 2.719822 & -1.156953 & 3.870406 \\
\hline 41 & 1 & 0 & 1.266736 & -1.532298 & 2.320104 \\
\hline 42 & 6 & 0 & 4.430503 & 0.457382 & 3.345123 \\
\hline 43 & 1 & 0 & 4.298019 & 1.382302 & 1.407420 \\
\hline 44 & 6 & 0 & 3.859875 & -0.449973 & 4.235270 \\
\hline 45 & 1 & 0 & 2.271989 & -1.869465 & 4.560956 \\
\hline 46 & 1 & 0 & 5.321646 & 1.015341 & 3.628521 \\
\hline 47 & 1 & 0 & 4.306681 & -0.602985 & 5.216615 \\
\hline 48 & 6 & 0 & -1.767151 & 1.874644 & 1.422656 \\
\hline 49 & 6 & 0 & -2.539549 & 3.038428 & 1.519789 \\
\hline 50 & 6 & 0 & -1.067224 & 1.425994 & 2.544517 \\
\hline 51 & 6 & 0 & -2.550884 & 3.774010 & 2.698488 \\
\hline 52 & 1 & 0 & -3.144169 & 3.361052 & 0.671435 \\
\hline 53 & 6 & 0 & -1.088663 & 2.156168 & 3.729705 \\
\hline 54 & 1 & 0 & -0.495326 & 0.497451 & 2.478260 \\
\hline 55 & 6 & 0 & -1.817021 & 3.338748 & 3.800757 \\
\hline 56 & 1 & 0 & -3.143249 & 4.685263 & 2.761957 \\
\hline 57 & 1 & 0 & -0.533314 & 1.799108 & 4.595407 \\
\hline 58 & 1 & 0 & -1.829867 & 3.916445 & 4.723600 \\
\hline 59 & 6 & 0 & -1.131620 & 1.959107 & -1.476350 \\
\hline 60 & 6 & 0 & -0.814814 & 1.305689 & -2.675556 \\
\hline 61 & 6 & 0 & -0.999672 & 3.345317 & -1.403810 \\
\hline 62 & 6 & 0 & -0.406089 & 2.030016 & -3.788986 \\
\hline 63 & 1 & 0 & -0.889879 & 0.215877 & -2.728879 \\
\hline 64 & 6 & 0 & -0.575360 & 4.066729 & -2.516492 \\
\hline 65 & 1 & 0 & -1.193569 & 3.867415 & -0.467880 \\
\hline 66 & 6 & 0 & -0.288777 & 3.415806 & -3.710900 \\
\hline 67 & 1 & 0 & -0.170810 & 1.509526 & -4.716263 \\
\hline 68 & 1 & 0 & -0.459408 & 5.147040 & -2.440947 \\
\hline 69 & 1 & 0 & 0.038290 & 3.985631 & -4.579130 \\
\hline 70 & 6 & 0 & -3.462518 & 0.603882 & -0.476453 \\
\hline 71 & 6 & 0 & -4.282372 & 0.129084 & 0.553489 \\
\hline 72 & 6 & 0 & -3.989953 & 0.736430 & -1.761968 \\
\hline
\end{tabular}




\begin{tabular}{|c|c|c|c|c|c|}
\hline 73 & 6 & 0 & -5.600400 & -0.220127 & 0.296247 \\
\hline 74 & 1 & 0 & -3.877908 & 0.006873 & 1.558195 \\
\hline 75 & 6 & 0 & -5.313788 & 0.388964 & -2.015269 \\
\hline 76 & 1 & 0 & -3.369472 & 1.111326 & -2.574851 \\
\hline 77 & 6 & 0 & -6.118295 & -0.094917 & -0.990325 \\
\hline 78 & 1 & 0 & -6.221359 & -0.606330 & 1.102858 \\
\hline 79 & 1 & 0 & -5.715943 & 0.498823 & -3.021229 \\
\hline 80 & 1 & 0 & -7.150906 & -0.374666 & -1.192884 \\
\hline 81 & 1 & 0 & -5.190601 & -4.179405 & 0.770833 \\
\hline \multicolumn{4}{|c|}{ Zero-point correction $=$} & \multicolumn{2}{|c|}{0.640924 (Hartree/Particle) } \\
\hline Thermal & a to & & & \multicolumn{2}{|c|}{0.683309} \\
\hline Thermal & $\mathrm{n}$ to & & & \multicolumn{2}{|c|}{0.684253} \\
\hline Thermal & & e Energy $=$ & & \multicolumn{2}{|c|}{0.562505} \\
\hline Sum of e & and & t Energies= & & \multicolumn{2}{|l|}{-2440.428714} \\
\hline Sum of e & and & Energies $=$ & & \multicolumn{2}{|l|}{-2440.386329} \\
\hline Sum of e & and & Enthalpies $=$ & & \multicolumn{2}{|l|}{-2440.385385} \\
\hline Sum of e & $\mathrm{c}$ and & ee Energies & & \multicolumn{2}{|l|}{-2440.507133} \\
\hline
\end{tabular}

作

\section{INT3}

\begin{tabular}{|c|c|c|c|c|c|}
\hline \multirow{2}{*}{$\begin{array}{l}\text { Center } \\
\text { Number }\end{array}$} & \multirow{2}{*}{$\begin{array}{l}\text { Atomic } \\
\text { Number }\end{array}$} & \multirow{2}{*}{$\begin{array}{l}\text { Atomic } \\
\text { Type }\end{array}$} & \multicolumn{3}{|c|}{ Coordinates (Angstroms) } \\
\hline & & & $\mathrm{X}$ & $\mathrm{Y}$ & Z \\
\hline 1 & 46 & 0 & 0.000002 & 0.011074 & -0.000059 \\
\hline 2 & 15 & 0 & -2.375171 & -0.014651 & 0.006783 \\
\hline 3 & 15 & 0 & 2.375201 & -0.014808 & -0.006759 \\
\hline 4 & 6 & 0 & -0.000155 & -2.035843 & 0.000124 \\
\hline 5 & 6 & 0 & -0.312425 & -2.748874 & -1.160033 \\
\hline 6 & 1 & 0 & -0.577131 & -2.218352 & -2.077903 \\
\hline 7 & 6 & 0 & 0.311968 & -2.748692 & 1.160442 \\
\hline 8 & 6 & 0 & -0.316020 & -4.143148 & -1.159688 \\
\hline 9 & 6 & 0 & -0.000429 & -4.845233 & 0.000421 \\
\hline 10 & 6 & 0 & 0.315292 & -4.142968 & 1.160387 \\
\hline 11 & 1 & 0 & 0.573749 & -4.681242 & 2.072696 \\
\hline 12 & 1 & 0 & 0.576774 & -2.218057 & 2.078208 \\
\hline 13 & 1 & 0 & -0.574676 & -4.681547 & -2.071865 \\
\hline 14 & 6 & 0 & -3.202460 & -1.611407 & -0.349673 \\
\hline 15 & 6 & 0 & -3.840234 & -1.872799 & -1.562613 \\
\hline 16 & 6 & 0 & -3.101139 & -2.633069 & 0.601745 \\
\hline 17 & 6 & 0 & -4.359689 & -3.138947 & -1.824141 \\
\hline 18 & 1 & 0 & -3.937067 & -1.087004 & -2.310468 \\
\hline 19 & 6 & 0 & -3.619749 & -3.892563 & 0.339648 \\
\hline 20 & 1 & 0 & -2.588894 & -2.446442 & 1.546204 \\
\hline 21 & 6 & 0 & -4.247226 & -4.149822 & -0.878004 \\
\hline 22 & 1 & 0 & -4.856626 & -3.330204 & -2.774133 \\
\hline 23 & 1 & 0 & -3.518803 & -4.681265 & 1.083473 \\
\hline 24 & 1 & 0 & -4.649230 & -5.140318 & -1.085792 \\
\hline 25 & 6 & 0 & -3.049903 & 1.122563 & -1.253211 \\
\hline 26 & 6 & 0 & -4.063722 & 2.040454 & -0.984032 \\
\hline 27 & 6 & 0 & -2.488651 & 1.075853 & -2.534370 \\
\hline 28 & 6 & 0 & -4.512020 & 2.898987 & -1.984222 \\
\hline 29 & 1 & 0 & -4.491397 & 2.106243 & 0.015641 \\
\hline 30 & 6 & 0 & -2.948006 & 1.922238 & -3.534111 \\
\hline 31 & 1 & 0 & -1.671831 & 0.380157 & -2.740578 \\
\hline 32 & 6 & 0 & -3.959149 & 2.839506 & -3.257911 \\
\hline 33 & 1 & 0 & -5.293864 & 3.623227 & -1.761800 \\
\hline 34 & 1 & 0 & -2.504282 & 1.878317 & -4.527322 \\
\hline 35 & 1 & 0 & -4.309128 & 3.515444 & -4.036387 \\
\hline 36 & 6 & 0 & -3.121987 & 0.467292 & 1.604094 \\
\hline 37 & 6 & 0 & -4.470437 & 0.201389 & 1.874016 \\
\hline 38 & 6 & 0 & -2.342283 & 1.104155 & 2.572600 \\
\hline 39 & 6 & 0 & -5.030360 & 0.579642 & 3.088006 \\
\hline 40 & 1 & 0 & -5.083091 & -0.309101 & 1.130098 \\
\hline 41 & 6 & 0 & -2.906105 & 1.477857 & 3.788995 \\
\hline 42 & 1 & 0 & -1.295683 & 1.325306 & 2.358518 \\
\hline 43 & 6 & 0 & -4.247331 & 1.218063 & 4.047053 \\
\hline 44 & 1 & 0 & -6.080089 & 0.371071 & 3.288394 \\
\hline 45 & 1 & 0 & -2.291693 & 1.977876 & 4.535554 \\
\hline 46 & 1 & 0 & -4.686094 & 1.511751 & 4.999461 \\
\hline 47 & 6 & 0 & 3.049885 & 1.122433 & 1.253245 \\
\hline 48 & 6 & 0 & 4.063351 & 2.040675 & 0.983915 \\
\hline 49 & 6 & 0 & 2.488798 & 1.075592 & 2.534475 \\
\hline 50 & 6 & 0 & 4.511425 & 2.899449 & 1.983999 \\
\hline 51 & 1 & 0 & 4.490914 & 2.106527 & -0.015801 \\
\hline 52 & 6 & 0 & 2.947925 & 1.922228 & 3.534104 \\
\hline 53 & 1 & 0 & 1.672307 & 0.379578 & 2.740877 \\
\hline 54 & 6 & 0 & 3.958676 & 2.839878 & 3.257732 \\
\hline 55 & 1 & 0 & 5.292991 & 3.623952 & 1.761458 \\
\hline 56 & 1 & 0 & 2.504315 & 1.878218 & 4.527362 \\
\hline 57 & 1 & 0 & 4.308461 & 3.516020 & 4.036119 \\
\hline 58 & 6 & 0 & 3.122261 & 0.467025 & -1.603981 \\
\hline 59 & 6 & 0 & 4.470695 & 0.200984 & -1.873826 \\
\hline 60 & 6 & 0 & 2.342656 & 1.103966 & -2.572518 \\
\hline 61 & 6 & 0 & 5.030688 & 0.579155 & -3.087808 \\
\hline 62 & 1 & 0 & 5.083255 & -0.309537 & -1.129851 \\
\hline 63 & 6 & 0 & 2.906550 & 1.477581 & -3.788907 \\
\hline 64 & 1 & 0 & 1.296082 & 1.325237 & -2.358432 \\
\hline 65 & 6 & 0 & 4.247754 & 1.217628 & -4.046903 \\
\hline 66 & 1 & 0 & 6.080401 & 0.370476 & -3.288168 \\
\hline 67 & 1 & 0 & 2.292219 & 1.977647 & -4.535502 \\
\hline 68 & 1 & 0 & 4.686595 & 1.511214 & -4.999308 \\
\hline
\end{tabular}




$\begin{array}{rrrrrc}69 & 6 & 0 & 3.202317 & -1.611635 & 0.349756 \\ 70 & 6 & 0 & 3.840654 & -1.872958 & 1.562402 \\ 71 & 6 & 0 & 3.100486 & -2.633353 & -0.601558 \\ 72 & 6 & 0 & 4.360233 & -3.139101 & 1.823728 \\ 73 & 1 & 0 & 3.937794 & -1.087123 & 2.310179 \\ 74 & 6 & 0 & 3.619216 & -3.892829 & -0.339669 \\ 75 & 1 & 0 & 2.587629 & -2.446756 & -1.545692 \\ 76 & 6 & 0 & 4.247328 & -4.150008 & 0.877676 \\ 77 & 1 & 0 & 4.857571 & -3.330359 & 2.773511 \\ 78 & 1 & 0 & 3.517861 & -4.681586 & -1.083383 \\ 79 & 1 & 0 & 4.649450 & -5.140487 & 1.085318 \\ 80 & 53 & 0 & 0.000172 & 2.801699 & -0.000136 \\ 81 & 1 & 0 & -0.000549 & -5.934517 & 0.000543 \\ ------------------ & & \end{array}$

Zero-point correction= 0.639455 (Hartree/Particle)

Thermal correction to Energy= 0.682444 Thermal correction to Enthalpy

Thermal correction to Gibbs Free Energy=

Sum of electronic and zero-point Energies

Sum of electronic and thermal Energies=

Sum of electronic and thermal Enthalpies=

$$
0.683388
$$$$
0.557493
$$

$-2440.435510$

$-2440.392521$

$-2440.391577$

M06 /6-311++G(d,p)-SDD/SMD//M06/6-31G(d)-LANL2DZ energy in toluene solvent $=-2442.7942632$

INT3'

\begin{tabular}{|c|c|c|c|c|c|}
\hline \multirow{2}{*}{$\begin{array}{l}\text { Center } \\
\text { Number }\end{array}$} & \multirow{2}{*}{$\begin{array}{l}\text { Atomic } \\
\text { Number }\end{array}$} & \multirow{2}{*}{$\begin{array}{l}\text { Atomic } \\
\text { Type }\end{array}$} & \multicolumn{3}{|c|}{ Coordinates (Angstroms) } \\
\hline & & & $\mathrm{X}$ & $\mathrm{Y}$ & \\
\hline 1 & 6 & 0 & -1.094245 & 4.495173 & -1.327334 \\
\hline 2 & 6 & 0 & -0.903176 & 4.212424 & 0.021293 \\
\hline 3 & 6 & 0 & -0.639531 & 2.904782 & 0.434355 \\
\hline 4 & 6 & 0 & -0.561706 & 1.874847 & -0.503968 \\
\hline 5 & 6 & 0 & -0.769875 & 2.154474 & -1.853343 \\
\hline 6 & 6 & 0 & -1.027028 & 3.463392 & -2.259135 \\
\hline 7 & 1 & 0 & -0.961684 & 5.007808 & 0.764167 \\
\hline 8 & 1 & 0 & -0.511302 & 2.703818 & 1.499274 \\
\hline 9 & 1 & 0 & -0.737331 & 1.348720 & -2.588978 \\
\hline 10 & 1 & 0 & -1.190698 & 3.667789 & -3.317261 \\
\hline 11 & 53 & 0 & -0.140974 & -1.443843 & -2.897369 \\
\hline 12 & 15 & 0 & -2.450490 & -0.198461 & 0.324878 \\
\hline 13 & 15 & 0 & 2.244098 & 0.705989 & 0.308010 \\
\hline 14 & 6 & 0 & 2.637479 & 2.449405 & -0.088573 \\
\hline 15 & 6 & 0 & 2.405301 & 2.843504 & -1.410977 \\
\hline 16 & 6 & 0 & 3.182078 & 3.363372 & 0.814303 \\
\hline 17 & 6 & 0 & 2.688160 & 4.141739 & -1.812888 \\
\hline 18 & 1 & 0 & 1.984384 & 2.133301 & -2.125036 \\
\hline 19 & 6 & 0 & 3.457778 & 4.666398 & 0.408581 \\
\hline 20 & 1 & 0 & 3.398791 & 3.062879 & 1.838764 \\
\hline 21 & 6 & 0 & 3.203862 & 5.058749 & -0.900552 \\
\hline 22 & 1 & 0 & 2.489464 & 4.441271 & -2.840549 \\
\hline 23 & 1 & 0 & 3.876880 & 5.375109 & 1.121362 \\
\hline 24 & 1 & 0 & 3.415354 & 6.079818 & -1.214037 \\
\hline 25 & 6 & 0 & 2.547045 & 0.490158 & 2.102116 \\
\hline 26 & 6 & 0 & 1.917500 & 1.343247 & 3.021701 \\
\hline 27 & 6 & 0 & 3.193068 & -0.653857 & 2.582739 \\
\hline 28 & 6 & 0 & 1.954048 & 1.066144 & 4.383321 \\
\hline 29 & 1 & 0 & 1.388673 & 2.229599 & 2.668394 \\
\hline 30 & 6 & 0 & 3.216527 & -0.936598 & 3.945613 \\
\hline 31 & 1 & 0 & 3.663478 & -1.345678 & 1.885905 \\
\hline 32 & 6 & 0 & 2.601139 & -0.077232 & 4.848839 \\
\hline 33 & 1 & 0 & 1.472099 & 1.745849 & 5.084859 \\
\hline 34 & 1 & 0 & 3.706457 & -1.843985 & 4.293418 \\
\hline 35 & 1 & 0 & 2.617804 & -0.300003 & 5.914201 \\
\hline 36 & 6 & 0 & 3.660698 & -0.136624 & -0.500441 \\
\hline 37 & 6 & 0 & 3.458423 & -0.859705 & -1.675381 \\
\hline 38 & 6 & 0 & 4.960854 & 0.028566 & -0.005640 \\
\hline 39 & 6 & 0 & 4.541067 & -1.442844 & -2.330832 \\
\hline 40 & 1 & 0 & 2.451262 & -0.965247 & -2.088503 \\
\hline 41 & 6 & 0 & 6.035682 & -0.562226 & -0.656864 \\
\hline 42 & 1 & 0 & 5.132851 & 0.619114 & 0.895138 \\
\hline 43 & 6 & 0 & 5.825916 & -1.304587 & -1.818099 \\
\hline 44 & 1 & 0 & 4.368323 & -2.002811 & -3.248925 \\
\hline 45 & 1 & 0 & 7.042204 & -0.438010 & -0.260527 \\
\hline 46 & 1 & 0 & 6.670651 & -1.765821 & -2.327792 \\
\hline 47 & 6 & 0 & -3.487608 & 0.453111 & -1.026269 \\
\hline 48 & 6 & 0 & -3.823902 & -0.364013 & -2.108307 \\
\hline 49 & 6 & 0 & -3.828254 & 1.809976 & -1.058718 \\
\hline 50 & 6 & 0 & -4.502166 & 0.166701 & -3.199809 \\
\hline 51 & 1 & 0 & -3.541870 & -1.415075 & -2.112275 \\
\hline 52 & 6 & 0 & -4.513291 & 2.333285 & -2.147728 \\
\hline 53 & 1 & 0 & -3.537048 & 2.469691 & -0.241511 \\
\hline 54 & 6 & 0 & -4.848923 & 1.513253 & -3.220934 \\
\hline 55 & 1 & 0 & -4.751171 & -0.477232 & -4.040928 \\
\hline 56 & 1 & 0 & -4.765686 & 3.392516 & -2.163041 \\
\hline 57 & 1 & 0 & -5.375571 & 1.927159 & -4.079492 \\
\hline 58 & 6 & 0 & -3.040417 & 0.663606 & 1.829572 \\
\hline 59 & 6 & 0 & -2.138163 & 0.908260 & 2.867547 \\
\hline 60 & 6 & 0 & -4.385432 & 1.008708 & 2.006059 \\
\hline 61 & 6 & 0 & -2.565912 & 1.483095 & 4.059712 \\
\hline 62 & 1 & 0 & -1.086038 & 0.652144 & 2.730755 \\
\hline 63 & 6 & 0 & -4.812448 & 1.586567 & 3.196532 \\
\hline 64 & 1 & 0 & -5.103944 & 0.819073 & 1.208360 \\
\hline
\end{tabular}




\begin{tabular}{|c|c|c|c|c|c|}
\hline 65 & 6 & 0 & -3.904280 & 1.823658 & 4.225331 \\
\hline 66 & 1 & 0 & -1.848665 & 1.665717 & 4.858550 \\
\hline 67 & 1 & 0 & -5.861121 & 1.850665 & 3.322292 \\
\hline 68 & 1 & 0 & -4.242426 & 2.275384 & 5.156485 \\
\hline 69 & 6 & 0 & -3.003501 & -1.931909 & 0.595237 \\
\hline 70 & 6 & 0 & -3.809870 & -2.288888 & 1.682054 \\
\hline 71 & 6 & 0 & -2.539592 & -2.933625 & -0.267750 \\
\hline 72 & 6 & 0 & -4.157708 & -3.620203 & 1.890896 \\
\hline 73 & 1 & 0 & -4.165871 & -1.531872 & 2.378578 \\
\hline 74 & 6 & 0 & -2.898914 & -4.259899 & -0.057046 \\
\hline 75 & 1 & 0 & -1.875384 & -2.677284 & -1.098270 \\
\hline 76 & 6 & 0 & -3.706049 & -4.607382 & 1.021966 \\
\hline 77 & 1 & 0 & -4.782337 & -3.884220 & 2.742851 \\
\hline 78 & 1 & 0 & -2.531146 & -5.024983 & -0.738578 \\
\hline 79 & 1 & 0 & -3.975701 & -5.648649 & 1.191295 \\
\hline 80 & 46 & 0 & -0.085424 & -0.044620 & 0.064316 \\
\hline 81 & 6 & 0 & 1.581200 & -3.005578 & 0.062907 \\
\hline 82 & 1 & 0 & 1.619060 & -2.780388 & -1.005895 \\
\hline 83 & 6 & 0 & 0.821651 & -2.293806 & 0.863023 \\
\hline 84 & 6 & 0 & 0.111134 & -1.769001 & 1.847583 \\
\hline 85 & 1 & 0 & 0.551731 & -1.061706 & 2.550821 \\
\hline 86 & 6 & 0 & 2.406887 & -4.126017 & 0.605481 \\
\hline 87 & 8 & 0 & 2.478563 & -4.376610 & 1.792148 \\
\hline 88 & 6 & 0 & 3.177446 & -4.883479 & -0.441249 \\
\hline 89 & 1 & 0 & 3.746354 & -5.695236 & 0.020153 \\
\hline 90 & 1 & 0 & 3.860517 & -4.195773 & -0.961604 \\
\hline 91 & 1 & 0 & -1.298234 & 5.515642 & -1.649131 \\
\hline 92 & 1 & 0 & -0.879051 & -2.164442 & 2.083712 \\
\hline 93 & 1 & 0 & 2.496691 & -5.283263 & -1.205014 \\
\hline \multicolumn{6}{|c|}{0.736009 (Hartree/Particle) } \\
\hline \multicolumn{6}{|c|}{ Thermal correction to Energy= } \\
\hline \multicolumn{6}{|c|}{ Thermal correction to Enthalpy= } \\
\hline \multicolumn{4}{|c|}{ Thermal correction to Gibbs Free Energy= } & \multicolumn{2}{|c|}{.647636} \\
\hline \multicolumn{4}{|c|}{$\begin{array}{l}\text { Sum of electronic and zero-point Energies= } \\
\text { Sum of electronic and thermal Energies= }\end{array}$} & \multicolumn{2}{|c|}{-2709.439313} \\
\hline \multicolumn{4}{|c|}{ Sum of electronic and thermal Energies= } & \multicolumn{2}{|c|}{-2709.388640} \\
\hline \multirow{2}{*}{\multicolumn{4}{|c|}{$\begin{array}{l}\text { Sum of electronic and thermal Enthalpies= } \\
\text { Sum of electronic and thermal Free Energies= }\end{array}$}} & \multicolumn{2}{|c|}{-2709.387696} \\
\hline & & & & \multicolumn{2}{|c|}{ Sum of electronic and thermal Free Energies= } \\
\hline
\end{tabular}

INT4

\begin{tabular}{rrrrrr} 
Center & Atomic & Atomic & \multicolumn{2}{c}{ Coordinates (Angstroms) } \\
Number & Number & Type & $\mathrm{X}$ & $\mathrm{Y}$ & $\mathrm{Z}$ \\
-------------18 & & \\
1 & 6 & 0 & 0.378318 & 4.890375 & -0.615826 \\
2 & 6 & 0 & -0.101531 & 4.145613 & -1.690432 \\
3 & 6 & 0 & 0.014094 & 2.756587 & -1.689053 \\
4 & 6 & 0 & 0.612199 & 2.103647 & -0.611255 \\
5 & 6 & 0 & 1.090497 & 2.850324 & 0.464971 \\
6 & 6 & 0 & 0.972096 & 4.241039 & 0.462128 \\
7 & 1 & 0 & -0.572655 & 4.646962 & -2.535814 \\
8 & 1 & 0 & -0.386601 & 2.182750 & -2.527996 \\
9 & 1 & 0 & 1.556359 & 2.351843 & 1.318574 \\
10 & 1 & 0 & 1.348480 & 4.816900 & 1.307578 \\
11 & 53 & 0 & 1.291852 & -2.656690 & -0.478613 \\
12 & 46 & 0 & 0.870711 & 0.074727 & -0.607038 \\
13 & 15 & 0 & -1.341142 & -0.110006 & 0.181396 \\
14 & 6 & 0 & 3.806679 & 0.346739 & 0.470392 \\
15 & 1 & 0 & 3.314836 & 0.162945 & 1.426915 \\
16 & 6 & 0 & 3.124217 & 0.356790 & -0.652974 \\
17 & 6 & 0 & 2.737485 & 0.419111 & -1.930031 \\
18 & 1 & 0 & 2.758791 & -0.476863 & -2.551849 \\
19 & 6 & 0 & 5.288869 & 0.539047 & 0.445117 \\
20 & 8 & 0 & 5.895502 & 0.788876 & -0.574092 \\
21 & 6 & 0 & -2.213530 & -1.523433 & -0.568618 \\
22 & 6 & 0 & -2.880415 & -2.479733 & 0.195624 \\
23 & 6 & 0 & -2.160524 & -1.664858 & -1.959544 \\
24 & 6 & 0 & -3.495354 & -3.561760 & -0.427354 \\
25 & 1 & 0 & -2.900984 & -2.397198 & 1.281166 \\
26 & 6 & 0 & -2.788164 & -2.736926 & -2.578415 \\
27 & 1 & 0 & -1.599053 & -0.942593 & -2.556237 \\
28 & 6 & 0 & -3.453228 & -3.689758 & -1.810341 \\
29 & 1 & 0 & -4.001881 & -4.313282 & 0.175607 \\
30 & 1 & 0 & -2.738059 & -2.842840 & -3.660404 \\
31 & 1 & 0 & -3.930286 & -4.540592 & -2.293748 \\
32 & 6 & 0 & -1.390101 & -0.344764 & 1.990140 \\
33 & 6 & 0 & -0.228515 & -0.697114 & 2.680876 \\
34 & 6 & 0 & -2.589165 & -0.183635 & 2.696773 \\
35 & 6 & 0 & -0.265263 & -0.889483 & 4.058673 \\
36 & 1 & 0 & 0.701828 & -0.842231 & 2.129049 \\
37 & 6 & 0 & -2.622420 & -0.380873 & 4.071195 \\
38 & 1 & 0 & -3.498924 & 0.100528 & 2.166958 \\
39 & 6 & 0 & -1.459600 & -0.732431 & 4.753098 \\
40 & 1 & 0 & 0.643552 & -1.167453 & 4.589157 \\
41 & 1 & 0 & -3.558735 & -0.256531 & 4.612446 \\
42 & 1 & 0 & -1.486860 & -0.883130 & 5.831015 \\
43 & 6 & 0 & -2.448493 & 1.322286 & -0.088855 \\
44 & 6 & 0 & -3.378925 & 1.352139 & -1.128972 \\
45 & 6 & 0 & -2.278889 & 2.460761 & 0.708074 \\
46 & 6 & 0 & -4.121165 & 2.504780 & -1.373464 \\
47 & 1 & 0 & -3.535684 & 0.472053 & -1.750624 \\
48 & 6 & 0 & -3.020811 & 3.607067 & 0.462326
\end{tabular}




$\begin{array}{rlrrrr}49 & 1 & 0 & -1.548168 & 2.455294 & 1.516347 \\ 50 & 6 & 0 & -3.941968 & 3.633133 & -0.582189 \\ 51 & 1 & 0 & -4.845800 & 2.514769 & -2.185980 \\ 52 & 1 & 0 & -2.870818 & 4.487931 & 1.084167 \\ 53 & 1 & 0 & -4.519844 & 4.534732 & -0.778599 \\ 54 & 6 & 0 & 5.963949 & 0.393094 & 1.783640 \\ 55 & 1 & 0 & 5.783045 & -0.610836 & 2.191749 \\ 56 & 1 & 0 & 5.546826 & 1.110122 & 2.504168 \\ 57 & 1 & 0 & 7.039309 & 0.559310 & 1.677995 \\ 58 & 1 & 0 & 2.543784 & 1.381436 & -2.405066 \\ 59 & 1 & 0 & 0.287745 & 5.975589 & -0.618754\end{array}$

Zero-point correction=

Thermal correction to Energy=

0.460794 (Hartree/Particle)

Thermal correction to Enthalpy=

0.494211

Thermal correction to Gibbs Free Energy=

Sum of electronic and zero-point Energies=

Sum of electronic and thermal Energies=

0.495155

0.392068

-1673.998888
-1673.965471

$-1673.964527$

Sum of electronic and thermal Free Energies=

$-1674.067615$

M06 /6-311++G(d,p)-SDD/SMD//M06/6-31G(d)-LANL2DZ energy in toluene solvent = -1676.0409614

INT5

\begin{tabular}{|c|c|c|c|c|c|}
\hline \multirow{2}{*}{$\begin{array}{l}\text { Center } \\
\text { Number }\end{array}$} & \multirow{2}{*}{$\begin{array}{l}\text { Atomic } \\
\text { Number }\end{array}$} & \multirow{2}{*}{$\begin{array}{l}\text { Atomic } \\
\text { Type }\end{array}$} & \multicolumn{3}{|c|}{ Coordinates (Angstroms) } \\
\hline & & & $\mathrm{X}$ & $\mathrm{Y}$ & Z \\
\hline 1 & 6 & 0 & -4.177627 & 3.008509 & 1.580419 \\
\hline 2 & 6 & 0 & -3.938681 & 1.679337 & 1.921876 \\
\hline 3 & 6 & 0 & -3.409740 & 0.799301 & 0.986770 \\
\hline 4 & 6 & 0 & -3.104374 & 1.238798 & -0.308208 \\
\hline 5 & 6 & 0 & -3.352162 & 2.574272 & -0.641932 \\
\hline 6 & 6 & 0 & -3.885630 & 3.452571 & 0.294710 \\
\hline 7 & 1 & 0 & -4.153835 & 1.325178 & 2.928549 \\
\hline 8 & 1 & 0 & -3.184078 & -0.225533 & 1.282595 \\
\hline 9 & 1 & 0 & -3.135721 & 2.921297 & -1.652008 \\
\hline 10 & 1 & 0 & -4.078048 & 4.487357 & 0.016421 \\
\hline 11 & 53 & 0 & -0.544300 & -2.679954 & 1.206251 \\
\hline 12 & 46 & 0 & -0.665242 & -0.661905 & -0.598077 \\
\hline 13 & 15 & 0 & 1.358074 & 0.388537 & -0.073483 \\
\hline 14 & 6 & 0 & -2.735547 & -1.063439 & -1.279019 \\
\hline 15 & 1 & 0 & -3.419962 & -1.428094 & -0.511066 \\
\hline 16 & 6 & 0 & -2.488841 & 0.338546 & -1.316153 \\
\hline 17 & 6 & 0 & -1.403185 & 0.799938 & -2.092967 \\
\hline 18 & 1 & 0 & -1.131647 & 0.275051 & -3.006826 \\
\hline 19 & 6 & 0 & -2.477488 & -2.025695 & -2.384125 \\
\hline 20 & 8 & 0 & -1.943196 & -1.725179 & -3.440507 \\
\hline 21 & 6 & 0 & 1.711719 & 1.842648 & -1.133786 \\
\hline 22 & 6 & 0 & 1.751725 & 3.153112 & -0.657618 \\
\hline 23 & 6 & 0 & 1.916652 & 1.602311 & -2.498180 \\
\hline 24 & 6 & 0 & 1.983987 & 4.209788 & -1.536621 \\
\hline 25 & 1 & 0 & 1.599976 & 3.353692 & 0.402450 \\
\hline 26 & 6 & 0 & 2.154741 & 2.655643 & -3.369577 \\
\hline 27 & 1 & 0 & 1.877491 & 0.579414 & -2.878262 \\
\hline 28 & 6 & 0 & 2.184106 & 3.964238 & -2.889561 \\
\hline 29 & 1 & 0 & 2.010681 & 5.229730 & -1.156328 \\
\hline 30 & 1 & 0 & 2.313311 & 2.457441 & -4.428251 \\
\hline 31 & 1 & 0 & 2.363410 & 4.791278 & -3.574456 \\
\hline 32 & 6 & 0 & 1.182140 & 1.057157 & 1.615102 \\
\hline 33 & 6 & 0 & 2.091627 & 0.774461 & 2.634050 \\
\hline 34 & 6 & 0 & 0.038431 & 1.813251 & 1.903790 \\
\hline 35 & 6 & 0 & 1.870028 & 1.257805 & 3.920837 \\
\hline 36 & 1 & 0 & 2.970533 & 0.165485 & 2.426848 \\
\hline 37 & 6 & 0 & -0.172841 & 2.302621 & 3.185478 \\
\hline 38 & 1 & 0 & -0.697748 & 2.015660 & 1.121163 \\
\hline 39 & 6 & 0 & 0.743918 & 2.024010 & 4.197378 \\
\hline 40 & 1 & 0 & 2.582195 & 1.027955 & 4.711474 \\
\hline 41 & 1 & 0 & -1.065080 & 2.890342 & 3.396259 \\
\hline 42 & 1 & 0 & 0.573640 & 2.398876 & 5.205271 \\
\hline 43 & 6 & 0 & 2.945174 & -0.512137 & -0.118727 \\
\hline 44 & 6 & 0 & 2.963663 & -1.903037 & -0.231243 \\
\hline 45 & 6 & 0 & 4.153672 & 0.194078 & -0.051159 \\
\hline 46 & 6 & 0 & 4.178794 & -2.582090 & -0.266911 \\
\hline 47 & 1 & 0 & 2.024049 & -2.453421 & -0.278183 \\
\hline 48 & 6 & 0 & 5.362504 & -0.488179 & -0.083987 \\
\hline 49 & 1 & 0 & 4.144136 & 1.282229 & 0.025286 \\
\hline 50 & 6 & 0 & 5.374526 & -1.877603 & -0.193187 \\
\hline 51 & 1 & 0 & 4.185661 & -3.666813 & -0.354137 \\
\hline 52 & 1 & 0 & 6.298757 & 0.064939 & -0.030402 \\
\hline 53 & 1 & 0 & 6.323521 & -2.410754 & -0.223912 \\
\hline 54 & 6 & 0 & -2.946419 & -3.427893 & -2.101751 \\
\hline 55 & 1 & 0 & -4.038498 & -3.448414 & -1.981504 \\
\hline 56 & 1 & 0 & -2.510369 & -3.790595 & -1.159606 \\
\hline 57 & 1 & 0 & -2.659116 & -4.085571 & -2.926562 \\
\hline 58 & 1 & 0 & -4.592459 & 3.696744 & 2.315111 \\
\hline 59 & 1 & 0 & -1.066549 & 1.831120 & -1.977616 \\
\hline
\end{tabular}

Zero-point correction $=$ 0.464695 (Hartree/Particle)

Thermal correction to Energy=

Thermal correction to Enthalpy=

Thermal correction to Gibbs Free Energy=

Sum of electronic and zero-point Energies

Sum of electronic and thermal Energies=

0.495915

0.496859

0.399261

$-1674.076951$

$-1674.045731$ 
Sum of electronic and thermal Enthalpies $=$

$-1674.044787$

Sum of electronic and thermal Free Energies= $\quad-1674.142385$

M06 /6-311++G(d,p)-SDD/SMD//M06/6-31G(d)-LANL2DZ energy in toluene solvent = - 1676.1143713

INT5-a

\begin{tabular}{|c|c|c|c|c|c|}
\hline \multirow{2}{*}{$\begin{array}{l}\text { Center } \\
\text { Number }\end{array}$} & \multirow{2}{*}{$\begin{array}{l}\text { Atomic } \\
\text { Number }\end{array}$} & \multirow{2}{*}{$\begin{array}{l}\text { Atomic } \\
\text { Type }\end{array}$} & \multicolumn{3}{|c|}{ Coordinates (Angstroms) } \\
\hline & & & $\mathrm{X}$ & $\mathrm{Y}$ & a \\
\hline 1 & 6 & 0 & 5.212955 & 2.300406 & -1.732165 \\
\hline 2 & 6 & 0 & 4.870188 & 0.950289 & -1.771980 \\
\hline 3 & 6 & 0 & 3.995797 & 0.414418 & -0.835321 \\
\hline 4 & 6 & 0 & 3.444445 & 1.223651 & 0.167371 \\
\hline 5 & 6 & 0 & 3.794272 & 2.578139 & 0.195609 \\
\hline 6 & 6 & 0 & 4.669464 & 3.113779 & -0.743572 \\
\hline 7 & 1 & 0 & 5.276412 & 0.306617 & -2.551537 \\
\hline 8 & 1 & 0 & 3.696959 & -0.632720 & -0.903948 \\
\hline 9 & 1 & 0 & 3.381558 & 3.211362 & 0.981374 \\
\hline 10 & 1 & 0 & 4.931123 & 4.170977 & -0.697909 \\
\hline 11 & 53 & 0 & 1.115968 & -2.687713 & -1.056579 \\
\hline 12 & 46 & 0 & 0.736819 & -0.345310 & 0.381749 \\
\hline 13 & 15 & 0 & -1.463250 & 0.319723 & -0.052627 \\
\hline 14 & 6 & 0 & 2.638680 & -0.622585 & 1.700167 \\
\hline 15 & 1 & 0 & 3.437975 & -1.231485 & 1.275455 \\
\hline 16 & 6 & 0 & 2.511489 & 0.694423 & 1.194732 \\
\hline 17 & 6 & 0 & 1.343221 & 1.419513 & 1.539580 \\
\hline 18 & 1 & 0 & 0.884185 & 1.182792 & 2.500838 \\
\hline 19 & 6 & 0 & 2.005760 & -1.161046 & 2.941915 \\
\hline 20 & 8 & 0 & 1.063815 & -0.540068 & 3.531634 \\
\hline 21 & 6 & 0 & -2.202363 & 1.113458 & 1.430570 \\
\hline 22 & 6 & 0 & -3.179252 & 2.112246 & 1.366709 \\
\hline 23 & 6 & 0 & -1.770380 & 0.650917 & 2.679994 \\
\hline 24 & 6 & 0 & -3.724513 & 2.638316 & 2.533287 \\
\hline 25 & 1 & 0 & -3.515155 & 2.485113 & 0.398052 \\
\hline 26 & 6 & 0 & -2.322160 & 1.180903 & 3.843348 \\
\hline 27 & 1 & 0 & -0.962238 & -0.085472 & 2.767302 \\
\hline 28 & 6 & 0 & -3.298208 & 2.169889 & 3.773904 \\
\hline 29 & 1 & 0 & -4.481914 & 3.419803 & 2.472262 \\
\hline 30 & 1 & 0 & -1.962447 & 0.820930 & 4.806051 \\
\hline 31 & 1 & 0 & -3.721353 & 2.586639 & 4.687992 \\
\hline 32 & 6 & 0 & -1.619384 & 1.589809 & -1.375884 \\
\hline 33 & 6 & 0 & -2.818102 & 1.840242 & -2.053403 \\
\hline 34 & 6 & 0 & -0.483757 & 2.330229 & -1.715742 \\
\hline 35 & 6 & 0 & -2.884320 & 2.827908 & -3.030387 \\
\hline 36 & 1 & 0 & -3.703231 & 1.246871 & -1.818181 \\
\hline 37 & 6 & 0 & -0.550804 & 3.320283 & -2.691197 \\
\hline 38 & 1 & 0 & 0.463606 & 2.104266 & -1.221819 \\
\hline 39 & 6 & 0 & -1.751437 & 3.572862 & -3.347197 \\
\hline 40 & 1 & 0 & -3.823672 & 3.012258 & -3.551091 \\
\hline 41 & 1 & 0 & 0.344108 & 3.885606 & -2.947845 \\
\hline 42 & 1 & 0 & -1.803171 & 4.342875 & -4.116465 \\
\hline 43 & 6 & 0 & -2.761933 & -0.879401 & -0.549959 \\
\hline 44 & 6 & 0 & -2.503099 & -1.683155 & -1.666916 \\
\hline 45 & 6 & 0 & -3.974910 & -1.025391 & 0.127527 \\
\hline 46 & 6 & 0 & -3.448895 & -2.601168 & -2.105062 \\
\hline 47 & 1 & 0 & -1.540119 & -1.605649 & -2.174515 \\
\hline 48 & 6 & 0 & -4.918521 & -1.950784 & -0.311974 \\
\hline 49 & 1 & 0 & -4.185547 & -0.414118 & 1.004950 \\
\hline 50 & 6 & 0 & -4.659368 & -2.736635 & -1.429391 \\
\hline 51 & 1 & 0 & -3.229676 & -3.225378 & -2.970144 \\
\hline 52 & 1 & 0 & -5.860408 & -2.057374 & 0.225698 \\
\hline 53 & 1 & 0 & -5.397061 & -3.462727 & -1.770379 \\
\hline 54 & 6 & 0 & 2.620028 & -2.329597 & 3.353301 \\
\hline 55 & 1 & 0 & 2.305633 & -2.809202 & 4.279446 \\
\hline 56 & 1 & 0 & 3.387432 & -2.818646 & 2.757550 \\
\hline 57 & 1 & 0 & 5.898593 & 2.715220 & -2.470792 \\
\hline 58 & 1 & 0 & 1.167271 & 2.397670 & 1.08504 \\
\hline
\end{tabular}

Zero-point correction

0.450947 (Hartree/Particle)

Thermal correction to Energy

Thermal correction to Enthalpy=

$\quad 0.383612$

Sum of electronic and zero-point Energies= $\quad-1673.503540$

Sum of electronic and thermal Energies=

$$
\begin{aligned}
& -1673.503540 \\
& -1673.471732 \\
& -1673.470788
\end{aligned}
$$

$-1673.570875$

Sum of electron and thermal Free Energies=

DZ energy in toluene solvent $=-1675.5709789$

\section{INT5-b}

\begin{tabular}{rrrrrr} 
Center & Atomic & Atomic & \multicolumn{2}{c}{ Coordinates (Angstroms) } & $\mathrm{Z}$ \\
Number & Number & Type & $\mathrm{X}$ & $\mathrm{Y}$ & \\
- & - & 0 & -8.910172 & 0.197754 & -0.538624 \\
2 & 6 & 0 & -8.268538 & 1.251858 & 0.104286 \\
3 & 6 & 0 & -6.880446 & 1.294451 & 0.160935 \\
4 & 6 & 0 & -6.104947 & 0.290689 & -0.430225 \\
5 & 6 & 0 & -6.764133 & -0.767491 & -1.065163 \\
6 & 6 & 0 & -8.151628 & -0.813041 & -1.121655 \\
7 & 6 & 0 & -8.852030 & 2.050019 & 0.561187 \\
8 & 1 & 0 & -6.382336 & 2.132443 & 0.649151 \\
9 & 1 & 0 & -6.173920 & -1.574333 & -1.498794 \\
10 & 1 & 0 & -8.644465 & -1.649393 & -1.615615
\end{tabular}




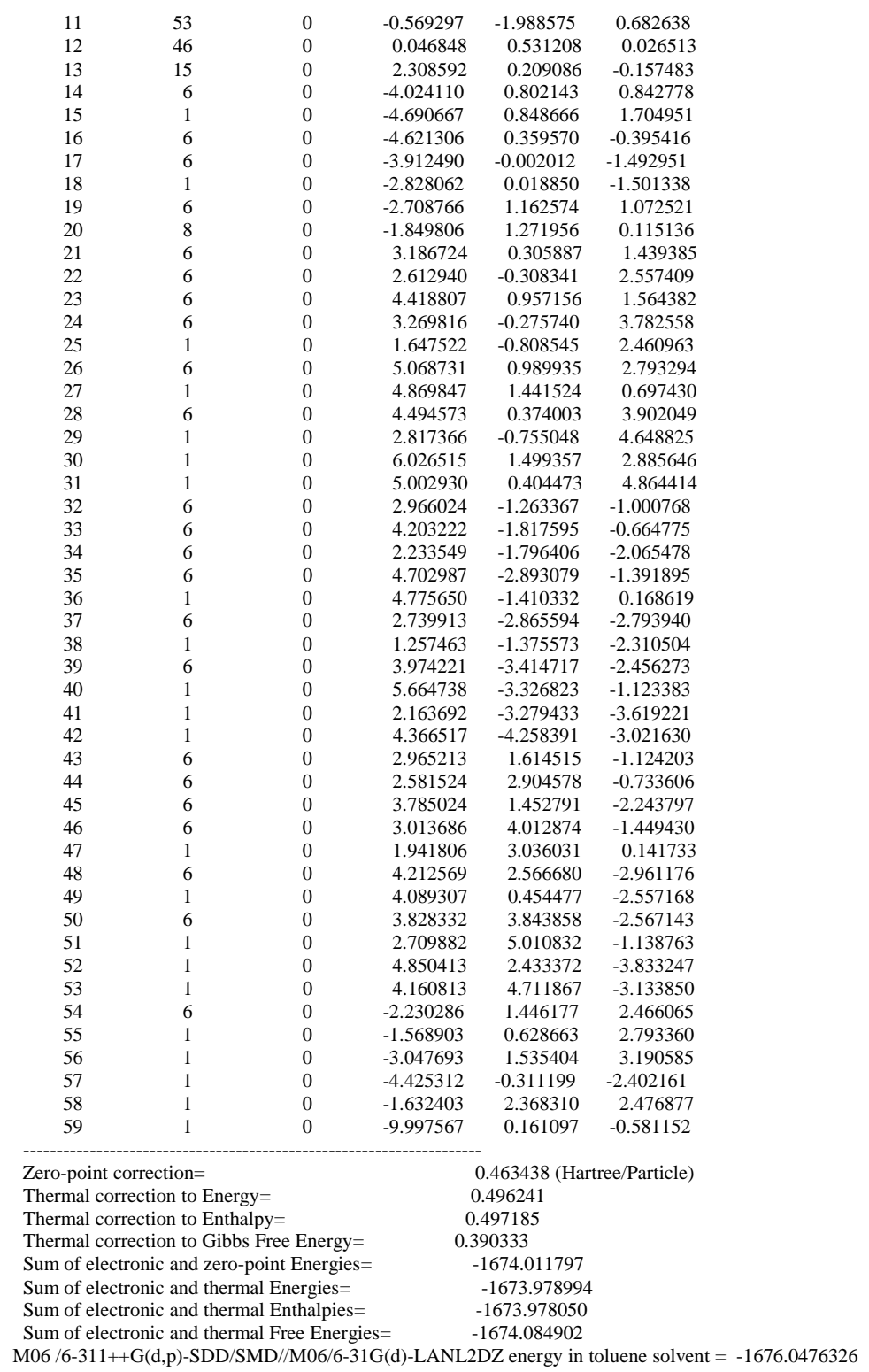

INT6

\begin{tabular}{|c|c|c|c|c|c|}
\hline \multirow{2}{*}{$\begin{array}{l}\text { Center } \\
\text { Number }\end{array}$} & \multirow{2}{*}{$\begin{array}{l}\text { Atomic } \\
\text { Number }\end{array}$} & \multirow{2}{*}{$\begin{array}{l}\text { Atomic } \\
\text { Type }\end{array}$} & \multicolumn{3}{|c|}{ Coordinates (Angstroms) } \\
\hline & & & $\mathrm{X}$ & $\mathrm{Y}$ & \\
\hline 1 & 6 & 0 & 0.179398 & 4.249905 & -0.405291 \\
\hline 2 & 6 & 0 & 0.093922 & 3.566527 & 0.790313 \\
\hline 3 & 6 & 0 & -0.919731 & 2.617278 & 0.990378 \\
\hline 4 & 6 & 0 & -1.893178 & 2.375875 & -0.022649 \\
\hline 5 & 6 & 0 & -1.741515 & 3.058250 & -1.260881 \\
\hline 6 & 6 & 0 & -0.740159 & 3.986933 & -1.435496 \\
\hline 7 & 1 & 0 & 0.819641 & 3.750438 & 1.581082 \\
\hline 8 & 1 & 0 & -1.048626 & 2.177461 & 1.980375 \\
\hline 9 & 1 & 0 & -2.456744 & 2.864386 & -2.058487 \\
\hline 10 & 1 & 0 & -0.662662 & 4.522605 & -2.380141 \\
\hline 11 & 53 & 0 & -1.921110 & -2.109654 & -0.283303 \\
\hline 12 & 46 & 0 & -0.838483 & 0.397231 & -0.056872 \\
\hline 13 & 15 & 0 & 1.471275 & -0.229970 & -0.016161 \\
\hline 14 & 6 & 0 & -3.902173 & 0.939304 & -0.755166 \\
\hline 15 & 1 & 0 & -3.875092 & 1.035215 & -1.834477 \\
\hline 16 & 6 & 0 & -2.871647 & 1.334804 & 0.167761 \\
\hline 17 & 6 & 0 & -3.434692 & 0.968587 & 1.527317 \\
\hline 18 & 1 & 0 & -2.770369 & 0.361742 & 2.154677 \\
\hline 19 & 6 & 0 & -4.836759 & 0.245794 & -0.065995 \\
\hline 20 & 8 & 0 & -4.607829 & 0.200453 & 1.263504 \\
\hline 21 & 6 & 0 & 2.605491 & 1.183715 & 0.269344 \\
\hline 22 & 6 & 0 & 3.313036 & 1.371011 & 1.459377 \\
\hline 23 & 6 & 0 & 2.696615 & 2.157100 & -0.733276 \\
\hline 24 & 6 & 0 & 4.103792 & 2.504257 & 1.636905 \\
\hline 25 & 1 & 0 & 3.253807 & 0.624882 & 2.251440 \\
\hline 26 & 6 & 0 & 3.503212 & 3.275485 & -0.562827 \\
\hline 27 & 1 & 0 & 2.134471 & 2.028319 & -1.660994 \\
\hline 28 & 6 & 0 & 4.206038 & 3.454879 & 0.626618 \\
\hline
\end{tabular}




$\begin{array}{rrrrrr}29 & 1 & 0 & 4.652879 & 2.635919 & 2.568237 \\ 30 & 1 & 0 & 3.578299 & 4.015069 & -1.359428 \\ 31 & 1 & 0 & 4.834076 & 4.333640 & 0.763883 \\ 32 & 6 & 0 & 1.856053 & -1.350175 & 1.380127 \\ 33 & 6 & 0 & 2.987957 & -2.168366 & 1.408103 \\ 34 & 6 & 0 & 1.004156 & -1.325135 & 2.487401 \\ 35 & 6 & 0 & 3.265688 & -2.941763 & 2.529928 \\ 36 & 1 & 0 & 3.653142 & -2.205633 & 0.545368 \\ 37 & 6 & 0 & 1.286778 & -2.092069 & 3.612243 \\ 38 & 1 & 0 & 0.101798 & -0.710066 & 2.451455 \\ 39 & 6 & 0 & 2.418279 & -2.901326 & 3.633663 \\ 40 & 1 & 0 & 4.146935 & -3.581314 & 2.540756 \\ 41 & 1 & 0 & 0.614073 & -2.068965 & 4.467812 \\ 42 & 1 & 0 & 2.637410 & -3.509325 & 4.510047 \\ 43 & 6 & 0 & 2.219797 & -1.025119 & -1.489540 \\ 44 & 6 & 0 & 3.590348 & -0.932252 & -1.765093 \\ 45 & 6 & 0 & 1.399753 & -1.763726 & -2.346710 \\ 46 & 6 & 0 & 4.130300 & -1.577759 & -2.871387 \\ 47 & 1 & 0 & 4.236708 & -0.342012 & -1.114363 \\ 48 & 6 & 0 & 1.945108 & -2.409792 & -3.452261 \\ 49 & 1 & 0 & 0.331183 & -1.837235 & -2.139853 \\ 50 & 6 & 0 & 3.307610 & -2.319175 & -3.715419 \\ 51 & 1 & 0 & 5.196967 & -1.499250 & -3.076411 \\ 52 & 1 & 0 & 1.297083 & -2.986126 & -4.110356 \\ 53 & 1 & 0 & 3.731229 & -2.823217 & -4.582941 \\ 54 & 6 & 0 & -6.021117 & -0.504032 & -0.528750 \\ 55 & 1 & 0 & -5.865693 & -1.576761 & -0.350305 \\ 56 & 1 & 0 & -6.187642 & -0.349393 & -1.599056 \\ 57 & 1 & 0 & 0.967446 & 4.985997 & -0.554361 \\ 58 & 1 & 0 & -6.918846 & -0.203380 & 0.026430 \\ 59 & 1 & 0 & -3.733084 & 1.867881 & 2.092740\end{array}$

Zero-point correction=

0.466118 (Hartree/Particle)

Thermal correction to Energy=

Thermal correction to Enthalpy=

Thermal correction to Gibbs Free Energy=

Sum of electronic and zero-point Energies=

Sum of electronic and thermal Energies $=$

Sum of electronic and thermal Enthalpies=

Sum of electronic and thermal Free Energies=

0.497954

0.498898
0.398535

$-1674.057616$

$-1674.025780$

$-1674.024836$

$-1674.125199$

M06 /6-311++G(d,p)-SDD/SMD//M06/6-31G(d)-LANL2DZ energy in toluene solvent $=-1676.0926648$

INT6

\begin{tabular}{|c|c|c|c|c|c|}
\hline \multirow{2}{*}{$\begin{array}{l}\text { Center } \\
\text { Number }\end{array}$} & \multirow{2}{*}{$\begin{array}{l}\text { Atomic } \\
\text { Number }\end{array}$} & \multirow{2}{*}{$\begin{array}{l}\text { Atomic } \\
\text { Type }\end{array}$} & \multicolumn{3}{|c|}{ Coordinates (Angstroms) } \\
\hline & & & $\mathrm{X}$ & $\mathrm{Y}$ & Z \\
\hline 1 & 6 & 0 & -4.000581 & 2.588672 & 0.207400 \\
\hline 2 & 6 & 0 & -3.542942 & 1.987700 & 1.377702 \\
\hline 3 & 6 & 0 & -2.552185 & 1.005531 & 1.321341 \\
\hline 4 & 6 & 0 & -2.017950 & 0.612730 & 0.093082 \\
\hline 5 & 6 & 0 & -2.473828 & 1.224491 & -1.075675 \\
\hline 6 & 6 & 0 & -3.462819 & 2.204655 & -1.019703 \\
\hline 7 & 1 & 0 & -3.955717 & 2.284320 & 2.342155 \\
\hline 8 & 1 & 0 & -2.202476 & 0.540130 & 2.245495 \\
\hline 9 & 1 & 0 & -2.046153 & 0.946773 & -2.041814 \\
\hline 10 & 1 & 0 & -3.810374 & 2.675138 & -1.939577 \\
\hline 11 & 53 & 0 & 1.234024 & -2.937193 & 0.187912 \\
\hline 12 & 46 & 0 & -0.639762 & -0.878294 & 0.052470 \\
\hline 13 & 15 & 0 & 0.995309 & 0.777482 & -0.040405 \\
\hline 14 & 6 & 0 & -3.533411 & -2.081659 & -1.305912 \\
\hline 15 & 1 & 0 & -3.857735 & -1.990274 & -2.335190 \\
\hline 16 & 6 & 0 & -2.299796 & -2.605040 & -0.829709 \\
\hline 17 & 6 & 0 & -2.323313 & -2.458567 & 0.548406 \\
\hline 18 & 1 & 0 & -1.521837 & -3.110624 & -1.390732 \\
\hline 19 & 6 & 0 & -4.229547 & -1.672535 & -0.206703 \\
\hline 20 & 8 & 0 & -3.525615 & -1.921884 & 0.920696 \\
\hline 21 & 6 & 0 & 1.787883 & 1.067293 & 1.576286 \\
\hline 22 & 6 & 0 & 1.611019 & 0.143104 & 2.607966 \\
\hline 23 & 6 & 0 & 2.579295 & 2.202560 & 1.793826 \\
\hline 24 & 6 & 0 & 2.223626 & 0.346796 & 3.840821 \\
\hline 25 & 1 & 0 & 1.006125 & -0.746649 & 2.428392 \\
\hline 26 & 6 & 0 & 3.191509 & 2.400181 & 3.025037 \\
\hline 27 & 1 & 0 & 2.711097 & 2.935332 & 0.996830 \\
\hline 28 & 6 & 0 & 3.012781 & 1.472532 & 4.049160 \\
\hline 29 & 1 & 0 & 2.083867 & -0.381169 & 4.637724 \\
\hline 30 & 1 & 0 & 3.807243 & 3.283000 & 3.188310 \\
\hline 31 & 1 & 0 & 3.490698 & 1.630779 & 5.014766 \\
\hline 32 & 6 & 0 & 2.312268 & 0.307813 & -1.211623 \\
\hline 33 & 6 & 0 & 1.928609 & -0.153737 & -2.475312 \\
\hline 34 & 6 & 0 & 3.664207 & 0.344954 & -0.874483 \\
\hline 35 & 6 & 0 & 2.886689 & -0.554749 & -3.395701 \\
\hline 36 & 1 & 0 & 0.867619 & -0.222248 & -2.725480 \\
\hline 37 & 6 & 0 & 4.621881 & -0.071639 & -1.794751 \\
\hline 38 & 1 & 0 & 3.972229 & 0.675935 & 0.116224 \\
\hline 39 & 6 & 0 & 4.236306 & -0.518492 & -3.052812 \\
\hline 40 & 1 & 0 & 2.579076 & -0.917732 & -4.374652 \\
\hline 41 & 1 & 0 & 5.675048 & -0.055617 & -1.519837 \\
\hline 42 & 1 & 0 & 4.987521 & -0.852222 & -3.766664 \\
\hline 43 & 6 & 0 & 0.495390 & 2.459974 & -0.569051 \\
\hline 44 & 6 & 0 & -0.244125 & 3.252169 & 0.317823 \\
\hline 45 & 6 & 0 & 0.741074 & 2.933846 & -1.858914 \\
\hline 46 & 6 & 0 & -0.739124 & 4.483793 & -0.084271 \\
\hline
\end{tabular}




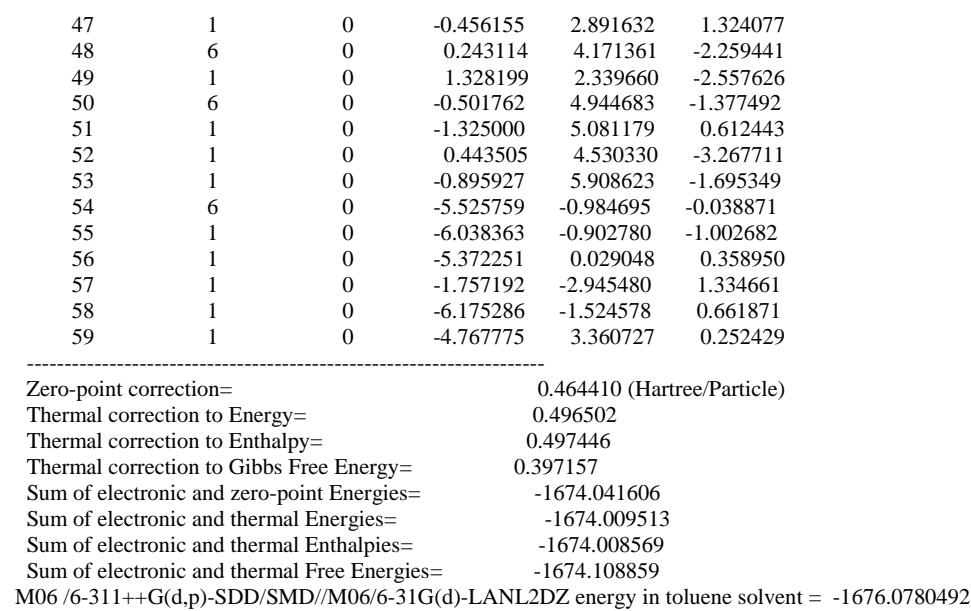

INT6"

\begin{tabular}{|c|c|c|c|c|c|}
\hline \multirow{2}{*}{$\begin{array}{l}\text { Center } \\
\text { Number }\end{array}$} & \multirow{2}{*}{$\begin{array}{l}\text { Atomic } \\
\text { Number }\end{array}$} & \multirow{2}{*}{$\begin{array}{l}\text { Atomic } \\
\text { Type }\end{array}$} & \multicolumn{3}{|c|}{ Coordinates (Angstroms) } \\
\hline & & & $\mathrm{X}$ & $\mathrm{Y}$ & Z \\
\hline 1 & 6 & 0 & -0.036822 & 5.057596 & 0.384956 \\
\hline 2 & 6 & 0 & 0.164460 & 4.615274 & -0.921186 \\
\hline 3 & 6 & 0 & 0.321440 & 3.257874 & -1.190253 \\
\hline 4 & 6 & 0 & 0.255135 & 2.306282 & -0.164769 \\
\hline 5 & 6 & 0 & 0.056842 & 2.765610 & 1.141386 \\
\hline 6 & 6 & 0 & -0.082224 & 4.127195 & 1.417101 \\
\hline 7 & 1 & 0 & 0.211670 & 5.335548 & -1.738584 \\
\hline 8 & 1 & 0 & 0.503736 & 2.933784 & -2.215486 \\
\hline 9 & 1 & 0 & -0.012502 & 2.052603 & 1.967820 \\
\hline 10 & 1 & 0 & -0.240807 & 4.457495 & 2.444194 \\
\hline 11 & 53 & 0 & 0.644998 & -2.424119 & -1.317317 \\
\hline 12 & 46 & 0 & 0.381379 & 0.329075 & -0.622705 \\
\hline 13 & 15 & 0 & -1.916603 & -0.069514 & 0.011704 \\
\hline 14 & 6 & 0 & 3.239329 & 1.849247 & -0.716019 \\
\hline 15 & 1 & 0 & 3.023047 & 2.637720 & 0.001078 \\
\hline 16 & 6 & 0 & 2.351734 & 0.775149 & -1.113035 \\
\hline 17 & 6 & 0 & 3.064268 & 0.119843 & -2.076707 \\
\hline 18 & 1 & 0 & 2.602378 & -0.477550 & 0.556677 \\
\hline 19 & 6 & 0 & 4.384919 & 1.748540 & -1.442749 \\
\hline 20 & 8 & 0 & 4.294718 & 0.689832 & -2.293774 \\
\hline 21 & 6 & 0 & -2.991417 & -0.941976 & -1.188831 \\
\hline 22 & 6 & 0 & -2.578908 & -1.016101 & -2.520271 \\
\hline 23 & 6 & 0 & -4.250842 & -1.433490 & -0.830341 \\
\hline 24 & 6 & 0 & -3.414706 & -1.576120 & -3.481903 \\
\hline 25 & 1 & 0 & -1.588618 & -0.651496 & -2.794166 \\
\hline 26 & 6 & 0 & -5.078282 & -2.003837 & -1.789165 \\
\hline 27 & 1 & 0 & -4.589093 & -1.367307 & 0.204501 \\
\hline 28 & 6 & 0 & -4.660686 & -2.072610 & -3.116873 \\
\hline 29 & 1 & 0 & -3.083639 & -1.632334 & -4.517290 \\
\hline 30 & 1 & 0 & -6.055201 & -2.389780 & -1.501825 \\
\hline 31 & 1 & 0 & -5.313072 & -2.515133 & -3.868292 \\
\hline 32 & 6 & 0 & -1.912604 & -1.106849 & 1.523852 \\
\hline 33 & 6 & 0 & -1.638481 & -0.513380 & 2.763828 \\
\hline 34 & 6 & 0 & -2.007496 & -2.499737 & 1.449550 \\
\hline 35 & 6 & 0 & -1.490884 & -1.292707 & 3.906629 \\
\hline 36 & 1 & 0 & -1.557083 & 0.572664 & 2.837651 \\
\hline 37 & 6 & 0 & -1.850344 & -3.278640 & 2.592599 \\
\hline 38 & 1 & 0 & -2.186331 & -2.979198 & 0.487920 \\
\hline 39 & 6 & 0 & -1.599575 & -2.679800 & 3.823535 \\
\hline 40 & 1 & 0 & -1.298117 & -0.814691 & 4.866671 \\
\hline 41 & 1 & 0 & -1.929376 & -4.361921 & 2.517393 \\
\hline 42 & 1 & 0 & -1.492438 & -3.291494 & 4.718635 \\
\hline 43 & 6 & 0 & -3.010839 & 1.346014 & 0.443334 \\
\hline 44 & 6 & 0 & -2.947954 & 2.463630 & -0.394143 \\
\hline 45 & 6 & 0 & -3.934285 & 1.338742 & 1.492200 \\
\hline 46 & 6 & 0 & -3.772964 & 3.559739 & -0.176185 \\
\hline 47 & 1 & 0 & -2.225580 & 2.484867 & -1.209749 \\
\hline 48 & 6 & 0 & -4.759852 & 2.437830 & 1.711082 \\
\hline 49 & 1 & 0 & -4.010085 & 0.473394 & 2.150005 \\
\hline 50 & 6 & 0 & -4.677574 & 3.551118 & 0.881380 \\
\hline 51 & 1 & 0 & -3.699012 & 4.427557 & -0.829467 \\
\hline 52 & 1 & 0 & -5.471053 & 2.421346 & 2.535880 \\
\hline 53 & 1 & 0 & -5.319108 & 4.412739 & 1.060081 \\
\hline 54 & 6 & 0 & 5.640245 & 2.533255 & -1.490039 \\
\hline 55 & 1 & 0 & 5.588384 & 3.363452 & -0.776684 \\
\hline 56 & 1 & 0 & 5.816926 & 2.955320 & -2.488973 \\
\hline 57 & 1 & 0 & 2.848776 & -0.748974 & -2.687081 \\
\hline 58 & 1 & 0 & 6.518610 & 1.920509 & -1.239284 \\
\hline 59 & 1 & 0 & -0.153463 & 6.120049 & 0.595348 \\
\hline 60 & 7 & 0 & 3.010428 & -0.944541 & 1.409508 \\
\hline 61 & 6 & 0 & 1.832208 & -1.497988 & 2.168048 \\
\hline 62 & 1 & 0 & 1.386263 & -2.246319 & 1.499972 \\
\hline 63 & 6 & 0 & 3.889112 & -2.058795 & 0.906251 \\
\hline 64 & 1 & 0 & 3.235235 & -2.657126 & 0.256324 \\
\hline
\end{tabular}




$\begin{array}{rrrrrr}65 & 1 & 0 & 4.163160 & -2.660210 & 1.782091 \\ 66 & 6 & 0 & 3.776607 & 0.106466 & 2.154485 \\ 67 & 1 & 0 & 4.462680 & 0.546223 & 1.421783 \\ 68 & 1 & 0 & 4.368306 & -0.413161 & 2.921173 \\ 69 & 1 & 0 & 1.098218 & -0.684984 & 2.243053 \\ 70 & 6 & 0 & 2.903514 & 1.191500 & 2.739534 \\ 71 & 1 & 0 & 2.245671 & 0.833145 & 3.540940 \\ 72 & 1 & 0 & 3.550192 & 1.968264 & 3.163825 \\ 73 & 1 & 0 & 2.282791 & 1.663769 & 1.967494 \\ 74 & 6 & 0 & 5.120042 & -1.599828 & 0.157662 \\ 75 & 1 & 0 & 5.846459 & -1.091434 & 0.803015 \\ 76 & 1 & 0 & 5.614839 & -2.487408 & -0.253216 \\ 77 & 1 & 0 & 4.868903 & -0.947205 & -0.686749 \\ 78 & 6 & 0 & 2.175233 & -2.075544 & 3.521019 \\ 79 & 1 & 0 & 2.602154 & -1.336581 & 4.210749 \\ 80 & 1 & 0 & 1.243459 & -2.442733 & 3.967960 \\ 81 & 1 & 0 & 2.862588 & -2.927731 & 3.459137 \\ & 1 & & & & \end{array}$

Zero-point correction=

0.676524 (Hartree/Particle)

Thermal correction to Energy=

0.718322

correction to Enthalpy=

0.719266

0.600642

Sum of ecction to Gibbs Free Energy

Sum of electronic and thermal Energies=

Sum of electronic and thermal Enthalpies=

$-1965.991186$

$-1965.949388$

-1965.949388
-1965.948444

$-1966.067068$

Sum of electronic and thermal Free Energies=

M06 /6-311++G(d,p)-SDD/SMD//M06/6-31G(d)-LANL2DZ energy in toluene solvent $=-1968.3362874$

INT7

\begin{tabular}{|c|c|c|c|c|c|}
\hline \multirow{2}{*}{$\begin{array}{l}\text { Center } \\
\text { Number }\end{array}$} & \multirow{2}{*}{$\begin{array}{l}\text { Atomic } \\
\text { Number }\end{array}$} & \multirow{2}{*}{$\begin{array}{l}\text { Atomic } \\
\text { Type }\end{array}$} & \multicolumn{3}{|c|}{ Coordinates (Angstroms) } \\
\hline & & & $\mathrm{X}$ & $\mathrm{Y}$ & 2 \\
\hline 1 & 53 & 0 & 1.808784 & -3.315634 & -0.187515 \\
\hline 2 & 46 & 0 & 0.009677 & -1.306584 & -0.215183 \\
\hline 3 & 15 & 0 & 1.636249 & 0.565278 & 0.011004 \\
\hline 4 & 1 & 0 & -0.866014 & -2.611170 & -0.350461 \\
\hline 5 & 15 & 0 & -2.071069 & -0.262872 & -0.078282 \\
\hline 6 & 6 & 0 & 3.224546 & 0.272770 & -0.847793 \\
\hline 7 & 6 & 0 & 3.182189 & -0.391911 & -2.076686 \\
\hline 8 & 6 & 0 & 4.443246 & 0.754450 & -0.367768 \\
\hline 9 & 6 & 0 & 4.341890 & -0.556571 & -2.824969 \\
\hline 10 & 1 & 0 & 2.232743 & -0.790197 & -2.438397 \\
\hline 11 & 6 & 0 & 5.605518 & 0.575696 & -1.111329 \\
\hline 12 & 1 & 0 & 4.487749 & 1.267348 & 0.593268 \\
\hline 13 & 6 & 0 & 5.554620 & -0.074911 & -2.340614 \\
\hline 14 & 1 & 0 & 4.300527 & -1.078312 & -3.779386 \\
\hline 15 & 1 & 0 & 6.554869 & 0.946804 & -0.728215 \\
\hline 16 & 1 & 0 & 6.465986 & -0.214182 & -2.920068 \\
\hline 17 & 6 & 0 & 1.185826 & 2.238074 & -0.615419 \\
\hline 18 & 6 & 0 & 0.204488 & 2.985855 & 0.046954 \\
\hline 19 & 6 & 0 & 1.708645 & 2.730996 & -1.815292 \\
\hline 20 & 6 & 0 & -0.244713 & 4.191803 & -0.477105 \\
\hline 21 & 1 & 0 & -0.210427 & 2.623812 & 0.987221 \\
\hline 22 & 6 & 0 & 1.255029 & 3.938337 & -2.340277 \\
\hline 23 & 1 & 0 & 2.481224 & 2.172223 & -2.342838 \\
\hline 24 & 6 & 0 & 0.276656 & 4.670292 & -1.676211 \\
\hline 25 & 1 & 0 & -1.010114 & 4.754640 & 0.056237 \\
\hline 26 & 1 & 0 & 1.676277 & 4.310296 & -3.273331 \\
\hline 27 & 1 & 0 & -0.077324 & 5.613487 & -2.089377 \\
\hline 28 & 6 & 0 & 2.094278 & 0.851912 & 1.763458 \\
\hline 29 & 6 & 0 & 2.405136 & 2.112833 & 2.281228 \\
\hline 30 & 6 & 0 & 2.125814 & -0.261920 & 2.610541 \\
\hline 31 & 6 & 0 & 2.721625 & 2.260753 & 3.627903 \\
\hline 32 & 1 & 0 & 2.393189 & 2.987174 & 1.630706 \\
\hline 33 & 6 & 0 & 2.449804 & -0.111683 & 3.954842 \\
\hline 34 & 1 & 0 & 1.897344 & -1.250784 & 2.205755 \\
\hline 35 & 6 & 0 & 2.740094 & 1.150282 & 4.466826 \\
\hline 36 & 1 & 0 & 2.957049 & 3.248170 & 4.022218 \\
\hline 37 & 1 & 0 & 2.473386 & -0.985885 & 4.603338 \\
\hline 38 & 1 & 0 & 2.986996 & 1.267829 & 5.521020 \\
\hline 39 & 6 & 0 & -2.583225 & 0.715170 & -1.538402 \\
\hline 40 & 6 & 0 & -3.926136 & 1.017573 & -1.791972 \\
\hline 41 & 6 & 0 & -1.600601 & 1.176195 & -2.417837 \\
\hline 42 & 6 & 0 & -4.273487 & 1.787989 & -2.895683 \\
\hline 43 & 1 & 0 & -4.702989 & 0.638179 & -1.126566 \\
\hline 44 & 6 & 0 & -1.950140 & 1.948347 & -3.520446 \\
\hline 45 & 1 & 0 & -0.555800 & 0.917850 & -2.238839 \\
\hline 46 & 6 & 0 & -3.285605 & 2.256163 & -3.758498 \\
\hline 47 & 1 & 0 & -5.320537 & 2.016550 & -3.088391 \\
\hline 48 & 1 & 0 & -1.175202 & 2.301306 & -4.198577 \\
\hline 49 & 1 & 0 & -3.560770 & 2.854782 & -4.625514 \\
\hline 50 & 6 & 0 & -3.525778 & -1.343933 & 0.203283 \\
\hline 51 & 6 & 0 & -4.464553 & -1.096730 & 1.206373 \\
\hline 52 & 6 & 0 & -3.715090 & -2.429214 & -0.658731 \\
\hline 53 & 6 & 0 & -5.574697 & -1.926983 & 1.346451 \\
\hline 54 & 1 & 0 & -4.330524 & -0.256191 & 1.886651 \\
\hline 55 & 6 & 0 & -4.828969 & -3.246494 & -0.525583 \\
\hline 56 & 1 & 0 & -2.975462 & -2.637208 & -1.433675 \\
\hline 57 & 6 & 0 & -5.760140 & -2.998087 & 0.481020 \\
\hline 58 & 1 & 0 & -6.296472 & -1.732851 & 2.138251 \\
\hline 59 & 1 & 0 & -4.965897 & -4.087232 & -1.203258 \\
\hline 60 & 1 & 0 & -6.627795 & -3.646195 & 0.592245 \\
\hline
\end{tabular}




$\begin{array}{rrrrrr}61 & 6 & 0 & -2.137025 & 0.901164 & 1.334480 \\ 62 & 6 & 0 & -2.858396 & 2.097683 & 1.312555 \\ 63 & 6 & 0 & -1.402384 & 0.562835 & 2.475918 \\ 64 & 6 & 0 & -2.834611 & 2.946470 & 2.415320 \\ 65 & 1 & 0 & -3.423807 & 2.377166 & 0.422993 \\ 66 & 6 & 0 & -1.379323 & 1.412387 & 3.576814 \\ 67 & 1 & 0 & -0.824653 & -0.363778 & 2.488129 \\ 68 & 6 & 0 & -2.093088 & 2.607424 & 3.544446 \\ 69 & 1 & 0 & -3.394852 & 3.880177 & 2.390898 \\ 70 & 1 & 0 & -0.795395 & 1.143190 & 4.455390 \\ 71 & 1 & 0 & -2.070379 & 3.278319 & 4.401663\end{array}$

Zero-point correction= 0.558759 (Hartree/Particle)

Thermal correction to Energy $=\quad 0.596176$

Thermal correction to Enthalpy $=\quad 0.597120$

Thermal correction to Gibbs Free Energy= $\quad 0.484894$

$\begin{array}{lr}\text { Sum of electronic and zero-point Energies }= & -2209.649481 \\ \text { Sum of electronic and thermal Energies }= & -2209.612064\end{array}$

$\begin{array}{ll}\text { Sum of electronic and thermal Energies }= & -2209.612064 \\ \text { Sum of electronic and thermal Enthalpies }= & -2209.611120\end{array}$

$\begin{array}{ll}\text { Sum of electronic and thermal Enthalpies }= & -2209.611120 \\ \text { Sum of electronic and thermal Free Energies }= & -2209.723345\end{array}$

M06 /6-311++G(d,p)-SDD/SMD//M06/6-31G(d)-LANL2DZ energy in toluene solvent $=-2211.8614965$

INT7'

\begin{tabular}{|c|c|c|c|c|c|}
\hline \multirow{2}{*}{$\begin{array}{l}\text { Center } \\
\text { Number }\end{array}$} & \multirow{2}{*}{$\begin{array}{l}\text { Atomic } \\
\text { Number }\end{array}$} & \multirow{2}{*}{$\begin{array}{l}\text { Atomic } \\
\text { Type }\end{array}$} & \multicolumn{3}{|c|}{ Coordinates (Angstroms) } \\
\hline & & & $\mathrm{X}$ & $Y$ & \\
\hline 1 & 6 & 0 & -2.408446 & 4.465972 & 0.843414 \\
\hline 2 & 6 & 0 & -2.506787 & 4.021445 & -0.472557 \\
\hline 3 & 6 & 0 & -1.815430 & 2.879869 & -0.882164 \\
\hline 4 & 6 & 0 & -1.020701 & 2.172862 & 0.021788 \\
\hline 5 & 6 & 0 & -0.941563 & 2.612491 & 1.344359 \\
\hline 6 & 6 & 0 & -1.621251 & 3.759477 & 1.750448 \\
\hline 7 & 1 & 0 & -3.122950 & 4.565501 & -1.188813 \\
\hline 8 & 1 & 0 & -1.895132 & 2.546646 & -1.919000 \\
\hline 9 & 1 & 0 & -0.347716 & 2.058092 & 2.074358 \\
\hline 10 & 1 & 0 & -1.544118 & 4.095654 & 2.784474 \\
\hline 11 & 53 & 0 & 1.413257 & -1.550065 & -1.837804 \\
\hline 12 & 46 & 0 & 0.048045 & 0.586387 & -0.664593 \\
\hline 13 & 15 & 0 & -1.609733 & -0.806652 & 0.185865 \\
\hline 14 & 6 & 0 & 2.170505 & 3.166547 & -0.006049 \\
\hline 15 & 1 & 0 & 2.570276 & 3.440305 & 0.963797 \\
\hline 16 & 6 & 0 & 2.219145 & 1.888554 & -0.633436 \\
\hline 17 & 6 & 0 & 1.518322 & 2.024663 & -1.822374 \\
\hline 18 & 1 & 0 & 2.773924 & 1.003457 & -0.310320 \\
\hline 19 & 6 & 0 & 1.473670 & 3.987862 & -0.841287 \\
\hline 20 & 8 & 0 & 1.095840 & 3.320899 & -1.955952 \\
\hline 21 & 6 & 0 & -2.671604 & -1.572570 & -1.086177 \\
\hline 22 & 6 & 0 & -2.363755 & -1.431670 & -2.440320 \\
\hline 23 & 6 & 0 & -3.820482 & -2.280164 & -0.709020 \\
\hline 24 & 6 & 0 & -3.191744 & -1.996962 & -3.406179 \\
\hline 25 & 1 & 0 & -1.461052 & -0.893180 & -2.730741 \\
\hline 26 & 6 & 0 & -4.638493 & -2.850583 & -1.675286 \\
\hline 27 & 1 & 0 & -4.077456 & -2.375349 & 0.346751 \\
\hline 28 & 6 & 0 & -4.324548 & -2.706881 & -3.025419 \\
\hline 29 & 1 & 0 & -2.944167 & -1.883450 & -4.459939 \\
\hline 30 & 1 & 0 & -5.526936 & -3.403982 & -1.375777 \\
\hline 31 & 1 & 0 & -4.970420 & -3.148885 & -3.782518 \\
\hline 32 & 6 & 0 & -0.837987 & -2.158298 & 1.139579 \\
\hline 33 & 6 & 0 & 0.087851 & -1.811144 & 2.129811 \\
\hline 34 & 6 & 0 & -1.088696 & -3.505602 & 0.879549 \\
\hline 35 & 6 & 0 & 0.732759 & -2.796848 & 2.866436 \\
\hline 36 & 1 & 0 & 0.320515 & -0.758374 & 2.311807 \\
\hline 37 & 6 & 0 & -0.426128 & -4.490528 & 1.605956 \\
\hline 38 & 1 & 0 & -1.783348 & -3.790307 & 0.090547 \\
\hline 39 & 6 & 0 & 0.481028 & -4.140183 & 2.599879 \\
\hline 40 & 1 & 0 & 1.451887 & -2.514846 & 3.635952 \\
\hline 41 & 1 & 0 & -0.616232 & -5.539522 & 1.385523 \\
\hline 42 & 1 & 0 & 0.999757 & -4.914394 & 3.162904 \\
\hline 43 & 6 & 0 & -2.852832 & -0.089913 & 1.329863 \\
\hline 44 & 6 & 0 & -3.827991 & 0.757594 & 0.790471 \\
\hline 45 & 6 & 0 & -2.832569 & -0.311180 & 2.707225 \\
\hline 46 & 6 & 0 & -4.756441 & 1.376614 & 1.614388 \\
\hline 47 & 1 & 0 & -3.849857 & 0.947879 & -0.282686 \\
\hline 48 & 6 & 0 & -3.763938 & 0.314774 & 3.532885 \\
\hline 49 & 1 & 0 & -2.093919 & -0.980966 & 3.144561 \\
\hline 50 & 6 & 0 & -4.722787 & 1.160883 & 2.990743 \\
\hline 51 & 1 & 0 & -5.501606 & 2.041762 & 1.181246 \\
\hline 52 & 1 & 0 & -3.738409 & 0.131891 & 4.606000 \\
\hline 53 & 1 & 0 & -5.448145 & 1.650906 & 3.638174 \\
\hline 54 & 6 & 0 & 1.024056 & 5.389639 & -0.723014 \\
\hline 55 & 1 & 0 & 1.480027 & 5.860327 & 0.154055 \\
\hline 56 & 1 & 0 & -0.068656 & 5.431859 & -0.608003 \\
\hline 57 & 1 & 0 & 1.523630 & 1.434696 & -2.732187 \\
\hline 58 & 1 & 0 & 1.290615 & 5.971029 & -1.614754 \\
\hline 59 & 1 & 0 & -2.949676 & 5.355552 & 1.163364 \\
\hline 60 & 7 & 0 & 4.243891 & -0.347145 & 0.852679 \\
\hline 61 & 6 & 0 & 3.716326 & -1.641044 & 1.274148 \\
\hline 62 & 1 & 0 & 3.578758 & -2.242842 & 0.365331 \\
\hline 63 & 6 & 0 & 5.446330 & -0.456112 & 0.033796 \\
\hline 64 & 1 & 0 & 5.386525 & -1.406706 & -0.515652 \\
\hline 65 & 1 & 0 & 6.360484 & -0.514325 & 0.662779 \\
\hline 66 & 6 & 0 & 4.410142 & 0.608056 & 1.936185 \\
\hline
\end{tabular}




$\begin{array}{rrrrrr}67 & 1 & 0 & 4.748550 & 1.557106 & 1.491613 \\ 68 & 1 & 0 & 5.219431 & 0.302910 & 2.635684 \\ 69 & 1 & 0 & 2.698927 & -1.488467 & 1.659870 \\ 70 & 6 & 0 & 3.130184 & 0.859646 & 2.715241 \\ 71 & 1 & 0 & 2.849829 & 0.002650 & 3.343222 \\ 72 & 1 & 0 & 3.246988 & 1.725165 & 3.379758 \\ 73 & 1 & 0 & 2.293548 & 1.059806 & 2.028625 \\ 74 & 6 & 0 & 5.577511 & 0.672360 & -0.974127 \\ 75 & 1 & 0 & 5.582525 & 1.661995 & -0.496948 \\ 76 & 1 & 0 & 6.512976 & 0.577356 & -1.540181 \\ 77 & 1 & 0 & 4.740747 & 0.642969 & -1.684093 \\ 78 & 6 & 0 & 4.545852 & -2.412276 & 2.294014 \\ 79 & 1 & 0 & 4.628970 & -1.882275 & 3.253865 \\ 80 & 1 & 0 & 4.077631 & -3.385448 & 2.494620 \\ 81 & 1 & 0 & 5.565521 & -2.604008 & 1.931946\end{array}$

Zero-point correction=

0.671791 (Hartree/Particle)

Thermal correction to Energy=

Thermal correction to Gibbs Free Energy=

Sum of electronic and zero-point Energies

Sum of electronic and thermal Energies=

Sum of electronic and thermal Enthalpies=

0.715462

0.593330

$-1966.019058$

$-1965.976332$

$-1965.975387$

M06 /6-311++G(d,p)-SDD/SMD//M06/6-31G(d)-LANL2DZ energy in toluene solvent = -1968.3542822

INT8

\begin{tabular}{|c|c|c|c|c|c|}
\hline \multirow{2}{*}{$\begin{array}{l}\text { Center } \\
\text { Number }\end{array}$} & \multirow{2}{*}{$\begin{array}{l}\text { Atomic } \\
\text { Number }\end{array}$} & \multirow{2}{*}{$\begin{array}{l}\text { Atomic } \\
\text { Type }\end{array}$} & \multicolumn{3}{|c|}{ Coordinates (Angstroms) } \\
\hline & & & $X$ & $\mathrm{Y}$ & \\
\hline 1 & 53 & 0 & -0.010701 & -3.443229 & -0.106801 \\
\hline 2 & 15 & 0 & 2.333212 & 0.400512 & -0.065253 \\
\hline 3 & 46 & 0 & 0.002460 & 0.137289 & -0.198730 \\
\hline 4 & 15 & 0 & -2.328098 & 0.407635 & -0.049900 \\
\hline 5 & 1 & 0 & 0.000929 & -1.603195 & -0.142736 \\
\hline 6 & 6 & 0 & 2.781066 & 2.140069 & -0.433380 \\
\hline 7 & 6 & 0 & 1.914461 & 3.134357 & 0.036153 \\
\hline 8 & 6 & 0 & 3.917331 & 2.511615 & -1.154832 \\
\hline 9 & 6 & 0 & 2.183839 & 4.475994 & -0.203417 \\
\hline 10 & 1 & 0 & 1.014795 & 2.845520 & 0.585741 \\
\hline 11 & 6 & 0 & 4.181828 & 3.856604 & -1.400464 \\
\hline 12 & 1 & 0 & 4.595064 & 1.745997 & -1.533048 \\
\hline 13 & 6 & 0 & 3.318396 & 4.838617 & -0.925830 \\
\hline 14 & 1 & 0 & 1.499799 & 5.238355 & 0.167718 \\
\hline 15 & 1 & 0 & 5.066899 & 4.137003 & -1.969636 \\
\hline 16 & 1 & 0 & 3.526854 & 5.888905 & -1.124093 \\
\hline 17 & 6 & 0 & 3.399401 & -0.592028 & -1.168250 \\
\hline 18 & 6 & 0 & 4.727257 & -0.906558 & -0.864286 \\
\hline 19 & 6 & 0 & 2.846725 & -1.035100 & -2.373120 \\
\hline 20 & 6 & 0 & 5.493166 & -1.642308 & -1.761913 \\
\hline 21 & 1 & 0 & 5.161583 & -0.577913 & 0.080877 \\
\hline 22 & 6 & 0 & 3.615086 & -1.768323 & -3.270193 \\
\hline 23 & 1 & 0 & 1.801461 & -0.811888 & -2.594996 \\
\hline 24 & 6 & 0 & 4.938269 & -2.071381 & -2.964826 \\
\hline 25 & 1 & 0 & 6.525966 & -1.887341 & -1.518841 \\
\hline 26 & 1 & 0 & 3.175233 & -2.116123 & -4.203169 \\
\hline 27 & 1 & 0 & 5.538154 & -2.654589 & -3.661757 \\
\hline 28 & 6 & 0 & 3.037145 & 0.115286 & 1.600916 \\
\hline 29 & 6 & 0 & 2.594820 & -1.010975 & 2.304210 \\
\hline 30 & 6 & 0 & 3.992407 & 0.954148 & 2.180619 \\
\hline 31 & 6 & 0 & 3.112203 & -1.298752 & 3.561621 \\
\hline 32 & 1 & 0 & 1.843438 & -1.667696 & 1.859490 \\
\hline 33 & 6 & 0 & 4.500731 & 0.667814 & 3.444441 \\
\hline 34 & 1 & 0 & 4.339483 & 1.837290 & 1.643747 \\
\hline 35 & 6 & 0 & 4.063310 & -0.458046 & 4.134401 \\
\hline 36 & 1 & 0 & 2.766629 & -2.180645 & 4.098313 \\
\hline 37 & 1 & 0 & 5.241840 & 1.329351 & 3.890592 \\
\hline 38 & 1 & 0 & 4.462201 & -0.679442 & 5.123165 \\
\hline 39 & 6 & 0 & -2.688888 & 2.181720 & 0.243692 \\
\hline 40 & 6 & 0 & -1.949013 & 3.113581 & -0.494564 \\
\hline 41 & 6 & 0 & -3.641380 & 2.639655 & 1.156327 \\
\hline 42 & 6 & 0 & -2.163280 & 4.476470 & -0.330317 \\
\hline 43 & 1 & 0 & -1.194741 & 2.757809 & -1.200660 \\
\hline 44 & 6 & 0 & -3.848711 & 4.006154 & 1.326462 \\
\hline 45 & 1 & 0 & -4.219543 & 1.926029 & 1.743250 \\
\hline 46 & 6 & 0 & -3.113346 & 4.924883 & 0.585000 \\
\hline 47 & 1 & 0 & -1.581651 & 5.189324 & -0.913278 \\
\hline 48 & 1 & 0 & -4.589198 & 4.354161 & 2.045094 \\
\hline 49 & 1 & 0 & -3.277001 & 5.992554 & 0.722893 \\
\hline 50 & 6 & 0 & -3.130316 & -0.468641 & 1.338905 \\
\hline 51 & 6 & 0 & -4.506874 & -0.713686 & 1.378392 \\
\hline 52 & 6 & 0 & -2.326021 & -0.892441 & 2.400194 \\
\hline 53 & 6 & 0 & -5.070864 & -1.357774 & 2.473428 \\
\hline 54 & 1 & 0 & -5.137430 & -0.402967 & 0.543877 \\
\hline 55 & 6 & 0 & -2.892773 & -1.534852 & 3.495735 \\
\hline 56 & 1 & 0 & -1.246621 & -0.730220 & 2.348314 \\
\hline 57 & 6 & 0 & -4.264207 & -1.765525 & 3.533153 \\
\hline 58 & 1 & 0 & -6.142623 & -1.548376 & 2.497976 \\
\hline 59 & 1 & 0 & -2.259429 & -1.866797 & 4.316442 \\
\hline 60 & 1 & 0 & -4.707402 & -2.275397 & 4.387217 \\
\hline 61 & 6 & 0 & -3.382614 & -0.015165 & -1.485964 \\
\hline 62 & 6 & 0 & -3.220430 & -1.285607 & -2.050765 \\
\hline
\end{tabular}




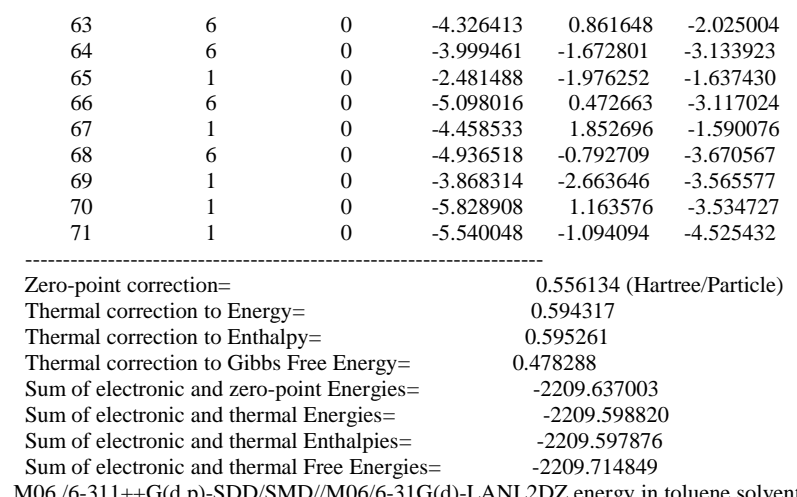

M06 /6-311++G(d,p)-SDD/SMD//M06/6-31G(d)-LANL2DZ energy in toluene solvent $=-2211.8442401$

\section{INT9}

\begin{tabular}{|c|c|c|c|c|c|}
\hline \multirow{2}{*}{$\begin{array}{l}\text { Center } \\
\text { Number }\end{array}$} & \multirow{2}{*}{$\begin{array}{l}\text { Atomic } \\
\text { Number }\end{array}$} & \multirow{2}{*}{$\begin{array}{l}\text { Atomic } \\
\text { Type }\end{array}$} & \multicolumn{3}{|c|}{ Coordinates (Angstroms) } \\
\hline & & & $\mathrm{X}$ & $\mathrm{Y}$ & \\
\hline 1 & 6 & 0 & 0.766764 & 4.875987 & -0.061317 \\
\hline 2 & 6 & 0 & 0.443638 & 4.138510 & -1.197128 \\
\hline 3 & 6 & 0 & 0.524076 & 2.747307 & -1.178044 \\
\hline 4 & 6 & 0 & 0.910919 & 2.068438 & -0.018503 \\
\hline 5 & 6 & 0 & 1.255688 & 2.818879 & 1.108884 \\
\hline 6 & 6 & 0 & 1.175952 & 4.211600 & 1.091327 \\
\hline 7 & 1 & 0 & 0.118363 & 4.647709 & -2.104687 \\
\hline 8 & 1 & 0 & 0.242330 & 2.186583 & -2.072977 \\
\hline 9 & 1 & 0 & 1.580333 & 2.314759 & 2.021545 \\
\hline 10 & 1 & 0 & 1.438458 & 4.779315 & 1.984699 \\
\hline 11 & 53 & 0 & 1.450737 & -2.712296 & -0.079500 \\
\hline 12 & 46 & 0 & 0.959977 & 0.017095 & 0.012287 \\
\hline 13 & 15 & 0 & -1.438091 & -0.114583 & 0.082087 \\
\hline 14 & 6 & 0 & 3.924063 & 0.280438 & 0.917035 \\
\hline 15 & 1 & 0 & 3.775406 & 0.245295 & 1.991138 \\
\hline 16 & 6 & 0 & 2.942634 & 0.217707 & -0.069614 \\
\hline 17 & 6 & 0 & 3.676772 & 0.295997 & -1.345119 \\
\hline 18 & 1 & 0 & 3.533808 & -0.594030 & -1.970096 \\
\hline 19 & 6 & 0 & 5.160982 & 0.365420 & 0.296462 \\
\hline 20 & 8 & 0 & 5.093298 & 0.380675 & -1.018519 \\
\hline 21 & 6 & 0 & -2.301771 & 1.477353 & -0.202635 \\
\hline 22 & 6 & 0 & -2.982941 & 1.754106 & -1.388513 \\
\hline 23 & 6 & 0 & -2.168246 & 2.487093 & 0.757607 \\
\hline 24 & 6 & 0 & -3.519925 & 3.020451 & -1.611047 \\
\hline 25 & 1 & 0 & -3.095819 & 0.980571 & -2.147711 \\
\hline 26 & 6 & 0 & -2.707361 & 3.746008 & 0.536065 \\
\hline 27 & 1 & 0 & -1.617641 & 2.288934 & 1.678103 \\
\hline 28 & 6 & 0 & -3.382893 & 4.016726 & -0.652480 \\
\hline 29 & 1 & 0 & -4.050081 & 3.224350 & -2.540308 \\
\hline 30 & 1 & 0 & -2.586888 & 4.523662 & 1.288663 \\
\hline 31 & 1 & 0 & -3.800964 & 5.006721 & -0.828912 \\
\hline 32 & 6 & 0 & -2.149844 & -1.195438 & -1.210023 \\
\hline 33 & 6 & 0 & -3.399481 & -1.803752 & -1.076534 \\
\hline 34 & 6 & 0 & -1.439188 & -1.339885 & -2.404326 \\
\hline 35 & 6 & 0 & -3.932877 & -2.542284 & -2.127401 \\
\hline 36 & 1 & 0 & -3.956390 & -1.704443 & -0.144706 \\
\hline 37 & 6 & 0 & -1.977807 & -2.070710 & -3.457348 \\
\hline 38 & 1 & 0 & -0.451166 & -0.885064 & -2.497108 \\
\hline 39 & 6 & 0 & -3.224806 & -2.672665 & -3.318530 \\
\hline 40 & 1 & 0 & -4.905310 & -3.019101 & -2.014402 \\
\hline 41 & 1 & 0 & -1.416886 & -2.180897 & -4.383785 \\
\hline 42 & 1 & 0 & -3.643925 & -3.252055 & -4.139763 \\
\hline 43 & 6 & 0 & -2.155505 & -0.702077 & 1.660962 \\
\hline 44 & 6 & 0 & -3.436870 & -0.323230 & 2.079051 \\
\hline 45 & 6 & 0 & -1.401691 & -1.565351 & 2.461311 \\
\hline 46 & 6 & 0 & -3.954772 & -0.804720 & 3.276131 \\
\hline 47 & 1 & 0 & -4.027361 & 0.361047 & 1.469141 \\
\hline 48 & 6 & 0 & -1.924513 & -2.045541 & 3.658088 \\
\hline 49 & 1 & 0 & -0.404632 & -1.867148 & 2.136835 \\
\hline 50 & 6 & 0 & -3.198172 & -1.665156 & 4.067169 \\
\hline 51 & 1 & 0 & -4.951505 & -0.502758 & 3.594295 \\
\hline 52 & 1 & 0 & -1.328453 & -2.717140 & 4.273593 \\
\hline 53 & 1 & 0 & -3.603389 & -2.037962 & 5.006838 \\
\hline 54 & 6 & 0 & 6.511922 & 0.432624 & 0.888215 \\
\hline 55 & 1 & 0 & 7.095231 & -0.446132 & 0.584617 \\
\hline 56 & 1 & 0 & 6.461501 & 0.467920 & 1.979736 \\
\hline 57 & 1 & 0 & 0.702210 & 5.963284 & -0.075714 \\
\hline 58 & 1 & 0 & 7.044203 & 1.317394 & 0.518048 \\
\hline 59 & 1 & 0 & 3.417089 & 1.194808 & -1.920392 \\
\hline
\end{tabular}

Zero-point correction= 0.464252 (Hartree/Particle) Thermal correction to Energy= Thermal correction to Enthalpy=

Thermal correction to Gibbs Free Energy= Sum of electronic and zero-point Energies= Sum of electronic and thermal Energies= Sum of electronic and thermal Enthalpies= Sum of electronic and thermal Free Energies= 0.496598 0.497542 0.395766 $-1674.010219$ -1674.010219
-1673.977872 -1673.977872
-1673.976928 $-1674.078705$ 
M06 /6-311++G(d,p)-SDD/SMD//M06/6-31G(d)-LANL2DZ energy in toluene solvent $=-1676.0489528$

TSO

\begin{tabular}{|c|c|c|c|c|c|}
\hline \multirow{2}{*}{$\begin{array}{l}\text { Center } \\
\text { Number }\end{array}$} & \multirow{2}{*}{$\begin{array}{l}\text { Atomic } \\
\text { Number }\end{array}$} & \multirow{2}{*}{$\begin{array}{l}\text { Atomic } \\
\text { Type }\end{array}$} & \multicolumn{3}{|c|}{ Coordinates (Angstroms) } \\
\hline & & & $\mathrm{X}$ & $\mathrm{Y}$ & 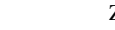 \\
\hline 1 & 46 & 0 & 0.194139 & -0.774393 & -0.052641 \\
\hline 2 & 15 & 0 & 2.154529 & 0.442255 & 0.303496 \\
\hline 3 & 15 & 0 & -2.182885 & 0.332379 & -0.097460 \\
\hline 4 & 53 & 0 & -0.461849 & -3.364242 & 0.632757 \\
\hline 5 & 6 & 0 & 0.794558 & -0.926962 & -1.958684 \\
\hline 6 & 6 & 0 & 0.191024 & -0.107218 & -2.907399 \\
\hline 7 & 6 & 0 & 1.733305 & -1.885331 & -2.327398 \\
\hline 8 & 6 & 0 & 0.524893 & -0.266994 & -4.253647 \\
\hline 9 & 1 & 0 & -0.522864 & 0.663629 & -2.617563 \\
\hline 10 & 6 & 0 & 2.061385 & -2.025447 & -3.676251 \\
\hline 11 & 1 & 0 & 2.198601 & -2.527991 & -1.582926 \\
\hline 12 & 6 & 0 & 1.461919 & -1.219468 & -4.639811 \\
\hline 13 & 1 & 0 & 0.048571 & 0.371239 & -4.997180 \\
\hline 14 & 1 & 0 & 2.789648 & -2.781082 & -3.968423 \\
\hline 15 & 1 & 0 & 1.722718 & -1.336763 & -5.690382 \\
\hline 16 & 6 & 0 & -2.381702 & 1.933533 & -0.979674 \\
\hline 17 & 6 & 0 & -3.596822 & 2.383502 & -1.503868 \\
\hline 18 & 6 & 0 & -1.248080 & 2.745207 & -1.105603 \\
\hline 19 & 6 & 0 & -3.675738 & 3.622080 & -2.132774 \\
\hline 20 & 1 & 0 & -4.486264 & 1.758817 & -1.422166 \\
\hline 21 & 6 & 0 & -1.328161 & 3.986232 & -1.727932 \\
\hline 22 & 1 & 0 & -0.287642 & 2.392430 & -0.720962 \\
\hline 23 & 6 & 0 & -2.544115 & 4.425866 & -2.242984 \\
\hline 24 & 1 & 0 & -4.627289 & 3.962138 & -2.538966 \\
\hline 25 & 1 & 0 & -0.432693 & 4.600117 & -1.817566 \\
\hline 26 & 1 & 0 & -2.610080 & 5.393818 & -2.737678 \\
\hline 27 & 6 & 0 & -2.644261 & 0.767573 & 1.627325 \\
\hline 28 & 6 & 0 & -2.917099 & 2.071512 & 2.050076 \\
\hline 29 & 6 & 0 & -2.648667 & -0.271621 & 2.569387 \\
\hline 30 & 6 & 0 & -3.198971 & 2.330984 & 3.390485 \\
\hline 31 & 1 & 0 & -2.917215 & 2.889505 & 1.329004 \\
\hline 32 & 6 & 0 & -2.939014 & -0.009046 & 3.902004 \\
\hline 33 & 1 & 0 & -2.421324 & -1.292843 & 2.253228 \\
\hline 34 & 6 & 0 & -3.214640 & 1.292909 & 4.316720 \\
\hline 35 & 1 & 0 & -3.418694 & 3.350101 & 3.706504 \\
\hline 36 & 1 & 0 & -2.944714 & -0.825816 & 4.621698 \\
\hline 37 & 1 & 0 & -3.441746 & 1.497257 & 5.362148 \\
\hline 38 & 6 & 0 & -3.613052 & -0.659743 & -0.660487 \\
\hline 39 & 6 & 0 & -3.391134 & -1.588177 & -1.681308 \\
\hline 40 & 6 & 0 & -4.897721 & -0.522772 & -0.124182 \\
\hline 41 & 6 & 0 & -4.443056 & -2.354189 & -2.172688 \\
\hline 42 & 1 & 0 & -2.384282 & -1.726473 & -2.077604 \\
\hline 43 & 6 & 0 & -5.946357 & -1.293670 & -0.613660 \\
\hline 44 & 1 & 0 & -5.074531 & 0.188358 & 0.684219 \\
\hline 45 & 6 & 0 & -5.719443 & -2.207774 & -1.639261 \\
\hline 46 & 1 & 0 & -4.259539 & -3.081378 & -2.961554 \\
\hline 47 & 1 & 0 & -6.943229 & -1.184060 & -0.188971 \\
\hline 48 & 1 & 0 & -6.540284 & -2.816226 & -2.015929 \\
\hline 49 & 6 & 0 & 2.016052 & 0.920127 & 2.062080 \\
\hline 50 & 6 & 0 & 3.062131 & 0.750810 & 2.974051 \\
\hline 51 & 6 & 0 & 0.789888 & 1.426258 & 2.511650 \\
\hline 52 & 6 & 0 & 2.877931 & 1.081543 & 4.312444 \\
\hline 53 & 1 & 0 & 4.019592 & 0.354604 & 2.637328 \\
\hline 54 & 6 & 0 & 0.609669 & 1.757535 & 3.849000 \\
\hline 55 & 1 & 0 & -0.033835 & 1.564055 & 1.808368 \\
\hline 56 & 6 & 0 & 1.654533 & 1.579947 & 4.751331 \\
\hline 57 & 1 & 0 & 3.695139 & 0.944538 & 5.018644 \\
\hline 58 & 1 & 0 & -0.355594 & 2.138614 & 4.180595 \\
\hline 59 & 1 & 0 & 1.514295 & 1.827385 & 5.802324 \\
\hline 60 & 6 & 0 & 2.458601 & 1.994004 & -0.611256 \\
\hline 61 & 6 & 0 & 2.669365 & 1.930541 & -1.996380 \\
\hline 62 & 6 & 0 & 2.384149 & 3.244248 & 0.010205 \\
\hline 63 & 6 & 0 & 2.799258 & 3.097153 & -2.739120 \\
\hline 64 & 1 & 0 & 2.732346 & 0.962947 & -2.496080 \\
\hline 65 & 6 & 0 & 2.515627 & 4.410295 & -0.739240 \\
\hline 66 & 1 & 0 & 2.220490 & 3.309571 & 1.085300 \\
\hline 67 & 6 & 0 & 2.720264 & 4.339721 & -2.113479 \\
\hline 68 & 1 & 0 & 2.960561 & 3.033392 & -3.813905 \\
\hline 69 & 1 & 0 & 2.459950 & 5.378042 & -0.243065 \\
\hline 70 & 1 & 0 & 2.820286 & 5.252648 & -2.698448 \\
\hline 71 & 6 & 0 & 3.716452 & -0.492665 & 0.213398 \\
\hline 72 & 6 & 0 & 3.693444 & -1.825726 & 0.641459 \\
\hline 73 & 6 & 0 & 4.920496 & 0.080062 & -0.202684 \\
\hline 74 & 6 & 0 & 4.865836 & -2.571573 & 0.655986 \\
\hline 75 & 1 & 0 & 2.748798 & -2.274518 & 0.961345 \\
\hline 76 & 6 & 0 & 6.089345 & -0.674877 & -0.195052 \\
\hline 77 & 1 & 0 & 4.946761 & 1.118942 & -0.530485 \\
\hline 78 & 6 & 0 & 6.063218 & -1.998144 & 0.233816 \\
\hline 79 & 1 & 0 & 4.840989 & -3.606968 & 0.990583 \\
\hline 80 & 1 & 0 & 7.024896 & -0.225342 & -0.524097 \\
\hline 81 & 1 & 0 & 6.979262 & -2.586771 & 0.237964 \\
\hline
\end{tabular}

Zero-point correction=

0.638144 (Hartree/Particle)

Thermal correction to Energy=

Thermal correction to Enthalpy=

0.680905

Thermal correction to Gibbs Free Energy= 
$\begin{array}{ll}\text { Sum of electronic and zero-point Energies }= & -2440.405269 \\ \text { Sum of electronic and thermal Energies }= & -2440.362508\end{array}$

$\begin{array}{ll}\text { Sum of electronic and thermal Enthalpies }= & -2440.361564 \\ \text { Sum of electronic and thermal Free Energies }= & -2440.486471\end{array}$

M06 /6-311++G(d,p)-SDD/SMD//M06/6-31G(d)-LANL2DZ energy in toluene solvent $=-2442.7600627$

TS1

\begin{tabular}{|c|c|c|c|c|c|}
\hline \multirow{2}{*}{$\begin{array}{l}\text { Center } \\
\text { Number }\end{array}$} & \multirow{2}{*}{$\begin{array}{l}\text { Atomic } \\
\text { Number }\end{array}$} & \multirow{2}{*}{$\begin{array}{l}\text { Atomic } \\
\text { Type }\end{array}$} & \multicolumn{3}{|c|}{ Coordinates (Angstroms) } \\
\hline & & & $\mathrm{X}$ & $\mathrm{Y}$ & \\
\hline 1 & 6 & 0 & 3.051575 & -3.171767 & -2.602336 \\
\hline 2 & 6 & 0 & 3.506691 & -2.938276 & -1.300706 \\
\hline 3 & 6 & 0 & 2.613474 & -2.678925 & -0.274229 \\
\hline 4 & 6 & 0 & 1.239906 & -2.621696 & -0.554656 \\
\hline 5 & 6 & 0 & 0.772127 & -2.909127 & -1.844207 \\
\hline 6 & 6 & 0 & 1.690621 & -3.150799 & -2.869675 \\
\hline 7 & 1 & 0 & 4.575418 & -2.939407 & -1.089193 \\
\hline 8 & 1 & 0 & 2.972368 & -2.493051 & 0.738103 \\
\hline 9 & 1 & 0 & -0.297584 & -2.972886 & -2.036199 \\
\hline 10 & 1 & 0 & 1.323924 & -3.357005 & -3.874765 \\
\hline 11 & 53 & 0 & -0.220248 & -3.285555 & 1.170609 \\
\hline 12 & 15 & 0 & 1.802233 & 0.847587 & 0.129116 \\
\hline 13 & 46 & 0 & 0.056802 & -0.835356 & -0.175334 \\
\hline 14 & 15 & 0 & -2.133060 & 0.209883 & -0.253489 \\
\hline 15 & 6 & 0 & 3.239681 & 0.607691 & -0.986651 \\
\hline 16 & 6 & 0 & 2.953848 & 0.394979 & -2.341252 \\
\hline 17 & 6 & 0 & 4.568971 & 0.566022 & -0.562238 \\
\hline 18 & 6 & 0 & 3.976932 & 0.177954 & -3.255103 \\
\hline 19 & 1 & 0 & 1.912244 & 0.378561 & -2.672257 \\
\hline 20 & 6 & 0 & 5.592968 & 0.330965 & -1.476427 \\
\hline 21 & 1 & 0 & 4.809630 & 0.711749 & 0.490348 \\
\hline 22 & 6 & 0 & 5.300890 & 0.143040 & -2.823105 \\
\hline 23 & 1 & 0 & 3.738737 & 0.011544 & -4.304812 \\
\hline 24 & 1 & 0 & 6.625703 & 0.296980 & -1.131902 \\
\hline 25 & 1 & 0 & 6.103068 & -0.038249 & -3.536975 \\
\hline 26 & 6 & 0 & -2.603403 & 1.323611 & -1.636472 \\
\hline 27 & 6 & 0 & -3.909892 & 1.427938 & -2.124066 \\
\hline 28 & 6 & 0 & -1.602645 & 2.125532 & -2.195576 \\
\hline 29 & 6 & 0 & -4.207434 & 2.319423 & -3.150051 \\
\hline 30 & 1 & 0 & -4.699302 & 0.808527 & -1.697412 \\
\hline 31 & 6 & 0 & -1.904309 & 3.024496 & -3.212083 \\
\hline 32 & 1 & 0 & -0.579461 & 2.045147 & -1.823927 \\
\hline 33 & 6 & 0 & -3.206931 & 3.120316 & -3.693345 \\
\hline 34 & 1 & 0 & -5.227193 & 2.389843 & -3.526067 \\
\hline 35 & 1 & 0 & -1.115379 & 3.645758 & -3.633388 \\
\hline 36 & 1 & 0 & -3.442649 & 3.817576 & -4.496046 \\
\hline 37 & 6 & 0 & 2.540395 & 0.938019 & 1.809329 \\
\hline 38 & 6 & 0 & 3.337185 & 2.011820 & 2.224749 \\
\hline 39 & 6 & 0 & 2.280545 & -0.101258 & 2.705101 \\
\hline 40 & 6 & 0 & 3.873970 & 2.034725 & 3.506458 \\
\hline 41 & 1 & 0 & 3.531277 & 2.836482 & 1.537358 \\
\hline 42 & 6 & 0 & 2.819989 & -0.079647 & 3.988825 \\
\hline 43 & 1 & 0 & 1.638387 & -0.927026 & 2.389776 \\
\hline 44 & 6 & 0 & 3.616913 & 0.987341 & 4.388840 \\
\hline 45 & 1 & 0 & 4.491134 & 2.875197 & 3.820745 \\
\hline 46 & 1 & 0 & 2.609525 & -0.895552 & 4.678389 \\
\hline 47 & 1 & 0 & 4.035068 & 1.008862 & 5.394182 \\
\hline 48 & 6 & 0 & 1.365894 & 2.615016 & -0.157980 \\
\hline 49 & 6 & 0 & 0.427379 & 3.194795 & 0.705835 \\
\hline 50 & 6 & 0 & 1.832682 & 3.361943 & -1.242947 \\
\hline 51 & 6 & 0 & -0.036087 & 4.485636 & 0.486085 \\
\hline 52 & 1 & 0 & 0.049764 & 2.624943 & 1.557295 \\
\hline 53 & 6 & 0 & 1.361435 & 4.654494 & -1.465045 \\
\hline 54 & 1 & 0 & 2.569071 & 2.935336 & -1.923536 \\
\hline 55 & 6 & 0 & 0.425293 & 5.218156 & -0.605306 \\
\hline 56 & 1 & 0 & -0.769470 & 4.913781 & 1.168379 \\
\hline 57 & 1 & 0 & 1.735223 & 5.223970 & -2.315249 \\
\hline 58 & 1 & 0 & 0.054838 & 6.226488 & -0.783387 \\
\hline 59 & 6 & 0 & -3.562395 & -0.942309 & -0.186580 \\
\hline 60 & 6 & 0 & -4.639711 & -0.797172 & 0.689868 \\
\hline 61 & 6 & 0 & -3.556847 & -2.017037 & -1.081780 \\
\hline 62 & 6 & 0 & -5.691428 & -1.710172 & 0.667736 \\
\hline 63 & 1 & 0 & -4.661218 & 0.035858 & 1.392310 \\
\hline 64 & 6 & 0 & -4.611619 & -2.919506 & -1.113334 \\
\hline 65 & 1 & 0 & -2.709516 & -2.140581 & -1.758847 \\
\hline 66 & 6 & 0 & -5.680996 & -2.769017 & -0.233454 \\
\hline 67 & 1 & 0 & -6.523748 & -1.590034 & 1.359776 \\
\hline 68 & 1 & 0 & -4.594711 & -3.750294 & -1.816898 \\
\hline 69 & 1 & 0 & -6.504051 & -3.481777 & -0.247906 \\
\hline 70 & 6 & 0 & -2.406439 & 1.261281 & 1.228056 \\
\hline 71 & 6 & 0 & -3.032182 & 2.508502 & 1.186348 \\
\hline 72 & 6 & 0 & -1.922027 & 0.780982 & 2.451164 \\
\hline 73 & 6 & 0 & -3.161868 & 3.267651 & 2.347295 \\
\hline 74 & 1 & 0 & -3.408760 & 2.896267 & 0.239399 \\
\hline 75 & 6 & 0 & -2.058457 & 1.536259 & 3.609929 \\
\hline 76 & 1 & 0 & -1.421599 & -0.190027 & 2.482600 \\
\hline 77 & 6 & 0 & -2.673343 & 2.786200 & 3.557565 \\
\hline 78 & 1 & 0 & -3.645940 & 4.242725 & 2.302699 \\
\hline 79 & 1 & 0 & -1.675478 & 1.153552 & 4.554699 \\
\hline 80 & 1 & 0 & -2.770152 & 3.384249 & 4.462419 \\
\hline 81 & 1 & 0 & 3.765413 & -3.377215 & -3.398471 \\
\hline
\end{tabular}


Zero-point correction $=$

Thermal correction to Energy=

Thermal correction to Enthalpy=

Thermal correction to Gibbs Free Energy=

Sum of electronic and zero-point Energies=

Sum of electronic and thermal Energies=

um of electronic and thermal Enthalpies $=$
0.639461 (Hartree/Particle)

0.681538

0.682482

0.561150

$-2440.39948$

$-2440.357404$

$-2440.356460$

$-2440.47779$

(d)

\begin{tabular}{|c|c|c|c|c|c|}
\hline \multirow{2}{*}{$\begin{array}{l}\text { Center } \\
\text { Number }\end{array}$} & \multirow{2}{*}{$\begin{array}{l}\text { Atomic } \\
\text { Number }\end{array}$} & \multirow{2}{*}{$\begin{array}{l}\text { Atomic } \\
\text { Type }\end{array}$} & \multicolumn{3}{|c|}{ Coordinates (Angstroms) } \\
\hline & & & $\mathrm{X}$ & $\mathrm{Y}$ & 2 \\
\hline 1 & 6 & 0 & 2.311198 & 3.806940 & -1.498252 \\
\hline 2 & 6 & 0 & 2.246902 & 2.754052 & -2.402565 \\
\hline 3 & 6 & 0 & 2.030897 & 1.452096 & -1.948060 \\
\hline 4 & 6 & 0 & 1.847953 & 1.204883 & -0.586110 \\
\hline 5 & 6 & 0 & 1.917715 & 2.266252 & 0.318762 \\
\hline 6 & 6 & 0 & 2.152335 & 3.558042 & -0.135398 \\
\hline 7 & 1 & 0 & 2.377635 & 2.936954 & -3.468479 \\
\hline 8 & 1 & 0 & 1.995343 & 0.634421 & -2.669576 \\
\hline 9 & 1 & 0 & 1.770162 & 2.088813 & 1.385408 \\
\hline 10 & 1 & 0 & 2.197758 & 4.379388 & 0.578784 \\
\hline 11 & 53 & 0 & -0.285275 & -3.084926 & -0.461259 \\
\hline 12 & 46 & 0 & 0.895295 & -0.634647 & -0.216813 \\
\hline 13 & 15 & 0 & -1.244780 & 0.386666 & 0.226949 \\
\hline 14 & 6 & 0 & 3.879947 & -0.144739 & 0.958958 \\
\hline 15 & 1 & 0 & 3.639086 & 0.644705 & 1.669560 \\
\hline 16 & 6 & 0 & 3.029155 & -0.526100 & 0.007389 \\
\hline 17 & 6 & 0 & 2.765695 & -1.543493 & -0.881518 \\
\hline 18 & 1 & 0 & 2.818841 & -2.575215 & -0.536341 \\
\hline 19 & 6 & 0 & 5.215079 & -0.789478 & 1.036044 \\
\hline 20 & 8 & 0 & 5.585676 & -1.641884 & 0.252051 \\
\hline 21 & 6 & 0 & -2.274146 & 0.138876 & -1.262869 \\
\hline 22 & 6 & 0 & -3.538955 & -0.449153 & -1.226315 \\
\hline 23 & 6 & 0 & -1.715995 & 0.482204 & -2.501256 \\
\hline 24 & 6 & 0 & -4.246117 & -0.660338 & -2.406138 \\
\hline 25 & 1 & 0 & -3.967501 & -0.767759 & -0.277685 \\
\hline 26 & 6 & 0 & -2.430258 & 0.281875 & -3.675343 \\
\hline 27 & 1 & 0 & -0.707153 & 0.897408 & -2.546503 \\
\hline 28 & 6 & 0 & -3.699259 & -0.289163 & -3.628892 \\
\hline 29 & 1 & 0 & -5.228690 & -1.127254 & -2.366057 \\
\hline 30 & 1 & 0 & -1.987563 & 0.556947 & -4.631090 \\
\hline 31 & 1 & 0 & -4.254534 & -0.458924 & -4.549766 \\
\hline 32 & 6 & 0 & -1.253642 & 2.201216 & 0.519185 \\
\hline 33 & 6 & 0 & -1.430236 & 3.130141 & -0.506691 \\
\hline 34 & 6 & 0 & -0.974183 & 2.662990 & 1.811740 \\
\hline 35 & 6 & 0 & -1.330732 & 4.493456 & -0.245896 \\
\hline 36 & 1 & 0 & -1.656879 & 2.795955 & -1.517832 \\
\hline 37 & 6 & 0 & -0.884873 & 4.024522 & 2.072228 \\
\hline 38 & 1 & 0 & -0.833754 & 1.947113 & 2.623514 \\
\hline 39 & 6 & 0 & -1.059807 & 4.944395 & 1.041087 \\
\hline 40 & 1 & 0 & -1.472441 & 5.207224 & -1.055817 \\
\hline 41 & 1 & 0 & -0.678824 & 4.368655 & 3.084642 \\
\hline 42 & 1 & 0 & -0.989309 & 6.011993 & 1.243373 \\
\hline 43 & 6 & 0 & -2.211031 & -0.209524 & 1.657861 \\
\hline 44 & 6 & 0 & -3.445156 & 0.374426 & 1.974959 \\
\hline 45 & 6 & 0 & -1.692339 & -1.205632 & 2.485995 \\
\hline 46 & 6 & 0 & -4.156173 & -0.052772 & 3.088692 \\
\hline 47 & 1 & 0 & -3.846850 & 1.171277 & 1.347771 \\
\hline 48 & 6 & 0 & -2.403155 & -1.624960 & 3.607390 \\
\hline 49 & 1 & 0 & -0.735107 & -1.664007 & 2.240406 \\
\hline 50 & 6 & 0 & -3.634403 & -1.054024 & 3.905833 \\
\hline 51 & 1 & 0 & -5.117587 & 0.400769 & 3.323783 \\
\hline 52 & 1 & 0 & -1.991159 & -2.404790 & 4.244977 \\
\hline 53 & 1 & 0 & -4.191382 & -1.384966 & 4.780980 \\
\hline 54 & 6 & 0 & 6.083904 & -0.311795 & 2.173095 \\
\hline 55 & 1 & 0 & 7.030282 & -0.859151 & 2.172855 \\
\hline 56 & 1 & 0 & 5.574139 & -0.456094 & 3.135497 \\
\hline 57 & 1 & 0 & 2.490585 & 4.821149 & -1.851141 \\
\hline 58 & 1 & 0 & 2.804720 & -1.390669 & -1.960356 \\
\hline 59 & 1 & 0 & 6.280555 & 0.764781 & 2.075130 \\
\hline
\end{tabular}

Zero-point correction=

Thermal correction to Energy=

0.460219 (Hartree/Particle)

alpy=

0.460219
0.492881

Thermal correction to Gibbs Free Energy=

Sum of electronic and zero-point Energies

Sum of electronic and thermal Energies=

Sum of electronic and thermal Enthalpies=

392242

-1673.984694
-1673.952032

$-1673.951088$

$-1674.052671$

M06 /6-311++G(d,p)-SDD/SMD//M06/6-31G(d)-LANL2DZ energy in toluene solvent = -1676.0229436

TS2-cis

\begin{tabular}{cccccc} 
Center & Atomic & Atomic & \multicolumn{2}{c}{ Coordinates (Angstroms) } & \\
Number & Number & Type & X & Y & Z \\
- \hdashline-1 & 6 & 0 & 5.474430 & -1.613084 & 0.784165 \\
2 & 6 & 0 & 4.667101 & -1.244232 & 1.851702 \\
3 & 6 & 0 & 3.397362 & -0.713528 & 1.618340
\end{tabular}




\begin{tabular}{|c|c|c|c|c|c|}
\hline 4 & 6 & 0 & 2.933563 & -0.568876 & 0.312972 \\
\hline 5 & 6 & 0 & 3.743128 & -0.947360 & -0.760841 \\
\hline 6 & 6 & 0 & 5.008784 & -1.464479 & -0.523360 \\
\hline 7 & 1 & 0 & 5.021820 & -1.363396 & 2.874640 \\
\hline 8 & 1 & 0 & 2.774468 & -0.430738 & 2.467314 \\
\hline 9 & 1 & 0 & 3.371606 & -0.857689 & -1.781891 \\
\hline 10 & 1 & 0 & 5.632654 & -1.768094 & -1.363176 \\
\hline 11 & 53 & 0 & 0.579913 & -2.769514 & -0.844439 \\
\hline 12 & 46 & 0 & 0.869717 & -0.242323 & 0.065594 \\
\hline 13 & 15 & 0 & -1.505550 & 0.216454 & 0.088986 \\
\hline 14 & 6 & 0 & 2.801609 & 2.144200 & -0.799832 \\
\hline 15 & 1 & 0 & 3.241584 & 1.646678 & -1.663058 \\
\hline 16 & 6 & 0 & 2.116238 & 1.468669 & 0.124537 \\
\hline 17 & 6 & 0 & 1.234781 & 1.615197 & 1.173562 \\
\hline 18 & 1 & 0 & 0.422727 & 2.335979 & 1.074139 \\
\hline 19 & 6 & 0 & 2.987165 & 3.604915 & -0.636858 \\
\hline 20 & 8 & 0 & 2.439499 & 4.248565 & 0.240264 \\
\hline 21 & 6 & 0 & -1.973618 & 0.153339 & 1.854224 \\
\hline 22 & 6 & 0 & -2.317036 & 1.286312 & 2.594510 \\
\hline 23 & 6 & 0 & -1.860560 & -1.084220 & 2.504042 \\
\hline 24 & 6 & 0 & -2.558575 & 1.182976 & 3.961935 \\
\hline 25 & 1 & 0 & -2.397492 & 2.256473 & 2.104863 \\
\hline 26 & 6 & 0 & -2.112666 & -1.183053 & 3.866194 \\
\hline 27 & 1 & 0 & -1.571442 & -1.971166 & 1.936458 \\
\hline 28 & 6 & 0 & -2.460370 & -0.049294 & 4.597934 \\
\hline 29 & 1 & 0 & -2.825636 & 2.072255 & 4.530503 \\
\hline 30 & 1 & 0 & -2.027935 & -2.149470 & 4.360100 \\
\hline 31 & 1 & 0 & -2.650197 & -0.128409 & 5.667026 \\
\hline 32 & 6 & 0 & -2.732930 & -0.821982 & -0.776648 \\
\hline 33 & 6 & 0 & -3.914747 & -1.260358 & -0.177018 \\
\hline 34 & 6 & 0 & -2.488870 & -1.116043 & -2.121506 \\
\hline 35 & 6 & 0 & -4.847040 & -1.979002 & -0.920110 \\
\hline 36 & 1 & 0 & -4.109990 & -1.042807 & 0.873133 \\
\hline 37 & 6 & 0 & -3.424989 & -1.826220 & -2.860966 \\
\hline 38 & 1 & 0 & -1.554376 & -0.796813 & -2.584316 \\
\hline 39 & 6 & 0 & -4.604295 & -2.259384 & -2.260210 \\
\hline 40 & 1 & 0 & -5.766333 & -2.320871 & -0.447371 \\
\hline 41 & 1 & 0 & -3.227105 & -2.054168 & -3.906667 \\
\hline 42 & 1 & 0 & -5.334058 & -2.823366 & -2.838824 \\
\hline 43 & 6 & 0 & -1.960738 & 1.892594 & -0.498585 \\
\hline 44 & 6 & 0 & -3.273647 & 2.367698 & -0.386441 \\
\hline 45 & 6 & 0 & -0.995356 & 2.697632 & -1.106962 \\
\hline 46 & 6 & 0 & -3.600978 & 3.637060 & -0.844769 \\
\hline 47 & 1 & 0 & -4.042420 & 1.737148 & 0.062192 \\
\hline 48 & 6 & 0 & -1.324547 & 3.970539 & -1.564632 \\
\hline 49 & 1 & 0 & 0.022836 & 2.325910 & -1.229146 \\
\hline 50 & 6 & 0 & -2.624470 & 4.442335 & -1.428940 \\
\hline 51 & 1 & 0 & -4.622612 & 4.000790 & -0.748724 \\
\hline 52 & 1 & 0 & -0.557394 & 4.593032 & -2.021672 \\
\hline 53 & 1 & 0 & -2.882685 & 5.439073 & -1.782519 \\
\hline 54 & 6 & 0 & 3.890005 & 4.249484 & -1.659127 \\
\hline 55 & 1 & 0 & 3.940311 & 5.327847 & -1.485597 \\
\hline 56 & 1 & 0 & 3.523760 & 4.055010 & -2.676508 \\
\hline 57 & 1 & 0 & 6.467330 & -2.021682 & 0.965049 \\
\hline 58 & 1 & 0 & 1.504327 & 1.324725 & 2.189398 \\
\hline 59 & 1 & 0 & 4.898736 & 3.818500 & -1.598174 \\
\hline
\end{tabular}

Zero-point correction= 0.460241 (Hartree/Particle)

Thermal correction to Energy=

0.492955

Thermal correction to Enthalpy=

Thermal correction to Gibbs Free Energy=

Sum of electronic and zero-point Energies=

Sum of electronic and thermal Energies

0.493899

0.391151

$-1673.981543$

$-1673.948829$

$-1673.947885$

Sum of electronic and thermal Free Energies

$-1674.050634$

M06 /6-311++G(d,p)-SDD/SMD//M06/6-31G(d)-LANL2DZ energy in toluene solvent $=-1676.0205692$

TS2-a

\begin{tabular}{rrrrrr} 
Center & Atomic & Atomic & \multicolumn{2}{c}{ Coordinates (Angstroms) } \\
Number & Number & Type & X & Y & $Z$ \\
-1 & 53 & 0 & 0.513680 & -1.150453 & -2.651867 \\
2 & 15 & 0 & -2.106469 & 0.494989 & 0.164801 \\
3 & 15 & 0 & 1.771136 & 1.042683 & 0.109832 \\
4 & 6 & 0 & 1.513599 & 2.584223 & -0.859352 \\
5 & 6 & 0 & 0.905752 & 2.529145 & -2.117336 \\
6 & 6 & 0 & 1.984013 & 3.814685 & -0.383919 \\
7 & 6 & 0 & 0.803982 & 3.675530 & -2.897831 \\
8 & 1 & 0 & 0.520189 & 1.581382 & -2.492753 \\
9 & 6 & 0 & 1.861037 & 4.961888 & -1.160519 \\
10 & 1 & 0 & 2.456173 & 3.881417 & 0.595388 \\
11 & 6 & 0 & 1.279931 & 4.892564 & -2.423271 \\
12 & 1 & 0 & 0.331264 & 3.612501 & -3.876673 \\
13 & 1 & 0 & 2.233444 & 5.911479 & -0.778789 \\
14 & 1 & 0 & 1.194328 & 5.790363 & -3.034194 \\
15 & 6 & 0 & 2.121202 & 1.720871 & 1.782186 \\
16 & 6 & 0 & 1.023673 & 2.130360 & 2.546735 \\
17 & 6 & 0 & 3.406571 & 1.895899 & 2.300164 \\
18 & 6 & 0 & 1.201162 & 2.693724 & 3.803323 \\
19 & 1 & 0 & 0.014432 & 2.002852 & 2.153236 \\
20 & 6 & 0 & 3.584671 & 2.454047 & 3.564050 \\
21 & 1 & 0 & 4.275502 & 1.600894 & 1.713805
\end{tabular}




\begin{tabular}{|c|c|c|c|c|c|}
\hline 22 & 6 & 0 & 2.486072 & 2.851640 & 4.317822 \\
\hline 23 & 1 & 0 & 0.332478 & 3.005139 & 4.382183 \\
\hline 24 & 1 & 0 & 4.591548 & 2.578890 & 3.959154 \\
\hline 25 & 1 & 0 & 2.630013 & 3.285925 & 5.305846 \\
\hline 26 & 6 & 0 & 3.392887 & 0.416054 & -0.461101 \\
\hline 27 & 6 & 0 & 4.043031 & -0.570687 & 0.291782 \\
\hline 28 & 6 & 0 & 3.957513 & 0.828752 & -1.669770 \\
\hline 29 & 6 & 0 & 5.244685 & -1.114166 & -0.146363 \\
\hline 30 & 1 & 0 & 3.627520 & -0.915943 & 1.238264 \\
\hline 31 & 6 & 0 & 5.153522 & 0.272131 & -2.111693 \\
\hline 32 & 1 & 0 & 3.458223 & 1.584855 & -2.273829 \\
\hline 33 & 6 & 0 & 5.800253 & -0.697100 & -1.352578 \\
\hline 34 & 1 & 0 & 5.746416 & -1.863248 & 0.465609 \\
\hline 35 & 1 & 0 & 5.580499 & 0.601588 & -3.057356 \\
\hline 36 & 1 & 0 & 6.738636 & -1.126224 & -1.700338 \\
\hline 37 & 6 & 0 & -2.336720 & 1.960111 & -0.924343 \\
\hline 38 & 6 & 0 & -2.892405 & 1.851774 & -2.200760 \\
\hline 39 & 6 & 0 & -1.870267 & 3.209631 & -0.495729 \\
\hline 40 & 6 & 0 & -3.002211 & 2.973487 & -3.018551 \\
\hline 41 & 1 & 0 & -3.257711 & 0.889610 & -2.557427 \\
\hline 42 & 6 & 0 & -1.994223 & 4.330037 & -1.306705 \\
\hline 43 & 1 & 0 & -1.419598 & 3.316783 & 0.492519 \\
\hline 44 & 6 & 0 & -2.564500 & 4.215211 & -2.571361 \\
\hline 45 & 1 & 0 & -3.445587 & 2.872954 & -4.008132 \\
\hline 46 & 1 & 0 & -1.623633 & 5.291841 & -0.954193 \\
\hline 47 & 1 & 0 & -2.658277 & 5.091966 & -3.210709 \\
\hline 48 & 6 & 0 & -2.675187 & 1.166269 & 1.786664 \\
\hline 49 & 6 & 0 & -2.066551 & 0.705724 & 2.956825 \\
\hline 50 & 6 & 0 & -3.717070 & 2.096617 & 1.897181 \\
\hline 51 & 6 & 0 & -2.486547 & 1.151570 & 4.207810 \\
\hline 52 & 1 & 0 & -1.234241 & 0.004876 & 2.880184 \\
\hline 53 & 6 & 0 & -4.136323 & 2.545916 & 3.143682 \\
\hline 54 & 1 & 0 & -4.203083 & 2.471979 & 0.996391 \\
\hline 55 & 6 & 0 & -3.522787 & 2.073292 & 4.301999 \\
\hline 56 & 1 & 0 & -1.995327 & 0.782627 & 5.107085 \\
\hline 57 & 1 & 0 & -4.947011 & 3.269625 & 3.212761 \\
\hline 58 & 1 & 0 & -3.852971 & 2.428092 & 5.277048 \\
\hline 59 & 6 & 0 & -3.493220 & -0.618797 & -0.312302 \\
\hline 60 & 6 & 0 & -4.689782 & -0.712349 & 0.404860 \\
\hline 61 & 6 & 0 & -3.302149 & -1.451337 & -1.422321 \\
\hline 62 & 6 & 0 & -5.679006 & -1.608166 & 0.010027 \\
\hline 63 & 1 & 0 & -4.855459 & -0.092865 & 1.284675 \\
\hline 64 & 6 & 0 & -4.294805 & -2.341229 & -1.815784 \\
\hline 65 & 1 & 0 & -2.358294 & -1.418538 & -1.970939 \\
\hline 66 & 6 & 0 & -5.486012 & -2.421663 & -1.101000 \\
\hline 67 & 1 & 0 & -6.605968 & -1.669449 & 0.578738 \\
\hline 68 & 1 & 0 & -4.124802 & -2.984656 & -2.677730 \\
\hline 69 & 1 & 0 & -6.260815 & -3.123770 & -1.405757 \\
\hline 70 & 6 & 0 & -2.734712 & -4.763071 & 0.408869 \\
\hline 71 & 6 & 0 & -2.699820 & -3.830344 & 1.436685 \\
\hline 72 & 6 & 0 & -1.719501 & -2.838935 & 1.450158 \\
\hline 73 & 6 & 0 & -0.775618 & -2.759956 & 0.423325 \\
\hline 74 & 6 & 0 & -0.798172 & -3.719947 & -0.592391 \\
\hline 75 & 6 & 0 & -1.772376 & -4.709412 & -0.598245 \\
\hline 76 & 1 & 0 & -3.450698 & -3.850315 & 2.225673 \\
\hline 77 & 1 & 0 & -1.732599 & -2.110413 & 2.260164 \\
\hline 78 & 1 & 0 & -0.061489 & -3.684794 & -1.392650 \\
\hline 79 & 1 & 0 & -1.781341 & -5.443215 & -1.403675 \\
\hline 80 & 46 & 0 & 0.182342 & -0.852162 & 0.188084 \\
\hline 81 & 6 & 0 & 2.116002 & -3.286385 & 0.644836 \\
\hline 82 & 1 & 0 & 2.051219 & -3.653478 & -0.379887 \\
\hline 83 & 6 & 0 & 1.223075 & -2.406215 & 1.110997 \\
\hline 84 & 6 & 0 & 1.002118 & -1.490774 & 2.132583 \\
\hline 85 & 1 & 0 & 1.812872 & -0.838993 & 2.461089 \\
\hline 86 & 6 & 0 & 3.225901 & -3.746625 & 1.498076 \\
\hline 87 & 8 & 0 & 3.446308 & -3.291851 & 2.609814 \\
\hline 88 & 6 & 0 & 4.115807 & -4.793303 & 0.874108 \\
\hline 89 & 1 & 0 & 4.942261 & -5.033927 & 1.548732 \\
\hline 90 & 1 & 0 & 4.510729 & -4.432265 & -0.086168 \\
\hline 91 & 1 & 0 & -3.502874 & -5.534662 & 0.393634 \\
\hline 92 & 1 & 0 & 0.211886 & -1.658752 & 2.863326 \\
\hline 93 & 1 & 0 & 3.539935 & -5.703084 & 0.655642 \\
\hline
\end{tabular}

Zero-point correction= 0.737604 (Hartree/Particle) Thermal correction to Energy $=$ Thermal correction to Enthalpy=

Thermal correction to Gibbs Free Energy=

Sum of electronic and zero-point Energies= Sum of electronic and thermal Energies=

um of electronic and thermal Enthalpies= 0.786584 0.787529
0.653825

$-2709.425109$

$-2709.376129$ $-2709.375185$

M06 /6-311++G(d,p)-SDD/SMD//M06/6-31G(d)-LANL2DZ energy in toluene solvent $=-2711.9613095$

TS3

\begin{tabular}{|c|c|c|c|c|c|}
\hline \multirow{2}{*}{$\begin{array}{l}\text { Center } \\
\text { Number }\end{array}$} & \multirow{2}{*}{$\begin{array}{l}\text { Atomic } \\
\text { Number }\end{array}$} & \multirow{2}{*}{$\begin{array}{l}\text { Atomic } \\
\text { Type }\end{array}$} & \multicolumn{3}{|c|}{ Coordinates (Angstroms) } \\
\hline & & & $\mathrm{X}$ & $\mathrm{Y}$ & \\
\hline 1 & 6 & 0 & -4.527287 & 2.953290 & 1.241035 \\
\hline 2 & 6 & 0 & -4.726162 & 1.655566 & 1.706125 \\
\hline 3 & 6 & 0 & -4.104009 & 0.583607 & 1.080856 \\
\hline 4 & 6 & 0 & -3.262467 & 0.786581 & -0.020847 \\
\hline 5 & 6 & 0 & -3.060452 & 2.098215 & -0.470294 \\
\hline
\end{tabular}




\begin{tabular}{|c|c|c|c|c|c|}
\hline 6 & 6 & 0 & -3.692961 & 3.168668 & 0.148763 \\
\hline 7 & 1 & 0 & -5.380664 & 1.474840 & 2.557417 \\
\hline 8 & 1 & 0 & -4.288743 & -0.428454 & 1.438630 \\
\hline 9 & 1 & 0 & -2.372813 & 2.282159 & -1.297417 \\
\hline 10 & 1 & 0 & -3.515254 & 4.178460 & -0.219185 \\
\hline 11 & 53 & 0 & 0.772406 & -3.155262 & 0.806774 \\
\hline 12 & 46 & 0 & -0.524723 & -0.971065 & -0.148753 \\
\hline 13 & 15 & 0 & 1.160612 & 0.674673 & -0.107571 \\
\hline 14 & 6 & 0 & -2.532979 & -1.687333 & -0.175235 \\
\hline 15 & 1 & 0 & -2.761402 & -1.942284 & 0.859893 \\
\hline 16 & 6 & 0 & -2.589045 & -0.339218 & -0.693450 \\
\hline 17 & 6 & 0 & -2.412600 & -0.340281 & -2.114077 \\
\hline 18 & 1 & 0 & -1.529330 & -0.748107 & -2.596601 \\
\hline 19 & 6 & 0 & -2.804692 & -2.645896 & -1.221284 \\
\hline 20 & 8 & 0 & -3.031474 & -2.220943 & -2.379603 \\
\hline 21 & 6 & 0 & 0.537402 & 2.307362 & -0.677300 \\
\hline 22 & 6 & 0 & 0.217163 & 3.333844 & 0.211698 \\
\hline 23 & 6 & 0 & 0.291314 & 2.493971 & -2.044434 \\
\hline 24 & 6 & 0 & -0.326443 & 4.528796 & -0.256560 \\
\hline 25 & 1 & 0 & 0.392245 & 3.202641 & 1.279361 \\
\hline 26 & 6 & 0 & -0.243498 & 3.687705 & -2.510729 \\
\hline 27 & 1 & 0 & 0.533020 & 1.697660 & -2.751590 \\
\hline 28 & 6 & 0 & -0.553605 & 4.711609 & -1.615080 \\
\hline 29 & 1 & 0 & -0.569808 & 5.320882 & 0.450145 \\
\hline 30 & 1 & 0 & -0.414995 & 3.824261 & -3.577589 \\
\hline 31 & 1 & 0 & -0.969812 & 5.649053 & -1.980866 \\
\hline 32 & 6 & 0 & 1.746059 & 1.024837 & 1.589774 \\
\hline 33 & 6 & 0 & 2.926809 & 1.726082 & 1.849944 \\
\hline 34 & 6 & 0 & 0.932782 & 0.631323 & 2.655377 \\
\hline 35 & 6 & 0 & 3.283087 & 2.034559 & 3.157432 \\
\hline 36 & 1 & 0 & 3.574627 & 2.026694 & 1.026523 \\
\hline 37 & 6 & 0 & 1.287588 & 0.947060 & 3.963084 \\
\hline 38 & 1 & 0 & 0.023615 & 0.063152 & 2.448502 \\
\hline 39 & 6 & 0 & 2.462030 & 1.648699 & 4.214041 \\
\hline 40 & 1 & 0 & 4.207175 & 2.576160 & 3.353405 \\
\hline 41 & 1 & 0 & 0.649475 & 0.634267 & 4.787575 \\
\hline 42 & 1 & 0 & 2.743302 & 1.890720 & 5.237808 \\
\hline 43 & 6 & 0 & 2.677601 & 0.459468 & -1.111875 \\
\hline 44 & 6 & 0 & 3.091863 & -0.838264 & -1.423891 \\
\hline 45 & 6 & 0 & 3.443051 & 1.547030 & -1.552787 \\
\hline 46 & 6 & 0 & 4.257391 & -1.045033 & -2.155643 \\
\hline 47 & 1 & 0 & 2.498337 & -1.688870 & -1.085623 \\
\hline 48 & 6 & 0 & 4.606789 & 1.335717 & -2.282951 \\
\hline 49 & 1 & 0 & 3.121867 & 2.566101 & -1.333843 \\
\hline 50 & 6 & 0 & 5.015231 & 0.038812 & -2.584811 \\
\hline 51 & 1 & 0 & 4.569260 & -2.060757 & -2.392252 \\
\hline 52 & 1 & 0 & 5.195273 & 2.187554 & -2.620442 \\
\hline 53 & 1 & 0 & 5.925697 & -0.125076 & -3.159276 \\
\hline 54 & 6 & 0 & -2.808916 & -4.108125 & -0.936190 \\
\hline 55 & 1 & 0 & -3.522287 & -4.332466 & -0.132773 \\
\hline 56 & 1 & 0 & -1.813599 & -4.401001 & -0.567357 \\
\hline 57 & 1 & 0 & -2.956600 & 0.395517 & -2.716630 \\
\hline 58 & 1 & 0 & -3.065287 & -4.681837 & -1.830372 \\
\hline 59 & 1 & 0 & -5.017244 & 3.792183 & 1.732451 \\
\hline
\end{tabular}

Zero-point correction=

Thermal correction to Energy=

0.463739 (Hartree/Particle)

Thermal correction to Enthalpy=

0.495359

Thermal correction to Gibbs Free Energy=

Sum of electronic and zero-point Energies=

Sum of electronic and thermal Energies=

Sum of electronic and thermal Enthalpies=

Sum of electronic and thermal Free Energies=

0.396347

$-1674.022771$

$-1674.022771$

-1673.991151
-1673.990207

$-1674.090163$

M06 /6-311++G(d,p)-SDD/SMD//M06/6-31G(d)-LANL2DZ energy in toluene solvent $=-1676.0567997$

\section{TS4}

\begin{tabular}{rrrrrr} 
Center & $\begin{array}{r}\text { Atomic } \\
\text { Number }\end{array}$ & Number & $\begin{array}{r}\text { Atomic } \\
\text { Type }\end{array}$ & \multicolumn{2}{c}{ Coordinates (Angstroms) } \\
-1 & 6 & 0 & 3.768719 & 1.012400 & -2.866770 \\
2 & 6 & 0 & 3.147728 & -0.209030 & -3.126116 \\
3 & 6 & 0 & 2.679151 & -0.991486 & -2.080604 \\
4 & 6 & 0 & 2.823329 & -0.572361 & -0.747679 \\
5 & 6 & 0 & 3.451924 & 0.653053 & -0.499652 \\
6 & 6 & 0 & 3.917404 & 1.436055 & -1.550912 \\
7 & 1 & 0 & 3.019829 & -0.552652 & -4.151324 \\
8 & 1 & 0 & 2.168145 & -1.928612 & -2.302680 \\
9 & 1 & 0 & 3.577300 & 1.003787 & 0.524266 \\
10 & 1 & 0 & 4.396775 & 2.389564 & -1.332644 \\
11 & 53 & 0 & -2.299277 & -2.216747 & -1.070098 \\
12 & 46 & 0 & 0.070149 & -1.457666 & -0.039481 \\
13 & 15 & 0 & -0.534729 & 0.884420 & 0.280530 \\
14 & 6 & 0 & 2.522854 & -1.186822 & 1.780591 \\
15 & 1 & 0 & 2.735579 & -0.240149 & 2.264988 \\
16 & 6 & 0 & 2.332256 & -1.404525 & 0.362288 \\
17 & 6 & 0 & 2.014628 & -2.786624 & 0.265024 \\
18 & 1 & 0 & 0.278989 & -3.033619 & -0.249475 \\
19 & 6 & 0 & 2.334581 & -2.372097 & 2.405047 \\
20 & 8 & 0 & 2.072708 & -3.362806 & 1.499608 \\
21 & 6 & 0 & -1.002006 & 1.621083 & -1.324679 \\
22 & 6 & 0 & -2.230354 & 2.237093 & -1.564835 \\
23 & 6 & 0 & -0.086572 & 1.486893 & -2.374711
\end{tabular}




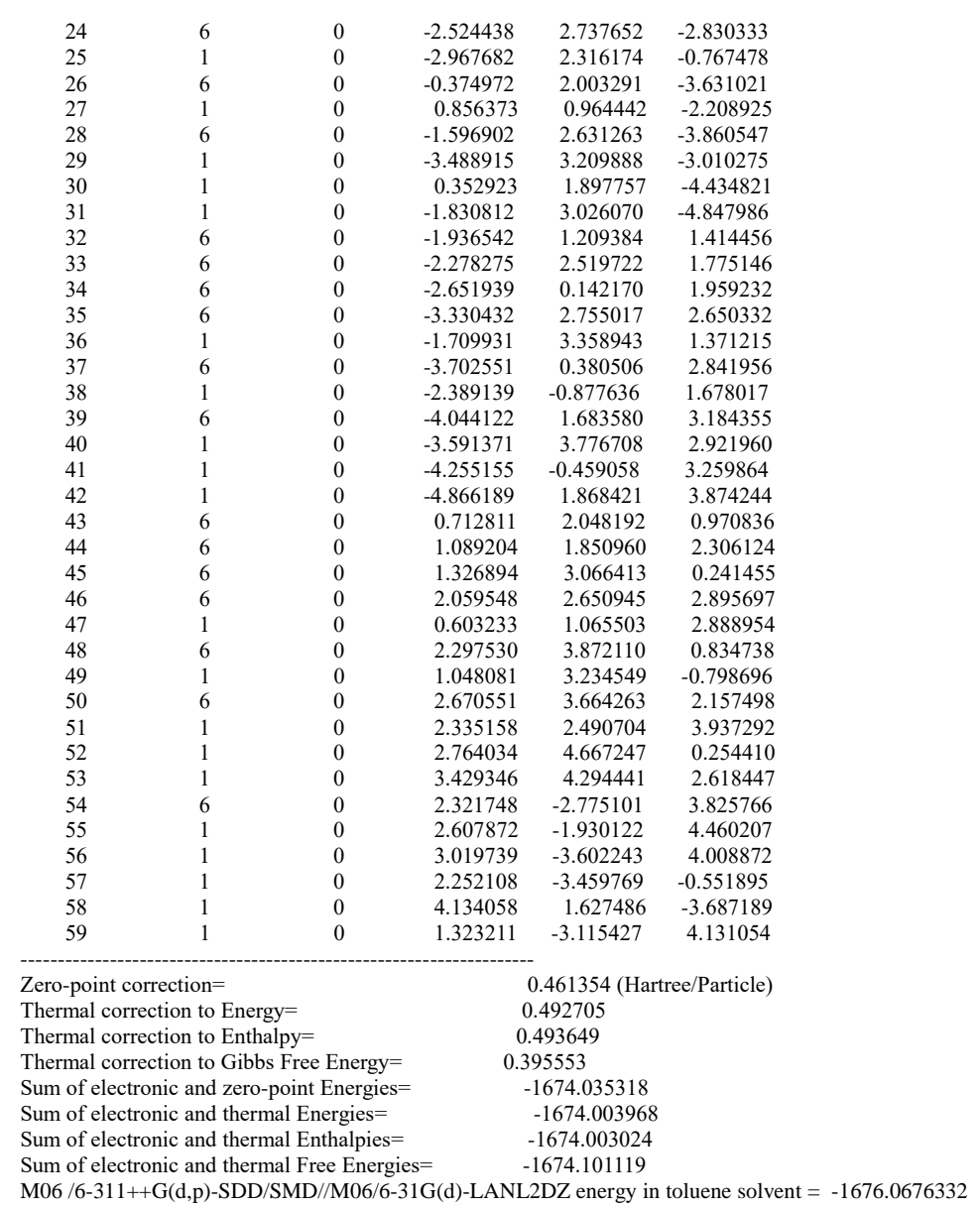

TS5

\begin{tabular}{|c|c|c|c|c|c|}
\hline \multirow{2}{*}{$\begin{array}{l}\text { Center } \\
\text { Number }\end{array}$} & \multirow{2}{*}{$\begin{array}{l}\text { Atomic } \\
\text { Number }\end{array}$} & \multirow{2}{*}{$\begin{array}{l}\text { Atomic } \\
\text { Type }\end{array}$} & \multicolumn{3}{|c|}{ Coordinates (Angstroms) } \\
\hline & & & $\mathrm{X}$ & $\mathrm{Y}$ & Z \\
\hline 1 & 53 & 0 & 0.512807 & -3.873299 & 0.012775 \\
\hline 2 & 15 & 0 & 2.120146 & 0.413568 & -0.003785 \\
\hline 3 & 46 & 0 & 0.028382 & -0.568859 & -0.058191 \\
\hline 4 & 15 & 0 & -2.261603 & 0.156304 & -0.036151 \\
\hline 5 & 1 & 0 & 0.856985 & -2.077760 & 0.027777 \\
\hline 6 & 6 & 0 & 1.987440 & 2.156661 & -0.559427 \\
\hline 7 & 6 & 0 & 1.125470 & 3.004712 & 0.149090 \\
\hline 8 & 6 & 0 & 2.625408 & 2.640116 & -1.702676 \\
\hline 9 & 6 & 0 & 0.925600 & 4.313617 & -0.264689 \\
\hline 10 & 1 & 0 & 0.599150 & 2.626685 & 1.028359 \\
\hline 11 & 6 & 0 & 2.411279 & 3.951104 & -2.125332 \\
\hline 12 & 1 & 0 & 3.293680 & 1.992899 & -2.270529 \\
\hline 13 & 6 & 0 & 1.567718 & 4.789804 & -1.406963 \\
\hline 14 & 1 & 0 & 0.247423 & 4.958195 & 0.292383 \\
\hline 15 & 1 & 0 & 2.913592 & 4.316202 & -3.020085 \\
\hline 16 & 1 & 0 & 1.401620 & 5.813614 & -1.739297 \\
\hline 17 & 6 & 0 & 3.465293 & -0.273367 & -1.035640 \\
\hline 18 & 6 & 0 & 4.817018 & 0.001228 & -0.806563 \\
\hline 19 & 6 & 0 & 3.103167 & -1.073104 & -2.122490 \\
\hline 20 & 6 & 0 & 5.788404 & -0.512253 & -1.657628 \\
\hline 21 & 1 & 0 & 5.110155 & 0.615557 & 0.045409 \\
\hline 22 & 6 & 0 & 4.076020 & -1.580682 & -2.977824 \\
\hline 23 & 1 & 0 & 2.048164 & -1.300154 & -2.288674 \\
\hline 24 & 6 & 0 & 5.418430 & -1.300458 & -2.744862 \\
\hline 25 & 1 & 0 & 6.839769 & -0.298739 & -1.470702 \\
\hline 26 & 1 & 0 & 3.784282 & -2.204289 & -3.820961 \\
\hline 27 & 1 & 0 & 6.181479 & -1.703120 & -3.409223 \\
\hline 28 & 6 & 0 & 2.852861 & 0.551058 & 1.669499 \\
\hline 29 & 6 & 0 & 2.618479 & -0.495792 & 2.566826 \\
\hline 30 & 6 & 0 & 3.636667 & 1.636236 & 2.075261 \\
\hline 31 & 6 & 0 & 3.171064 & -0.465490 & 3.842843 \\
\hline 32 & 1 & 0 & 1.993845 & -1.336943 & 2.259653 \\
\hline 33 & 6 & 0 & 4.183144 & 1.667287 & 3.354115 \\
\hline 34 & 1 & 0 & 3.812475 & 2.465895 & 1.389642 \\
\hline 35 & 6 & 0 & 3.952251 & 0.616560 & 4.237449 \\
\hline 36 & 1 & 0 & 2.983300 & -1.286040 & 4.533124 \\
\hline 37 & 1 & 0 & 4.789366 & 2.517792 & 3.662456 \\
\hline 38 & 1 & 0 & 4.378361 & 0.644721 & 5.239129 \\
\hline 39 & 6 & 0 & -2.340442 & 1.846347 & -0.751397 \\
\hline 40 & 6 & 0 & -1.629821 & 2.068285 & -1.937543 \\
\hline 41 & 6 & 0 & -3.027484 & 2.909673 & -0.162384 \\
\hline
\end{tabular}




$\begin{array}{rrrrrr}42 & 6 & 0 & -1.623253 & 3.321817 & -2.533898 \\ 43 & 1 & 0 & -1.058770 & 1.249093 & -2.379707 \\ 44 & 6 & 0 & -3.007623 & 4.171298 & -0.754372 \\ 45 & 1 & 0 & -3.577918 & 2.755521 & 0.765822 \\ 46 & 6 & 0 & -2.310505 & 4.378138 & -1.939637 \\ 47 & 1 & 0 & -1.057960 & 3.482409 & -3.450760 \\ 48 & 1 & 0 & -3.542911 & 4.995142 & -0.283963 \\ 49 & 1 & 0 & -2.294017 & 5.366119 & -2.397882 \\ 50 & 6 & 0 & -2.970040 & 0.365568 & 1.640658 \\ 51 & 6 & 0 & -4.342047 & 0.332679 & 1.904189 \\ 52 & 6 & 0 & -2.077296 & 0.596097 & 2.692635 \\ 53 & 6 & 0 & -4.812242 & 0.534687 & 3.197881 \\ 54 & 1 & 0 & -5.046731 & 0.144582 & 1.093395 \\ 55 & 6 & 0 & -2.549267 & 0.804981 & 3.983769 \\ 56 & 1 & 0 & -1.003098 & 0.595563 & 2.488298 \\ 57 & 6 & 0 & -3.917498 & 0.773162 & 4.237259 \\ 58 & 1 & 0 & -5.882587 & 0.502571 & 3.395757 \\ 59 & 1 & 0 & -1.845229 & 0.980654 & 4.795501 \\ 60 & 1 & 0 & -4.288447 & 0.926369 & 5.249523 \\ 61 & 6 & 0 & -3.551175 & -0.763754 & -0.954135 \\ 62 & 6 & 0 & -3.454813 & -2.158892 & -0.964813 \\ 63 & 6 & 0 & -4.612424 & -0.143275 & -1.620127 \\ 64 & 6 & 0 & -4.416947 & -2.922813 & -1.615692 \\ 65 & 1 & 0 & -2.612957 & -2.646544 & -0.468218 \\ 66 & 6 & 0 & -5.568470 & -0.910489 & -2.278151 \\ 67 & 1 & 0 & -4.688012 & 0.944884 & -1.626342 \\ 68 & 6 & 0 & -5.473188 & -2.299221 & -2.273832 \\ 69 & 1 & 0 & -4.332591 & -4.008100 & -1.619190 \\ 70 & 1 & 0 & -6.390607 & -0.420749 & -2.798119 \\ 71 & 1 & 0 & -6.221417 & -2.896941 & -2.792187\end{array}$

0.555186 (Hartree/Particle)

Thermal correction to Energy=

Thermal correction to Enthalpy=

0.593681

Thermal correction to Gibbs Free Energy=

Sero-point Energies=

Sum of electronic and thermal Energies=

0.477967

Sum of electronic and thermal Enthalpies $=$

$-2209.594308$

Sum of electronic and thermal Free Energies=

$-2209.593364$

$-2209.709078$

M06 /6-311++G(d,p)-SDD/SMD//M06/6-31G(d)-LANL2DZ energy in toluene solvent $=-2211.8387453$

TS6

\begin{tabular}{|c|c|c|c|c|c|}
\hline \multirow{2}{*}{$\begin{array}{l}\text { Center } \\
\text { Number }\end{array}$} & \multirow{2}{*}{$\begin{array}{l}\text { Atomic } \\
\text { Number }\end{array}$} & \multirow{2}{*}{$\begin{array}{l}\text { Atomic } \\
\text { Type }\end{array}$} & \multicolumn{3}{|c|}{ Coordinates (Angstroms) } \\
\hline & & & $\mathrm{X}$ & $\mathrm{Y}$ & \\
\hline 1 & 6 & 0 & 0.957984 & 4.849136 & -0.072115 \\
\hline 2 & 6 & 0 & 0.606410 & 4.138571 & -1.216173 \\
\hline 3 & 6 & 0 & 0.618575 & 2.744694 & -1.208401 \\
\hline 4 & 6 & 0 & 0.970189 & 2.045221 & -0.052433 \\
\hline 5 & 6 & 0 & 1.339391 & 2.762628 & 1.086716 \\
\hline 6 & 6 & 0 & 1.326207 & 4.157692 & 1.078471 \\
\hline 7 & 1 & 0 & 0.312108 & 4.670092 & -2.121265 \\
\hline 8 & 1 & 0 & 0.318905 & 2.205411 & -2.110419 \\
\hline 9 & 1 & 0 & 1.637049 & 2.234520 & 1.994755 \\
\hline 10 & 1 & 0 & 1.609356 & 4.705377 & 1.977678 \\
\hline 11 & 53 & 0 & 1.166378 & -2.763455 & -0.011368 \\
\hline 12 & 46 & 0 & 0.971609 & -0.002706 & -0.015146 \\
\hline 13 & 15 & 0 & -1.411681 & -0.028932 & 0.055460 \\
\hline 14 & 6 & 0 & 4.063112 & 0.201114 & 0.870454 \\
\hline 15 & 1 & 0 & 4.010804 & 0.248449 & 1.955054 \\
\hline 16 & 6 & 0 & 3.034709 & 0.111434 & 0.013592 \\
\hline 17 & 6 & 0 & 3.235983 & 0.056567 & -1.352596 \\
\hline 18 & 1 & 0 & 3.213221 & -0.902616 & -1.872485 \\
\hline 19 & 6 & 0 & 5.335673 & 0.201680 & 0.148137 \\
\hline 20 & 8 & 0 & 5.343771 & 0.144612 & -1.088346 \\
\hline 21 & 6 & 0 & -2.112389 & -1.166563 & -1.189253 \\
\hline 22 & 6 & 0 & -3.145942 & -2.057965 & -0.904723 \\
\hline 23 & 6 & 0 & -1.567007 & -1.135310 & -2.477417 \\
\hline 24 & 6 & 0 & -3.633590 & -2.901179 & -1.899432 \\
\hline 25 & 1 & 0 & -3.562849 & -2.110598 & 0.100175 \\
\hline 26 & 6 & 0 & -2.064954 & -1.966827 & -3.471410 \\
\hline 27 & 1 & 0 & -0.734146 & -0.462618 & -2.693361 \\
\hline 28 & 6 & 0 & -3.098674 & -2.854115 & -3.181455 \\
\hline 29 & 1 & 0 & -4.432858 & -3.602692 & -1.666512 \\
\hline 30 & 1 & 0 & -1.633775 & -1.935422 & -4.470445 \\
\hline 31 & 1 & 0 & -3.479653 & -3.518038 & -3.955648 \\
\hline 32 & 6 & 0 & -2.126360 & -0.526946 & 1.661539 \\
\hline 33 & 6 & 0 & -1.348187 & -1.232756 & 2.582066 \\
\hline 34 & 6 & 0 & -3.457712 & -0.224517 & 1.975227 \\
\hline 35 & 6 & 0 & -1.895981 & -1.634026 & 3.797183 \\
\hline 36 & 1 & 0 & -0.315528 & -1.482266 & 2.335781 \\
\hline 37 & 6 & 0 & -4.001275 & -0.630425 & 3.187678 \\
\hline 38 & 1 & 0 & -4.069716 & 0.335255 & 1.267279 \\
\hline 39 & 6 & 0 & -3.219468 & -1.334861 & 4.100261 \\
\hline 40 & 1 & 0 & -1.282279 & -2.185948 & 4.506735 \\
\hline 41 & 1 & 0 & -5.037491 & -0.392651 & 3.422765 \\
\hline 42 & 1 & 0 & -3.645207 & -1.650407 & 5.051556 \\
\hline 43 & 6 & 0 & -2.242385 & 1.568746 & -0.281798 \\
\hline 44 & 6 & 0 & -2.915372 & 1.827807 & -1.475888 \\
\hline 45 & 6 & 0 & -2.105131 & 2.593619 & 0.661527 \\
\hline 46 & 6 & 0 & -3.436532 & 3.095564 & -1.726187 \\
\hline 47 & 1 & 0 & -3.036447 & 1.039496 & -2.217699 \\
\hline
\end{tabular}




$\begin{array}{rrrrrr}48 & 6 & 0 & -2.626750 & 3.854233 & 0.411060 \\ 49 & 1 & 0 & -1.565078 & 2.406824 & 1.590485 \\ 50 & 6 & 0 & -3.290646 & 4.109338 & -0.787559 \\ 51 & 1 & 0 & -3.961084 & 3.286391 & -2.661229 \\ 52 & 1 & 0 & -2.501832 & 4.644799 & 1.149183 \\ 53 & 1 & 0 & -3.695080 & 5.100632 & -0.986385 \\ 54 & 6 & 0 & 6.613332 & 0.264171 & 0.923811 \\ 55 & 1 & 0 & 7.476792 & 0.287432 & 0.254578 \\ 56 & 1 & 0 & 6.683167 & -0.609357 & 1.585378 \\ 57 & 1 & 0 & 6.617183 & 1.152613 & 1.568488 \\ 58 & 1 & 0 & 3.161706 & 0.961508 & -1.957930 \\ 59 & 1 & 0 & 0.946487 & 5.938162 & -0.077715\end{array}$

Zero-point correction $=\quad 0.460386($ Hartree/Particle $)$

Thermal correction to Energy $=\quad 0.493070$

Thermal correction to Enthalpy= $\quad 0.494015$

Thermal correction to Gibbs Free Energy= 0.391330

Sum of electronic and zero-point Energies $=\quad-1673.985367$

Sum of electronic and thermal Energies $=\quad-1673.952683$

Sum of electronic and thermal Enthalpies $=\quad-1673.951738$

Sum of electronic and thermal Free Energies $=\quad-1674.054423$

M06 /6-311++G(d,p)-SDD/SMD//M06/6-31G(d)-LANL2DZ energy in toluene solvent $=-1676.0225462$

TS6

\begin{tabular}{|c|c|c|c|c|c|}
\hline \multirow{2}{*}{$\begin{array}{l}\text { Center } \\
\text { Number }\end{array}$} & \multirow{2}{*}{$\begin{array}{l}\text { Atomic } \\
\text { Number }\end{array}$} & \multirow{2}{*}{$\begin{array}{l}\text { Atomic } \\
\text { Type }\end{array}$} & \multicolumn{3}{|c|}{ Coordinates (Angstroms) } \\
\hline & & & $X$ & $\mathrm{Y}$ & Z \\
\hline 1 & 6 & 0 & -2.283653 & 4.885303 & 0.205744 \\
\hline 2 & 6 & 0 & -2.420130 & 4.164986 & -0.977685 \\
\hline 3 & 6 & 0 & -1.836171 & 2.905536 & -1.103716 \\
\hline 4 & 6 & 0 & -1.113337 & 2.353200 & -0.044855 \\
\hline 5 & 6 & 0 & -0.962310 & 3.085792 & 1.132690 \\
\hline 6 & 6 & 0 & -1.552661 & 4.343913 & 1.259363 \\
\hline 7 & 1 & 0 & -2.993781 & 4.578684 & -1.807212 \\
\hline 8 & 1 & 0 & -1.974926 & 2.341312 & -2.029244 \\
\hline 9 & 1 & 0 & -0.383750 & 2.675972 & 1.962689 \\
\hline 10 & 1 & 0 & -1.434404 & 4.903679 & 2.187354 \\
\hline 11 & 53 & 0 & 1.095698 & -1.903309 & -0.526362 \\
\hline 12 & 46 & 0 & -0.249440 & 0.508205 & -0.226184 \\
\hline 13 & 15 & 0 & -2.387292 & -0.508331 & 0.100812 \\
\hline 14 & 6 & 0 & 2.564294 & 1.809405 & 0.581231 \\
\hline 15 & 1 & 0 & 2.605425 & 1.606045 & 1.648250 \\
\hline 16 & 6 & 0 & 1.576986 & 1.481884 & -0.262328 \\
\hline 17 & 6 & 0 & 1.630929 & 1.763176 & -1.610899 \\
\hline 18 & 1 & 0 & 1.972201 & 1.003908 & -2.317785 \\
\hline 19 & 6 & 0 & 3.679782 & 2.435930 & -0.141300 \\
\hline 20 & 8 & 0 & 3.572817 & 2.666405 & -1.352192 \\
\hline 21 & 6 & 0 & -2.768923 & -1.838902 & -1.090836 \\
\hline 22 & 6 & 0 & -3.537616 & -2.952106 & -0.749624 \\
\hline 23 & 6 & 0 & -2.309708 & -1.694488 & -2.403022 \\
\hline 24 & 6 & 0 & -3.842367 & -3.909696 & -1.711925 \\
\hline 25 & 1 & 0 & -3.889950 & -3.081225 & 0.273371 \\
\hline 26 & 6 & 0 & -2.625659 & -2.645693 & -3.365127 \\
\hline 27 & 1 & 0 & -1.685755 & -0.836428 & -2.661014 \\
\hline 28 & 6 & 0 & -3.389798 & -3.756515 & -3.018103 \\
\hline 29 & 1 & 0 & -4.432919 & -4.782108 & -1.436920 \\
\hline 30 & 1 & 0 & -2.258184 & -2.529127 & -4.383027 \\
\hline 31 & 1 & 0 & -3.625032 & -4.510169 & -3.767871 \\
\hline 32 & 6 & 0 & -2.568774 & -1.241806 & 1.765796 \\
\hline 33 & 6 & 0 & -1.424534 & -1.616580 & 2.474961 \\
\hline 34 & 6 & 0 & -3.830017 & -1.451320 & 2.338166 \\
\hline 35 & 6 & 0 & -1.540474 & -2.198213 & 3.733611 \\
\hline 36 & 1 & 0 & -0.441040 & -1.466582 & 2.027289 \\
\hline 37 & 6 & 0 & -3.940955 & -2.033953 & 3.594975 \\
\hline 38 & 1 & 0 & -4.728181 & -1.147320 & 1.799638 \\
\hline 39 & 6 & 0 & -2.795703 & -2.406872 & 4.294286 \\
\hline 40 & 1 & 0 & -0.642603 & -2.487735 & 4.276629 \\
\hline 41 & 1 & 0 & -4.925406 & -2.192179 & 4.032575 \\
\hline 42 & 1 & 0 & -2.885026 & -2.859323 & 5.280792 \\
\hline 43 & 6 & 0 & -3.831713 & 0.609177 & -0.035827 \\
\hline 44 & 6 & 0 & -4.632810 & 0.628130 & -1.178660 \\
\hline 45 & 6 & 0 & -4.049457 & 1.562270 & 0.965946 \\
\hline 46 & 6 & 0 & -5.629530 & 1.590483 & -1.321861 \\
\hline 47 & 1 & 0 & -4.478551 & -0.110421 & -1.964971 \\
\hline 48 & 6 & 0 & -5.043075 & 2.519695 & 0.820739 \\
\hline 49 & 1 & 0 & -3.424812 & 1.566763 & 1.859366 \\
\hline 50 & 6 & 0 & -5.832638 & 2.539191 & -0.327033 \\
\hline 51 & 1 & 0 & -6.249095 & 1.594309 & -2.217381 \\
\hline 52 & 1 & 0 & -5.189330 & 3.265586 & 1.600440 \\
\hline 53 & 1 & 0 & -6.606555 & 3.296226 & -0.444182 \\
\hline 54 & 6 & 0 & 4.956823 & 2.690836 & 0.584328 \\
\hline 55 & 1 & 0 & 5.580471 & 3.407382 & 0.041621 \\
\hline 56 & 1 & 0 & 5.490914 & 1.720571 & 0.642314 \\
\hline 57 & 1 & 0 & 4.776920 & 3.033718 & 1.610470 \\
\hline 58 & 1 & 0 & 1.118736 & 2.636301 & -2.017604 \\
\hline 59 & 1 & 0 & -2.742484 & 5.868167 & 0.305492 \\
\hline 60 & 7 & 0 & 6.354572 & -0.538464 & 0.543769 \\
\hline 61 & 6 & 0 & 7.796462 & -0.350694 & 0.441007 \\
\hline 62 & 6 & 0 & 8.205757 & 0.514741 & -0.735295 \\
\hline 63 & 1 & 0 & 8.324116 & -1.328867 & 0.387550 \\
\hline 64 & 1 & 0 & 8.134518 & 0.131095 & 1.371008 \\
\hline 65 & 1 & 0 & 9.291545 & 0.672012 & -0.734349 \\
\hline
\end{tabular}




$\begin{array}{rrrrrr}66 & 1 & 0 & 7.942144 & 0.058468 & -1.698201 \\ 67 & 1 & 0 & 7.715894 & 1.496185 & -0.684697 \\ 68 & 6 & 0 & 5.990483 & -1.143829 & 1.820928 \\ 69 & 6 & 0 & 4.513812 & -1.005625 & 2.135227 \\ 70 & 1 & 0 & 6.571765 & -0.637420 & 2.606560 \\ 71 & 1 & 0 & 6.290858 & -2.213643 & 1.854138 \\ 72 & 1 & 0 & 4.279566 & -1.476980 & 3.098127 \\ 73 & 1 & 0 & 4.238464 & 0.056051 & 2.197530 \\ 74 & 1 & 0 & 3.868231 & -1.468700 & 1.375453 \\ 75 & 6 & 0 & 5.807047 & -1.289892 & -0.598691 \\ 76 & 6 & 0 & 4.891773 & -0.452542 & -1.469723 \\ 77 & 1 & 0 & 5.249642 & -2.164495 & -0.227666 \\ 78 & 1 & 0 & 6.635208 & -1.707036 & -1.197051 \\ 79 & 1 & 0 & 4.527118 & -1.030345 & -2.329824 \\ 80 & 1 & 0 & 4.012279 & -0.137698 & -0.890610 \\ 81 & 1 & 0 & 5.400025 & 0.446746 & -1.843365\end{array}$

$\begin{array}{lc}\text { Zero-point correction= } & 0.669690 \text { (Hartree/Particle) } \\ \text { Thermal correction to Energy= } & 0.712292 \\ \text { Thermal correction to Enthalpy= } & 0.713236 \\ \text { Thermal correction to Gibbs Free Energy= } & 0.589691 \\ \text { Sum of electronic and zero-point Energies }= & -1965.958207 \\ \text { Sum of electronic and thermal Energies }= & -1965.915605 \\ \text { Sum of electronic and thermal Enthalpies }= & -1965.914661 \\ \text { Sum of electronic and thermal Free Energies }= & -1966.038206\end{array}$

Sum of electronic and thermal Free Energies $=\quad-1966.038206$

M06 /6-311++G(d,p)-SDD/SMD//M06/6-31G(d)-LANL2DZ energy in toluene solvent $=-1968.2961786$

TS6"

\begin{tabular}{|c|c|c|c|c|c|}
\hline \multirow{2}{*}{$\begin{array}{l}\text { Center } \\
\text { Number }\end{array}$} & \multirow{2}{*}{$\begin{array}{l}\text { Atomic } \\
\text { Number }\end{array}$} & \multirow{2}{*}{$\begin{array}{l}\text { Atomic } \\
\text { Type }\end{array}$} & \multicolumn{3}{|c|}{ Coordinates (Angstroms) } \\
\hline & & & $X$ & $\mathrm{Y}$ & \\
\hline 1 & 6 & 0 & 1.562987 & 4.780193 & 1.531900 \\
\hline 2 & 6 & 0 & 1.173035 & 3.716722 & 2.341056 \\
\hline 3 & 6 & 0 & 0.710898 & 2.532601 & 1.768561 \\
\hline 4 & 6 & 0 & 0.638838 & 2.396280 & 0.380310 \\
\hline 5 & 6 & 0 & 1.011385 & 3.472890 & -0.425834 \\
\hline 6 & 6 & 0 & 1.480566 & 4.655354 & 0.147956 \\
\hline 7 & 1 & 0 & 1.231540 & 3.804502 & 3.425987 \\
\hline 8 & 1 & 0 & 0.427180 & 1.702047 & 2.419963 \\
\hline 9 & 1 & 0 & 0.939619 & 3.394161 & -1.512183 \\
\hline 10 & 1 & 0 & 1.775765 & 5.485898 & -0.493642 \\
\hline 11 & 53 & 0 & -1.027232 & -1.595411 & -1.749681 \\
\hline 12 & 46 & 0 & -0.031784 & 0.671875 & -0.496892 \\
\hline 13 & 15 & 0 & 2.004357 & -0.428462 & 0.061216 \\
\hline 14 & 6 & 0 & -2.380035 & 2.215508 & -2.093780 \\
\hline 15 & 1 & 0 & -1.993147 & 2.269192 & -3.108098 \\
\hline 16 & 6 & 0 & -1.768992 & 1.675836 & -1.024539 \\
\hline 17 & 6 & 0 & -2.382268 & 1.612579 & 0.208645 \\
\hline 18 & 1 & 0 & -2.913985 & 0.688201 & 0.524323 \\
\hline 19 & 6 & 0 & -3.720222 & 2.676261 & -1.742274 \\
\hline 20 & 8 & 0 & -4.125202 & 2.576581 & -0.575000 \\
\hline 21 & 6 & 0 & 1.682109 & -2.046414 & 0.844996 \\
\hline 22 & 6 & 0 & 2.230272 & -3.237153 & 0.370426 \\
\hline 23 & 6 & 0 & 0.792252 & -2.084523 & 1.924682 \\
\hline 24 & 6 & 0 & 1.904055 & -4.447473 & 0.975995 \\
\hline 25 & 1 & 0 & 2.898260 & -3.225493 & -0.489517 \\
\hline 26 & 6 & 0 & 0.482405 & -3.291042 & 2.537802 \\
\hline 27 & 1 & 0 & 0.328401 & -1.161164 & 2.279221 \\
\hline 28 & 6 & 0 & 1.036377 & -4.476816 & 2.060973 \\
\hline 29 & 1 & 0 & 2.327219 & -5.373385 & 0.590171 \\
\hline 30 & 1 & 0 & -0.202804 & -3.307525 & 3.384680 \\
\hline 31 & 1 & 0 & 0.782500 & -5.424855 & 2.532189 \\
\hline 32 & 6 & 0 & 3.109504 & -0.759812 & -1.355882 \\
\hline 33 & 6 & 0 & 2.626370 & -0.658307 & -2.661796 \\
\hline 34 & 6 & 0 & 4.446092 & -1.120309 & -1.141672 \\
\hline 35 & 6 & 0 & 3.469073 & -0.916070 & -3.739676 \\
\hline 36 & 1 & 0 & 1.581367 & -0.395411 & -2.829242 \\
\hline 37 & 6 & 0 & 5.282364 & -1.380332 & -2.219522 \\
\hline 38 & 1 & 0 & 4.831913 & -1.195196 & -0.124243 \\
\hline 39 & 6 & 0 & 4.793845 & -1.276345 & -3.520241 \\
\hline 40 & 1 & 0 & 3.082929 & -0.836659 & -4.754252 \\
\hline 41 & 1 & 0 & 6.319573 & -1.661472 & -2.044507 \\
\hline 42 & 1 & 0 & 5.450883 & -1.478074 & -4.364862 \\
\hline 43 & 6 & 0 & 3.122589 & 0.436774 & 1.227450 \\
\hline 44 & 6 & 0 & 3.339624 & 0.004972 & 2.536183 \\
\hline 45 & 6 & 0 & 3.724435 & 1.622848 & 0.790720 \\
\hline 46 & 6 & 0 & 4.138716 & 0.753591 & 3.398003 \\
\hline 47 & 1 & 0 & 2.888141 & -0.921510 & 2.888951 \\
\hline 48 & 6 & 0 & 4.522826 & 2.363877 & 1.649369 \\
\hline 49 & 1 & 0 & 3.547372 & 1.980674 & -0.224177 \\
\hline 50 & 6 & 0 & 4.729018 & 1.931728 & 2.958302 \\
\hline 51 & 1 & 0 & 4.302353 & 0.406114 & 4.416999 \\
\hline 52 & 1 & 0 & 4.976292 & 3.289516 & 1.298744 \\
\hline 53 & 1 & 0 & 5.351117 & 2.516927 & 3.633777 \\
\hline 54 & 6 & 0 & -4.603249 & 3.234173 & -2.814724 \\
\hline 55 & 1 & 0 & -5.535485 & 3.618283 & -2.392931 \\
\hline 56 & 1 & 0 & -4.831786 & 2.443311 & -3.542024 \\
\hline 57 & 1 & 0 & -4.082920 & 4.030022 & -3.362626 \\
\hline 58 & 1 & 0 & -2.155347 & 2.360773 & 0.971657 \\
\hline 59 & 1 & 0 & 1.926670 & 5.704958 & 1.977896 \\
\hline 60 & 7 & 0 & -3.945682 & -0.803774 & 1.314243 \\
\hline 61 & 6 & 0 & -4.481194 & -1.701741 & 0.301087 \\
\hline
\end{tabular}




$\begin{array}{rrrrrr}62 & 6 & 0 & -5.075592 & -0.956082 & -0.879614 \\ 63 & 1 & 0 & -5.227286 & -2.408512 & 0.716743 \\ 64 & 1 & 0 & -3.639301 & -2.310226 & -0.059267 \\ 65 & 1 & 0 & -5.423726 & -1.667568 & -1.638500 \\ 66 & 1 & 0 & -5.929860 & -0.326689 & -0.595141 \\ 67 & 1 & 0 & -4.312471 & -0.315346 & -1.343525 \\ 68 & 6 & 0 & -2.902410 & -1.375170 & 2.150398 \\ 69 & 6 & 0 & -3.336343 & -2.394862 & 3.197065 \\ 70 & 1 & 0 & -2.377945 & -0.537759 & 2.649003 \\ 71 & 1 & 0 & -2.164623 & -1.832667 & 1.469388 \\ 72 & 1 & 0 & -2.460087 & -2.844224 & 3.684201 \\ 73 & 1 & 0 & -3.950688 & -1.939271 & 3.984914 \\ 74 & 1 & 0 & -3.914961 & -3.209890 & 2.741619 \\ 75 & 6 & 0 & -4.911175 & 0.064581 & 1.978749 \\ 76 & 6 & 0 & -6.166011 & -0.591880 & 2.545063 \\ 77 & 1 & 0 & -5.215605 & 0.851472 & 1.267587 \\ 78 & 1 & 0 & -4.369387 & 0.586459 & 2.784241 \\ 79 & 1 & 0 & -6.795074 & 0.162726 & 3.034359 \\ 80 & 1 & 0 & -6.769644 & -1.056161 & 1.753992 \\ 81 & 1 & 0 & -5.934399 & -1.369547 & 3.283481 \\ ---------------------------------------- & & \end{array}$

Zero-point correction $=0.669034$ (Hartree/Particle)

Thermal correction to Energy= 0.712010

Thermal correction to Enthalpy=

Thermal correction to Gibbs Free Energy=

Sum of electronic and zero-point Energies

Sum of electronic and thermal Energies=

0.712954

0.588822

Sum of electronic and thermal Enthalpies $=\quad-1965.91958$

Sum of electronic and thermal Free Energies= $\quad-1966.042769$

M06 /6-311++G(d,p)-SDD/SMD//M06/6-31G(d)-LANL2DZ energy in toluene solvent = -1968.2988243

TS6-cis-1

\begin{tabular}{|c|c|c|c|c|c|}
\hline \multirow{2}{*}{$\begin{array}{l}\text { Center } \\
\text { Number }\end{array}$} & \multirow{2}{*}{$\begin{array}{l}\text { Atomic } \\
\text { Number }\end{array}$} & \multirow{2}{*}{$\begin{array}{l}\text { Atomic } \\
\text { Type }\end{array}$} & \multicolumn{3}{|c|}{ Coordinates (Angstroms) } \\
\hline & & & $\mathrm{X}$ & $\mathrm{Y}$ & 2 \\
\hline 1 & 6 & 0 & -5.837587 & -0.527990 & 0.012176 \\
\hline 2 & 6 & 0 & -5.177307 & -0.771360 & -1.188360 \\
\hline 3 & 6 & 0 & -3.783858 & -0.752824 & -1.242511 \\
\hline 4 & 6 & 0 & -3.045876 & -0.482369 & -0.091554 \\
\hline 5 & 6 & 0 & -3.705253 & -0.236292 & 1.110322 \\
\hline 6 & 6 & 0 & -5.098788 & -0.264279 & 1.161233 \\
\hline 7 & 1 & 0 & -5.747343 & -0.991656 & -2.090998 \\
\hline 8 & 1 & 0 & -3.276329 & -0.978315 & -2.181509 \\
\hline 9 & 1 & 0 & -3.135172 & -0.029072 & 2.018233 \\
\hline 10 & 1 & 0 & -5.606678 & -0.079611 & 2.108020 \\
\hline 11 & 53 & 0 & -1.022143 & -3.047494 & -0.035668 \\
\hline 12 & 46 & 0 & -1.000637 & -0.361536 & -0.146528 \\
\hline 13 & 15 & 0 & 1.458053 & -0.009865 & -0.068031 \\
\hline 14 & 6 & 0 & -1.247365 & 2.654025 & 0.939147 \\
\hline 15 & 1 & 0 & -1.051383 & 2.549328 & 2.004290 \\
\hline 16 & 6 & 0 & -1.254724 & 1.675617 & 0.012783 \\
\hline 17 & 6 & 0 & -1.530100 & 1.998444 & -1.309035 \\
\hline 18 & 1 & 0 & -0.720725 & 2.122851 & -2.032535 \\
\hline 19 & 6 & 0 & -1.530101 & 3.944806 & 0.322935 \\
\hline 20 & 8 & 0 & -1.738671 & 4.004744 & -0.899602 \\
\hline 21 & 6 & 0 & 1.795889 & 1.452561 & -1.117984 \\
\hline 22 & 6 & 0 & 1.943642 & 2.743454 & -0.606162 \\
\hline 23 & 6 & 0 & 1.709698 & 1.281102 & -2.507429 \\
\hline 24 & 6 & 0 & 2.011934 & 3.839186 & -1.464407 \\
\hline 25 & 1 & 0 & 1.993630 & 2.895544 & 0.472145 \\
\hline 26 & 6 & 0 & 1.794974 & 2.373556 & -3.362517 \\
\hline 27 & 1 & 0 & 1.568983 & 0.280156 & -2.920800 \\
\hline 28 & 6 & 0 & 1.941031 & 3.657998 & -2.841084 \\
\hline 29 & 1 & 0 & 2.121107 & 4.840856 & -1.050857 \\
\hline 30 & 1 & 0 & 1.735466 & 2.223607 & -4.439219 \\
\hline 31 & 1 & 0 & 1.993042 & 4.516010 & -3.508713 \\
\hline 32 & 6 & 0 & 1.934414 & 0.478397 & 1.631675 \\
\hline 33 & 6 & 0 & 0.995105 & 0.338143 & 2.655793 \\
\hline 34 & 6 & 0 & 3.223548 & 0.927718 & 1.939794 \\
\hline 35 & 6 & 0 & 1.332889 & 0.657891 & 3.968422 \\
\hline 36 & 1 & 0 & -0.003344 & -0.038110 & 2.417254 \\
\hline 37 & 6 & 0 & 3.557311 & 1.252825 & 3.248360 \\
\hline 38 & 1 & 0 & 3.969964 & 1.019321 & 1.149233 \\
\hline 39 & 6 & 0 & 2.611502 & 1.118309 & 4.263146 \\
\hline 40 & 1 & 0 & 0.597044 & 0.536143 & 4.761495 \\
\hline 41 & 1 & 0 & 4.561757 & 1.602699 & 3.481122 \\
\hline 42 & 1 & 0 & 2.877653 & 1.365225 & 5.289777 \\
\hline 43 & 6 & 0 & 2.792656 & -1.186917 & -0.508443 \\
\hline 44 & 6 & 0 & 3.886256 & -0.845336 & -1.309306 \\
\hline 45 & 6 & 0 & 2.726135 & -2.467556 & 0.051449 \\
\hline 46 & 6 & 0 & 4.890996 & -1.775525 & -1.555908 \\
\hline 47 & 1 & 0 & 3.959023 & 0.152117 & -1.741638 \\
\hline 48 & 6 & 0 & 3.734040 & -3.391841 & -0.195499 \\
\hline 49 & 1 & 0 & 1.872284 & -2.743050 & 0.670797 \\
\hline 50 & 6 & 0 & 4.815219 & -3.049294 & -1.001836 \\
\hline 51 & 1 & 0 & 5.738752 & -1.500097 & -2.181482 \\
\hline 52 & 1 & 0 & 3.667279 & -4.387471 & 0.239246 \\
\hline 53 & 1 & 0 & 5.600989 & -3.777134 & -1.198014 \\
\hline 54 & 6 & 0 & -1.548864 & 5.181129 & 1.162555 \\
\hline 55 & 1 & 0 & -1.797973 & 6.059958 & 0.562761 \\
\hline 56 & 1 & 0 & -0.566711 & 5.321722 & 1.633838 \\
\hline 57 & 1 & 0 & -2.277041 & 5.068915 & 1.976200 \\
\hline
\end{tabular}




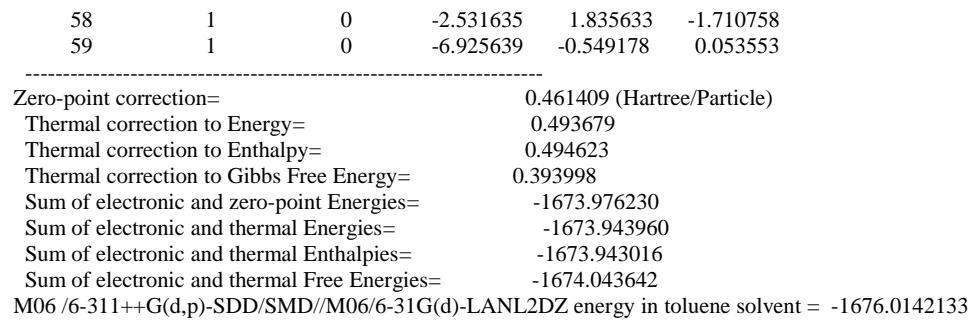

TS6-cis-2

\begin{tabular}{|c|c|c|c|c|c|}
\hline \multirow{2}{*}{$\begin{array}{l}\text { Center } \\
\text { Number }\end{array}$} & \multirow{2}{*}{$\begin{array}{l}\text { Atomic } \\
\text { Number }\end{array}$} & \multirow{2}{*}{$\begin{array}{l}\text { Atomic } \\
\text { Type }\end{array}$} & \multicolumn{3}{|c|}{ Coordinates (Angstroms) } \\
\hline & & & $\mathrm{X}$ & $\mathrm{Y}$ & Z \\
\hline 1 & 6 & 0 & -3.163002 & -3.701773 & 0.467537 \\
\hline 2 & 6 & 0 & -2.645835 & -3.467415 & -0.803880 \\
\hline 3 & 6 & 0 & -1.536599 & -2.641043 & -0.970280 \\
\hline 4 & 6 & 0 & -0.935118 & -2.036078 & 0.133370 \\
\hline 5 & 6 & 0 & -1.440987 & -2.290707 & 1.407200 \\
\hline 6 & 6 & 0 & -2.553907 & -3.117249 & 1.571978 \\
\hline 7 & 1 & 0 & -3.105424 & -3.933517 & -1.675861 \\
\hline 8 & 1 & 0 & -1.137413 & -2.474233 & -1.972082 \\
\hline 9 & 1 & 0 & -0.972375 & -1.851109 & 2.288879 \\
\hline 10 & 1 & 0 & -2.940769 & -3.304665 & 2.573931 \\
\hline 11 & 53 & 0 & 2.247266 & -2.920003 & -0.148080 \\
\hline 12 & 46 & 0 & 0.650113 & -0.751814 & -0.148757 \\
\hline 13 & 15 & 0 & -0.966704 & 0.961518 & -0.050144 \\
\hline 14 & 6 & 0 & 3.325612 & 0.911635 & 0.708468 \\
\hline 15 & 1 & 0 & 3.292630 & 0.805909 & 1.790721 \\
\hline 16 & 6 & 0 & 2.431044 & 0.434666 & -0.181414 \\
\hline 17 & 6 & 0 & 2.659437 & 0.648684 & -1.525825 \\
\hline 18 & 1 & 0 & 3.075087 & -0.150824 & -2.142010 \\
\hline 19 & 6 & 0 & 4.426219 & 1.577695 & 0.024341 \\
\hline 20 & 8 & 0 & 4.408816 & 1.667805 & -1.215509 \\
\hline 21 & 6 & 0 & -2.594856 & 0.508899 & -0.733038 \\
\hline 22 & 6 & 0 & -3.703763 & 0.256701 & 0.074990 \\
\hline 23 & 6 & 0 & -2.683485 & 0.267682 & -2.109813 \\
\hline 24 & 6 & 0 & -4.888967 & -0.206330 & -0.489659 \\
\hline 25 & 1 & 0 & -3.637664 & 0.395905 & 1.153240 \\
\hline 26 & 6 & 0 & -3.873577 & -0.172531 & -2.673186 \\
\hline 27 & 1 & 0 & -1.806357 & 0.411548 & -2.743963 \\
\hline 28 & 6 & 0 & -4.979585 & -0.411361 & -1.861242 \\
\hline 29 & 1 & 0 & -5.744130 & -0.413048 & 0.151349 \\
\hline 30 & 1 & 0 & -3.932135 & -0.349090 & -3.745792 \\
\hline 31 & 1 & 0 & -5.909293 & -0.771682 & -2.298404 \\
\hline 32 & 6 & 0 & -1.233584 & 1.482894 & 1.681737 \\
\hline 33 & 6 & 0 & -0.438766 & 0.925412 & 2.685672 \\
\hline 34 & 6 & 0 & -2.158080 & 2.481211 & 2.009786 \\
\hline 35 & 6 & 0 & -0.579369 & 1.344788 & 4.005667 \\
\hline 36 & 1 & 0 & 0.297076 & 0.162009 & 2.420797 \\
\hline 37 & 6 & 0 & -2.301026 & 2.893921 & 3.328257 \\
\hline 38 & 1 & 0 & -2.765281 & 2.937602 & 1.226576 \\
\hline 39 & 6 & 0 & -1.512866 & 2.323806 & 4.326637 \\
\hline 40 & 1 & 0 & 0.041763 & 0.903349 & 4.782949 \\
\hline 41 & 1 & 0 & -3.024894 & 3.667593 & 3.578523 \\
\hline 42 & 1 & 0 & -1.625195 & 2.651162 & 5.358981 \\
\hline 43 & 6 & 0 & -0.561718 & 2.572412 & -0.846658 \\
\hline 44 & 6 & 0 & -1.382013 & 3.211101 & -1.779772 \\
\hline 45 & 6 & 0 & 0.635899 & 3.191581 & -0.465393 \\
\hline 46 & 6 & 0 & -0.998436 & 4.429191 & -2.336242 \\
\hline 47 & 1 & 0 & -2.328706 & 2.761857 & -2.075182 \\
\hline 48 & 6 & 0 & 1.016266 & 4.405848 & -1.022061 \\
\hline 49 & 1 & 0 & 1.277836 & 2.714300 & 0.276884 \\
\hline 50 & 6 & 0 & 0.200646 & 5.025807 & -1.965955 \\
\hline 51 & 1 & 0 & -1.647750 & 4.913306 & -3.063967 \\
\hline 52 & 1 & 0 & 1.953491 & 4.869355 & -0.718007 \\
\hline 53 & 1 & 0 & 0.499155 & 5.975138 & -2.407251 \\
\hline 54 & 6 & 0 & 5.576720 & 2.122225 & 0.807393 \\
\hline 55 & 1 & 0 & 6.202853 & 2.769034 & 0.187517 \\
\hline 56 & 1 & 0 & 6.184769 & 1.282841 & 1.171430 \\
\hline 57 & 1 & 0 & 5.223524 & 2.667289 & 1.691133 \\
\hline 58 & 1 & 0 & 2.142217 & 1.455467 & -2.051006 \\
\hline 59 & 1 & 0 & -4.031072 & -4.347116 & 0.596203 \\
\hline
\end{tabular}

Zero-point correction=

Thermal correction to Energy=

0.460810 (Hartree/Particle)

Thermal correction to Enthalpy=

Thermal correction to Gibbs Free Energy=

Sum of electronic and zero-point Energies=

Sum of electronic and thermal Energies=
Sum of electronic and thermal Enthalpies=

0.493147

0.494091

$-1673.977012$

$-1673.944675$

-1673.944675
-1673.943730

Sum of electronic and thermal Free Energies=

$-1674.044655$

M06 /6-311++G(d,p)-SDD/SMD//M06/6-31G(d)-LANL2DZ energy in toluene solvent $=-1676.0145775$

TS6-a

Center Atomic $\quad$ Atomic $\quad$ Coordinates (Angstroms)

$\begin{array}{lllll}\text { Number } & \text { Number } & \text { Type } & \text { Coordinates (Angstroms) } & \text { Z }\end{array}$ 


\begin{tabular}{|c|c|c|c|c|c|}
\hline 1 & 6 & 0 & -3.236807 & -4.543669 & -0.072057 \\
\hline 2 & 6 & 0 & -3.169011 & -3.710638 & 1.038262 \\
\hline 3 & 6 & 0 & -2.223248 & -2.686017 & 1.091144 \\
\hline 4 & 6 & 0 & -1.359917 & -2.463763 & 0.017484 \\
\hline 5 & 6 & 0 & -1.403582 & -3.325695 & -1.078519 \\
\hline 6 & 6 & 0 & -2.343718 & -4.354715 & -1.122363 \\
\hline 7 & 1 & 0 & -3.859048 & -3.846931 & 1.871120 \\
\hline 8 & 1 & 0 & -2.208374 & -2.038319 & 1.969665 \\
\hline 9 & 1 & 0 & -0.729705 & -3.167960 & -1.920416 \\
\hline 10 & 1 & 0 & -2.378477 & -5.006680 & -1.995314 \\
\hline 11 & 53 & 0 & -0.026022 & -0.517457 & -3.108666 \\
\hline 12 & 15 & 0 & -1.943693 & 0.628577 & 0.311359 \\
\hline 13 & 15 & 0 & 1.882999 & 0.754072 & 0.163791 \\
\hline 14 & 6 & 0 & 1.733826 & 2.547078 & -0.202216 \\
\hline 15 & 6 & 0 & 1.286412 & 2.878840 & -1.486182 \\
\hline 16 & 6 & 0 & 2.164044 & 3.559577 & 0.659968 \\
\hline 17 & 6 & 0 & 1.292899 & 4.203677 & -1.904213 \\
\hline 18 & 1 & 0 & 0.940106 & 2.092072 & -2.162538 \\
\hline 19 & 6 & 0 & 2.152541 & 4.886881 & 0.238930 \\
\hline 20 & 1 & 0 & 2.525596 & 3.315909 & 1.658901 \\
\hline 21 & 6 & 0 & 1.727511 & 5.209312 & -1.045598 \\
\hline 22 & 1 & 0 & 0.949101 & 4.448206 & -2.907893 \\
\hline 23 & 1 & 0 & 2.494844 & 5.668726 & 0.915818 \\
\hline 24 & 1 & 0 & 1.731700 & 6.247183 & -1.376374 \\
\hline 25 & 6 & 0 & 2.428788 & 0.670077 & 1.917059 \\
\hline 26 & 6 & 0 & 1.609785 & 1.224594 & 2.908535 \\
\hline 27 & 6 & 0 & 3.534713 & -0.091063 & 2.312507 \\
\hline 28 & 6 & 0 & 1.890470 & 1.031503 & 4.255776 \\
\hline 29 & 1 & 0 & 0.728334 & 1.802867 & 2.625235 \\
\hline 30 & 6 & 0 & 3.811882 & -0.290128 & 3.663325 \\
\hline 31 & 1 & 0 & 4.181979 & -0.539097 & 1.558549 \\
\hline 32 & 6 & 0 & 2.992329 & 0.269660 & 4.637915 \\
\hline 33 & 1 & 0 & 1.234466 & 1.470840 & 5.006570 \\
\hline 34 & 1 & 0 & 4.673456 & -0.890095 & 3.951883 \\
\hline 35 & 1 & 0 & 3.209290 & 0.110609 & 5.692856 \\
\hline 36 & 6 & 0 & 3.436019 & 0.389947 & -0.753960 \\
\hline 37 & 6 & 0 & 3.474251 & -0.594299 & -1.739974 \\
\hline 38 & 6 & 0 & 4.589041 & 1.142379 & -0.493053 \\
\hline 39 & 6 & 0 & 4.653818 & -0.841338 & -2.439445 \\
\hline 40 & 1 & 0 & 2.563276 & -1.134637 & -1.993103 \\
\hline 41 & 6 & 0 & 5.765370 & 0.889633 & -1.185637 \\
\hline 42 & 1 & 0 & 4.565221 & 1.934243 & 0.256725 \\
\hline 43 & 6 & 0 & 5.799537 & -0.107297 & -2.159329 \\
\hline 44 & 1 & 0 & 4.665907 & -1.601972 & -3.218527 \\
\hline 45 & 1 & 0 & 6.656158 & 1.478389 & -0.972029 \\
\hline 46 & 1 & 0 & 6.720304 & -0.300098 & -2.708234 \\
\hline 47 & 6 & 0 & -1.986114 & 2.278345 & -0.502344 \\
\hline 48 & 6 & 0 & -2.413519 & 2.382077 & -1.830076 \\
\hline 49 & 6 & 0 & -1.560718 & 3.434658 & 0.161378 \\
\hline 50 & 6 & 0 & -2.457576 & 3.620141 & -2.461389 \\
\hline 51 & 1 & 0 & -2.718645 & 1.493820 & -2.379746 \\
\hline 52 & 6 & 0 & -1.625893 & 4.673441 & -0.465870 \\
\hline 53 & 1 & 0 & -1.189732 & 3.377858 & 1.184739 \\
\hline 54 & 6 & 0 & -2.082245 & 4.770745 & -1.776245 \\
\hline 55 & 1 & 0 & -2.798323 & 3.681357 & -3.493442 \\
\hline 56 & 1 & 0 & -1.310047 & 5.564855 & 0.074051 \\
\hline 57 & 1 & 0 & -2.134152 & 5.742151 & -2.266298 \\
\hline 58 & 6 & 0 & -2.209062 & 1.042893 & 2.087242 \\
\hline 59 & 6 & 0 & -1.514353 & 0.321045 & 3.059635 \\
\hline 60 & 6 & 0 & -3.110613 & 2.031887 & 2.506066 \\
\hline 61 & 6 & 0 & -1.714912 & 0.567981 & 4.416059 \\
\hline 62 & 1 & 0 & -0.792524 & -0.434165 & 2.738880 \\
\hline 63 & 6 & 0 & -3.308359 & 2.284461 & 3.858134 \\
\hline 64 & 1 & 0 & -3.660147 & 2.610774 & 1.763764 \\
\hline 65 & 6 & 0 & -2.612269 & 1.550846 & 4.816648 \\
\hline 66 & 1 & 0 & -1.162029 & -0.008022 & 5.157223 \\
\hline 67 & 1 & 0 & -4.010823 & 3.057243 & 4.166110 \\
\hline 68 & 1 & 0 & -2.770669 & 1.748907 & 5.875642 \\
\hline 69 & 6 & 0 & -3.553384 & -0.113275 & -0.195359 \\
\hline 70 & 6 & 0 & -4.680239 & -0.111622 & 0.630490 \\
\hline 71 & 6 & 0 & -3.629471 & -0.742793 & -1.443823 \\
\hline 72 & 6 & 0 & -5.866251 & -0.704701 & 0.208705 \\
\hline 73 & 1 & 0 & -4.640345 & 0.337700 & 1.621048 \\
\hline 74 & 6 & 0 & -4.817388 & -1.330736 & -1.861681 \\
\hline 75 & 1 & 0 & -2.746292 & -0.794881 & -2.085295 \\
\hline 76 & 6 & 0 & -5.940228 & -1.311327 & -1.039572 \\
\hline 77 & 1 & 0 & -6.734804 & -0.694893 & 0.865894 \\
\hline 78 & 1 & 0 & -4.857353 & -1.817881 & -2.834628 \\
\hline 79 & 1 & 0 & -6.867829 & -1.778029 & -1.367860 \\
\hline 80 & 46 & 0 & -0.003210 & -0.889823 & -0.005796 \\
\hline 81 & 1 & 0 & -3.978260 & -5.340288 & -0.117084 \\
\hline 82 & 6 & 0 & 2.158663 & -3.287877 & -0.187850 \\
\hline 83 & 1 & 0 & 2.370349 & -3.364727 & -1.252156 \\
\hline 84 & 6 & 0 & 1.315819 & -2.419025 & 0.407790 \\
\hline 85 & 6 & 0 & 1.159893 & -2.530412 & 1.795900 \\
\hline 86 & 1 & 0 & 1.655323 & -1.837422 & 2.479070 \\
\hline 87 & 6 & 0 & 2.770257 & -4.159197 & 0.798438 \\
\hline 88 & 8 & 0 & 2.467774 & -4.016587 & 2.000430 \\
\hline 89 & 6 & 0 & 3.751584 & -5.204593 & 0.383687 \\
\hline 90 & 1 & 0 & 4.112592 & -5.770665 & 1.245950 \\
\hline 91 & 1 & 0 & 4.598050 & -4.731328 & -0.131194 \\
\hline 92 & 1 & 0 & 3.284157 & -5.886973 & -0.338055 \\
\hline 93 & 1 & 0 & 0.298991 & -3.073579 & 2.189724 \\
\hline
\end{tabular}




$\begin{array}{lc}\text { Zero-point correction= } & 0.737668 \text { (Hartree/Particle) } \\ \text { Thermal correction to Energy= } & 0.786540 \\ \text { Thermal correction to Enthalpy= } & 0.787484 \\ \text { Thermal correction to Gibbs Free Energy= } & 0.653311 \\ \text { Sum of electronic and zero-point Energies }= & -2709.418452 \\ \text { Sum of electronic and thermal Energies= } & -2709.369580 \\ \text { Sum of electronic and thermal Enthalpies= } & -2709.368636 \\ \text { Sum of electronic and thermal Free Energies }= & -2709.502809\end{array}$

Sum of electronic and thermal Free Energies=

M06 /6-311++G(d,p)-SDD/SMD//M06/6-31G(d)-LANL2DZ energy in toluene solvent $=-2711.9553424$

TS7

\begin{tabular}{|c|c|c|c|c|c|}
\hline \multirow{2}{*}{$\begin{array}{l}\text { Center } \\
\text { Number }\end{array}$} & \multirow{2}{*}{$\begin{array}{l}\text { Atomic } \\
\text { Number }\end{array}$} & \multirow{2}{*}{$\begin{array}{l}\text { Atomic } \\
\text { Type }\end{array}$} & \multicolumn{3}{|c|}{ Coordinates (Angstroms) } \\
\hline & & & $\mathrm{X}$ & $\mathrm{Y}$ & Z \\
\hline 1 & 6 & 0 & 1.719449 & 4.521332 & 0.056615 \\
\hline 2 & 6 & 0 & 1.507808 & 3.847677 & -1.143593 \\
\hline 3 & 6 & 0 & 1.466811 & 2.456790 & -1.164088 \\
\hline 4 & 6 & 0 & 1.605768 & 1.719601 & 0.017409 \\
\hline 5 & 6 & 0 & 1.825518 & 2.408731 & 1.215555 \\
\hline 6 & 6 & 0 & 1.878347 & 3.798933 & 1.237065 \\
\hline 7 & 1 & 0 & 1.367459 & 4.407657 & -2.067585 \\
\hline 8 & 1 & 0 & 1.288336 & 1.939339 & -2.109680 \\
\hline 9 & 1 & 0 & 1.953053 & 1.850494 & 2.144651 \\
\hline 10 & 1 & 0 & 2.045731 & 4.320684 & 2.179175 \\
\hline 11 & 53 & 0 & 0.950283 & -2.993772 & -0.001894 \\
\hline 12 & 46 & 0 & 0.897064 & -0.260972 & 0.039607 \\
\hline 13 & 15 & 0 & -1.481340 & 0.078443 & 0.058211 \\
\hline 14 & 6 & 0 & 3.869915 & -0.031718 & 0.892639 \\
\hline 15 & 1 & 0 & 3.735150 & 0.005562 & 1.968239 \\
\hline 16 & 6 & 0 & 2.856390 & -0.011179 & -0.084671 \\
\hline 17 & 6 & 0 & 3.584660 & -0.156272 & -1.374379 \\
\hline 18 & 1 & 0 & 3.352625 & -1.129285 & -1.830349 \\
\hline 19 & 6 & 0 & 5.085904 & -0.132060 & 0.259982 \\
\hline 20 & 8 & 0 & 5.000298 & -0.161996 & -1.063956 \\
\hline 21 & 6 & 0 & -1.929303 & 1.823077 & -0.289511 \\
\hline 22 & 6 & 0 & -2.456306 & 2.228335 & -1.517412 \\
\hline 23 & 6 & 0 & -1.601526 & 2.796583 & 0.662178 \\
\hline 24 & 6 & 0 & -2.655441 & 3.580812 & -1.785941 \\
\hline 25 & 1 & 0 & -2.715653 & 1.483979 & -2.270307 \\
\hline 26 & 6 & 0 & -1.808371 & 4.142972 & 0.395975 \\
\hline 27 & 1 & 0 & -1.174433 & 2.493903 & 1.619612 \\
\hline 28 & 6 & 0 & -2.333615 & 4.539377 & -0.831994 \\
\hline 29 & 1 & 0 & -3.069555 & 3.883812 & -2.746629 \\
\hline 30 & 1 & 0 & -1.548266 & 4.887777 & 1.147060 \\
\hline 31 & 1 & 0 & -2.492017 & 5.595947 & -1.043227 \\
\hline 32 & 6 & 0 & -2.419283 & -0.841705 & -1.215647 \\
\hline 33 & 6 & 0 & -3.790388 & -1.088386 & -1.108895 \\
\hline 34 & 6 & 0 & -1.738381 & -1.244942 & -2.366785 \\
\hline 35 & 6 & 0 & -4.469032 & -1.725841 & -2.141326 \\
\hline 36 & 1 & 0 & -4.329887 & -0.787865 & -0.210250 \\
\hline 37 & 6 & 0 & -2.419877 & -1.875236 & -3.402445 \\
\hline 38 & 1 & 0 & -0.663038 & -1.073839 & -2.436984 \\
\hline 39 & 6 & 0 & -3.785375 & -2.116920 & -3.289444 \\
\hline 40 & 1 & 0 & -5.536329 & -1.920680 & -2.047884 \\
\hline 41 & 1 & 0 & -1.879123 & -2.190480 & -4.293167 \\
\hline 42 & 1 & 0 & -4.318029 & -2.619042 & -4.095761 \\
\hline 43 & 6 & 0 & -2.349038 & -0.285198 & 1.629779 \\
\hline 44 & 6 & 0 & -3.505201 & 0.392007 & 2.034322 \\
\hline 45 & 6 & 0 & -1.835654 & -1.305210 & 2.437041 \\
\hline 46 & 6 & 0 & -4.139546 & 0.049261 & 3.223409 \\
\hline 47 & 1 & 0 & -3.906415 & 1.197890 & 1.419089 \\
\hline 48 & 6 & 0 & -2.475380 & -1.647072 & 3.624257 \\
\hline 49 & 1 & 0 & -0.934468 & -1.836645 & 2.125376 \\
\hline 50 & 6 & 0 & -3.625171 & -0.970997 & 4.019074 \\
\hline 51 & 1 & 0 & -5.037514 & 0.583435 & 3.530571 \\
\hline 52 & 1 & 0 & -2.068711 & -2.444369 & 4.244045 \\
\hline 53 & 1 & 0 & -4.122201 & -1.236976 & 4.950898 \\
\hline 54 & 6 & 0 & 6.446875 & -0.224471 & 0.826111 \\
\hline 55 & 1 & 0 & 6.901951 & -1.186037 & 0.555939 \\
\hline 56 & 1 & 0 & 6.426662 & -0.133969 & 1.915641 \\
\hline 57 & 1 & 0 & 3.401484 & 0.639892 & -2.102638 \\
\hline 58 & 1 & 0 & 7.085288 & 0.563577 & 0.407587 \\
\hline 59 & 1 & 0 & 1.762304 & 5.609484 & 0.071825 \\
\hline
\end{tabular}

Zero-point correction=

0.463078 (Hartree/Particle)

Thermal correction to Energy=

Thermal correction to Enthalpy=

Thermal correction to Gibbs Free Energy=

Sum of electronic and zero-point Energies $=$

Sum of electronic and thermal Energies=

Sum of electronic and thermal Enthalpies=

Sum of electronic and thermal Free Energies=

0.494929

0.495873

0.394704

$-1674.003203$

$-1673.971353$

$-1673.970408$

M06 /6-311++G(d,p)-SDD/SMD//M06/6-31G(d)-LANL2DZ energy in toluene solvent $=-1676.0388618$

3a

\begin{tabular}{cccccc} 
Center & Atomic & Atomic & \multicolumn{3}{c}{ Coordinates (Angstroms) } \\
Number & Number & Type & X & Y & Z \\
- \hdashline 1 & 6 & 0 & -3.710496 & -0.233750 & 0.039255 \\
2 & 6 & 0 & -2.900059 & -1.337478 & -0.205601
\end{tabular}




$\begin{array}{rrrrrr}3 & 6 & 0 & -1.518212 & -1.198757 & -0.230583 \\ 4 & 6 & 0 & -0.918351 & 0.045104 & -0.008672 \\ 5 & 6 & 0 & -1.744649 & 1.146420 & 0.244036 \\ 6 & 6 & 0 & -3.126035 & 1.009664 & 0.262168 \\ 7 & 1 & 0 & -3.347421 & -2.314522 & -0.381957 \\ 8 & 1 & 0 & -0.889989 & -2.062963 & -0.444624 \\ 9 & 1 & 0 & -1.291029 & 2.116024 & 0.449422 \\ 10 & 1 & 0 & -3.750681 & 1.878263 & 0.464747 \\ 11 & 6 & 0 & 1.518749 & -0.832243 & 0.217046 \\ 12 & 1 & 0 & 1.332278 & -1.860128 & 0.508165 \\ 13 & 6 & 0 & 0.537596 & 0.184525 & -0.041453 \\ 14 & 6 & 0 & 1.258104 & 1.303917 & -0.334040 \\ 15 & 6 & 0 & 2.738432 & -0.255006 & 0.071606 \\ 16 & 8 & 0 & 2.590425 & 1.054488 & -0.270415 \\ 17 & 6 & 0 & 4.124764 & -0.749916 & 0.215295 \\ 18 & 1 & 0 & 4.671721 & -0.199335 & 0.992057 \\ 19 & 1 & 0 & 4.689736 & -0.643614 & -0.720178 \\ 20 & 1 & 0 & 4.116025 & -1.809699 & 0.490436 \\ 21 & 1 & 0 & -4.793400 & -0.342205 & 0.060504 \\ 22 & 1 & 0 & 0.980305 & 2.307393 & -0.629602\end{array}$

0.188536

Ther correction to Energy=

Thermal correction to Gibbs Free Energy=

Sum of electronic and zero-point Energies

Sum of electronic and thermal Energies=

0.142651

Sum of electronic and thermal Enthalpies=

$-499.855676$

$-499.845748$

$-499.844803$

M06 /6-311++G(d,p)-SDD/SMD//M06/6-31G(d)-LANL2DZ energy in toluene solvent $=-500.1658652$

$\mathbf{E t}_{3} \mathbf{N}$

\begin{tabular}{|c|c|c|c|c|c|}
\hline \multirow{2}{*}{$\begin{array}{l}\text { Center } \\
\text { Number }\end{array}$} & \multirow{2}{*}{$\begin{array}{l}\text { Atomic } \\
\text { Number }\end{array}$} & \multirow{2}{*}{$\begin{array}{l}\text { Atomic } \\
\text { Type }\end{array}$} & \multicolumn{3}{|c|}{ Coordinates (Angstroms) } \\
\hline & & & $X$ & $\mathrm{Y}$ & \\
\hline 1 & 7 & 0 & 0.001111 & -0.000674 & -0.025532 \\
\hline 2 & 6 & 0 & -0.100101 & 1.384813 & 0.422082 \\
\hline 3 & 6 & 0 & -1.068719 & 2.205376 & -0.408106 \\
\hline 4 & 1 & 0 & 0.894946 & 1.841484 & 0.346534 \\
\hline 5 & 1 & 0 & -0.372027 & 1.430130 & 1.499018 \\
\hline 6 & 1 & 0 & -1.081878 & 3.247669 & -0.071146 \\
\hline 7 & 1 & 0 & -0.773032 & 2.182885 & -1.462191 \\
\hline 8 & 1 & 0 & -2.096141 & 1.829452 & -0.342584 \\
\hline 9 & 6 & 0 & -1.146559 & -0.780670 & 0.426339 \\
\hline 10 & 6 & 0 & -1.382015 & -2.024278 & -0.409887 \\
\hline 11 & 1 & 0 & -2.039215 & -0.145401 & 0.361954 \\
\hline 12 & 1 & 0 & -1.041829 & -1.045494 & 1.500864 \\
\hline 13 & 1 & 0 & -2.274301 & -2.559695 & -0.067395 \\
\hline 14 & 1 & 0 & -1.522489 & -1.749301 & -1.460493 \\
\hline 15 & 1 & 0 & -0.541902 & -2.726332 & -0.359996 \\
\hline 16 & 6 & 0 & 1.251423 & -0.604528 & 0.423616 \\
\hline 17 & 6 & 0 & 2.445597 & -0.180409 & -0.409862 \\
\hline 18 & 1 & 0 & 1.148858 & -1.694815 & 0.353682 \\
\hline 19 & 1 & 0 & 1.427962 & -0.386769 & 1.499310 \\
\hline 20 & 1 & 0 & 3.355916 & -0.686210 & -0.069779 \\
\hline 21 & 1 & 0 & 2.277768 & -0.432738 & -1.462103 \\
\hline 22 & 1 & 0 & 2.631837 & 0.898034 & -0.352044 \\
\hline
\end{tabular}

Zero-point correction=

0.205004 (Hartree/Particle)

Thermal correction to Energy=

Thermal correction to Enthalpy=

Thermal correction to Gibbs Free Energy=

Sum of electronic and zero-point Energies $=$

Sum of electronic and thermal Energies=

Sum of electronic and thermal Enthalpies=

Sum of electronic and thermal Free Energies=

0.214384

0.215328

0.171213

291.981656
-291.972276

291.971332

M06 /6-311++G(d,p)-SDD/SMD//M06/6-31G(d)-LANL2DZ energy in toluene solvent $=-292.2636923$

\section{$\mathrm{Et}_{3} \mathrm{~N}^{+} \mathrm{HI}$}

\begin{tabular}{rrrrrr} 
Center & Atomic & Atomic & \multicolumn{3}{c}{ Coordinates (Angstroms) } \\
Number & Number & Type & X & Y \\
\hline 1 & 7 & 0 & -1.516724 & 0.000100 & -0.330356 \\
2 & 6 & 0 & -1.635306 & 1.234067 & -1.170107 \\
3 & 6 & 0 & -1.293246 & 2.490230 & -0.401055 \\
4 & 1 & 0 & -2.653669 & 1.247455 & -1.584727 \\
5 & 1 & 0 & -0.920802 & 1.096961 & -1.991651 \\
6 & 1 & 0 & -1.332176 & 3.347704 & -1.081752 \\
7 & 1 & 0 & -1.990553 & 2.692149 & 0.421462 \\
8 & 1 & 0 & -0.272837 & 2.419807 & -0.000706 \\
9 & 6 & 0 & -1.635798 & -1.233838 & -1.170191 \\
10 & 6 & 0 & -1.293558 & -2.490109 & -0.401352 \\
11 & 1 & 0 & -0.921540 & -1.096786 & -1.991944 \\
12 & 1 & 0 & -2.654304 & -1.247054 & -1.584439 \\
13 & 1 & 0 & -1.332740 & -3.347547 & -1.082097 \\
14 & 1 & 0 & -0.273000 & -2.419771 & -0.001341 \\
15 & 1 & 0 & -1.990577 & -2.692034 & 0.421385 \\
16 & 6 & 0 & -2.430071 & 0.000263 & 0.862725 \\
17 & 6 & 0 & -1.653519 & -0.000315 & 2.161255 \\
18 & 1 & 0 & -3.080391 & -0.878497 & 0.784000 \\
19 & 1 & 0 & -3.079669 & 0.879581 & 0.784283 \\
& 1 & 0 & & &
\end{tabular}




$\begin{array}{rrrrrr}20 & 1 & 0 & -2.347735 & -0.000136 & 3.009166 \\ 21 & 1 & 0 & -1.006155 & -0.881960 & 2.238932 \\ 22 & 1 & 0 & -1.005316 & 0.880685 & 2.239322 \\ 23 & 53 & 0 & 1.803990 & -0.000056 & 0.046315 \\ 24 & 1 & 0 & -0.483932 & -0.000101 & -0.009740\end{array}$

Thermal correction to Energy $=\quad 0.231141$

Thermal correction to Enthalpy=

Thermal correction to Gibbs Free Energy $=\quad 0.181281$

Sum of electronic and zero-point Energies $=\quad-303.931484$

Sum of electronic and thermal Energies $=\quad-303.920101$

$\begin{array}{ll}\text { Sum of electronic and thermal Enthalpies }= & -303.919157 \\ \text { Sum of electronic and thermal Free Energies }= & -303.969962\end{array}$

M06 /6-311++G(d,p)-SDD/SMD//M06/6-31G(d)-LANL2DZ energy in toluene solvent $=-304.2997452$

INT1

\begin{tabular}{|c|c|c|c|c|c|}
\hline \multirow{2}{*}{$\begin{array}{l}\text { Center } \\
\text { Number }\end{array}$} & \multirow{2}{*}{$\begin{array}{l}\text { Atomic } \\
\text { Number }\end{array}$} & \multirow{2}{*}{$\begin{array}{l}\text { Atomic } \\
\text { Type }\end{array}$} & \multicolumn{3}{|c|}{ Coordinates (Angstroms) } \\
\hline & & & $\mathrm{X}$ & $\mathrm{Y}$ & \\
\hline 1 & 15 & 0 & -2.020616 & -0.362806 & -0.257019 \\
\hline 2 & 46 & 0 & 0.280254 & -1.043414 & -0.658741 \\
\hline 3 & 15 & 0 & 1.613389 & 0.871319 & -0.112054 \\
\hline 4 & 6 & 0 & -2.533412 & -1.243798 & 1.269084 \\
\hline 5 & 6 & 0 & -1.622422 & -1.255691 & 2.333065 \\
\hline 6 & 6 & 0 & -3.773958 & -1.863903 & 1.428820 \\
\hline 7 & 6 & 0 & -1.956992 & -1.855945 & 3.540309 \\
\hline 8 & 1 & 0 & -0.644099 & -0.783594 & 2.214182 \\
\hline 9 & 6 & 0 & -4.098897 & -2.482528 & 2.633764 \\
\hline 10 & 1 & 0 & -4.492037 & -1.866938 & 0.608903 \\
\hline 11 & 6 & 0 & -3.195717 & -2.474654 & 3.691533 \\
\hline 12 & 1 & 0 & -1.240694 & -1.844670 & 4.360901 \\
\hline 13 & 1 & 0 & -5.066672 & -2.969385 & 2.746011 \\
\hline 14 & 1 & 0 & -3.454853 & -2.956101 & 4.633306 \\
\hline 15 & 6 & 0 & 1.304686 & 1.144419 & 1.672365 \\
\hline 16 & 6 & 0 & 1.850008 & 0.219920 & 2.575239 \\
\hline 17 & 6 & 0 & 0.379208 & 2.080015 & 2.143984 \\
\hline 18 & 6 & 0 & 1.503701 & 0.254904 & 3.920360 \\
\hline 19 & 1 & 0 & 2.540498 & -0.543940 & 2.212575 \\
\hline 20 & 6 & 0 & 0.028487 & 2.107743 & 3.491722 \\
\hline 21 & 1 & 0 & -0.086728 & 2.785266 & 1.455332 \\
\hline 22 & 6 & 0 & 0.591256 & 1.201769 & 4.383296 \\
\hline 23 & 1 & 0 & 1.940985 & -0.466401 & 4.609358 \\
\hline 24 & 1 & 0 & -0.697271 & 2.841491 & 3.840016 \\
\hline 25 & 1 & 0 & 0.313840 & 1.224648 & 5.435958 \\
\hline 26 & 6 & 0 & -3.322267 & -0.800982 & -1.474488 \\
\hline 27 & 6 & 0 & -4.562233 & -0.155533 & -1.530804 \\
\hline 28 & 6 & 0 & -3.058197 & -1.845683 & -2.363910 \\
\hline 29 & 6 & 0 & -5.518273 & -0.553870 & -2.459156 \\
\hline 30 & 1 & 0 & -4.777890 & 0.663427 & -0.843558 \\
\hline 31 & 6 & 0 & -4.017466 & -2.248146 & -3.287406 \\
\hline 32 & 1 & 0 & -2.085017 & -2.337153 & -2.326521 \\
\hline 33 & 6 & 0 & -5.247820 & -1.600345 & -3.336971 \\
\hline 34 & 1 & 0 & -6.479721 & -0.043782 & -2.498525 \\
\hline 35 & 1 & 0 & -3.799701 & -3.063692 & -3.975109 \\
\hline 36 & 1 & 0 & -5.997530 & -1.908730 & -4.064054 \\
\hline 37 & 6 & 0 & -2.457561 & 1.379044 & 0.146907 \\
\hline 38 & 6 & 0 & -2.156841 & 2.351280 & -0.814258 \\
\hline 39 & 6 & 0 & -3.019188 & 1.782460 & 1.360475 \\
\hline 40 & 6 & 0 & -2.399637 & 3.696381 & -0.568021 \\
\hline 41 & 1 & 0 & -1.714313 & 2.047445 & -1.764414 \\
\hline 42 & 6 & 0 & -3.264100 & 3.131812 & 1.608204 \\
\hline 43 & 1 & 0 & -3.261882 & 1.040912 & 2.121703 \\
\hline 44 & 6 & 0 & -2.951222 & 4.090338 & 0.649791 \\
\hline 45 & 1 & 0 & -2.152072 & 4.439567 & -1.324560 \\
\hline 46 & 1 & 0 & -3.702144 & 3.433632 & 2.559008 \\
\hline 47 & 1 & 0 & -3.139093 & 5.144330 & 0.849169 \\
\hline 48 & 6 & 0 & 3.445327 & 0.748514 & -0.229297 \\
\hline 49 & 6 & 0 & 4.329200 & 1.347346 & 0.673287 \\
\hline 50 & 6 & 0 & 3.961823 & 0.058785 & -1.329536 \\
\hline 51 & 6 & 0 & 5.702987 & 1.246534 & 0.481884 \\
\hline 52 & 1 & 0 & 3.943918 & 1.887049 & 1.538854 \\
\hline 53 & 6 & 0 & 5.336145 & -0.035633 & -1.525170 \\
\hline 54 & 1 & 0 & 3.271524 & -0.425435 & -2.023445 \\
\hline 55 & 6 & 0 & 6.208388 & 0.555634 & -0.616491 \\
\hline 56 & 1 & 0 & 6.383783 & 1.709279 & 1.195010 \\
\hline 57 & 1 & 0 & 5.725139 & -0.581326 & -2.383548 \\
\hline 58 & 1 & 0 & 7.284641 & 0.475475 & -0.761180 \\
\hline 59 & 6 & 0 & 1.353737 & 2.494617 & -0.934942 \\
\hline 60 & 6 & 0 & 1.690162 & 3.723240 & -0.358468 \\
\hline 61 & 6 & 0 & 0.865816 & 2.470773 & -2.243569 \\
\hline 62 & 6 & 0 & 1.512553 & 4.905180 & -1.068200 \\
\hline 63 & 1 & 0 & 2.086615 & 3.758439 & 0.656531 \\
\hline 64 & 6 & 0 & 0.704900 & 3.651859 & -2.962490 \\
\hline 65 & 1 & 0 & 0.602236 & 1.510898 & -2.693836 \\
\hline 66 & 6 & 0 & 1.020797 & 4.871237 & -2.371157 \\
\hline 67 & 1 & 0 & 1.767170 & 5.857681 & -0.605759 \\
\hline 68 & 1 & 0 & 0.327429 & 3.619043 & -3.983402 \\
\hline 69 & 1 & 0 & 0.889376 & 5.798314 & -2.927250 \\
\hline 70 & 6 & 0 & 2.771367 & -3.036235 & -0.472464 \\
\hline 71 & 1 & 0 & 3.518664 & -2.256126 & -0.309176 \\
\hline 72 & 6 & 0 & 1.488782 & -2.739232 & -0.677639 \\
\hline
\end{tabular}




$\begin{array}{lllrrr}73 & 6 & 0 & 0.206760 & -3.205951 & -0.880106 \\ 74 & 1 & 0 & -0.351889 & -3.656392 & -0.056186 \\ 75 & 6 & 0 & 3.244298 & -4.439148 & -0.470710 \\ 76 & 8 & 0 & 2.518457 & -5.398253 & -0.658064 \\ 77 & 6 & 0 & 4.725823 & -4.604207 & -0.211235 \\ 78 & 1 & 0 & 4.973623 & -4.243861 & 0.797188 \\ 79 & 1 & 0 & 5.312789 & -3.995581 & -0.913485 \\ 80 & 1 & 0 & -0.099276 & -3.515695 & -1.881837 \\ 81 & 1 & 0 & 5.009668 & -5.656611 & -0.301072\end{array}$

Zero-point correction=

Thermal correction to Energy=

0.643943 (Hartree/Particle)

Thermal correction to Enthalpy=

Thermal correction to Gibbs Free Energy=

Sum of electronic and zero-point Energies=

Sum of electronic and thermal Energies $=$

Sum of electronic and thermal Enthalpies=

0.687185

0.688130

Sum of electronic and thermal Free Energies=

$-2466.719180$

$-2466.675938$

$-2466.674994$

M06 /6-311++G(d,p)-SDD/SMD//M06/6-31G(d)-LANL2DZ energy in toluene solvent $=-2469.0597413$

TS1'

\begin{tabular}{|c|c|c|c|c|c|}
\hline \multirow{2}{*}{$\begin{array}{l}\text { Center } \\
\text { Number }\end{array}$} & \multirow{2}{*}{$\begin{array}{l}\text { Atomic } \\
\text { Number }\end{array}$} & \multirow{2}{*}{$\begin{array}{l}\text { Atomic } \\
\text { Type }\end{array}$} & \multicolumn{3}{|c|}{ Coordinates (Angstroms) } \\
\hline & & & $\mathrm{X}$ & $\mathrm{Y}$ & , \\
\hline 1 & 15 & 0 & 1.859434 & -0.453988 & 0.001633 \\
\hline 2 & 46 & 0 & 0.068389 & 1.147561 & 0.003624 \\
\hline 3 & 15 & 0 & -2.009643 & -0.067827 & -0.003557 \\
\hline 4 & 6 & 0 & 2.355462 & -1.042830 & 1.669587 \\
\hline 5 & 6 & 0 & 1.333449 & -1.265495 & 2.599545 \\
\hline 6 & 6 & 0 & 3.679408 & -1.294755 & 2.040883 \\
\hline 7 & 6 & 0 & 1.629104 & -1.745615 & 3.870283 \\
\hline 8 & 1 & 0 & 0.297599 & -1.059348 & 2.321196 \\
\hline 9 & 6 & 0 & 3.973969 & -1.766204 & 3.317084 \\
\hline 10 & 1 & 0 & 4.485089 & -1.121635 & 1.326579 \\
\hline 11 & 6 & 0 & 2.950278 & -1.994648 & 4.231760 \\
\hline 12 & 1 & 0 & 0.822592 & -1.916358 & 4.581714 \\
\hline 13 & 1 & 0 & 5.009301 & -1.957071 & 3.596686 \\
\hline 14 & 1 & 0 & 3.183424 & -2.362631 & 5.230031 \\
\hline 15 & 6 & 0 & -2.088868 & -1.532173 & 1.105002 \\
\hline 16 & 6 & 0 & -2.637394 & -1.429045 & 2.387656 \\
\hline 17 & 6 & 0 & -1.408859 & -2.708080 & 0.758755 \\
\hline 18 & 6 & 0 & -2.513922 & -2.476128 & 3.297686 \\
\hline 19 & 1 & 0 & -3.165271 & -0.520715 & 2.679663 \\
\hline 20 & 6 & 0 & -1.294151 & -3.754986 & 1.665609 \\
\hline 21 & 1 & 0 & -0.956035 & -2.805516 & -0.229276 \\
\hline 22 & 6 & 0 & -1.844255 & -3.642114 & 2.940001 \\
\hline 23 & 1 & 0 & -2.949483 & -2.378346 & 4.291412 \\
\hline 24 & 1 & 0 & -0.758931 & -4.657715 & 1.372964 \\
\hline 25 & 1 & 0 & -1.747933 & -4.459013 & 3.653610 \\
\hline 26 & 6 & 0 & 3.456542 & 0.128122 & -0.701312 \\
\hline 27 & 6 & 0 & 4.295921 & -0.660864 & -1.491468 \\
\hline 28 & 6 & 0 & 3.840191 & 1.441155 & -0.404202 \\
\hline 29 & 6 & 0 & 5.496720 & -0.146258 & -1.974217 \\
\hline 30 & 1 & 0 & 4.010502 & -1.685838 & -1.729293 \\
\hline 31 & 6 & 0 & 5.045782 & 1.948988 & -0.873905 \\
\hline 32 & 1 & 0 & 3.175301 & 2.063119 & 0.200935 \\
\hline 33 & 6 & 0 & 5.874588 & 1.156071 & -1.664369 \\
\hline 34 & 1 & 0 & 6.140886 & -0.769075 & -2.593686 \\
\hline 35 & 1 & 0 & 5.335784 & 2.970176 & -0.630381 \\
\hline 36 & 1 & 0 & 6.814276 & 1.555987 & -2.042757 \\
\hline 37 & 6 & 0 & 1.575118 & -2.030602 & -0.905603 \\
\hline 38 & 6 & 0 & 0.856192 & -1.951814 & -2.103737 \\
\hline 39 & 6 & 0 & 1.965132 & -3.286772 & -0.433600 \\
\hline 40 & 6 & 0 & 0.522275 & -3.100289 & -2.812442 \\
\hline 41 & 1 & 0 & 0.524501 & -0.976609 & -2.465286 \\
\hline 42 & 6 & 0 & 1.630477 & -4.438587 & -1.141989 \\
\hline 43 & 1 & 0 & 2.519771 & -3.366226 & 0.502136 \\
\hline 44 & 6 & 0 & 0.905472 & -4.348931 & -2.327179 \\
\hline 45 & 1 & 0 & -0.062658 & -3.018251 & -3.728473 \\
\hline 46 & 1 & 0 & 1.935071 & -5.413051 & -0.761609 \\
\hline 47 & 1 & 0 & 0.635195 & -5.252858 & -2.871186 \\
\hline 48 & 6 & 0 & -3.536189 & 0.851532 & 0.467450 \\
\hline 49 & 6 & 0 & -4.820005 & 0.320981 & 0.302563 \\
\hline 50 & 6 & 0 & -3.390630 & 2.121743 & 1.026811 \\
\hline 51 & 6 & 0 & -5.936897 & 1.050866 & 0.689174 \\
\hline 52 & 1 & 0 & -4.944875 & -0.669584 & -0.137299 \\
\hline 53 & 6 & 0 & -4.510236 & 2.851381 & 1.420998 \\
\hline 54 & 1 & 0 & -2.385057 & 2.534975 & 1.138662 \\
\hline 55 & 6 & 0 & -5.782311 & 2.317132 & 1.251118 \\
\hline 56 & 1 & 0 & -6.933078 & 0.631978 & 0.552841 \\
\hline 57 & 1 & 0 & -4.386129 & 3.841532 & 1.857366 \\
\hline 58 & 1 & 0 & -6.659145 & 2.888745 & 1.552420 \\
\hline 59 & 6 & 0 & -2.458864 & -0.741392 & -1.656779 \\
\hline 60 & 6 & 0 & -3.135565 & -1.944875 & -1.874314 \\
\hline 61 & 6 & 0 & -2.070279 & 0.021817 & -2.763640 \\
\hline 62 & 6 & 0 & -3.412627 & -2.375516 & -3.168713 \\
\hline 63 & 1 & 0 & -3.432609 & -2.562845 & -1.026366 \\
\hline 64 & 6 & 0 & -2.355200 & -0.402148 & -4.056766 \\
\hline 65 & 1 & 0 & -1.521689 & 0.952091 & -2.595680 \\
\hline 66 & 6 & 0 & -3.024728 & -1.605892 & -4.261588 \\
\hline 67 & 1 & 0 & -3.934505 & -3.318986 & -3.323371 \\
\hline 68 & 1 & 0 & -2.047905 & 0.204244 & -4.907623 \\
\hline
\end{tabular}




$\begin{array}{rrrrrr}69 & 1 & 0 & -3.243373 & -1.944422 & -5.273346 \\ 70 & 6 & 0 & 0.470851 & 4.233726 & 0.873604 \\ 71 & 1 & 0 & 0.310691 & 4.204564 & 1.949202 \\ 72 & 6 & 0 & 0.372537 & 3.175463 & 0.001159 \\ 73 & 6 & 0 & 0.648898 & 3.523170 & -1.327295 \\ 74 & 1 & 0 & 1.622425 & 3.283859 & -1.759704 \\ 75 & 6 & 0 & 0.813933 & 5.442335 & 0.191149 \\ 76 & 8 & 0 & 0.989629 & 5.416860 & -1.056097 \\ 77 & 6 & 0 & 0.966726 & 6.746756 & 0.905157 \\ 78 & 1 & 0 & 0.815568 & 6.644929 & 1.984997 \\ 79 & 1 & 0 & 0.245153 & 7.471214 & 0.507518 \\ 80 & 1 & 0 & -0.158709 & 3.644115 & -2.050582 \\ 81 & 1 & 0 & 1.966156 & 7.157760 & 0.717413 \\ ------------------------------------------------ & & \end{array}$

Zero-point correction= 0.644187 (Hartree/Particle)

Thermal correction to Energy= 0.644187
0.686698

Thermal correction to Gibbs Free Energy=

Sum of electronic and zero-point Energies

Sum of electronic and thermal Energies=
Sum of electronic and thermal Enthalpies=

0.561795

$-2466.679739$

$-2466.637228$

2466.63628

Sum of electronic and thermal Free Energies= $\quad-2466.762131$

M06 /6-311++G(d,p)-SDD/SMD//M06/6-31G(d)-LANL2DZ energy in toluene solvent = -2469.0142989

INT2,

\begin{tabular}{|c|c|c|c|c|c|}
\hline \multirow{2}{*}{$\begin{array}{l}\text { Center } \\
\text { Number }\end{array}$} & \multirow{2}{*}{$\begin{array}{l}\text { Atomic } \\
\text { Number }\end{array}$} & \multirow{2}{*}{$\begin{array}{l}\text { Atomic } \\
\text { Type }\end{array}$} & \multicolumn{3}{|c|}{ Coordinates (Angstroms) } \\
\hline & & & $\mathrm{X}$ & $\mathrm{Y}$ & \\
\hline 1 & 15 & 0 & 1.678291 & -0.712280 & -0.009200 \\
\hline 2 & 46 & 0 & 0.205241 & 1.169609 & -0.099857 \\
\hline 3 & 15 & 0 & -2.000730 & 0.236066 & -0.046911 \\
\hline 4 & 6 & 0 & 2.124740 & -1.253616 & 1.690500 \\
\hline 5 & 6 & 0 & 1.100649 & -1.298676 & 2.644515 \\
\hline 6 & 6 & 0 & 3.417309 & -1.630314 & 2.066577 \\
\hline 7 & 6 & 0 & 1.362487 & -1.726424 & 3.941280 \\
\hline 8 & 1 & 0 & 0.087196 & -1.000576 & 2.367541 \\
\hline 9 & 6 & 0 & 3.679419 & -2.046376 & 3.369009 \\
\hline 10 & 1 & 0 & 4.226602 & -1.598081 & 1.336988 \\
\hline 11 & 6 & 0 & 2.653184 & -2.097989 & 4.307147 \\
\hline 12 & 1 & 0 & 0.551900 & -1.761848 & 4.668023 \\
\hline 13 & 1 & 0 & 4.692079 & -2.332803 & 3.650177 \\
\hline 14 & 1 & 0 & 2.860711 & -2.423914 & 5.325512 \\
\hline 15 & 6 & 0 & -2.281059 & -1.109069 & 1.176173 \\
\hline 16 & 6 & 0 & -2.748503 & -0.814871 & 2.462244 \\
\hline 17 & 6 & 0 & -1.825211 & -2.407108 & 0.909681 \\
\hline 18 & 6 & 0 & -2.767881 & -1.794593 & 3.451083 \\
\hline 19 & 1 & 0 & -3.097405 & 0.191826 & 2.694425 \\
\hline 20 & 6 & 0 & -1.853248 & -3.385887 & 1.896057 \\
\hline 21 & 1 & 0 & -1.431704 & -2.652054 & -0.077858 \\
\hline 22 & 6 & 0 & -2.323731 & -3.083571 & 3.171235 \\
\hline 23 & 1 & 0 & -3.137341 & -1.547815 & 4.445724 \\
\hline 24 & 1 & 0 & -1.494279 & -4.388223 & 1.665317 \\
\hline 25 & 1 & 0 & -2.340302 & -3.849099 & 3.945533 \\
\hline 26 & 6 & 0 & 3.330677 & -0.421969 & -0.769756 \\
\hline 27 & 6 & 0 & 4.044041 & -1.399324 & -1.468637 \\
\hline 28 & 6 & 0 & 3.899500 & 0.846369 & -0.610175 \\
\hline 29 & 6 & 0 & 5.299200 & -1.112284 & -1.999140 \\
\hline 30 & 1 & 0 & 3.616898 & -2.394053 & -1.597274 \\
\hline 31 & 6 & 0 & 5.157700 & 1.128971 & -1.129579 \\
\hline 32 & 1 & 0 & 3.333542 & 1.612854 & -0.074294 \\
\hline 33 & 6 & 0 & 5.858440 & 0.150098 & -1.829980 \\
\hline 34 & 1 & 0 & 5.843027 & -1.882184 & -2.545023 \\
\hline 35 & 1 & 0 & 5.591206 & 2.119136 & -0.993861 \\
\hline 36 & 1 & 0 & 6.839774 & 0.372448 & -2.246717 \\
\hline 37 & 6 & 0 & 1.165581 & -2.291024 & -0.805342 \\
\hline 38 & 6 & 0 & 0.492233 & -2.191836 & -2.028301 \\
\hline 39 & 6 & 0 & 1.334633 & -3.552507 & -0.228788 \\
\hline 40 & 6 & 0 & -0.022946 & -3.320894 & -2.653805 \\
\hline 41 & 1 & 0 & 0.341491 & -1.207538 & -2.476112 \\
\hline 42 & 6 & 0 & 0.825148 & -4.686543 & -0.857928 \\
\hline 43 & 1 & 0 & 1.850920 & -3.647336 & 0.727153 \\
\hline 44 & 6 & 0 & 0.139319 & -4.573148 & -2.063896 \\
\hline 45 & 1 & 0 & -0.573596 & -3.213524 & -3.588492 \\
\hline 46 & 1 & 0 & 0.958102 & -5.664853 & -0.396836 \\
\hline 47 & 1 & 0 & -0.272606 & -5.460600 & -2.542284 \\
\hline 48 & 6 & 0 & -3.365466 & 1.403749 & 0.377623 \\
\hline 49 & 6 & 0 & -4.716759 & 1.050521 & 0.294561 \\
\hline 50 & 6 & 0 & -3.022431 & 2.677697 & 0.831677 \\
\hline 51 & 6 & 0 & -5.704718 & 1.956212 & 0.659891 \\
\hline 52 & 1 & 0 & -4.999012 & 0.057929 & -0.058390 \\
\hline 53 & 6 & 0 & -4.012518 & 3.584027 & 1.205344 \\
\hline 54 & 1 & 0 & -1.964233 & 2.948104 & 0.877835 \\
\hline 55 & 6 & 0 & -5.352539 & 3.224336 & 1.118775 \\
\hline 56 & 1 & 0 & -6.754132 & 1.673269 & 0.589274 \\
\hline 57 & 1 & 0 & -3.734054 & 4.575231 & 1.560738 \\
\hline 58 & 1 & 0 & -6.128043 & 3.933092 & 1.406344 \\
\hline 59 & 6 & 0 & -2.581299 & -0.490006 & -1.635925 \\
\hline 60 & 6 & 0 & -3.471310 & -1.562312 & -1.746671 \\
\hline 61 & 6 & 0 & -2.065333 & 0.079200 & -2.805428 \\
\hline 62 & 6 & 0 & -3.827169 & -2.059596 & -2.996632 \\
\hline 63 & 1 & 0 & -3.875203 & -2.029224 & -0.848134 \\
\hline 64 & 6 & 0 & -2.425273 & -0.412044 & -4.055753 \\
\hline
\end{tabular}




$\begin{array}{lllrrc}65 & 1 & 0 & -1.356460 & 0.906251 & -2.718393 \\ 66 & 6 & 0 & -3.304575 & -1.487471 & -4.152970 \\ 67 & 1 & 0 & -4.516463 & -2.900016 & -3.067046 \\ 68 & 1 & 0 & -2.013224 & 0.040744 & -4.956552 \\ 69 & 1 & 0 & -3.583841 & -1.879113 & -5.130122 \\ 70 & 6 & 0 & 1.323316 & 3.980906 & 0.795613 \\ 71 & 1 & 0 & 1.167996 & 3.872447 & 1.865581 \\ 72 & 6 & 0 & 0.938890 & 3.051327 & -0.205919 \\ 73 & 6 & 0 & 1.409325 & 3.711403 & -1.452547 \\ 74 & 1 & 0 & 2.180859 & 3.123512 & -1.970249 \\ 75 & 6 & 0 & 1.932084 & 5.070699 & 0.221202 \\ 76 & 8 & 0 & 2.021608 & 4.997094 & -1.102415 \\ 77 & 6 & 0 & 2.489453 & 6.292697 & 0.841366 \\ 78 & 1 & 0 & 2.371904 & 6.268817 & 1.928966 \\ 79 & 1 & 0 & 1.987760 & 7.185954 & 0.446687 \\ 80 & 1 & 0 & 0.604781 & 3.930752 & -2.165337 \\ 81 & 1 & 0 & 3.555633 & 6.392348 & 0.599271\end{array}$

Zero-point correction=

0.647319 (Hartree/Particle)

Thermal correction to Energy=

0.688485

Thermal correction to Enthalpy=

Thermal correction to Gibbs Free Energy=

Sum of electronic and zero-point Energies=

Sum of electronic and thermal Energies=

Sum of electronic and thermal Enthalpies=

0.570284

$-2466.694297$

$-2466.653131$

-2466.653131
-2466.652186

Sum of electronic and thermal Free Energies $=\quad-2466.771332$

M06 /6-311++G(d,p)-SDD/SMD//M06/6-31G(d)-LANL2DZ energy in toluene solvent $=-2469.0303695$

INT4'

\begin{tabular}{|c|c|c|c|c|c|}
\hline \multirow{2}{*}{$\begin{array}{l}\text { Center } \\
\text { Number }\end{array}$} & \multirow{2}{*}{$\begin{array}{l}\text { Atomic } \\
\text { Number }\end{array}$} & \multirow{2}{*}{$\begin{array}{l}\text { Atomic } \\
\text { Type }\end{array}$} & \multicolumn{3}{|c|}{ Coordinates (Angstroms) } \\
\hline & & & $\mathrm{X}$ & $\mathrm{Y}$ & Z \\
\hline 1 & 6 & 0 & -2.129008 & 4.604531 & 0.083776 \\
\hline 2 & 6 & 0 & -1.759766 & 3.966507 & -1.097401 \\
\hline 3 & 6 & 0 & -0.941884 & 2.838539 & -1.057741 \\
\hline 4 & 6 & 0 & -0.480597 & 2.342997 & 0.162917 \\
\hline 5 & 6 & 0 & -0.845978 & 2.988914 & 1.343845 \\
\hline 6 & 6 & 0 & -1.671569 & 4.113868 & 1.302832 \\
\hline 7 & 1 & 0 & -2.118591 & 4.339955 & -2.056401 \\
\hline 8 & 1 & 0 & -0.681003 & 2.335898 & -1.992453 \\
\hline 9 & 1 & 0 & -0.489775 & 2.617793 & 2.307182 \\
\hline 10 & 1 & 0 & -1.953623 & 4.609454 & 2.231871 \\
\hline 11 & 53 & 0 & 2.566010 & -1.387296 & 0.365281 \\
\hline 12 & 46 & 0 & 0.824454 & 0.766504 & 0.232000 \\
\hline 13 & 15 & 0 & -0.995968 & -0.676407 & -0.063560 \\
\hline 14 & 6 & 0 & 2.468782 & 2.401453 & -0.002611 \\
\hline 15 & 1 & 0 & 1.794970 & 3.174089 & -0.383367 \\
\hline 16 & 6 & 0 & 2.477514 & 2.113157 & 1.303767 \\
\hline 17 & 6 & 0 & 2.634892 & 1.924286 & 2.583347 \\
\hline 18 & 1 & 0 & 3.300835 & 2.575648 & 3.149421 \\
\hline 19 & 6 & 0 & 3.558813 & 1.930505 & -0.939585 \\
\hline 20 & 8 & 0 & 4.702541 & 1.824907 & -0.566067 \\
\hline 21 & 6 & 0 & -2.595145 & 0.063663 & -0.561736 \\
\hline 22 & 6 & 0 & -3.101400 & -0.050762 & -1.856833 \\
\hline 23 & 6 & 0 & -3.299487 & 0.819269 & 0.383579 \\
\hline 24 & 6 & 0 & -4.288685 & 0.590043 & -2.204124 \\
\hline 25 & 1 & 0 & -2.573554 & -0.644609 & -2.601601 \\
\hline 26 & 6 & 0 & -4.485361 & 1.449649 & 0.036624 \\
\hline 27 & 1 & 0 & -2.905672 & 0.928144 & 1.394525 \\
\hline 28 & 6 & 0 & -4.980336 & 1.340461 & -1.261665 \\
\hline 29 & 1 & 0 & -4.675170 & 0.493536 & -3.217452 \\
\hline 30 & 1 & 0 & -5.018075 & 2.041078 & 0.779318 \\
\hline 31 & 1 & 0 & -5.906809 & 1.842364 & -1.535783 \\
\hline 32 & 6 & 0 & -0.625232 & -1.884469 & -1.377800 \\
\hline 33 & 6 & 0 & -0.806537 & -3.256472 & -1.211736 \\
\hline 34 & 6 & 0 & -0.102522 & -1.398230 & -2.581011 \\
\hline 35 & 6 & 0 & -0.474519 & -4.130903 & -2.242338 \\
\hline 36 & 1 & 0 & -1.182853 & -3.648037 & -0.267787 \\
\hline 37 & 6 & 0 & 0.214026 & -2.271430 & -3.611922 \\
\hline 38 & 1 & 0 & 0.074646 & -0.326772 & -2.699832 \\
\hline 39 & 6 & 0 & 0.030468 & -3.641990 & -3.441287 \\
\hline 40 & 1 & 0 & -0.604711 & -5.202407 & -2.101399 \\
\hline 41 & 1 & 0 & 0.620565 & -1.884901 & -4.544861 \\
\hline 42 & 1 & 0 & 0.292874 & -4.329541 & -4.243387 \\
\hline 43 & 6 & 0 & -1.447086 & -1.616746 & 1.434631 \\
\hline 44 & 6 & 0 & -0.597032 & -1.640501 & 2.541524 \\
\hline 45 & 6 & 0 & -2.659540 & -2.317543 & 1.481572 \\
\hline 46 & 6 & 0 & -0.949860 & -2.361688 & 3.678323 \\
\hline 47 & 1 & 0 & 0.353994 & -1.108964 & 2.498574 \\
\hline 48 & 6 & 0 & -3.003796 & -3.042843 & 2.614840 \\
\hline 49 & 1 & 0 & -3.336611 & -2.290650 & 0.626884 \\
\hline 50 & 6 & 0 & -2.149123 & -3.063711 & 3.715044 \\
\hline 51 & 1 & 0 & -0.279662 & -2.376988 & 4.535662 \\
\hline 52 & 1 & 0 & -3.944465 & -3.590193 & 2.641556 \\
\hline 53 & 1 & 0 & -2.422316 & -3.630049 & 4.603947 \\
\hline 54 & 6 & 0 & 3.118826 & 1.637357 & -2.347440 \\
\hline 55 & 1 & 0 & 2.235659 & 2.222529 & -2.637005 \\
\hline 56 & 1 & 0 & 2.846365 & 0.572274 & -2.393093 \\
\hline 57 & 1 & 0 & -2.771126 & 5.483602 & 0.054098 \\
\hline 58 & 1 & 0 & 2.141745 & 1.114223 & 3.117694 \\
\hline 59 & 1 & 0 & 3.944977 & 1.809421 & -3.044575 \\
\hline
\end{tabular}


Zero-point correction=

Thermal correction to Energy=

Thermal correction to Enthalpy=

Thermal correction to Gibbs Free Energy=

Sum of electronic and zero-point Energies=

Sum of electronic and thermal Energies=

Sum of electronic and thermal Enthalpies=

Sum of electronic and thermal Free Energies=

TS2

\begin{tabular}{|c|c|c|c|c|c|}
\hline \multirow{2}{*}{$\begin{array}{l}\text { Center } \\
\text { Number }\end{array}$} & \multirow{2}{*}{$\begin{array}{l}\text { Atomic } \\
\text { Number }\end{array}$} & \multirow{2}{*}{$\begin{array}{l}\text { Atomic } \\
\text { Type }\end{array}$} & \multicolumn{3}{|c|}{ Coordinates (Angstroms) } \\
\hline & & & $\mathrm{X}$ & $\mathrm{Y}$ & Z \\
\hline 1 & 6 & 0 & -1.703427 & -4.411567 & 1.351713 \\
\hline 2 & 6 & 0 & -0.500704 & -4.015729 & 1.926159 \\
\hline 3 & 6 & 0 & 0.301480 & -3.066317 & 1.292361 \\
\hline 4 & 6 & 0 & -0.114447 & -2.498783 & 0.086094 \\
\hline 5 & 6 & 0 & -1.305462 & -2.910732 & -0.501598 \\
\hline 6 & 6 & 0 & -2.100147 & -3.859406 & 0.135904 \\
\hline 7 & 1 & 0 & -0.175606 & -4.443198 & 2.873778 \\
\hline 8 & 1 & 0 & 1.234919 & -2.752402 & 1.761581 \\
\hline 9 & 1 & 0 & -1.635617 & -2.493424 & -1.451987 \\
\hline 10 & 1 & 0 & -3.039234 & -4.162627 & -0.326251 \\
\hline 11 & 53 & 0 & 2.369936 & 1.232298 & 1.178014 \\
\hline 12 & 46 & 0 & 0.945568 & -0.700943 & -0.142979 \\
\hline 13 & 15 & 0 & -0.984521 & 0.684358 & -0.094533 \\
\hline 14 & 6 & 0 & 2.674900 & -1.956194 & -0.658582 \\
\hline 15 & 1 & 0 & 2.967118 & -2.536949 & 0.219845 \\
\hline 16 & 6 & 0 & 1.554898 & -2.325419 & -1.400207 \\
\hline 17 & 6 & 0 & 1.104798 & -2.722163 & -2.574176 \\
\hline 18 & 1 & 0 & 1.792654 & -3.188825 & -3.279520 \\
\hline 19 & 6 & 0 & 3.719464 & -1.094138 & -1.296082 \\
\hline 20 & 8 & 0 & 3.537737 & -0.530034 & -2.357360 \\
\hline 21 & 6 & 0 & -2.455497 & 0.009292 & -0.957348 \\
\hline 22 & 6 & 0 & -3.628913 & -0.380750 & -0.314179 \\
\hline 23 & 6 & 0 & -2.335622 & -0.183021 & -2.340156 \\
\hline 24 & 6 & 0 & -4.663009 & -0.968526 & -1.039922 \\
\hline 25 & 1 & 0 & -3.737872 & -0.235248 & 0.759903 \\
\hline 26 & 6 & 0 & -3.368917 & -0.765190 & -3.061924 \\
\hline 27 & 1 & 0 & -1.418228 & 0.117345 & -2.851518 \\
\hline 28 & 6 & 0 & -4.534186 & -1.166913 & -2.409785 \\
\hline 29 & 1 & 0 & -5.574423 & -1.271757 & -0.527035 \\
\hline 30 & 1 & 0 & -3.265488 & -0.908810 & -4.136247 \\
\hline 31 & 1 & 0 & -5.342645 & -1.630144 & -2.972884 \\
\hline 32 & 6 & 0 & -1.520760 & 1.051154 & 1.606344 \\
\hline 33 & 6 & 0 & -1.557088 & 2.346993 & 2.122696 \\
\hline 34 & 6 & 0 & -1.800798 & -0.034395 & 2.447043 \\
\hline 35 & 6 & 0 & -1.901404 & 2.557174 & 3.454357 \\
\hline 36 & 1 & 0 & -1.296489 & 3.194318 & 1.490471 \\
\hline 37 & 6 & 0 & -2.154692 & 0.182671 & 3.772855 \\
\hline 38 & 1 & 0 & -1.734042 & -1.055203 & 2.064290 \\
\hline 39 & 6 & 0 & -2.207087 & 1.479605 & 4.277450 \\
\hline 40 & 1 & 0 & -1.921111 & 3.570739 & 3.850827 \\
\hline 41 & 1 & 0 & -2.377042 & -0.665997 & 4.417304 \\
\hline 42 & 1 & 0 & -2.473378 & 1.648372 & 5.319533 \\
\hline 43 & 6 & 0 & -0.786049 & 2.264118 & -0.991545 \\
\hline 44 & 6 & 0 & 0.389456 & 2.536170 & -1.693421 \\
\hline 45 & 6 & 0 & -1.858370 & 3.162753 & -1.066824 \\
\hline 46 & 6 & 0 & 0.499333 & 3.705185 & -2.441809 \\
\hline 47 & 1 & 0 & 1.224614 & 1.836271 & -1.650665 \\
\hline 48 & 6 & 0 & -1.738784 & 4.334658 & -1.802133 \\
\hline 49 & 1 & 0 & -2.793546 & 2.940856 & -0.550189 \\
\hline 50 & 6 & 0 & -0.557402 & 4.606471 & -2.489380 \\
\hline 51 & 1 & 0 & 1.420230 & 3.909063 & -2.984548 \\
\hline 52 & 1 & 0 & -2.572494 & 5.033314 & -1.847293 \\
\hline 53 & 1 & 0 & -0.466352 & 5.523091 & -3.070000 \\
\hline 54 & 6 & 0 & 5.016892 & -1.007693 & -0.539898 \\
\hline 55 & 1 & 0 & 4.837632 & -0.527990 & 0.433320 \\
\hline 56 & 1 & 0 & 5.736681 & -0.415527 & -1.111645 \\
\hline 57 & 1 & 0 & 5.426101 & -2.008839 & -0.346427 \\
\hline 58 & 1 & 0 & 0.063183 & -2.630502 & -2.874208 \\
\hline 59 & 1 & 0 & -2.327129 & -5.155285 & 1.844727 \\
\hline Zero-p & orrection= & & & 0.460640 & Fartree/Particle \\
\hline Therma & correction to & nergy $=$ & & 0.492982 & \\
\hline Therma & orrection to & nthalpy= & & 0.493926 & \\
\hline Therma & orrection to & ibbs Free E & & 0.394548 & \\
\hline Sum of & lectronic and & ro-point $\mathrm{El}$ & & -1673.9837 & \\
\hline Sum of & ctronic and & ermal Ener & & -1673.951 & \\
\hline Sum of & ctronic and & ermal Enth & & -1673.9504 & \\
\hline um o & onic ar & 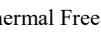 & gles= & 1674.0498 & \\
\hline
\end{tabular}

$\begin{array}{ll}1.2 .525419 & -1.400207\end{array}$

$\begin{array}{lll}1.7926464 & -1.094138 & -1.29608 \\ 3.537737 & -0.530334 & -2.357360\end{array}$

$\begin{array}{rrr}3.537737 & -0.530034 & -2.357360 \\ -2.455497 & 0.009292 & -0.957348\end{array}$

$\begin{array}{ll}-0.380750 & -0.314179\end{array}$

$\begin{array}{lll}-2.335622 & -0.183021 & -2.340156\end{array}$

$\begin{array}{lll}-4.663009 & -0.968526 & -1.039922\end{array}$

$\begin{array}{lll}-3.737872 & -0.235248 & 0.759903\end{array}$

$\begin{array}{rrr}-3.368917 & -0.765190 & -3.061924 \\ -1.418228 & 0.117345 & -2.851518\end{array}$

$\begin{array}{lll}-4.534186 & -1.166913 & -2.409785\end{array}$

$\begin{array}{lll}-5.574423 & -1.271757 & -0.527035\end{array}$

$\begin{array}{lll}-0.908810 & -4.136247\end{array}$

$\begin{array}{lll}1.557088 & 2.346993 & 2.122696\end{array}$

2.447043

1.49047

$\begin{array}{lll}1.296489 & 3.194318 & 1.490471 \\ -1.73692 & 0.182671 & 3.772855\end{array}$

$\begin{array}{lll}-1.734042 & -1.055203 & 2.064290\end{array}$

$\begin{array}{lll}-1.921111 & 3.570739 & 3.850827\end{array}$

$\begin{array}{lll}-2.377042 & -0.665997 & 4.417304\end{array}$

$\begin{array}{llr}-0.786049 & 2.264118 & -0.991545 \\ 0.389456 & 2.536170 & -1.693421\end{array}$

$\begin{array}{lll}0.389456 & 2.536170 & -1.693421\end{array}$

$\begin{array}{lll}-1.858370 & 3.162753 & -1.066824\end{array}$

$\begin{array}{ll}3.705185 & -2.441809 \\ 1.836271 & -1.650655\end{array}$

$-1.650665$

$\begin{array}{lll}-1.738784 & 4.334658 & -1.802133 \\ -2.793546 & 2.940856 & -0.550189\end{array}$

$\begin{array}{ll}4.606471 & -2.489380 \\ 3.909063 & -2.984548\end{array}$

$\begin{array}{llll}-0.466352 & 5.523091 & -3.070000\end{array}$

\begin{tabular}{lll}
66352 & -523091 & -3.07000 \\
\hline 37632 & -0.52793 & -0.539898
\end{tabular}

$\begin{array}{rrr}4.837632 & -0.527990 & 0.433320 \\ 5.736681 & -0.415527 & -1.111645 \\ 5.426101 & -2.008839 & -0.346427\end{array}$

$\begin{array}{lll}5.426101 & -2.008839 & -0.346427\end{array}$

$\begin{array}{lll}0.063183 & -2.630502 & -2.874208\end{array}$

0.460640 (Hartree/Particle)

0.492982

0.394548

$-1673.951407$

$-1674.049841$
0.460794 (Hartree/Particle)

0.49421

0.49515

2068

$-1673.998888$

$-1673.965471$

DZZ energy in toluene solvent $=-1676.0366884$

M06 /6-311++G(d,p)-SDD/SMD//M06/6-31G(d)-LANL2DZ energy in toluene solvent = -1676.0211844

TS2"

\begin{tabular}{cccccc}
$\begin{array}{c}\text { Center } \\
\text { Number }\end{array}$ & $\begin{array}{c}\text { Atomic } \\
\text { Number }\end{array}$ & $\begin{array}{c}\text { Atomic } \\
\text { Type }\end{array}$ & \multicolumn{2}{c}{ Coordinates (Angstroms) } & X \\
- \\
-1 & 6 & 0 & 1.046481 & 4.749165 & 0.299765 \\
2 & 6 & 0 & 0.505482 & 4.159760 & 1.439871 \\
3 & 6 & 0 & -0.250857 & 2.995124 & 1.335871
\end{tabular}




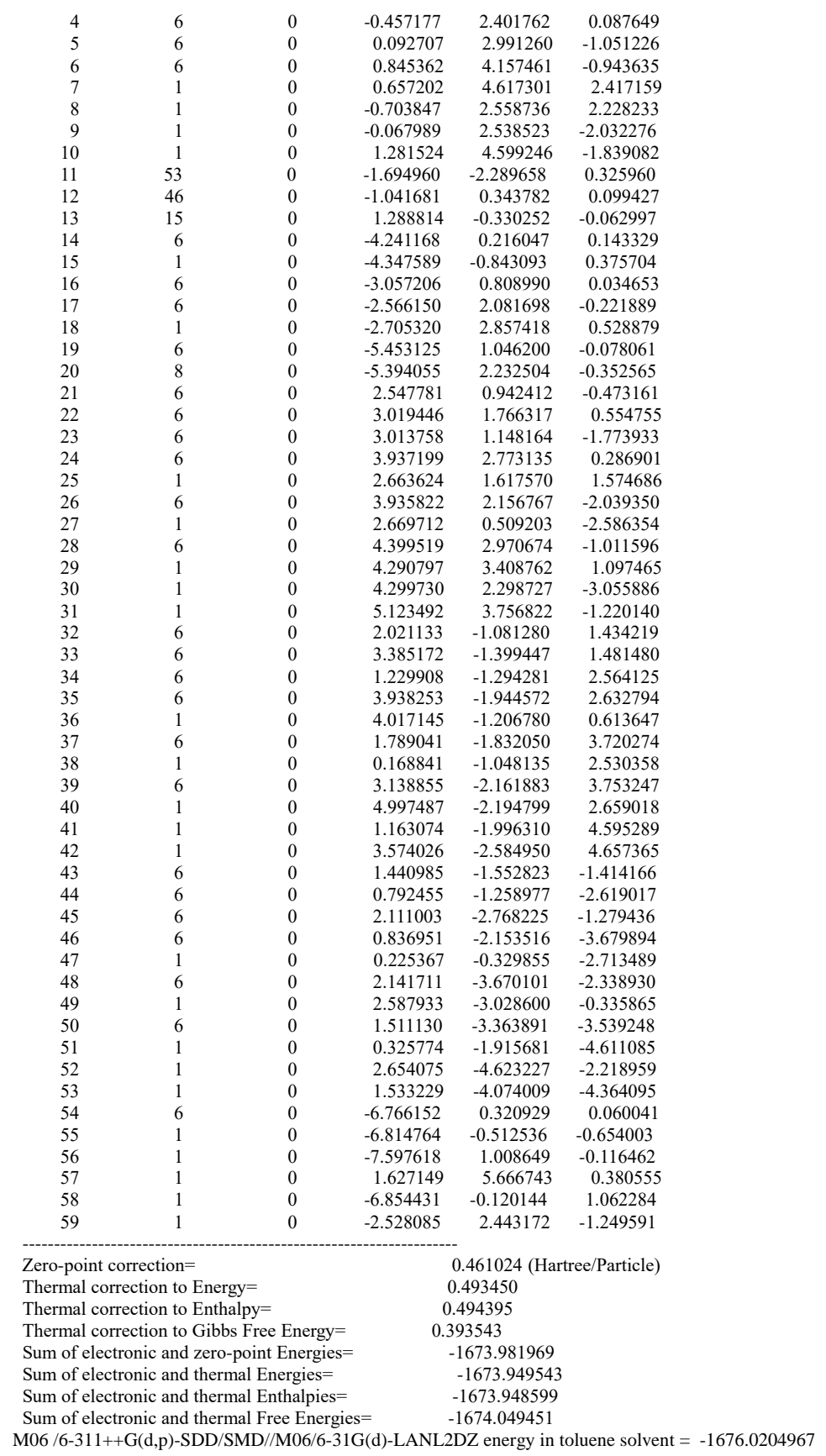

TS2"'

\begin{tabular}{rrrrrr} 
Center & Atomic & Atomic & \multicolumn{2}{c}{ Coordinates (Angstroms) } \\
Number & Number & Type & X & Y & $Z$ \\
--1 & 6 & 0 & 3.281388 & 3.006694 & -1.435074 \\
2 & 6 & 0 & 3.096404 & 2.048278 & -2.431177 \\
3 & 6 & 0 & 2.588243 & 0.794284 & -2.108218 \\
4 & 6 & 0 & 2.243608 & 0.494111 & -0.784721 \\
5 & 6 & 0 & 2.475839 & 1.434556 & 0.218108 \\
6 & 6 & 0 & 2.972238 & 2.694727 & -0.114992 \\
7 & 1 & 0 & 3.349322 & 2.277195 & -3.466053 \\
8 & 1 & 0 & 2.451183 & 0.041989 & -2.887517 \\
9 & 1 & 0 & 2.255064 & 1.197221 & 1.259116 \\
10 & 1 & 0 & 3.116291 & 3.434064 & 0.672758 \\
11 & 53 & 0 & -1.234330 & -2.881202 & -0.661076 \\
12 & 46 & 0 & 0.729279 & -1.019368 & -0.547956 \\
13 & 15 & 0 & -0.933520 & 0.712209 & 0.115198 \\
14 & 6 & 0 & 2.975537 & -1.450613 & -0.381332 \\
15 & 1 & 0 & 3.670553 & -1.236808 & -1.195460 \\
16 & 6 & 0 & 2.131200 & -2.558563 & -0.583978 \\
17 & 6 & 0 & 2.137843 & -3.870297 & -0.711737 \\
18 & 1 & 0 & 1.236206 & -4.444670 & -0.910317 \\
19 & 6 & 0 & 3.521692 & -1.188561 & 0.998869 \\
20 & 8 & 0 & 2.855717 & -1.328355 & 2.000139 \\
21 & 6 & 0 & -0.357603 & 1.138262 & 1.800697
\end{tabular}




\begin{tabular}{|c|c|c|c|c|c|}
\hline 22 & 6 & 0 & -0.108124 & 2.445074 & 2.225982 \\
\hline 23 & 6 & 0 & -0.035525 & 0.068926 & 2.646401 \\
\hline 24 & 6 & 0 & 0.450755 & 2.677985 & 3.479745 \\
\hline 25 & 1 & 0 & -0.325289 & 3.285541 & 1.567680 \\
\hline 26 & 6 & 0 & 0.522931 & 0.305105 & 3.896530 \\
\hline 27 & 1 & 0 & -0.195233 & -0.957182 & 2.307122 \\
\hline 28 & 6 & 0 & 0.768585 & 1.610864 & 4.314015 \\
\hline 29 & 1 & 0 & 0.647128 & 3.700035 & 3.800598 \\
\hline 30 & 1 & 0 & 0.789606 & -0.536980 & 4.532012 \\
\hline 31 & 1 & 0 & 1.217637 & 1.795085 & 5.288784 \\
\hline 32 & 6 & 0 & -2.687032 & 0.234286 & 0.329837 \\
\hline 33 & 6 & 0 & -3.284951 & 0.064850 & 1.577325 \\
\hline 34 & 6 & 0 & -3.435302 & 0.004983 & -0.829549 \\
\hline 35 & 6 & 0 & -4.616877 & -0.333961 & 1.663788 \\
\hline 36 & 1 & 0 & -2.713617 & 0.240632 & 2.487916 \\
\hline 37 & 6 & 0 & -4.763143 & -0.384849 & -0.740051 \\
\hline 38 & 1 & 0 & -2.968107 & 0.117746 & -1.809053 \\
\hline 39 & 6 & 0 & -5.355406 & -0.560181 & 0.508841 \\
\hline 40 & 1 & 0 & -5.075699 & -0.468185 & 2.642153 \\
\hline 41 & 1 & 0 & -5.335091 & -0.568652 & -1.647810 \\
\hline 42 & 1 & 0 & -6.394666 & -0.877254 & 0.578836 \\
\hline 43 & 6 & 0 & -1.128995 & 2.318624 & -0.745084 \\
\hline 44 & 6 & 0 & -2.104662 & 3.237853 & -0.334871 \\
\hline 45 & 6 & 0 & -0.312954 & 2.633091 & -1.829728 \\
\hline 46 & 6 & 0 & -2.234423 & 4.457789 & -0.985633 \\
\hline 47 & 1 & 0 & -2.764644 & 2.990764 & 0.497780 \\
\hline 48 & 6 & 0 & -0.443948 & 3.855968 & -2.482211 \\
\hline 49 & 1 & 0 & 0.435861 & 1.917908 & -2.163514 \\
\hline 50 & 6 & 0 & -1.400970 & 4.769921 & -2.058901 \\
\hline 51 & 1 & 0 & -2.992211 & 5.167553 & -0.657638 \\
\hline 52 & 1 & 0 & 0.208413 & 4.088966 & -3.323025 \\
\hline 53 & 1 & 0 & -1.506388 & 5.727291 & -2.567065 \\
\hline 54 & 6 & 0 & 4.966868 & -0.768811 & 1.021749 \\
\hline 55 & 1 & 0 & 5.602627 & -1.575666 & 0.631360 \\
\hline 56 & 1 & 0 & 5.269644 & -0.531019 & 2.045166 \\
\hline 57 & 1 & 0 & 3.678854 & 3.988315 & -1.687021 \\
\hline 58 & 1 & 0 & 5.118419 & 0.104517 & 0.371711 \\
\hline 59 & 1 & 0 & 3.074582 & -4.422398 & -0.602377 \\
\hline Zero-p & & & \multicolumn{3}{|c|}{0.461916 (Hartree/Particle) } \\
\hline $\mathrm{P}$ & to & & \multicolumn{3}{|c|}{0.494012} \\
\hline Thermal & to & & \multicolumn{3}{|c|}{0.494956} \\
\hline Thermal & to & En & \multicolumn{3}{|c|}{0.396252} \\
\hline Sum of e & and & & \multicolumn{3}{|c|}{-1673.970150} \\
\hline Sum of e & and & erg & \multicolumn{3}{|c|}{-1673.938054} \\
\hline Sum of $\mathrm{e}$ & and & tha & \multicolumn{3}{|c|}{-1673.937109} \\
\hline Sum of $\mathrm{e}$ & & & \multicolumn{3}{|c|}{$\begin{array}{l}-1673.937109 \\
-1674.035814\end{array}$} \\
\hline
\end{tabular}

M06 /6-311++G(d,p)-SDD/SMD//M06/6-31G(d)-LANL2DZ energy in toluene solvent $=-1676.0071222$

TS7'

\begin{tabular}{rrrrrr} 
Center & Atomic & Atomic & \multicolumn{2}{c}{ Coordinates (Angstroms) } \\
Number & Number & Type & X & Y & \\
--------------1.0 & & \\
1 & 6 & 0 & -1.673883 & 4.592355 & 0.147398 \\
2 & 6 & 0 & -1.890463 & 3.836013 & 1.295815 \\
3 & 6 & 0 & -1.669982 & 2.458416 & 1.284156 \\
4 & 6 & 0 & -1.217847 & 1.820610 & 0.126466 \\
5 & 6 & 0 & -1.013676 & 2.585288 & -1.025025 \\
6 & 6 & 0 & -1.234562 & 3.961578 & -1.013603 \\
7 & 1 & 0 & -2.238764 & 4.318459 & 2.209644 \\
8 & 1 & 0 & -1.855863 & 1.878603 & 2.190048 \\
9 & 1 & 0 & -0.649610 & 2.113053 & -1.940510 \\
10 & 1 & 0 & -1.050968 & 4.543602 & -1.917055 \\
11 & 53 & 0 & -0.815107 & -2.996528 & -0.079489 \\
12 & 46 & 0 & -0.940069 & -0.197892 & 0.078764 \\
13 & 15 & 0 & 1.415426 & 0.094877 & 0.050657 \\
14 & 6 & 0 & -4.009927 & 0.566187 & -0.583414 \\
15 & 1 & 0 & -3.772807 & 1.398888 & -1.236134 \\
16 & 6 & 0 & -3.026190 & -0.273412 & 0.077436 \\
17 & 6 & 0 & -3.840161 & -1.169798 & 0.795898 \\
18 & 1 & 0 & -3.148062 & -1.502007 & -0.324023 \\
19 & 6 & 0 & -5.243678 & 0.174706 & -0.193222 \\
20 & 8 & 0 & -5.157238 & -0.909144 & 0.646154 \\
21 & 6 & 0 & 2.320525 & -0.418954 & 1.556761 \\
22 & 6 & 0 & 1.762693 & -1.385482 & 2.397741 \\
23 & 6 & 0 & 3.572735 & 0.123372 & 1.873211 \\
24 & 6 & 0 & 2.450923 & -1.808811 & 3.530585 \\
25 & 1 & 0 & 0.790740 & -1.815057 & 2.153546 \\
26 & 6 & 0 & 4.256885 & -0.302849 & 3.005361 \\
27 & 1 & 0 & 4.009990 & 0.889946 & 1.232926 \\
28 & 6 & 0 & 3.696088 & -1.269972 & 3.835527 \\
29 & 1 & 0 & 2.007874 & -2.563805 & 4.177639 \\
30 & 1 & 0 & 5.229741 & 0.125277 & 3.241892 \\
31 & 1 & 0 & 4.231539 & -1.600480 & 4.724322 \\
32 & 6 & 0 & 2.205377 & -0.790792 & -1.339563 \\
33 & 6 & 0 & 1.467888 & -0.936896 & -2.517694 \\
34 & 6 & 0 & 3.527231 & -1.236939 & -1.296541 \\
35 & 6 & 0 & 2.050114 & -1.504772 & -3.644284 \\
36 & 1 & 0 & 0.424874 & -0.614827 & -2.537787 \\
37 & 6 & 0 & 4.104852 & -1.815975 & -2.421809 \\
38 & 1 & 0 & 4.106378 & -1.140933 & -0.378407 \\
39 & 6 & 0 & 3.369885 & -1.945086 & -3.596127
\end{tabular}




\begin{tabular}{|c|c|c|c|c|c|}
\hline 40 & 1 & 0 & 1.468346 & -1.616228 & -4.557524 \\
\hline 41 & 1 & 0 & 5.133770 & -2.169596 & -2.379652 \\
\hline 42 & 1 & 0 & 3.824555 & -2.398803 & -4.475484 \\
\hline 43 & 6 & 0 & 1.984749 & 1.819505 & -0.197719 \\
\hline 44 & 6 & 0 & 1.750965 & 2.748242 & 0.823390 \\
\hline 45 & 6 & 0 & 2.526700 & 2.258903 & -1.406043 \\
\hline 46 & 6 & 0 & 2.046663 & 4.090211 & 0.635506 \\
\hline 47 & 1 & 0 & 1.313748 & 2.417121 & 1.766235 \\
\hline 48 & 6 & 0 & 2.820916 & 3.608046 & -1.593380 \\
\hline 49 & 1 & 0 & 2.716311 & 1.548752 & -2.210776 \\
\hline 50 & 6 & 0 & 2.578454 & 4.524246 & -0.577382 \\
\hline 51 & 1 & 0 & 1.849314 & 4.803714 & 1.433940 \\
\hline 52 & 1 & 0 & 3.241880 & 3.940979 & -2.540992 \\
\hline 53 & 1 & 0 & 2.802365 & 5.579209 & -0.728416 \\
\hline 54 & 6 & 0 & -6.611918 & 0.636994 & -0.501290 \\
\hline 55 & 1 & 0 & -6.574446 & 1.517498 & -1.149563 \\
\hline 56 & 1 & 0 & -7.152881 & 0.899717 & 0.416463 \\
\hline 57 & 1 & 0 & -3.572712 & -1.974289 & 1.473768 \\
\hline 58 & 1 & 0 & -7.187196 & -0.147333 & -1.009581 \\
\hline 59 & 1 & 0 & -1.845888 & 5.667923 & 0.157244 \\
\hline
\end{tabular}

0.491539

Thermal correction to Energy=

Thermal correction to Enthalpy=

Thermal correction to Gibbs Free Energy=

Sum of electronic and zero-point Energie

0.492483

Sum of electronic and thermal Energies=

0.391894

$-1673.975155$

Sum of electronic and thermal Enthalpies=

$-1673.942235$

Sum of electronic and thermal Free Energies $=\quad-1674.042824$

M06 /6-311++G(d,p)-SDD/SMD//M06/6-31G(d)-LANL2DZ energy in toluene solvent $=-1676.0100318$

TS7"

\begin{tabular}{|c|c|c|c|c|c|}
\hline \multirow{2}{*}{$\begin{array}{l}\text { Center } \\
\text { Number }\end{array}$} & \multirow{2}{*}{$\begin{array}{l}\text { Atomic } \\
\text { Number }\end{array}$} & \multirow{2}{*}{$\begin{array}{l}\text { Atomic } \\
\text { Type }\end{array}$} & \multicolumn{3}{|c|}{ Coordinates (Angstroms) } \\
\hline & & & $\mathrm{X}$ & $\mathrm{Y}$ & 2 \\
\hline 1 & 6 & 0 & -0.215124 & 5.076869 & -0.042738 \\
\hline 2 & 6 & 0 & -0.338695 & 4.545254 & -1.324036 \\
\hline 3 & 6 & 0 & -0.186369 & 3.175595 & -1.536025 \\
\hline 4 & 6 & 0 & 0.080741 & 2.305792 & -0.472026 \\
\hline 5 & 6 & 0 & 0.215833 & 2.855902 & 0.804729 \\
\hline 6 & 6 & 0 & 0.066225 & 4.226236 & 1.021132 \\
\hline 7 & 1 & 0 & -0.554591 & 5.201121 & -2.168293 \\
\hline 8 & 1 & 0 & -0.278207 & 2.777864 & -2.549152 \\
\hline 9 & 1 & 0 & 0.431268 & 2.209826 & 1.660321 \\
\hline 10 & 1 & 0 & 0.169153 & 4.629419 & 2.029131 \\
\hline 11 & 53 & 0 & 0.402593 & -2.433623 & -1.495365 \\
\hline 12 & 46 & 0 & 0.220968 & 0.307075 & -0.850549 \\
\hline 13 & 15 & 0 & -1.933121 & -0.085444 & 0.136647 \\
\hline 14 & 6 & 0 & 2.867526 & 1.891029 & -1.691162 \\
\hline 15 & 1 & 0 & 2.438514 & 2.884412 & -1.780124 \\
\hline 16 & 6 & 0 & 2.179124 & 0.694206 & -1.375872 \\
\hline 17 & 6 & 0 & 3.197321 & -0.286753 & -1.294362 \\
\hline 18 & 1 & 0 & 3.279262 & -0.542518 & 0.046948 \\
\hline 19 & 6 & 0 & 4.194205 & 1.585080 & -1.886065 \\
\hline 20 & 8 & 0 & 4.437697 & 0.290838 & -1.692073 \\
\hline 21 & 6 & 0 & -3.221326 & -0.983749 & -0.804977 \\
\hline 22 & 6 & 0 & -3.050537 & -1.182854 & -2.175223 \\
\hline 23 & 6 & 0 & -4.408944 & -1.397320 & -0.191035 \\
\hline 24 & 6 & 0 & -4.051053 & -1.798499 & -2.922377 \\
\hline 25 & 1 & 0 & -2.119560 & -0.873004 & -2.649381 \\
\hline 26 & 6 & 0 & -5.401254 & -2.020928 & -0.936398 \\
\hline 27 & 1 & 0 & -4.556784 & -1.230834 & 0.877123 \\
\hline 28 & 6 & 0 & -5.221704 & -2.221370 & -2.303889 \\
\hline 29 & 1 & 0 & -3.906616 & -1.956173 & -3.989700 \\
\hline 30 & 1 & 0 & -6.319833 & -2.347674 & -0.451369 \\
\hline 31 & 1 & 0 & -6.001105 & -2.709494 & -2.887250 \\
\hline 32 & 6 & 0 & -1.607269 & -1.094493 & 1.629278 \\
\hline 33 & 6 & 0 & -0.991377 & -0.497931 & 2.737162 \\
\hline 34 & 6 & 0 & -1.798256 & -2.478553 & 1.627681 \\
\hline 35 & 6 & 0 & -0.604657 & -1.265887 & 3.830665 \\
\hline 36 & 1 & 0 & -0.822377 & 0.580947 & 2.751748 \\
\hline 37 & 6 & 0 & -1.392052 & -3.247075 & 2.714650 \\
\hline 38 & 1 & 0 & -2.248577 & -2.961709 & 0.761841 \\
\hline 39 & 6 & 0 & -0.799174 & -2.644721 & 3.819126 \\
\hline 40 & 1 & 0 & -0.154736 & -0.782453 & 4.697610 \\
\hline 41 & 1 & 0 & -1.542204 & -4.325115 & 2.694338 \\
\hline 42 & 1 & 0 & -0.493788 & -3.247011 & 4.673517 \\
\hline 43 & 6 & 0 & -2.912475 & 1.346970 & 0.741281 \\
\hline 44 & 6 & 0 & -3.149291 & 2.370141 & -0.182444 \\
\hline 45 & 6 & 0 & -3.454473 & 1.453547 & 2.023402 \\
\hline 46 & 6 & 0 & -3.890369 & 3.488048 & 0.174832 \\
\hline 47 & 1 & 0 & -2.730698 & 2.296736 & -1.186664 \\
\hline 48 & 6 & 0 & -4.197802 & 2.575328 & 2.381040 \\
\hline 49 & 1 & 0 & -3.297308 & 0.660070 & 2.753620 \\
\hline 50 & 6 & 0 & -4.411753 & 3.595865 & 1.461573 \\
\hline 51 & 1 & 0 & -4.052145 & 4.281826 & -0.552759 \\
\hline 52 & 1 & 0 & -4.612456 & 2.649220 & 3.385514 \\
\hline 53 & 1 & 0 & -4.987296 & 4.475551 & 1.745842 \\
\hline 54 & 6 & 0 & 5.353795 & 2.436021 & -2.239551 \\
\hline 55 & 1 & 0 & 5.039411 & 3.474726 & -2.380425 \\
\hline 56 & 1 & 0 & 5.825615 & 2.082266 & -3.165336 \\
\hline 57 & 1 & 0 & 3.080519 & -1.298951 & -1.687773 \\
\hline
\end{tabular}




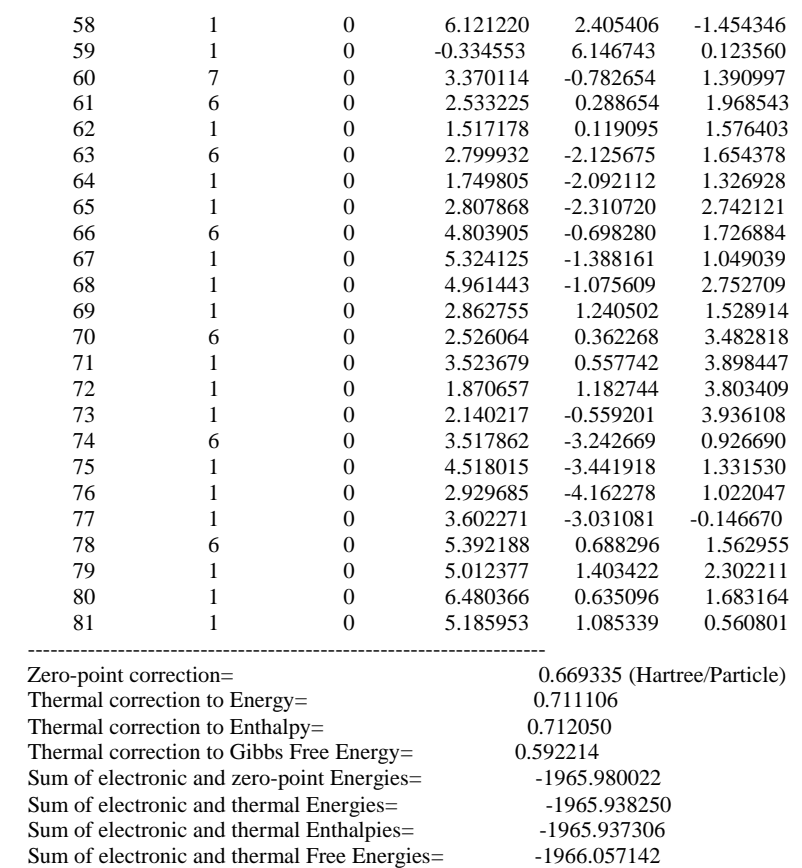

M06 /6-311++G(d,p)-SDD/SMD//M06/6-31G(d)-LANL2DZ energy in toluene solvent $=-1968.3156546$

TS8

\begin{tabular}{|c|c|c|c|c|c|}
\hline \multirow{2}{*}{$\begin{array}{l}\text { Center } \\
\text { Number }\end{array}$} & \multirow{2}{*}{$\begin{array}{l}\text { Atomic } \\
\text { Number }\end{array}$} & \multirow{2}{*}{$\begin{array}{l}\text { Atomic } \\
\text { Type }\end{array}$} & \multicolumn{3}{|c|}{ Coordinates (Angstroms) } \\
\hline & & & $\mathrm{X}$ & $\mathrm{Y}$ & \\
\hline 1 & 6 & 0 & 0.352388 & 4.872908 & 0.690724 \\
\hline 2 & 6 & 0 & 0.411256 & 4.449797 & -0.635356 \\
\hline 3 & 6 & 0 & 0.409727 & 3.090338 & -0.940518 \\
\hline 4 & 6 & 0 & 0.343910 & 2.129759 & 0.073084 \\
\hline 5 & 6 & 0 & 0.280154 & 2.563676 & 1.398418 \\
\hline 6 & 6 & 0 & 0.285515 & 3.925348 & 1.706713 \\
\hline 7 & 1 & 0 & 0.466659 & 5.183585 & -1.440047 \\
\hline 8 & 1 & 0 & 0.481399 & 2.774149 & -1.982892 \\
\hline 9 & 1 & 0 & 0.213863 & 1.838313 & 2.214034 \\
\hline 10 & 1 & 0 & 0.235938 & 4.243054 & 2.748523 \\
\hline 11 & 53 & 0 & 0.442433 & -2.574802 & -1.182655 \\
\hline 12 & 46 & 0 & 0.391453 & 0.163081 & -0.429852 \\
\hline 13 & 15 & 0 & -1.911876 & -0.016065 & 0.027276 \\
\hline 14 & 6 & 0 & 3.286872 & 1.727252 & -0.789919 \\
\hline 15 & 1 & 0 & 3.264170 & 2.482064 & -0.007848 \\
\hline 16 & 6 & 0 & 2.514164 & 0.493563 & -0.830342 \\
\hline 17 & 6 & 0 & 2.877827 & -0.047553 & -2.043040 \\
\hline 18 & 1 & 0 & 2.712170 & -0.286763 & 0.319543 \\
\hline 19 & 6 & 0 & 4.024312 & 1.809904 & -1.922043 \\
\hline 20 & 8 & 0 & 3.774163 & 0.713103 & -2.710535 \\
\hline 21 & 6 & 0 & -3.007852 & -0.503637 & -1.355072 \\
\hline 22 & 6 & 0 & -2.477605 & -0.738473 & -2.624080 \\
\hline 23 & 6 & 0 & -4.392935 & -0.577124 & -1.161580 \\
\hline 24 & 6 & 0 & -3.321289 & -1.053293 & -3.685989 \\
\hline 25 & 1 & 0 & -1.398540 & -0.692892 & -2.770118 \\
\hline 26 & 6 & 0 & -5.230688 & -0.902460 & -2.220167 \\
\hline 27 & 1 & 0 & -4.816272 & -0.374935 & -0.176475 \\
\hline 28 & 6 & 0 & -4.693908 & -1.139500 & -3.484199 \\
\hline 29 & 1 & 0 & -2.898702 & -1.236854 & -4.672211 \\
\hline 30 & 1 & 0 & -6.306134 & -0.964563 & -2.061697 \\
\hline 31 & 1 & 0 & -5.351588 & -1.390154 & -4.315231 \\
\hline 32 & 6 & 0 & -2.152386 & -1.263772 & 1.342760 \\
\hline 33 & 6 & 0 & -1.549371 & -1.032612 & 2.585509 \\
\hline 34 & 6 & 0 & -2.792086 & -2.482690 & 1.115841 \\
\hline 35 & 6 & 0 & -1.610539 & -1.990383 & 3.589886 \\
\hline 36 & 1 & 0 & -1.028784 & -0.089448 & 2.766963 \\
\hline 37 & 6 & 0 & -2.838970 & -3.447228 & 2.118060 \\
\hline 38 & 1 & 0 & -3.236722 & -2.688250 & 0.143047 \\
\hline 39 & 6 & 0 & -2.252925 & -3.204146 & 3.354952 \\
\hline 40 & 1 & 0 & -1.153832 & -1.790761 & 4.558943 \\
\hline 41 & 1 & 0 & -3.331574 & -4.398670 & 1.925223 \\
\hline 42 & 1 & 0 & -2.293716 & -3.960689 & 4.136860 \\
\hline 43 & 6 & 0 & -2.782954 & 1.483602 & 0.631158 \\
\hline 44 & 6 & 0 & -2.815476 & 2.579831 & -0.239004 \\
\hline 45 & 6 & 0 & -3.373642 & 1.600716 & 1.888995 \\
\hline 46 & 6 & 0 & -3.403670 & 3.773906 & 0.150700 \\
\hline 47 & 1 & 0 & -2.359146 & 2.501037 & -1.226461 \\
\hline 48 & 6 & 0 & -3.964878 & 2.800907 & 2.278901 \\
\hline 49 & 1 & 0 & -3.379356 & 0.754265 & 2.574165 \\
\hline 50 & 6 & 0 & -3.973632 & 3.890127 & 1.416591 \\
\hline 51 & 1 & 0 & -3.405226 & 4.622015 & -0.532027 \\
\hline 52 & 1 & 0 & -4.420905 & 2.880287 & 3.264820 \\
\hline 53 & 1 & 0 & -4.427449 & 4.829813 & 1.727582 \\
\hline
\end{tabular}




\begin{tabular}{|c|c|c|c|c|c|}
\hline 54 & 6 & 0 & 4.981148 & 2.800056 & -2.463857 \\
\hline 55 & 1 & 0 & 5.099450 & 3.628032 & -1.756610 \\
\hline 56 & 1 & 0 & 4.633351 & 3.215025 & -3.419699 \\
\hline 57 & 1 & 0 & 2.589421 & -0.968928 & -2.537806 \\
\hline 58 & 1 & 0 & 5.970383 & 2.356656 & -2.642490 \\
\hline 59 & 1 & 0 & 0.355773 & 5.935496 & 0.930246 \\
\hline 60 & 7 & 0 & 3.214496 & -0.937030 & 1.367796 \\
\hline 61 & 6 & 0 & 2.049082 & -1.372024 & 2.172247 \\
\hline 62 & 1 & 0 & 1.464364 & -2.048634 & 1.533357 \\
\hline 63 & 6 & 0 & 3.933166 & -2.102602 & 0.795396 \\
\hline 64 & 1 & 0 & 3.193473 & -2.627700 & 0.173318 \\
\hline 65 & 1 & 0 & 4.222906 & -2.778292 & 1.616233 \\
\hline 66 & 6 & 0 & 4.116171 & -0.000896 & 2.071064 \\
\hline 67 & 1 & 0 & 4.807220 & 0.393397 & 1.314730 \\
\hline 68 & 1 & 0 & 4.720682 & -0.564938 & 2.802710 \\
\hline 69 & 1 & 0 & 1.414562 & -0.486802 & 2.322324 \\
\hline 70 & 6 & 0 & 3.402448 & 1.158918 & 2.735770 \\
\hline 71 & 1 & 0 & 2.814191 & 0.853493 & 3.609822 \\
\hline 72 & 1 & 0 & 4.145284 & 1.887942 & 3.080556 \\
\hline 73 & 1 & 0 & 2.726473 & 1.672915 & 2.039801 \\
\hline 74 & 6 & 0 & 5.155531 & -1.748582 & -0.029391 \\
\hline 75 & 1 & 0 & 5.993179 & -1.395117 & 0.584115 \\
\hline 76 & 1 & 0 & 5.495094 & -2.648931 & -0.554499 \\
\hline 77 & 1 & 0 & 4.933884 & -0.988608 & -0.788481 \\
\hline 78 & 6 & 0 & 2.382766 & -2.036403 & 3.494716 \\
\hline 79 & 1 & 0 & 2.940714 & -1.379544 & 4.174724 \\
\hline 80 & 1 & 0 & 1.448325 & -2.317870 & 3.995094 \\
\hline 81 & 1 & 0 & 2.962846 & -2.957465 & 3.360785 \\
\hline \multicolumn{6}{|c|}{0.668965 (Hartree/Particle) } \\
\hline Thermal & to & \multicolumn{4}{|c|}{0.710825} \\
\hline Thermal & 1 to & \multicolumn{4}{|c|}{0.711769} \\
\hline Thermal & to & \multicolumn{4}{|c|}{0.593278} \\
\hline Sum of e & and & \multicolumn{4}{|c|}{-1965.985889} \\
\hline Sum of e & and & \multicolumn{4}{|c|}{-1965.944029} \\
\hline Sum of e & and & \multicolumn{4}{|c|}{-1965.943085} \\
\hline Sum of e & and & \multicolumn{4}{|c|}{-1966.061576} \\
\hline
\end{tabular}

$1 \mathrm{~b}$

\begin{tabular}{rrrrrr} 
Center & Atomic & Atomic & \multicolumn{3}{c}{ Coordinates (Angstroms) } \\
Number & Number & Type & X & Y & $Z$ \\
--1 & 6 & 0 & -2.273333 & -0.306429 & -0.803889 \\
2 & 1 & 0 & -2.612115 & -0.208966 & -1.839251 \\
3 & 6 & 0 & -1.082867 & -0.789805 & -0.542097 \\
4 & 6 & 0 & 0.100400 & -1.272221 & -0.261971 \\
5 & 1 & 0 & 0.179933 & -2.353182 & -0.115228 \\
6 & 6 & 0 & -3.213188 & 0.117537 & 0.276781 \\
7 & 8 & 0 & -2.936555 & 0.036079 & 1.454351 \\
8 & 6 & 0 & -4.535930 & 0.651241 & -0.211048 \\
9 & 1 & 0 & -5.062642 & -0.114156 & -0.797614 \\
10 & 1 & 0 & -4.382009 & 1.510509 & -0.878093 \\
11 & 1 & 0 & -5.153465 & 0.950452 & 0.639893 \\
12 & 6 & 0 & 1.341983 & -0.503698 & -0.124220 \\
13 & 6 & 0 & 2.532593 & -1.177216 & 0.161277 \\
14 & 6 & 0 & 1.371076 & 0.887482 & -0.274010 \\
15 & 6 & 0 & 3.727012 & -0.479409 & 0.293040 \\
16 & 1 & 0 & 2.514866 & -2.260703 & 0.281066 \\
17 & 6 & 0 & 2.563312 & 1.582517 & -0.143198 \\
18 & 1 & 0 & 0.443883 & 1.417897 & -0.490300 \\
19 & 6 & 0 & 3.746392 & 0.902133 & 0.140192 \\
20 & 1 & 0 & 4.645991 & -1.018375 & 0.516982 \\
21 & 1 & 0 & 2.572182 & 2.664792 & -0.260548 \\
22 & 1 & 0 & 4.681120 & 1.450307 & 0.243155
\end{tabular}

Zero-point correction

0.175265 (Hartree/Particle)

Thermal correction to Energy=

to Enthalpy=

0.186493

Thermal correction to Gibbs Free Energy=

Sum of electronic and zero-point Energies-

Sum of electronic and thermal Energies =

Sum of electronic and thermal Enthalpies=

0.187437

0.136380

$-499.815643$

$-499.804415$

$-499.803471$

Sum of electronic and thermal Free Energies= $\quad-499.854528$

M06 /6-311++G(d,p)-SDD/SMD//M06/6-31G(d)-LANL2DZ energy in toluene solvent $=-500.1307302$

$1 \mathrm{c}$

\begin{tabular}{|c|c|c|c|c|c|}
\hline \multirow{2}{*}{$\begin{array}{l}\text { Center } \\
\text { Number }\end{array}$} & \multirow{2}{*}{$\begin{array}{l}\text { Atomic } \\
\text { Number }\end{array}$} & \multirow{2}{*}{$\begin{array}{l}\text { Atomic } \\
\text { Type }\end{array}$} & \multicolumn{3}{|c|}{ Coordinates (Angstroms) } \\
\hline & & & $\mathrm{X}$ & $\mathrm{Y}$ & $\mathrm{Z}$ \\
\hline 1 & 6 & 0 & 3.235991 & 0.274570 & -0.752869 \\
\hline 2 & 1 & 0 & 3.599218 & 0.223241 & -1.783635 \\
\hline 3 & 6 & 0 & 2.084605 & 0.843774 & -0.486108 \\
\hline 4 & 6 & 0 & 0.940952 & 1.413060 & -0.202531 \\
\hline 5 & 1 & 0 & 0.958476 & 2.483725 & 0.021405 \\
\hline 6 & 6 & 0 & 4.101523 & -0.311421 & 0.313050 \\
\hline 7 & 8 & 0 & 3.793968 & -0.306034 & 1.486003 \\
\hline 8 & 6 & 0 & 5.395028 & -0.907484 & -0.182237 \\
\hline 9 & 1 & 0 & 5.999287 & -0.140294 & -0.685845 \\
\hline 10 & 1 & 0 & 5.198252 & -1.692316 & -0.925440 \\
\hline 11 & 1 & 0 & 5.958093 & -1.326185 & 0.655965 \\
\hline
\end{tabular}




$\begin{array}{rrrrrr}12 & 6 & 0 & -0.369356 & 0.761839 & -0.151766 \\ 13 & 6 & 0 & -1.504773 & 1.516207 & 0.137311 \\ 14 & 6 & 0 & -0.529549 & -0.612539 & -0.387396 \\ 15 & 6 & 0 & -2.769885 & 0.938938 & 0.193091 \\ 16 & 1 & 0 & -1.399263 & 2.585126 & 0.324748 \\ 17 & 6 & 0 & -1.776459 & -1.198998 & -0.336318 \\ 18 & 1 & 0 & 0.347693 & -1.219684 & -0.609784 \\ 19 & 6 & 0 & -2.908793 & -0.427190 & -0.045153 \\ 20 & 1 & 0 & -3.632115 & 1.559270 & 0.423703 \\ 21 & 1 & 0 & -1.912580 & -2.263740 & -0.514460 \\ 22 & 8 & 0 & -4.085767 & -1.097058 & -0.017530 \\ 23 & 6 & 0 & -5.247227 & -0.362840 & 0.280008 \\ 24 & 1 & 0 & -6.077276 & -1.073195 & 0.254973 \\ 25 & 1 & 0 & -5.191611 & 0.093074 & 1.280013 \\ 26 & 1 & 0 & -5.426131 & 0.428216 & -0.463929\end{array}$

Zero-point correction-

0.207920 (Hartree/Particle)

Thermal correction to Energy=

Thermal correction to Enthalpy=

Thermal correction to Gibbs Free Energy=

Sum of electronic and zero-point Energies

Sum of electronic and thermal Energies $=$

Sum of electronic and thermal Enthalpies=

0.222665

0.165582

$-614.246797$

$-614.232995$

$-614.232051$

Sum of electronic and thermal Free Energies=

$\mathrm{M} 06 / 6-311++\mathrm{G}(\mathrm{d}, \mathrm{p})-\mathrm{SDD} / \mathrm{SMD} / / \mathrm{M} 06 / 6-31 \mathrm{G}(\mathrm{d})-\mathrm{LANL} 2 \mathrm{DZ}$ energy in toluene solvent $=-614.6248132$

1d

\begin{tabular}{rrrrrr}
$\begin{array}{l}\text { Center } \\
\text { Number }\end{array}$ & $\begin{array}{c}\text { Atomic } \\
\text { Number }\end{array}$ & $\begin{array}{c}\text { Atomic } \\
\text { Type }\end{array}$ & \multicolumn{2}{c}{ Coordinates (Angstroms) } \\
------------- & & \\
1 & 6 & 0 & 3.461032 & 0.203033 & -0.773464 \\
2 & 1 & 0 & 3.811047 & 0.118089 & -1.805663 \\
3 & 6 & 0 & 2.353517 & 0.847939 & -0.506268 \\
4 & 6 & 0 & 1.254580 & 1.497955 & -0.216749 \\
5 & 1 & 0 & 1.337468 & 2.568350 & -0.010526 \\
6 & 6 & 0 & 4.293076 & -0.420396 & 0.304867 \\
7 & 8 & 0 & 3.964001 & -0.399084 & 1.470289 \\
8 & 6 & 0 & 5.566217 & -1.064642 & -0.175103 \\
9 & 1 & 0 & 6.211230 & -0.317392 & -0.657731 \\
10 & 1 & 0 & 5.349823 & -1.831248 & -0.931559 \\
11 & 1 & 0 & 6.096035 & -1.516129 & 0.667501 \\
12 & 6 & 0 & -0.088736 & 0.919235 & -0.143862 \\
13 & 6 & 0 & -1.175073 & 1.759476 & 0.127062 \\
14 & 6 & 0 & -0.317401 & -0.450335 & -0.339642 \\
15 & 6 & 0 & -2.463296 & 1.255114 & 0.198451 \\
16 & 1 & 0 & -1.001015 & 2.823282 & 0.283419 \\
17 & 6 & 0 & -1.597567 & -0.967593 & -0.271083 \\
18 & 1 & 0 & 0.526375 & -1.108082 & -0.542464 \\
19 & 6 & 0 & -2.655173 & -0.104232 & -0.003728 \\
20 & 1 & 0 & -3.318098 & 1.890847 & 0.408372 \\
21 & 1 & 0 & -1.797629 & -2.025031 & -0.418141 \\
22 & 7 & 0 & -4.014378 & -0.648151 & 0.069538 \\
23 & 8 & 0 & -4.152654 & -1.848439 & -0.107332 \\
24 & 8 & 0 & -4.924052 & 0.132652 & 0.301686
\end{tabular}

Zero-point correction $=$

Thermal correction to Energy=

0.178184 (Hartree/Particle)

Thermal correction to Enthalpy-

Thermal correction to Gibbs Free Energy=

Sum of electronic and zero-point Energies=

Sum of electronic and thermal Energies $=$

Sum of electronic and thermal Enthalpies=

Sum of electronic and thermal Free Energies

0.191973

0.192918

0.134777

$-704.229711$

$-704.21592$

$-704.214977$

M06 /6-311++G(d,p)-SDD/SMD//M06/6-31G(d)-LANL2DZ energy in toluene solvent $=-704.6028695$

\begin{tabular}{|c|c|c|c|c|c|}
\hline \multirow{2}{*}{$\begin{array}{l}\text { Center } \\
\text { Number }\end{array}$} & \multirow{2}{*}{$\begin{array}{l}\text { Atomic } \\
\text { Number }\end{array}$} & \multirow{2}{*}{$\begin{array}{l}\text { Atomic } \\
\text { Type }\end{array}$} & \multicolumn{3}{|c|}{ Coordinates (Angstroms) } \\
\hline & & & $\mathrm{X}$ & $\mathrm{Y}$ & \\
\hline 1 & 6 & 0 & 0.392235 & -0.826366 & -0.058306 \\
\hline 2 & 1 & 0 & 0.668027 & -1.870946 & 0.107655 \\
\hline 3 & 6 & 0 & -0.859126 & -0.502378 & -0.291834 \\
\hline 4 & 6 & 0 & -2.092868 & -0.156100 & -0.522900 \\
\hline 5 & 1 & 0 & -2.428556 & -0.129345 & -1.562805 \\
\hline 6 & 6 & 0 & 1.474118 & 0.196738 & -0.009867 \\
\hline 7 & 8 & 0 & 1.267730 & 1.381335 & -0.173466 \\
\hline 8 & 6 & 0 & 2.852763 & -0.352769 & 0.257942 \\
\hline 9 & 1 & 0 & 3.136119 & -1.071503 & -0.523311 \\
\hline 10 & 1 & 0 & 2.870423 & -0.898884 & 1.211095 \\
\hline 11 & 1 & 0 & 3.579903 & 0.462886 & 0.287699 \\
\hline 12 & 6 & 0 & -3.086334 & 0.221766 & 0.534042 \\
\hline 13 & 1 & 0 & -3.441243 & 1.248824 & 0.379638 \\
\hline 14 & 1 & 0 & -3.965317 & -0.435102 & 0.499071 \\
\hline 15 & 1 & 0 & -2.645918 & 0.158047 & 1.534223 \\
\hline
\end{tabular}

Zero-point correction $=$

0.121959 (Hartree/Particle)

Thermal correction to Energy=

0.130454

Thermal correction to Enthalpy=

Thermal correction to Gibbs Free Energy=

Sum of electronic and zero-point Energies

Sum of electronic and thermal Energies=

0.131398

0.088302

$-308.275387$

$-308.26689$ 


\begin{tabular}{|c|c|c|c|c|c|}
\hline \multirow{2}{*}{$\begin{array}{l}\text { Center } \\
\text { Number }\end{array}$} & \multirow{2}{*}{$\begin{array}{l}\text { Atomic } \\
\text { Number }\end{array}$} & \multirow{2}{*}{$\begin{array}{l}\text { Atomic } \\
\text { Type }\end{array}$} & \multicolumn{3}{|c|}{ Coordinates (Angstroms) } \\
\hline & & & $\mathrm{X}$ & $\mathrm{Y}$ & \\
\hline 1 & 6 & 0 & 1.565785 & -0.939543 & -0.179935 \\
\hline 2 & 1 & 0 & 1.906193 & -1.950901 & 0.054356 \\
\hline 3 & 6 & 0 & 0.325750 & -0.714476 & -0.551816 \\
\hline 4 & 6 & 0 & -0.891309 & -0.411582 & -0.896522 \\
\hline 5 & 1 & 0 & -1.161489 & -0.440480 & -1.957302 \\
\hline 6 & 6 & 0 & 2.538670 & 0.180308 & -0.050023 \\
\hline 7 & 8 & 0 & 2.217517 & 1.344329 & -0.179717 \\
\hline 8 & 6 & 0 & 3.951264 & -0.235596 & 0.273255 \\
\hline 9 & 1 & 0 & 4.334840 & -0.925154 & -0.491114 \\
\hline 10 & 1 & 0 & 3.979028 & -0.776826 & 1.229132 \\
\hline 11 & 1 & 0 & 4.595528 & 0.645539 & 0.332749 \\
\hline 12 & 6 & 0 & -1.984161 & 0.026827 & 0.059625 \\
\hline 13 & 6 & 0 & -2.484837 & 1.392891 & -0.412916 \\
\hline 14 & 1 & 0 & -3.298742 & 1.746473 & 0.236105 \\
\hline 15 & 1 & 0 & -1.675829 & 2.134710 & -0.388564 \\
\hline 16 & 1 & 0 & -2.871285 & 1.343200 & -1.440854 \\
\hline 17 & 6 & 0 & -3.123926 & -0.990556 & 0.000861 \\
\hline 18 & 1 & 0 & -3.954418 & -0.674279 & 0.648287 \\
\hline 19 & 1 & 0 & -3.515510 & -1.093386 & -1.021161 \\
\hline 20 & 1 & 0 & -2.786641 & -1.980536 & 0.336013 \\
\hline 21 & 6 & 0 & -1.452830 & 0.141538 & 1.482953 \\
\hline 22 & 1 & 0 & -1.091382 & -0.826951 & 1.855035 \\
\hline 23 & 1 & 0 & -0.619661 & 0.856463 & 1.534431 \\
\hline 24 & 1 & 0 & -2.247221 & 0.488623 & 2.157733 \\
\hline
\end{tabular}

Thermal correction to Energy=

0.219005

Thermal correction to Enthalpy=

0.219949

Thermal correction to Gibbs Free Energy=

Sum of electronic and zero-point Energies=

Sum of electronic and thermal Energies=
Sum of electronic and thermal Enthalpies

0.168651

0.168651

$-426.039211$

Sum of electronic and thermal Free Energies=

$-426.026241$

$1 \mathrm{~g}$

\begin{tabular}{|c|c|c|c|c|c|}
\hline \multirow{2}{*}{$\begin{array}{l}\text { Center } \\
\text { Number }\end{array}$} & \multirow{2}{*}{$\begin{array}{l}\text { Atomic } \\
\text { Number }\end{array}$} & \multirow{2}{*}{$\begin{array}{l}\text { Atomic } \\
\text { Type }\end{array}$} & \multicolumn{3}{|c|}{ Coordinates (Angstroms) } \\
\hline & & & $\mathrm{X}$ & $\mathrm{Y}$ & Z \\
\hline 1 & 6 & 0 & 1.513436 & -0.860358 & -0.281647 \\
\hline 2 & 1 & 0 & 1.793912 & -1.908444 & -0.159528 \\
\hline 3 & 6 & 0 & 0.287242 & -0.548821 & -0.612614 \\
\hline 4 & 6 & 0 & -0.927977 & -0.216634 & -0.938327 \\
\hline 5 & 1 & 0 & -1.228134 & -0.091691 & -1.978475 \\
\hline 6 & 6 & 0 & 2.551967 & 0.193288 & -0.046156 \\
\hline 7 & 8 & 0 & 2.328456 & 1.369189 & -0.228221 \\
\hline 8 & 6 & 0 & 3.879988 & -0.326200 & 0.433763 \\
\hline 9 & 1 & 0 & 4.282584 & -1.066881 & -0.270531 \\
\hline 10 & 1 & 0 & 3.760115 & -0.837625 & 1.398864 \\
\hline 11 & 1 & 0 & 4.585663 & 0.501084 & 0.543761 \\
\hline 12 & 6 & 0 & -2.000215 & 0.026726 & 0.074877 \\
\hline 13 & 9 & 0 & -2.434791 & 1.287719 & 0.014783 \\
\hline 14 & 9 & 0 & -3.053768 & -0.763329 & -0.163867 \\
\hline 15 & 9 & 0 & -1.583488 & -0.208608 & 1.317118 \\
\hline
\end{tabular}

Zero-point correction $=$

0.099933 (Hartree/Particle)

Thermal correction to Energy=

0.109985

Thermal correction to Enthalpy=

Thermal correction to Gibbs Free Energy=

Sum of electronic and zero-point Energies

Sum of electronic and thermal Energies=

0.062772

Sum of electronic and thermal Enthalpies=

Sum of electronic and thermal Free Energies=

$-605.949139$

$-605.939087$

$-605.938143$

M06 /6-311++G(d,p)-SDD/SMD//M06/6-31G(d)-LANL2DZ energy in toluene solvent $=-606.2263508$

1h

\begin{tabular}{|c|c|c|c|c|c|}
\hline \multirow{2}{*}{$\begin{array}{l}\text { Center } \\
\text { Number }\end{array}$} & \multirow{2}{*}{$\begin{array}{l}\text { Atomic } \\
\text { Number }\end{array}$} & \multirow{2}{*}{$\begin{array}{l}\text { Atomic } \\
\text { Type }\end{array}$} & \multicolumn{3}{|c|}{ Coordinates (Angstroms) } \\
\hline & & & $\mathrm{X}$ & $\mathrm{Y}$ & Z \\
\hline 1 & 6 & 0 & -0.235514 & 0.446395 & -0.007238 \\
\hline 2 & 6 & 0 & -1.474038 & -0.000939 & 0.004301 \\
\hline 3 & 6 & 0 & -2.696906 & -0.439095 & 0.018949 \\
\hline 4 & 1 & 0 & -3.221311 & -0.633383 & 0.954358 \\
\hline 5 & 6 & 0 & 0.872641 & -0.564330 & -0.007395 \\
\hline 6 & 8 & 0 & 0.657045 & -1.758942 & -0.029066 \\
\hline 7 & 6 & 0 & 2.280461 & -0.021657 & 0.028635 \\
\hline 8 & 1 & 0 & 2.460650 & 0.534319 & 0.959008 \\
\hline 9 & 1 & 0 & 2.465140 & 0.676655 & -0.798250 \\
\hline 10 & 1 & 0 & -3.238578 & -0.648322 & -0.903263 \\
\hline 11 & 1 & 0 & 2.983795 & -0.856249 & -0.031964 \\
\hline 12 & 6 & 0 & 0.064436 & 1.922257 & -0.017920 \\
\hline 13 & 1 & 0 & 0.655694 & 2.224062 & 0.857574 \\
\hline
\end{tabular}




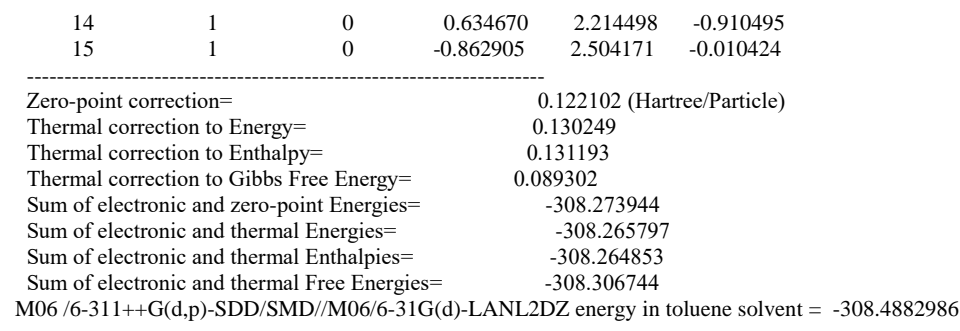

1i

\begin{tabular}{rrrrrr} 
Center & $\begin{array}{c}\text { Atomic } \\
\text { Number }\end{array}$ & Number & $\begin{array}{c}\text { Atomic } \\
\text { Type }\end{array}$ & \multicolumn{2}{c}{ Coordinates (Angstroms) } \\
-1 & 6 & 0 & 0.994535 & 0.236469 & -0.077189 \\
\hline 1 & 6 & 0 & 1.596414 & 1.399877 & -0.226302 \\
3 & 6 & 0 & 2.195444 & 2.545073 & -0.356649 \\
4 & 1 & 0 & 2.482581 & 2.925574 & -1.336584 \\
5 & 6 & 0 & 1.873633 & -0.969911 & 0.132382 \\
6 & 8 & 0 & 3.042815 & -0.963650 & -0.189735 \\
7 & 6 & 0 & 1.249031 & -2.162566 & 0.813256 \\
8 & 1 & 0 & 0.582785 & -2.703957 & 0.129949 \\
9 & 1 & 0 & 0.637106 & -1.860549 & 1.672779 \\
10 & 1 & 0 & 2.448723 & 3.160619 & 0.506268 \\
11 & 1 & 0 & 2.047139 & -2.838341 & 1.133093 \\
12 & 6 & 0 & -0.487787 & 0.143890 & -0.057083 \\
13 & 6 & 0 & -1.154262 & -0.774328 & -0.875010 \\
14 & 6 & 0 & -1.244445 & 0.989672 & 0.757863 \\
15 & 6 & 0 & -2.541285 & -0.850360 & -0.870779 \\
16 & 1 & 0 & -0.576391 & -1.418688 & -1.538389 \\
17 & 6 & 0 & -2.632116 & 0.915102 & 0.761610 \\
18 & 1 & 0 & -0.728919 & 1.708376 & 1.394329 \\
19 & 6 & 0 & -3.284494 & -0.007524 & -0.049793 \\
20 & 1 & 0 & -3.044868 & -1.566640 & -1.517707 \\
21 & 1 & 0 & -3.207461 & 1.578813 & 1.404928 \\
22 & 1 & 0 & -4.371215 & -0.068374 & -0.044607
\end{tabular}

Zero-point correction=

Thermal correction to Energy=

0.175014 (Hartree/Particle)

Thermal correction to Enthalpy=

0.186125

Thermal correction to Gibbs Free Energy=

Sum of electronic and zero-point Energies $=$

Sum of electronic and thermal Energies=

Sum of electronic and thermal Enthalpies=

Sum of electronic and thermal Free Energies=

0.137397

$-499.809975$

$-499.798864$

-499.797920
-499.847592

M06 /6-311++G(d,p)-SDD/SMD//M06/6-31G(d)-LANL2DZ energy in toluene solvent $=-500.1235885$

$\mathbf{1 j}$

\begin{tabular}{|c|c|c|c|c|c|}
\hline \multirow{2}{*}{$\begin{array}{l}\text { Center } \\
\text { Number }\end{array}$} & \multirow{2}{*}{$\begin{array}{l}\text { Atomic } \\
\text { Number }\end{array}$} & \multirow{2}{*}{$\begin{array}{l}\text { Atomic } \\
\text { Type }\end{array}$} & \multicolumn{3}{|c|}{ Coordinates (Angstroms) } \\
\hline & & & $\mathrm{X}$ & $\mathrm{Y}$ & $\mathrm{z}$ \\
\hline 1 & 6 & 0 & 0.354464 & 0.227956 & -0.006601 \\
\hline 2 & 6 & 0 & 0.957348 & 1.397198 & -0.025301 \\
\hline 3 & 6 & 0 & 1.579008 & 2.533482 & -0.048565 \\
\hline 4 & 1 & 0 & 1.847594 & 3.012649 & -0.988839 \\
\hline 5 & 6 & 0 & 1.188801 & -1.028190 & 0.014363 \\
\hline 6 & 8 & 0 & 2.395303 & -0.950265 & 0.091154 \\
\hline 7 & 6 & 0 & 0.477754 & -2.349477 & -0.082269 \\
\hline 8 & 1 & 0 & -0.035717 & -2.435942 & -1.047771 \\
\hline 9 & 1 & 0 & -0.290000 & -2.451091 & 0.693594 \\
\hline 10 & 1 & 0 & 1.856368 & 3.044139 & 0.872402 \\
\hline 11 & 1 & 0 & 1.216253 & -3.149080 & 0.015806 \\
\hline 12 & 6 & 0 & -1.145677 & 0.172520 & 0.011624 \\
\hline 13 & 9 & 0 & -1.693394 & 1.383625 & -0.056028 \\
\hline 14 & 9 & 0 & -1.624610 & -0.543990 & -1.016130 \\
\hline 15 & 9 & 0 & -1.596119 & -0.410689 & 1.132833 \\
\hline
\end{tabular}

Zero-point correction $=\quad 0.100170$ (Hartree/Particle)

Thermal correction to Energy=

0.109923

Thermal correction to Enthalpy=

Thermal correction to Gibbs Free Energy=

Sum of electronic and zero-point Energies

Sum of electronic and thermal Energies=

0.110867
0.064318

$-605.950019$

$-605.940265$

Sum of electronic and thermal Enthalpies=
Sum of electronic and thermal Free Energie

$-605.939321$

M06 /6-311++G(d,p)-SDD/SMD//M06/6-31G(d)-LANL2DZ energy in toluene solvent $=-606.2238007$

$1 \mathrm{k}$

\begin{tabular}{cccccc}
$\begin{array}{l}\text { Center } \\
\text { Number }\end{array}$ & $\begin{array}{c}\text { Atomic } \\
\text { Number }\end{array}$ & $\begin{array}{c}\text { Atomic } \\
\text { Type }\end{array}$ & \multicolumn{4}{c}{ Coordinates (Angstroms) } & X & Y & Z \\
- \hdashline 1 & 6 & 0 & 2.757767 & -0.655845 & -0.197808 \\
2 & 1 & 0 & 2.407214 & -1.649906 & -0.474112 \\
3 & 6 & 0 & 4.047009 & -0.456240 & -0.050804 \\
4 & 6 & 0 & 5.320696 & -0.255033 & 0.103774 \\
5 & 1 & 0 & 5.803279 & -0.382579 & 1.072021 \\
6 & 6 & 0 & 1.784756 & 0.457782 & -0.022373
\end{tabular}




\begin{tabular}{|c|c|c|c|c|c|}
\hline 7 & 8 & 0 & 2.164398 & 1.610372 & 0.106084 \\
\hline 8 & 1 & 0 & 5.948712 & 0.062813 & -0.727537 \\
\hline 9 & 6 & 0 & 0.336738 & 0.124473 & -0.020450 \\
\hline 10 & 6 & 0 & -0.172114 & -1.180464 & 0.037204 \\
\hline 11 & 6 & 0 & -0.570813 & 1.185892 & -0.052825 \\
\hline 12 & 6 & 0 & -1.533881 & -1.412360 & 0.057604 \\
\hline 13 & 1 & 0 & 0.498945 & -2.035878 & 0.087673 \\
\hline 14 & 6 & 0 & -1.940030 & 0.971045 & -0.041923 \\
\hline 15 & 1 & 0 & -0.168345 & 2.196453 & -0.087321 \\
\hline 16 & 6 & 0 & -2.428418 & -0.337573 & 0.015548 \\
\hline 17 & 1 & 0 & -1.941446 & -2.419541 & 0.110390 \\
\hline 18 & 1 & 0 & -2.616452 & 1.820977 & -0.075194 \\
\hline 19 & 8 & 0 & -3.738595 & -0.664497 & 0.038690 \\
\hline 20 & 6 & 0 & -4.683974 & 0.378450 & -0.000421 \\
\hline 21 & 1 & 0 & -4.606938 & 0.958165 & -0.931956 \\
\hline 22 & 1 & 0 & -5.666655 & -0.096926 & 0.046287 \\
\hline 23 & 1 & 0 & -4.571152 & 1.058654 & 0.856396 \\
\hline \multicolumn{4}{|c|}{ Zero-point correction= } & \multicolumn{2}{|c|}{0.179783 (Hartree/Particle } \\
\hline Thermal & to & & \multicolumn{3}{|c|}{0.191196} \\
\hline Thermal & to & & \multicolumn{3}{|c|}{0.192140} \\
\hline Thermal & to & Energy $=$ & \multicolumn{3}{|c|}{0.141830} \\
\hline Sum of & and & Energies $=$ & \multicolumn{3}{|c|}{-574.989987} \\
\hline Sum of & and & ergies $=$ & \multicolumn{3}{|c|}{$\begin{array}{l}-574.978574 \\
-574077630\end{array}$} \\
\hline Sum of & and & thalpies $=$ & \multicolumn{3}{|c|}{-574.977630} \\
\hline Sum of & and & ee Energies & \multicolumn{3}{|c|}{-575.027940} \\
\hline
\end{tabular}

M06 /6-311++G(d,p)-SDD/SMD//M06/6-31G(d)-LANL2DZ energy in toluene solvent = -575.3272462

11

\begin{tabular}{|c|c|c|c|c|c|}
\hline \multirow{2}{*}{$\begin{array}{l}\text { Center } \\
\text { Number }\end{array}$} & \multirow{2}{*}{$\begin{array}{l}\text { Atomic } \\
\text { Number }\end{array}$} & \multirow{2}{*}{$\begin{array}{l}\text { Atomic } \\
\text { Type }\end{array}$} & \multicolumn{3}{|c|}{ Coordinates (Angstroms) } \\
\hline & & & $\mathrm{X}$ & $\mathrm{Y}$ & Z \\
\hline 1 & 6 & 0 & 2.958259 & -0.692585 & -0.271544 \\
\hline 2 & 1 & 0 & 2.533250 & -1.622336 & -0.649485 \\
\hline 3 & 6 & 0 & 4.259352 & -0.599778 & -0.109345 \\
\hline 4 & 6 & 0 & 5.541528 & -0.501779 & 0.062985 \\
\hline 5 & 1 & 0 & 6.009267 & -0.762706 & 1.011444 \\
\hline 6 & 6 & 0 & 2.073090 & 0.462083 & 0.016728 \\
\hline 7 & 8 & 0 & 2.513253 & 1.572488 & 0.250923 \\
\hline 8 & 1 & 0 & 6.193224 & -0.142501 & -0.732332 \\
\hline 9 & 6 & 0 & 0.591971 & 0.229994 & -0.002503 \\
\hline 10 & 6 & 0 & 0.016438 & -1.040589 & 0.083653 \\
\hline 11 & 6 & 0 & -0.232513 & 1.357476 & -0.075002 \\
\hline 12 & 6 & 0 & -1.363199 & -1.190411 & 0.091218 \\
\hline 13 & 1 & 0 & 0.640567 & -1.927316 & 0.169932 \\
\hline 14 & 6 & 0 & -1.610283 & 1.224491 & -0.081390 \\
\hline 15 & 1 & 0 & 0.237188 & 2.336958 & -0.125560 \\
\hline 16 & 6 & 0 & -2.150959 & -0.052470 & 0.002389 \\
\hline 17 & 1 & 0 & -1.834602 & -2.165473 & 0.166785 \\
\hline 18 & 1 & 0 & -2.272499 & 2.082516 & -0.145325 \\
\hline 19 & 7 & 0 & -3.615734 & -0.202239 & 0.000480 \\
\hline 20 & 8 & 0 & -4.283450 & 0.815630 & -0.073406 \\
\hline 21 & 8 & 0 & -4.067099 & -1.333374 & 0.072238 \\
\hline
\end{tabular}

Zero-point correction=

Thermal correction to Energy=

Thermal correction to Enthalpy=

0.149864 (Hartree/Particle)

Thermal correction to Gibbs Free Energy

Sum of electronic and zero-point Energies=

Sum of electronic and thermal Energies=

Sum of electronic and thermal Enthalpies=

Sum of electronic and thermal Free Energies=

0.162079

0.163023

0.109431

$-664.968662$

$-664.956447$

$-664.9565503$

$-665.009095$

M06 /6-311++G(d,p)-SDD/SMD//M06/6-31G(d)-LANL2DZ energy in toluene solvent $=-665.3001928$

$1 \mathrm{~m}$

\begin{tabular}{rrrrrr}
$\begin{array}{l}\text { Center } \\
\text { Number }\end{array}$ & $\begin{array}{c}\text { Atomic } \\
\text { Number }\end{array}$ & $\begin{array}{c}\text { Atomic } \\
\text { Type }\end{array}$ & \multicolumn{2}{c}{ Coordinates (Angstroms) } \\
- \\
-1 & 6 & 0 & 1.118136 & -0.632064 & -0.001554 \\
2 & 1 & 0 & 0.772277 & -1.666147 & -0.002599 \\
3 & 6 & 0 & 2.402364 & -0.350321 & -0.000643 \\
4 & 6 & 0 & 3.664749 & -0.054342 & 0.001048 \\
5 & 1 & 0 & 4.213525 & 0.078357 & 0.932448 \\
6 & 6 & 0 & 0.110251 & 0.441934 & -0.000857 \\
7 & 8 & 0 & 0.326656 & 1.628759 & -0.000721 \\
8 & 6 & 0 & -1.335232 & -0.088273 & 0.000167 \\
9 & 1 & 0 & 4.215480 & 0.080444 & -0.928897 \\
10 & 9 & 0 & -1.531736 & -0.856222 & -1.078425 \\
11 & 9 & 0 & -2.223974 & 0.885427 & -0.000292 \\
12 & 9 & 0 & -1.530527 & -0.854130 & 1.080478
\end{tabular}

Zero-point correction=

Thermal correction to Energy=

0.071490 (Hartree/Particle)

Thermal correction to Enthalpy=

0.080163

Thermal correction to Gibbs Free Energy=

Sum of electronic and zero-point Energies=

Sum of electronic and thermal Energies=

Sum of electronic and thermal Enthalpies=

0.081107
0.036359

$-566.686395$

$-566.677723$

$-566.676778$

$-566.721526$

$\begin{array}{cc}\text { Sum of electronic and thermal Free Energies }= & -566.721526 \\ \text { M06 /6-311++G(d,p)-SDD/SMD//M06/6-31G(d)-LANL2DZ energy in toluene solvent }=-566.9213783\end{array}$ 
TS2ba

\begin{tabular}{|c|c|c|c|c|c|}
\hline \multirow{2}{*}{$\begin{array}{l}\text { Center } \\
\text { Number }\end{array}$} & \multirow{2}{*}{$\begin{array}{l}\text { Atomic } \\
\text { Number }\end{array}$} & \multirow{2}{*}{$\begin{array}{l}\text { Atomic } \\
\text { Type }\end{array}$} & \multicolumn{3}{|c|}{ Coordinates (Angstroms) } \\
\hline & & & $\mathrm{X}$ & $\mathrm{Y}$ & $\mathrm{Z}$ \\
\hline 1 & 6 & 0 & -1.097770 & 4.579993 & 0.307703 \\
\hline 2 & 6 & 0 & -1.309923 & 3.655472 & 1.323862 \\
\hline 3 & 6 & 0 & -1.356974 & 2.291096 & 1.039645 \\
\hline 4 & 6 & 0 & -1.186594 & 1.841817 & -0.273244 \\
\hline 5 & 6 & 0 & -0.930739 & 2.776485 & -1.286972 \\
\hline 6 & 6 & 0 & -0.896851 & 4.134971 & -0.999035 \\
\hline 7 & 1 & 0 & -1.432622 & 3.993016 & 2.352495 \\
\hline 8 & 1 & 0 & -1.522100 & 1.577367 & 1.847537 \\
\hline 9 & 1 & 0 & -0.778329 & 2.431764 & -2.311281 \\
\hline 10 & 1 & 0 & -0.705587 & 4.851992 & -1.797228 \\
\hline 11 & 53 & 0 & 0.047252 & -2.689635 & -1.481870 \\
\hline 12 & 46 & 0 & -0.569732 & -0.149832 & -0.619812 \\
\hline 13 & 15 & 0 & 1.708609 & 0.223984 & 0.081319 \\
\hline 14 & 6 & 0 & -3.479820 & 1.221697 & -1.700260 \\
\hline 15 & 1 & 0 & -3.202086 & 2.171226 & -2.154676 \\
\hline 16 & 6 & 0 & -2.634509 & 0.503221 & -0.954269 \\
\hline 17 & 6 & 0 & -2.671019 & -0.777261 & -0.403353 \\
\hline 18 & 1 & 0 & -2.792888 & -1.605454 & -1.104811 \\
\hline 19 & 6 & 0 & -4.872146 & 0.737959 & -1.868425 \\
\hline 20 & 8 & 0 & -5.294722 & -0.269844 & -1.331267 \\
\hline 21 & 6 & 0 & 2.060895 & -1.098135 & 1.293176 \\
\hline 22 & 6 & 0 & 3.210389 & -1.885220 & 1.246102 \\
\hline 23 & 6 & 0 & 1.082566 & -1.368290 & 2.258268 \\
\hline 24 & 6 & 0 & 3.384982 & -2.921335 & 2.159394 \\
\hline 25 & 1 & 0 & 3.965101 & -1.706774 & 0.481116 \\
\hline 26 & 6 & 0 & 1.265597 & -2.393520 & 3.176261 \\
\hline 27 & 1 & 0 & 0.156945 & -0.784925 & 2.274586 \\
\hline 28 & 6 & 0 & 2.417991 & -3.174403 & 3.125279 \\
\hline 29 & 1 & 0 & 4.280116 & -3.538879 & 2.108486 \\
\hline 30 & 1 & 0 & 0.496061 & -2.592626 & 3.921005 \\
\hline 31 & 1 & 0 & 2.556221 & -3.987915 & 3.835551 \\
\hline 32 & 6 & 0 & 2.044316 & 1.776625 & 0.998281 \\
\hline 33 & 6 & 0 & 2.148372 & 1.827226 & 2.390141 \\
\hline 34 & 6 & 0 & 2.129987 & 2.965742 & 0.265815 \\
\hline 35 & 6 & 0 & 2.333392 & 3.046409 & 3.036476 \\
\hline 36 & 1 & 0 & 2.094299 & 0.910746 & 2.975963 \\
\hline 37 & 6 & 0 & 2.316299 & 4.179739 & 0.912762 \\
\hline 38 & 1 & 0 & 2.041356 & 2.940480 & -0.821270 \\
\hline 39 & 6 & 0 & 2.415035 & 4.223358 & 2.301317 \\
\hline 40 & 1 & 0 & 2.419373 & 3.072170 & 4.121711 \\
\hline 41 & 1 & 0 & 2.373036 & 5.098042 & 0.329737 \\
\hline 42 & 1 & 0 & 2.559186 & 5.176364 & 2.808122 \\
\hline 43 & 6 & 0 & 3.061382 & 0.201912 & -1.144184 \\
\hline 44 & 6 & 0 & 4.360704 & 0.584351 & -0.786364 \\
\hline 45 & 6 & 0 & 2.794348 & -0.177895 & -2.461017 \\
\hline 46 & 6 & 0 & 5.378752 & 0.568483 & -1.731026 \\
\hline 47 & 1 & 0 & 4.570712 & 0.900257 & 0.236489 \\
\hline 48 & 6 & 0 & 3.816068 & -0.185757 & -3.406624 \\
\hline 49 & 1 & 0 & 1.785009 & -0.484244 & -2.736539 \\
\hline 50 & 6 & 0 & 5.105739 & 0.183578 & -3.042447 \\
\hline 51 & 1 & 0 & 6.387729 & 0.862006 & -1.445986 \\
\hline 52 & 1 & 0 & 3.599516 & -0.485036 & -4.430453 \\
\hline 53 & 1 & 0 & 5.904201 & 0.175171 & -3.782810 \\
\hline 54 & 6 & 0 & -5.741462 & 1.598452 & -2.750690 \\
\hline 55 & 1 & 0 & -5.283350 & 1.723528 & -3.741199 \\
\hline 56 & 1 & 0 & -5.841542 & 2.603686 & -2.318089 \\
\hline 57 & 1 & 0 & -1.068302 & 5.644790 & 0.533913 \\
\hline 58 & 1 & 0 & -6.731068 & 1.145748 & -2.855981 \\
\hline 59 & 6 & 0 & -2.865394 & -1.059593 & 1.021866 \\
\hline 60 & 6 & 0 & -2.482253 & -2.297113 & 1.558574 \\
\hline 61 & 6 & 0 & -3.473736 & -0.112679 & 1.857377 \\
\hline 62 & 6 & 0 & -2.677554 & -2.564934 & 2.906120 \\
\hline 63 & 1 & 0 & -2.001635 & -3.029114 & 0.908477 \\
\hline 64 & 6 & 0 & -3.665096 & -0.384488 & 3.205712 \\
\hline 65 & 1 & 0 & -3.822707 & 0.826288 & 1.427560 \\
\hline 66 & 6 & 0 & -3.259553 & -1.607250 & 3.735244 \\
\hline 67 & 1 & 0 & -2.370344 & -3.527128 & 3.313010 \\
\hline 68 & 1 & 0 & -4.145215 & 0.355135 & 3.844297 \\
\hline 69 & 1 & 0 & -3.411670 & -1.820051 & 4.792300 \\
\hline Zero-po & ection= & & & $0.541817\left(\mathrm{H}_{\mathrm{c}}\right.$ & e/Particle) \\
\hline Thermal c & ection to $\mathrm{E}$ & & & .578871 & \\
\hline Thermal c & rrection to $\mathrm{E}$ & alpy= & & 579815 & \\
\hline Thermal c & rrection to $\mathrm{C}$ & bs Free Energy= & & 69339 & \\
\hline Sum of el & tronic and $\mathrm{z}$ & o-point Energies= & & 1904.777800 & \\
\hline Sum of el & tronic and $\mathrm{t}$ & rmal Energies= & & -1904.740746 & \\
\hline Sum of el & tronic and $\mathrm{t}$ & nal Enthalpies= & & -1904.739802 & \\
\hline Sum of el & ronic and $\mathrm{t}$ & nal Free Energic & & 1904.85027 & \\
\hline
\end{tabular}

M06 /6-311++G(d,p)-SDD/SMD//M06/6-31G(d)-LANL2DZ energy in toluene solvent = -1906.9587597

TS2ca

\begin{tabular}{cccccc}
\hline $\begin{array}{c}\text { Center } \\
\text { Number }\end{array}$ & $\begin{array}{c}\text { Atomic } \\
\text { Number }\end{array}$ & $\begin{array}{c}\text { Atomic } \\
\text { Type }\end{array}$ & \multicolumn{2}{c}{ Coordinates (Angstroms) } & X \\
- \\
-1 & 6 & 0 & 0.083287 & 4.165214 & 2.425883 \\
2 & 6 & 0 & -0.559372 & 2.987159 & 2.789040 \\
3 & 6 & 0 & -0.829980 & 2.007001 & 1.835220
\end{tabular}




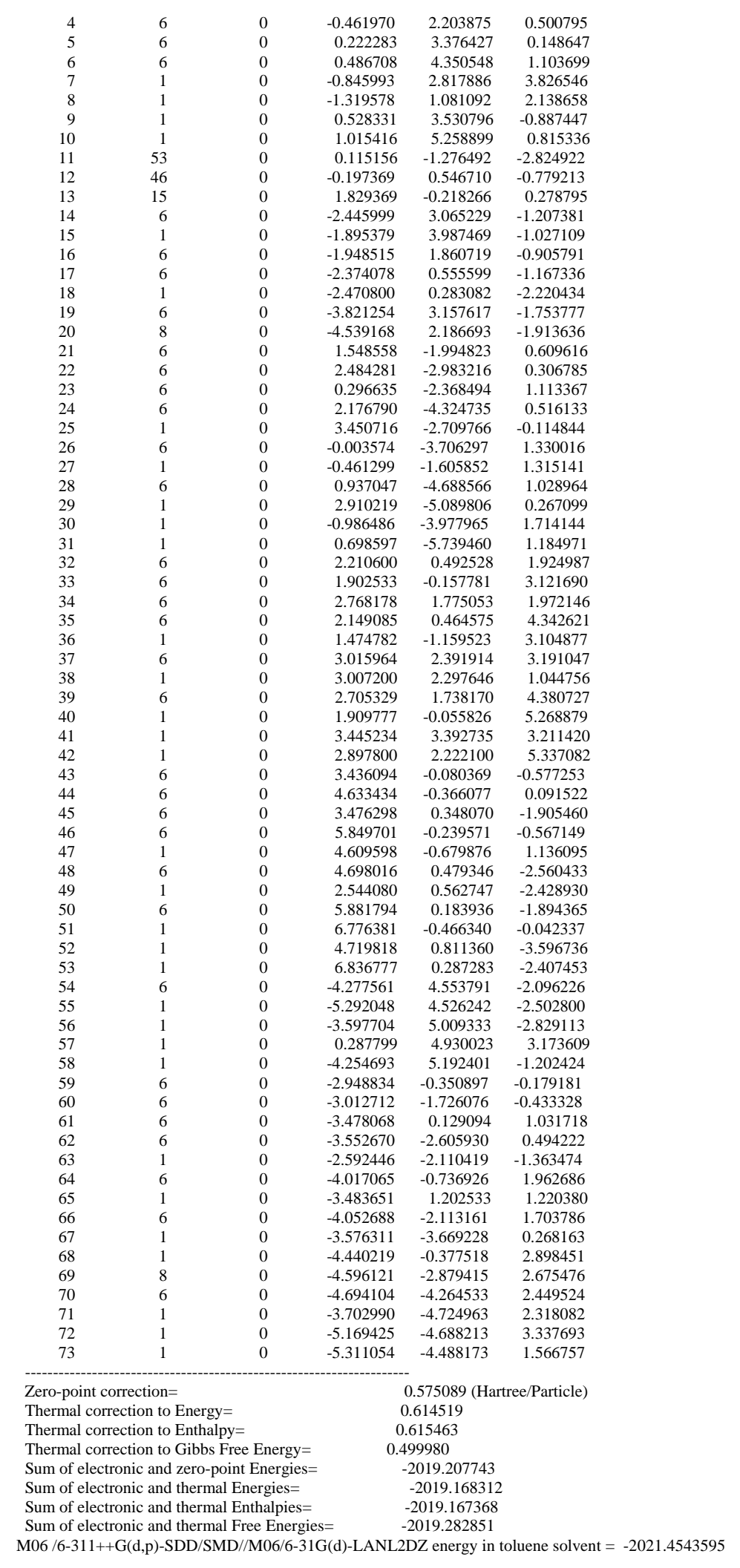

\section{TS2d}

\begin{tabular}{|c|c|c|c|c|c|}
\hline \multirow{2}{*}{$\begin{array}{l}\text { Center } \\
\text { Number }\end{array}$} & \multirow{2}{*}{$\begin{array}{l}\text { Atomic } \\
\text { Number }\end{array}$} & \multirow{2}{*}{$\begin{array}{l}\text { Atomic } \\
\text { Type }\end{array}$} & \multicolumn{3}{|c|}{ Coordinates (Angstroms) } \\
\hline & & & $X$ & $\mathrm{Y}$ & Z \\
\hline 1 & 6 & 0 & -0.486356 & 3.383591 & 2.770382 \\
\hline 2 & 6 & 0 & -1.202615 & 2.193065 & 2.760026 \\
\hline 3 & 6 & 0 & -1.173377 & 1.365825 & 1.637421 \\
\hline 4 & 6 & 0 & -0.409989 & 1.719598 & 0.521656 \\
\hline 5 & 6 & 0 & 0.320970 & 2.909865 & 0.543795 \\
\hline 6 & 6 & 0 & 0.277345 & 3.737894 & 1.659729 \\
\hline 7 & 1 & 0 & -1.789902 & 1.896729 & 3.628238 \\
\hline
\end{tabular}




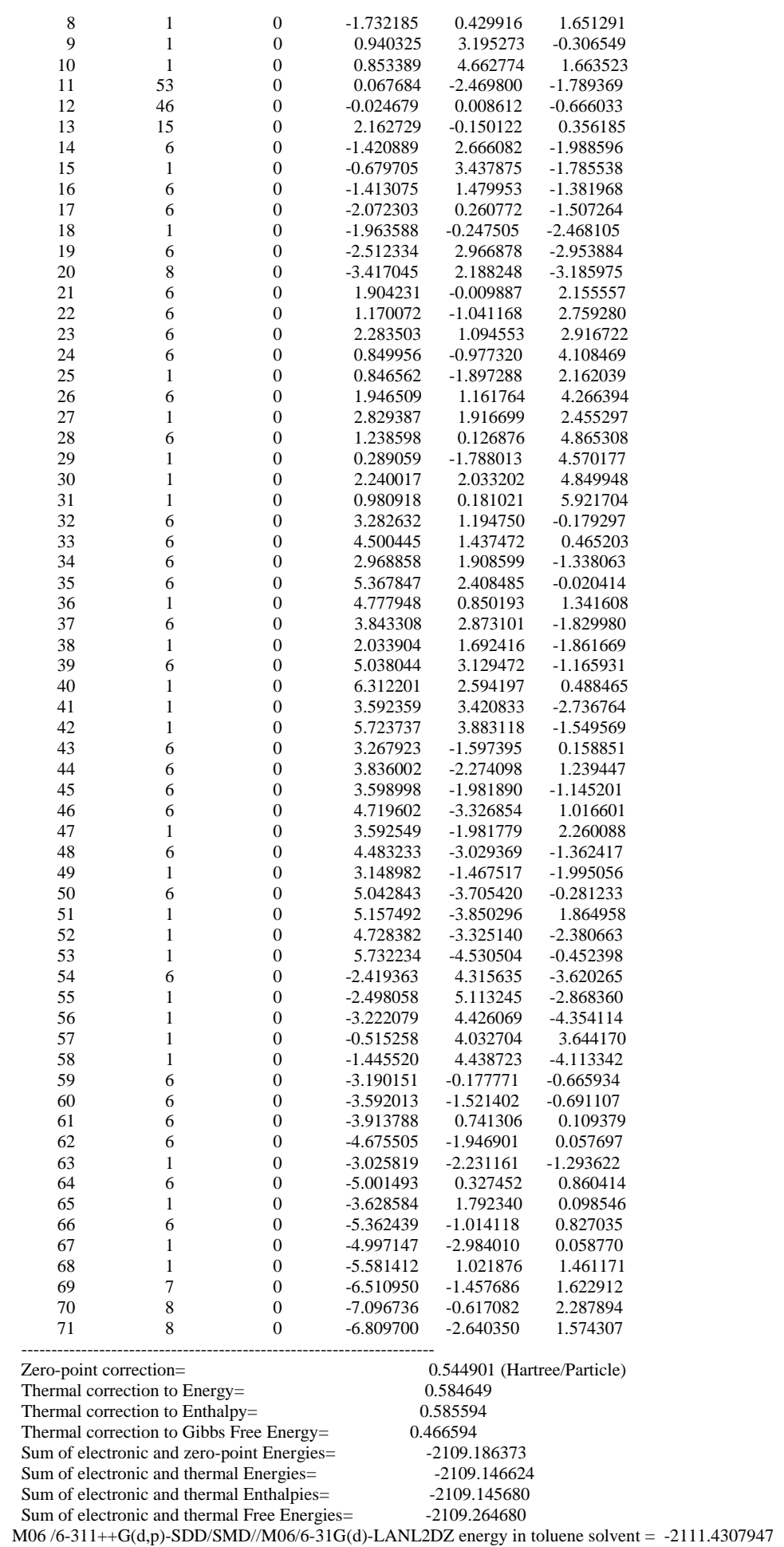

\section{TS2ea}

\begin{tabular}{|c|c|c|c|c|c|}
\hline \multirow{2}{*}{$\begin{array}{l}\text { Center } \\
\text { Number }\end{array}$} & \multirow{2}{*}{$\begin{array}{l}\text { Atomic } \\
\text { Number }\end{array}$} & \multirow{2}{*}{$\begin{array}{l}\text { Atomic } \\
\text { Type }\end{array}$} & \multicolumn{3}{|c|}{ Coordinates (Angstroms) } \\
\hline & & & $\mathrm{X}$ & $\mathrm{Y}$ & Z \\
\hline 1 & 6 & 0 & 1.815540 & 4.177461 & -1.086890 \\
\hline 2 & 6 & 0 & 1.983986 & 3.264149 & -2.120871 \\
\hline 3 & 6 & 0 & 1.888783 & 1.895097 & -1.873013 \\
\hline 4 & 6 & 0 & 1.615064 & 1.430268 & -0.584665 \\
\hline 5 & 6 & 0 & 1.444811 & 2.354947 & 0.451570 \\
\hline 6 & 6 & 0 & 1.545073 & 3.717256 & 0.201000 \\
\hline 7 & 1 & 0 & 2.194412 & 3.613235 & -3.131322 \\
\hline 8 & 1 & 0 & 2.012427 & 1.193873 & -2.697554 \\
\hline 9 & 1 & 0 & 1.214135 & 2.009903 & 1.461745 \\
\hline 10 & 1 & 0 & 1.389564 & 4.427258 & 1.012734 \\
\hline 11 & 53 & 0 & 0.019952 & -3.116438 & -0.613523 \\
\hline 12 & 46 & 0 & 0.848829 & -0.526526 & -0.443950 \\
\hline 13 & 15 & 0 & -1.378542 & 0.297198 & 0.145329 \\
\hline
\end{tabular}




\begin{tabular}{|c|c|c|c|c|c|}
\hline 14 & 6 & 0 & 3.761033 & 0.373068 & 0.861105 \\
\hline 15 & 1 & 0 & 3.500731 & 1.256381 & 1.442963 \\
\hline 16 & 6 & 0 & 2.939636 & -0.160009 & -0.045709 \\
\hline 17 & 6 & 0 & 2.872000 & -1.315110 & -0.809939 \\
\hline 18 & 1 & 0 & 2.899275 & -2.245090 & -0.235952 \\
\hline 19 & 6 & 0 & 5.101826 & -0.234413 & 1.044982 \\
\hline 20 & 8 & 0 & 5.500096 & -1.173468 & 0.379832 \\
\hline 21 & 6 & 0 & -1.667735 & 2.063819 & -0.254051 \\
\hline 22 & 6 & 0 & -1.424687 & 2.469294 & -1.571319 \\
\hline 23 & 6 & 0 & -2.114526 & 3.006503 & 0.674190 \\
\hline 24 & 6 & 0 & -1.646561 & 3.785408 & -1.957342 \\
\hline 25 & 1 & 0 & -1.052001 & 1.746241 & -2.298992 \\
\hline 26 & 6 & 0 & -2.327833 & 4.326411 & 0.287803 \\
\hline 27 & 1 & 0 & -2.291590 & 2.714856 & 1.708624 \\
\hline 28 & 6 & 0 & -2.099398 & 4.716929 & -1.027136 \\
\hline 29 & 1 & 0 & -1.451821 & 4.086532 & -2.985600 \\
\hline 30 & 1 & 0 & -2.678177 & 5.051975 & 1.020402 \\
\hline 31 & 1 & 0 & -2.268878 & 5.749764 & -1.327293 \\
\hline 32 & 6 & 0 & -1.521993 & 0.179954 & 1.967743 \\
\hline 33 & 6 & 0 & -2.745299 & 0.375669 & 2.621135 \\
\hline 34 & 6 & 0 & -0.387735 & -0.122102 & 2.725936 \\
\hline 35 & 6 & 0 & -2.823966 & 0.289385 & 4.005650 \\
\hline 36 & 1 & 0 & -3.642066 & 0.586870 & 2.037565 \\
\hline 37 & 6 & 0 & -0.468587 & -0.211778 & 4.112551 \\
\hline 38 & 1 & 0 & 0.563131 & -0.310849 & 2.221029 \\
\hline 39 & 6 & 0 & -1.685062 & -0.003729 & 4.752447 \\
\hline 40 & 1 & 0 & -3.780214 & 0.442059 & 4.503361 \\
\hline 41 & 1 & 0 & 0.419728 & -0.458806 & 4.691144 \\
\hline 42 & 1 & 0 & -1.750981 & -0.079968 & 5.836531 \\
\hline 43 & 6 & 0 & -2.916421 & -0.517217 & -0.436844 \\
\hline 44 & 6 & 0 & -3.717415 & 0.037609 & -1.438229 \\
\hline 45 & 6 & 0 & -3.275187 & -1.754873 & 0.111811 \\
\hline 46 & 6 & 0 & -4.853304 & -0.631774 & -1.883041 \\
\hline 47 & 1 & 0 & -3.468520 & 1.005669 & -1.869764 \\
\hline 48 & 6 & 0 & -4.412515 & -2.416873 & -0.332133 \\
\hline 49 & 1 & 0 & -2.663676 & -2.205204 & 0.891640 \\
\hline 50 & 6 & 0 & -5.203205 & -1.859070 & -1.332366 \\
\hline 51 & 1 & 0 & -5.469437 & -0.184099 & -2.661144 \\
\hline 52 & 1 & 0 & -4.677294 & -3.378257 & 0.104186 \\
\hline 53 & 1 & 0 & -6.092819 & -2.381317 & -1.680671 \\
\hline 54 & 6 & 0 & 5.945861 & 0.397099 & 2.123302 \\
\hline 55 & 1 & 0 & 5.423187 & 0.366711 & 3.089116 \\
\hline 56 & 1 & 0 & 6.125791 & 1.456451 & 1.893557 \\
\hline 57 & 1 & 0 & 1.891749 & 5.246135 & -1.280686 \\
\hline 58 & 1 & 0 & 6.901462 & -0.127639 & 2.205410 \\
\hline 59 & 6 & 0 & 3.248090 & -1.436779 & -2.252326 \\
\hline 60 & 1 & 0 & 3.164759 & -0.489872 & -2.794627 \\
\hline 61 & 1 & 0 & 4.300937 & -1.753703 & -2.295531 \\
\hline 62 & 1 & 0 & 2.638319 & -2.197515 & -2.753136 \\
\hline Cero-poi & ion $=$ & \multicolumn{4}{|c|}{0.489034 (Hartree/Particle) } \\
\hline Thermal & a to & \multicolumn{4}{|c|}{0.522286} \\
\hline Thermal & a to & \multicolumn{4}{|c|}{0.523230} \\
\hline Thermal & 1 to & \multicolumn{4}{|c|}{0.421294} \\
\hline Sum of $\mathrm{e}$ & and & \multicolumn{4}{|c|}{-1713.239174} \\
\hline sum of $\mathrm{e}$ & and & \multicolumn{4}{|c|}{-1713.205923} \\
\hline um of $\mathrm{e}$ & and & \multicolumn{4}{|c|}{-1713.204978} \\
\hline Sum of $\mathrm{e}$ & and & \multicolumn{4}{|c|}{-1713.306914} \\
\hline
\end{tabular}

TS2fa

\begin{tabular}{rrrrrr} 
Center & $\begin{array}{c}\text { Atomic } \\
\text { Number }\end{array}$ & Number & $\begin{array}{c}\text { Atomic } \\
\text { Type }\end{array}$ & \multicolumn{2}{c}{ Coordinates (Angstroms) } \\
-1 & 6 & 0 & 0.820215 & 4.520006 & -0.698639 \\
\hline 1 & 6 & 0 & 1.074641 & 3.642938 & -1.746341 \\
3 & 6 & 0 & 1.252401 & 2.282213 & -1.497869 \\
4 & 6 & 0 & 1.165101 & 1.784419 & -0.196046 \\
5 & 6 & 0 & 0.874945 & 2.670043 & 0.851302 \\
6 & 6 & 0 & 0.713318 & 4.026756 & 0.601032 \\
7 & 1 & 0 & 1.142291 & 4.015342 & -2.767753 \\
8 & 1 & 0 & 1.459039 & 1.612014 & -2.332155 \\
9 & 1 & 0 & 0.777097 & 2.288060 & 1.869745 \\
10 & 1 & 0 & 0.484850 & 4.702973 & 1.424989 \\
11 & 53 & 0 & 0.297884 & -2.916668 & 0.626159 \\
12 & 46 & 0 & 0.708588 & -0.263513 & 0.072786 \\
13 & 15 & 0 & -1.681091 & 0.149688 & 0.042451 \\
14 & 6 & 0 & 3.493032 & 1.263714 & 1.171682 \\
15 & 1 & 0 & 3.140373 & 2.188593 & 1.624729 \\
16 & 6 & 0 & 2.726714 & 0.516752 & 0.367827 \\
17 & 6 & 0 & 2.841462 & -0.740913 & -0.211745 \\
18 & 1 & 0 & 3.005796 & -1.543001 & 0.513578 \\
19 & 6 & 0 & 4.881169 & 0.832659 & 1.454761 \\
20 & 8 & 0 & 5.398321 & -0.148471 & 0.947353 \\
21 & 6 & 0 & -2.606784 & -1.143879 & -0.862169 \\
22 & 6 & 0 & -3.796599 & -1.704606 & -0.398907 \\
23 & 6 & 0 & -2.063841 & -1.595700 & -2.069794 \\
24 & 6 & 0 & -4.438862 & -2.691959 & -1.140962 \\
25 & 1 & 0 & -4.218590 & -1.387013 & 0.553369 \\
26 & 6 & 0 & -2.714293 & -2.568159 & -2.816991 \\
27 & 1 & 0 & -1.106645 & -1.198516 & -2.412382
\end{tabular}




\begin{tabular}{|c|c|c|c|c|c|}
\hline 28 & 6 & 0 & -3.904379 & -3.120136 & -2.350493 \\
\hline 29 & 1 & 0 & -5.361438 & -3.131355 & -0.765109 \\
\hline 30 & 1 & 0 & -2.279978 & -2.912963 & -3.753668 \\
\hline 31 & 1 & 0 & -4.408264 & -3.895485 & -2.925111 \\
\hline 32 & 6 & 0 & -2.243791 & 1.704659 & -0.759380 \\
\hline 33 & 6 & 0 & -2.484204 & 1.742859 & -2.136922 \\
\hline 34 & 6 & 0 & -2.337459 & 2.892765 & -0.027743 \\
\hline 35 & 6 & 0 & -2.820384 & 2.936326 & -2.764761 \\
\hline 36 & 1 & 0 & -2.427217 & 0.829085 & -2.727079 \\
\hline 37 & 6 & 0 & -2.678418 & 4.084987 & -0.657053 \\
\hline 38 & 1 & 0 & -2.142436 & 2.889389 & 1.044234 \\
\hline 39 & 6 & 0 & -2.918760 & 4.111282 & -2.026294 \\
\hline 40 & 1 & 0 & -3.014017 & 2.944739 & -3.836208 \\
\hline 41 & 1 & 0 & -2.746403 & 5.000720 & -0.070914 \\
\hline 42 & 1 & 0 & -3.185872 & 5.045744 & -2.517343 \\
\hline 43 & 6 & 0 & -2.402048 & 0.240135 & 1.717867 \\
\hline 44 & 6 & 0 & -3.701815 & 0.712908 & 1.943773 \\
\hline 45 & 6 & 0 & -1.625151 & -0.170043 & 2.804098 \\
\hline 46 & 6 & 0 & -4.214515 & 0.761711 & 3.234107 \\
\hline 47 & 1 & 0 & -4.307836 & 1.054333 & 1.103656 \\
\hline 48 & 6 & 0 & -2.140943 & -0.118176 & 4.095784 \\
\hline 49 & 1 & 0 & -0.616028 & -0.546618 & 2.629058 \\
\hline 50 & 6 & 0 & -3.433781 & 0.345759 & 4.310627 \\
\hline 51 & 1 & 0 & -5.225610 & 1.129396 & 3.401302 \\
\hline 52 & 1 & 0 & -1.528845 & -0.443698 & 4.934851 \\
\hline 53 & 1 & 0 & -3.837211 & 0.387233 & 5.321175 \\
\hline 54 & 6 & 0 & 5.626504 & 1.701644 & 2.436660 \\
\hline 55 & 1 & 0 & 5.084157 & 1.758400 & 3.390222 \\
\hline 56 & 1 & 0 & 5.702562 & 2.728407 & 2.052874 \\
\hline 57 & 1 & 0 & 0.686927 & 5.582999 & -0.893262 \\
\hline 58 & 1 & 0 & 6.628095 & 1.297671 & 2.606201 \\
\hline 59 & 6 & 0 & 3.298508 & -1.047921 & -1.640938 \\
\hline 60 & 6 & 0 & 4.153429 & -2.315213 & -1.557653 \\
\hline 61 & 1 & 0 & 5.003998 & -2.164183 & -0.881001 \\
\hline 62 & 1 & 0 & 3.560577 & -3.164385 & -1.189529 \\
\hline 63 & 1 & 0 & 4.541928 & -2.574774 & -2.552684 \\
\hline 64 & 6 & 0 & 2.136624 & -1.307977 & -2.605010 \\
\hline 65 & 1 & 0 & 1.477851 & -0.432508 & -2.704430 \\
\hline 66 & 1 & 0 & 2.540182 & -1.535236 & -3.603237 \\
\hline 67 & 1 & 0 & 1.524565 & -2.158118 & -2.275725 \\
\hline 68 & 6 & 0 & 4.159009 & 0.102311 & -2.170571 \\
\hline 69 & 1 & 0 & 4.529798 & -0.144705 & -3.175621 \\
\hline 70 & 1 & 0 & 3.595128 & 1.042329 & -2.242608 \\
\hline 71 & 1 & 0 & 5.021825 & 0.269577 & -1.513782 \\
\hline \multicolumn{3}{|c|}{ Zero-point correction $=$} & \multicolumn{3}{|c|}{0.573964 (Hartree/Particle) } \\
\hline \multicolumn{3}{|c|}{ Thermal correction to Energy= } & \multicolumn{2}{|c|}{0.611557} & \\
\hline \multicolumn{3}{|c|}{ Thermal correction to Enthalpy= } & \multicolumn{2}{|c|}{0.612502} & \\
\hline \multicolumn{3}{|c|}{ Thermal correction to Gibbs Free Energy= } & \multicolumn{2}{|c|}{0.501950} & \\
\hline \multicolumn{3}{|c|}{ Sum of electronic and zero-point Energies $=$} & \multicolumn{2}{|c|}{-1830.998357} & \\
\hline \multicolumn{3}{|c|}{ Sum of electronic and thermal Energies= } & \multicolumn{2}{|c|}{-1830.960763} & \\
\hline \multirow{2}{*}{\multicolumn{3}{|c|}{ Sum of electronic and thermal Enthalpies $=$}} & \multicolumn{2}{|c|}{-1830.959819} & \\
\hline & & & \multicolumn{2}{|c|}{-1831.070371} & \\
\hline
\end{tabular}

TS2ga

\begin{tabular}{|c|c|c|c|c|c|}
\hline \multirow{2}{*}{$\begin{array}{l}\text { Center } \\
\text { Number }\end{array}$} & \multirow{2}{*}{$\begin{array}{l}\text { Atomic } \\
\text { Number }\end{array}$} & \multirow{2}{*}{$\begin{array}{l}\text { Atomic } \\
\text { Type }\end{array}$} & \multicolumn{3}{|c|}{ Coordinates (Angstroms) } \\
\hline & & & $\mathrm{X}$ & $\mathrm{Y}$ & \\
\hline 1 & 6 & 0 & -1.291516 & 4.315240 & 1.164432 \\
\hline 2 & 6 & 0 & -1.595248 & 3.255528 & 2.011263 \\
\hline 3 & 6 & 0 & -1.578061 & 1.944618 & 1.535144 \\
\hline 4 & 6 & 0 & -1.228653 & 1.693929 & 0.207411 \\
\hline 5 & 6 & 0 & -0.917302 & 2.758020 & -0.641064 \\
\hline 6 & 6 & 0 & -0.956447 & 4.063490 & -0.164988 \\
\hline 7 & 1 & 0 & -1.864326 & 3.444438 & 3.049784 \\
\hline 8 & 1 & 0 & -1.850104 & 1.126334 & 2.199828 \\
\hline 9 & 1 & 0 & -0.646221 & 2.565033 & -1.680648 \\
\hline 10 & 1 & 0 & -0.699517 & 4.887290 & -0.830381 \\
\hline 11 & 53 & 0 & -0.064562 & -2.954862 & -0.422435 \\
\hline 12 & 46 & 0 & -0.684223 & -0.297905 & -0.248111 \\
\hline 13 & 15 & 0 & 1.662118 & 0.220213 & -0.068297 \\
\hline 14 & 6 & 0 & -3.431988 & 1.150052 & -1.473493 \\
\hline 15 & 1 & 0 & -3.079912 & 2.106568 & -1.855188 \\
\hline 16 & 6 & 0 & -2.691466 & 0.363041 & -0.701125 \\
\hline 17 & 6 & 0 & -2.748678 & -0.911414 & -0.161518 \\
\hline 18 & 1 & 0 & -2.884773 & -1.754419 & -0.839028 \\
\hline 19 & 6 & 0 & -4.829822 & 0.721190 & -1.767206 \\
\hline 20 & 8 & 0 & -5.304038 & -0.312486 & -1.339413 \\
\hline 21 & 6 & 0 & 2.225229 & -0.604286 & 1.459616 \\
\hline 22 & 6 & 0 & 3.316379 & -1.471537 & 1.503396 \\
\hline 23 & 6 & 0 & 1.448466 & -0.417755 & 2.610021 \\
\hline 24 & 6 & 0 & 3.638743 & -2.126339 & 2.688260 \\
\hline 25 & 1 & 0 & 3.901075 & -1.661297 & 0.604688 \\
\hline 26 & 6 & 0 & 1.781295 & -1.062975 & 3.793743 \\
\hline 27 & 1 & 0 & 0.565841 & 0.224232 & 2.570887 \\
\hline 28 & 6 & 0 & 2.878134 & -1.919487 & 3.833433 \\
\hline 29 & 1 & 0 & 4.485557 & -2.809953 & 2.710817 \\
\hline 30 & 1 & 0 & 1.171106 & -0.911660 & 4.682182 \\
\hline 31 & 1 & 0 & 3.130594 & -2.438637 & 4.756386 \\
\hline 32 & 6 & 0 & 2.148682 & 1.979865 & 0.124891 \\
\hline 33 & 6 & 0 & 2.333051 & 2.578818 & 1.371781 \\
\hline
\end{tabular}




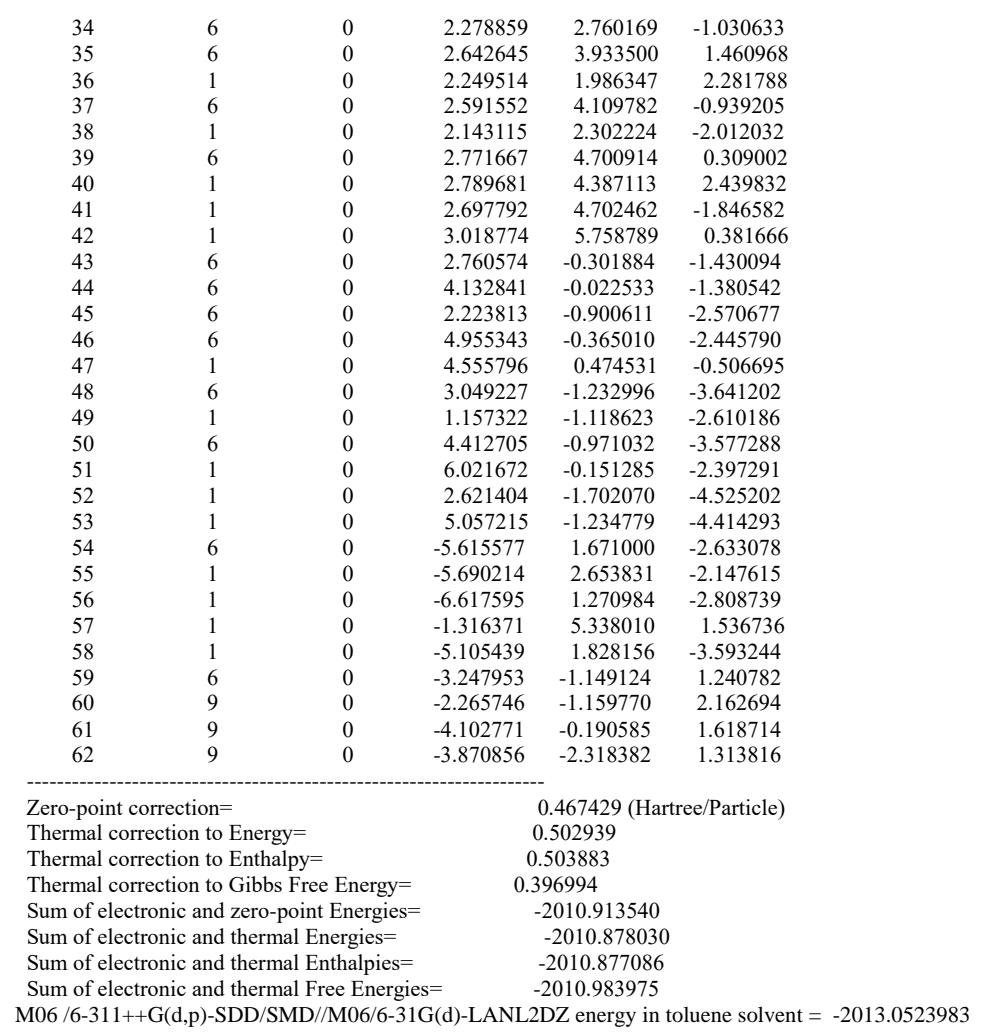

TS2ha

\begin{tabular}{rrrrrr} 
Center & Atomic & Atomic & \multicolumn{2}{c}{ Coordinates (Angstroms) } \\
Number & Number & Type & $\mathrm{X}$ & $\mathrm{Y}$ & $\mathrm{Z}$ \\
---------------- & & \\
1 & 6 & 0 & 2.094417 & 3.862558 & -1.776746 \\
2 & 6 & 0 & 2.040931 & 2.776610 & -2.641818 \\
3 & 6 & 0 & 1.876990 & 1.486393 & -2.140288 \\
4 & 6 & 0 & 1.740353 & 1.264559 & -0.763610 \\
5 & 6 & 0 & 1.783091 & 2.368615 & 0.095576 \\
6 & 6 & 0 & 1.966604 & 3.651429 & -0.406163 \\
7 & 1 & 0 & 2.133481 & 2.924986 & -3.716981 \\
8 & 1 & 0 & 1.839717 & 0.655532 & -2.844605 \\
9 & 1 & 0 & 1.644129 & 2.243003 & 1.166182 \\
10 & 1 & 0 & 1.993454 & 4.495381 & 0.282272 \\
11 & 53 & 0 & -0.278038 & -3.090036 & -0.596712 \\
12 & 46 & 0 & 0.799483 & -0.592530 & -0.379806 \\
13 & 15 & 0 & -1.326131 & 0.344411 & 0.255284 \\
14 & 6 & 0 & 3.710940 & -0.227285 & 0.890216 \\
15 & 6 & 0 & 2.908710 & -0.425580 & -0.168345 \\
16 & 6 & 0 & 2.711928 & -1.351581 & -1.168406 \\
17 & 1 & 0 & 2.859672 & -2.405182 & -0.935312 \\
18 & 6 & 0 & 5.091092 & -0.783367 & 0.743191 \\
19 & 8 & 0 & 5.445658 & -1.412497 & -0.237216 \\
20 & 6 & 0 & -2.536485 & 0.019152 & -1.075779 \\
21 & 6 & 0 & -3.846279 & -0.399018 & -0.840460 \\
22 & 6 & 0 & -2.088666 & 0.157031 & -2.394794 \\
23 & 6 & 0 & -4.702413 & -0.648650 & -1.908833 \\
24 & 1 & 0 & -4.198804 & -0.553148 & 0.178106 \\
25 & 6 & 0 & -2.948479 & -0.082105 & -3.458730 \\
26 & 1 & 0 & -1.050404 & 0.437308 & -2.585477 \\
27 & 6 & 0 & -4.258936 & -0.484757 & -3.216039 \\
28 & 1 & 0 & -5.720251 & -0.982865 & -1.715104 \\
29 & 1 & 0 & -2.588507 & 0.027538 & -4.480116 \\
30 & 1 & 0 & -4.930352 & -0.686279 & -4.048937 \\
31 & 6 & 0 & -1.367432 & 2.166643 & 0.473432 \\
32 & 6 & 0 & -1.626848 & 3.026913 & -0.594992 \\
33 & 6 & 0 & -1.016546 & 2.711447 & 1.714305 \\
34 & 6 & 0 & -1.548158 & 4.405266 & -0.422833 \\
35 & 1 & 0 & -1.900863 & 2.621417 & -1.568672 \\
36 & 6 & 0 & -0.948076 & 4.088764 & 1.886882 \\
37 & 1 & 0 & -0.799031 & 2.051962 & 2.556187 \\
38 & 6 & 0 & -1.214230 & 4.939388 & 0.817241 \\
39 & 1 & 0 & -1.756490 & 5.064510 & -1.263914 \\
40 & 1 & 0 & -0.686751 & 4.499175 & 2.861181 \\
41 & 1 & 0 & -1.161525 & 6.018687 & 0.951348 \\
42 & 6 & 0 & -2.065763 & -0.241225 & 1.819922 \\
43 & 6 & 0 & -3.216078 & 0.368174 & 2.340441 \\
44 & 6 & 0 & -1.457757 & -1.275466 & 2.533836 \\
45 & 6 & 0 & -3.758043 & -0.068689 & 3.542072 \\
46 & 1 & 0 & -3.682646 & 1.196839 & 1.806381 \\
47 & 6 & 0 & -1.999754 & -1.706117 & 3.742095 \\
48 & 1 & 0 & -0.567745 & -1.755843 & 2.127589
\end{tabular}




$\begin{array}{rrrrrr}49 & 6 & 0 & -3.149721 & -1.107993 & 4.243454 \\ 50 & 1 & 0 & -4.655342 & 0.405685 & 3.936002 \\ 51 & 1 & 0 & -1.519295 & -2.516195 & 4.287529 \\ 52 & 1 & 0 & -3.573819 & -1.448498 & 5.186789 \\ 53 & 6 & 0 & 6.050095 & -0.541973 & 1.886368 \\ 54 & 1 & 0 & 5.754288 & -1.123565 & 2.770495 \\ 55 & 1 & 0 & 6.071108 & 0.513509 & 2.187559 \\ 56 & 1 & 0 & 2.233094 & 4.869173 & -2.167697 \\ 57 & 1 & 0 & 2.689618 & -1.091764 & -2.225296 \\ 58 & 1 & 0 & 7.049463 & -0.859435 & 1.576863 \\ 59 & 6 & 0 & 3.308917 & 0.480798 & 2.144864 \\ 60 & 1 & 0 & 3.695398 & -0.043176 & 3.028457 \\ 61 & 1 & 0 & 3.691174 & 1.512834 & 2.190914 \\ 62 & 1 & 0 & 2.217263 & 0.510914 & 2.247191 \\ ------------------------------------------------- & & \end{array}$

$\begin{array}{lc}\text { Zero-point correction= } & 0.489019 \text { (Hartree/Particle) } \\ \text { Thermal correction to Energy= } & 0.522745 \\ \text { Thermal correction to Enthalpy= } & 0.523689 \\ \text { Thermal correction to Gibbs Free Energy }= & 0.420911 \\ \text { Sum of electronic and zero-point Energies }= & -1713.237422 \\ \text { Sum of electronic and thermal Energies }= & -1713.203696 \\ \text { Sum of electronic and thermal Enthalpies }= & -1713.202752 \\ \text { Sum of electronic and thermal Free Energies }= & -1713.305530 \\ \text { M06 /6-311++G(d,p)-SDD/SMD/M06/6-31G(d)-LANL2DZ energy in toluene solvent }=-1715.3153042\end{array}$

M06 /6-311++G(d,p)-SDD/SMD//M06/6-31G(d)-LANL2DZ energy in toluene solvent $=-1715.3153042$

TS2ia

\begin{tabular}{|c|c|c|c|c|c|}
\hline \multirow{2}{*}{$\begin{array}{l}\text { Center } \\
\text { Number }\end{array}$} & \multirow{2}{*}{$\begin{array}{l}\text { Atomic } \\
\text { Number }\end{array}$} & \multirow{2}{*}{$\begin{array}{l}\text { Atomic } \\
\text { Type }\end{array}$} & \multicolumn{3}{|c|}{ Coordinates (Angstroms) } \\
\hline & & & $\mathrm{X}$ & $\mathrm{Y}$ & \\
\hline 1 & 6 & 0 & 1.842678 & 2.368168 & -3.633055 \\
\hline 2 & 6 & 0 & 1.356417 & 1.133287 & -4.044443 \\
\hline 3 & 6 & 0 & 1.106334 & 0.131209 & -3.107543 \\
\hline 4 & 6 & 0 & 1.316604 & 0.366853 & -1.744237 \\
\hline 5 & 6 & 0 & 1.793559 & 1.614402 & -1.337680 \\
\hline 6 & 6 & 0 & 2.063186 & 2.602323 & -2.277714 \\
\hline 7 & 1 & 0 & 1.174700 & 0.936850 & -5.100396 \\
\hline 8 & 1 & 0 & 0.727790 & -0.829768 & -3.456804 \\
\hline 9 & 1 & 0 & 1.943327 & 1.832405 & -0.283516 \\
\hline 10 & 1 & 0 & 2.432026 & 3.570594 & -1.940039 \\
\hline 11 & 53 & 0 & -1.257402 & -3.172452 & 0.214984 \\
\hline 12 & 46 & 0 & 0.237098 & -1.024355 & -0.575051 \\
\hline 13 & 15 & 0 & -1.418722 & 0.565651 & 0.187896 \\
\hline 14 & 6 & 0 & 3.365103 & -0.976159 & 0.003593 \\
\hline 15 & 6 & 0 & 2.328771 & -1.310834 & -0.788132 \\
\hline 16 & 6 & 0 & 1.757311 & -2.406198 & -1.390953 \\
\hline 17 & 1 & 0 & 1.760888 & -3.357936 & -0.859902 \\
\hline 18 & 6 & 0 & 4.563899 & -1.870323 & -0.106378 \\
\hline 19 & 8 & 0 & 4.647999 & -2.735467 & -0.957493 \\
\hline 20 & 6 & 0 & -3.051022 & 0.224870 & -0.562653 \\
\hline 21 & 6 & 0 & -4.256568 & 0.476555 & 0.093103 \\
\hline 22 & 6 & 0 & -3.070158 & -0.290904 & -1.862548 \\
\hline 23 & 6 & 0 & -5.465028 & 0.227098 & -0.549571 \\
\hline 24 & 1 & 0 & -4.256777 & 0.852317 & 1.115524 \\
\hline 25 & 6 & 0 & -4.278342 & -0.527070 & -2.506692 \\
\hline 26 & 1 & 0 & -2.127452 & -0.522070 & -2.363745 \\
\hline 27 & 6 & 0 & -5.477541 & -0.269288 & -1.848714 \\
\hline 28 & 1 & 0 & -6.402024 & 0.416669 & -0.028509 \\
\hline 29 & 1 & 0 & -4.283107 & -0.930163 & -3.517672 \\
\hline 30 & 1 & 0 & -6.425451 & -0.466089 & -2.346878 \\
\hline 31 & 6 & 0 & -1.099318 & 2.315070 & -0.265113 \\
\hline 32 & 6 & 0 & -1.536405 & 2.823028 & -1.490692 \\
\hline 33 & 6 & 0 & -0.294085 & 3.117068 & 0.551454 \\
\hline 34 & 6 & 0 & -1.184593 & 4.109537 & -1.884489 \\
\hline 35 & 1 & 0 & -2.158789 & 2.211449 & -2.143793 \\
\hline 36 & 6 & 0 & 0.050068 & 4.405149 & 0.160018 \\
\hline 37 & 1 & 0 & 0.078713 & 2.729169 & 1.499605 \\
\hline 38 & 6 & 0 & -0.396044 & 4.905438 & -1.059881 \\
\hline 39 & 1 & 0 & -1.534218 & 4.492313 & -2.842000 \\
\hline 40 & 1 & 0 & 0.676189 & 5.015177 & 0.810121 \\
\hline 41 & 1 & 0 & -0.126716 & 5.914236 & -1.369100 \\
\hline 42 & 6 & 0 & -1.677642 & 0.651201 & 1.999845 \\
\hline 43 & 6 & 0 & -2.308289 & 1.752753 & 2.594565 \\
\hline 44 & 6 & 0 & -1.217045 & -0.386550 & 2.814603 \\
\hline 45 & 6 & 0 & -2.467276 & 1.813330 & 3.973997 \\
\hline 46 & 1 & 0 & -2.659254 & 2.578723 & 1.975182 \\
\hline 47 & 6 & 0 & -1.377551 & -0.323410 & 4.195911 \\
\hline 48 & 1 & 0 & -0.748906 & -1.259843 & 2.360029 \\
\hline 49 & 6 & 0 & -1.998270 & 0.776464 & 4.777273 \\
\hline 50 & 1 & 0 & -2.956792 & 2.675579 & 4.423900 \\
\hline 51 & 1 & 0 & -1.015865 & -1.141443 & 4.816132 \\
\hline 52 & 1 & 0 & -2.119921 & 0.827838 & 5.858046 \\
\hline 53 & 6 & 0 & 5.654591 & -1.693880 & 0.923650 \\
\hline 54 & 1 & 0 & 5.248654 & -1.536616 & 1.930130 \\
\hline 55 & 1 & 0 & 6.270901 & -0.816227 & 0.687135 \\
\hline 56 & 1 & 0 & 2.047470 & 3.147382 & -4.365566 \\
\hline 57 & 1 & 0 & 1.554395 & -2.449149 & -2.459445 \\
\hline 58 & 1 & 0 & 6.294888 & -2.580455 & 0.904897 \\
\hline 59 & 6 & 0 & 3.304285 & 0.140901 & 0.965422 \\
\hline 60 & 6 & 0 & 4.241137 & 1.181591 & 0.940411 \\
\hline 61 & 6 & 0 & 2.253089 & 0.209677 & 1.888207 \\
\hline 62 & 6 & 0 & 4.113648 & 2.271174 & 1.793525 \\
\hline 63 & 1 & 0 & 5.047764 & 1.155303 & 0.207431 \\
\hline
\end{tabular}




\begin{tabular}{|c|c|c|c|c|c|}
\hline 64 & 6 & 0 & 2.123409 & 1.299360 & 2.743707 \\
\hline 65 & 1 & 0 & 1.530679 & -0.606892 & 1.926410 \\
\hline 66 & 6 & 0 & 3.050596 & 2.336973 & 2.692535 \\
\hline 67 & 1 & 0 & 4.840857 & 3.080308 & 1.748377 \\
\hline 68 & 1 & 0 & 1.289813 & 1.333679 & 3.447444 \\
\hline 69 & 1 & 0 & 2.951819 & 3.192736 & 3.358729 \\
\hline \multicolumn{4}{|c|}{ Zero-point correction $=$} & \multicolumn{2}{|c|}{0.542449 (Hartree/Particle) } \\
\hline \multicolumn{3}{|c|}{ Thermal correction to Energy= } & \multicolumn{2}{|c|}{0.579043} & \\
\hline \multicolumn{3}{|c|}{ Thermal correction to Enthalpy= } & \multicolumn{2}{|c|}{0.579987} & \\
\hline \multirow{2}{*}{\multicolumn{3}{|c|}{$\begin{array}{l}\text { Thermal correction to Gibbs Free Energy= } \\
\text { Sum of electronic and zero-point Energies= }\end{array}$}} & \multicolumn{2}{|c|}{0.471709} & \\
\hline & & & \multicolumn{2}{|r|}{-1904.776687} & \\
\hline \multicolumn{3}{|c|}{ Sum of electronic and thermal Energies= } & \multicolumn{2}{|r|}{-1904.740093} & \\
\hline \multicolumn{3}{|c|}{ Sum of electronic and thermal Enthalpies= } & \multirow{2}{*}{\multicolumn{2}{|c|}{$\begin{array}{l}-1904.739149 \\
-1904.847428\end{array}$}} & \\
\hline \multicolumn{3}{|c|}{ Sum of electronic and thermal Free Energies $=$} & & & \\
\hline
\end{tabular}

TS2ja

\begin{tabular}{|c|c|c|c|c|c|}
\hline \multirow{2}{*}{$\begin{array}{l}\text { Center } \\
\text { Number }\end{array}$} & \multirow{2}{*}{$\begin{array}{l}\text { Atomic } \\
\text { Number }\end{array}$} & \multirow{2}{*}{$\begin{array}{l}\text { Atomic } \\
\text { Type }\end{array}$} & \multicolumn{3}{|c|}{ Coordinates (Angstroms) } \\
\hline & & & $\mathrm{X}$ & $\mathrm{Y}$ & \\
\hline 1 & 6 & 0 & 1.754292 & 3.685327 & -2.305241 \\
\hline 2 & 6 & 0 & 1.468499 & 2.604469 & -3.130158 \\
\hline 3 & 6 & 0 & 1.309187 & 1.331389 & -2.584382 \\
\hline 4 & 6 & 0 & 1.413670 & 1.131963 & -1.202667 \\
\hline 5 & 6 & 0 & 1.700043 & 2.225465 & -0.381618 \\
\hline 6 & 6 & 0 & 1.872242 & 3.489297 & -0.930736 \\
\hline 7 & 1 & 0 & 1.371875 & 2.742761 & -4.206155 \\
\hline 8 & 1 & 0 & 1.077295 & 0.502389 & -3.253375 \\
\hline 9 & 1 & 0 & 1.782184 & 2.103394 & 0.693879 \\
\hline 10 & 1 & 0 & 2.089920 & 4.330324 & -0.273426 \\
\hline 11 & 53 & 0 & -0.785023 & -3.088348 & -0.572983 \\
\hline 12 & 46 & 0 & 0.441347 & -0.666869 & -0.621206 \\
\hline 13 & 15 & 0 & -1.500314 & 0.416906 & 0.281902 \\
\hline 14 & 6 & 0 & 3.520657 & -0.529976 & 0.190302 \\
\hline 15 & 6 & 0 & 2.527690 & -0.632362 & -0.716558 \\
\hline 16 & 6 & 0 & 2.184626 & -1.520514 & -1.709120 \\
\hline 17 & 1 & 0 & 2.338486 & -2.581358 & -1.517378 \\
\hline 18 & 6 & 0 & 4.854622 & -1.064015 & -0.226960 \\
\hline 19 & 8 & 0 & 4.982964 & -1.693198 & -1.260644 \\
\hline 20 & 6 & 0 & -2.920886 & 0.110663 & -0.823914 \\
\hline 21 & 6 & 0 & -4.156001 & -0.350142 & -0.368186 \\
\hline 22 & 6 & 0 & -2.717610 & 0.277042 & -2.199323 \\
\hline 23 & 6 & 0 & -5.180253 & -0.613449 & -1.273004 \\
\hline 24 & 1 & 0 & -4.317564 & -0.525658 & 0.694100 \\
\hline 25 & 6 & 0 & -3.745522 & 0.025681 & -3.098212 \\
\hline 26 & 1 & 0 & -1.739128 & 0.590597 & -2.568765 \\
\hline 27 & 6 & 0 & -4.980429 & -0.420203 & -2.634933 \\
\hline 28 & 1 & 0 & -6.137432 & -0.981623 & -0.907751 \\
\hline 29 & 1 & 0 & -3.576560 & 0.160030 & -4.165142 \\
\hline 30 & 1 & 0 & -5.782635 & -0.630964 & -3.339950 \\
\hline 31 & 6 & 0 & -1.386597 & 2.240933 & 0.434916 \\
\hline 32 & 6 & 0 & -1.774461 & 3.097832 & -0.596287 \\
\hline 33 & 6 & 0 & -0.783880 & 2.780769 & 1.577787 \\
\hline 34 & 6 & 0 & -1.568872 & 4.469443 & -0.484367 \\
\hline 35 & 1 & 0 & -2.245429 & 2.698808 & -1.493814 \\
\hline 36 & 6 & 0 & -0.589407 & 4.151890 & 1.690942 \\
\hline 37 & 1 & 0 & -0.463224 & 2.122761 & 2.386695 \\
\hline 38 & 6 & 0 & -0.981308 & 4.999918 & 0.658895 \\
\hline 39 & 1 & 0 & -1.877234 & 5.126251 & -1.296199 \\
\hline 40 & 1 & 0 & -0.129999 & 4.558923 & 2.590397 \\
\hline 41 & 1 & 0 & -0.828791 & 6.074451 & 0.746888 \\
\hline 42 & 6 & 0 & -1.993671 & -0.078421 & 1.967336 \\
\hline 43 & 6 & 0 & -3.029616 & 0.595147 & 2.629446 \\
\hline 44 & 6 & 0 & -1.297285 & -1.088456 & 2.633123 \\
\hline 45 & 6 & 0 & -3.375092 & 0.243997 & 3.927594 \\
\hline 46 & 1 & 0 & -3.557900 & 1.407352 & 2.128227 \\
\hline 47 & 6 & 0 & -1.642176 & -1.432649 & 3.937266 \\
\hline 48 & 1 & 0 & -0.487694 & -1.611319 & 2.124071 \\
\hline 49 & 6 & 0 & -2.680797 & -0.771854 & 4.582176 \\
\hline 50 & 1 & 0 & -4.183684 & 0.768757 & 4.433686 \\
\hline 51 & 1 & 0 & -1.094211 & -2.222658 & 4.447102 \\
\hline 52 & 1 & 0 & -2.948820 & -1.042557 & 5.602199 \\
\hline 53 & 6 & 0 & 6.049434 & -0.787406 & 0.652658 \\
\hline 54 & 1 & 0 & 6.077942 & -1.502195 & 1.484103 \\
\hline 55 & 1 & 0 & 6.030516 & 0.218793 & 1.085142 \\
\hline 56 & 1 & 0 & 1.888100 & 4.678441 & -2.731085 \\
\hline 57 & 1 & 0 & 2.002774 & -1.233582 & -2.741649 \\
\hline 58 & 1 & 0 & 6.951339 & -0.921944 & 0.049383 \\
\hline 59 & 6 & 0 & 3.338765 & 0.074505 & 1.542497 \\
\hline 60 & 9 & 0 & 3.891315 & 1.296739 & 1.658110 \\
\hline 61 & 9 & 0 & 3.904250 & -0.685574 & 2.491508 \\
\hline 62 & 9 & 0 & 2.045098 & 0.204675 & 1.880753 \\
\hline
\end{tabular}

Zero-point correction

Thermal correction to Energy=

0.467856 (Hartree/Particle)

Thermal correction to Enthalpy=

Thermal correction to Gibbs Free Energy=

Sum of electronic and zero-point Energies=

Sum of electronic and thermal Energies=

Sum of electronic and thermal Enthalpies=

Sum of electronic and thermal Free Energies=

0.503078

0.504022

$-2010.913401$

$-2010.878179$

$-2010.877234$

$-2010.982565$ 
M06 /6-311++G(d,p)-SDD/SMD//M06/6-31G(d)-LANL2DZ energy in toluene solvent $=-2013.050635$

TS2ka

\begin{tabular}{|c|c|c|c|c|c|}
\hline \multirow{2}{*}{$\begin{array}{l}\text { Center } \\
\text { Number }\end{array}$} & \multirow{2}{*}{$\begin{array}{l}\text { Atomic } \\
\text { Number }\end{array}$} & \multirow{2}{*}{$\begin{array}{l}\text { Atomic } \\
\text { Type }\end{array}$} & \multicolumn{3}{|c|}{ Coordinates (Angstroms) } \\
\hline & & & $\mathrm{X}$ & $\mathrm{Y}$ & \\
\hline 1 & 6 & 0 & 0.644468 & 3.539695 & -2.594093 \\
\hline 2 & 6 & 0 & 0.248351 & 2.436880 & -3.340539 \\
\hline 3 & 6 & 0 & 0.150292 & 1.181221 & -2.738526 \\
\hline 4 & 6 & 0 & 0.422398 & 1.032413 & -1.377183 \\
\hline 5 & 6 & 0 & 0.826221 & 2.142760 & -0.633642 \\
\hline 6 & 6 & 0 & 0.940123 & 3.387545 & -1.239508 \\
\hline 7 & 1 & 0 & 0.024412 & 2.544048 & -4.401257 \\
\hline 8 & 1 & 0 & -0.148053 & 0.321586 & -3.340692 \\
\hline 9 & 1 & 0 & 1.041657 & 2.039455 & 0.431350 \\
\hline 10 & 1 & 0 & 1.252021 & 4.247505 & -0.647983 \\
\hline 11 & 53 & 0 & -1.695290 & -3.103845 & -0.197000 \\
\hline 12 & 46 & 0 & -0.414845 & -0.717317 & -0.562218 \\
\hline 13 & 15 & 0 & -2.247190 & 0.459121 & 0.475966 \\
\hline 14 & 6 & 0 & 2.807344 & -0.314167 & -0.492787 \\
\hline 15 & 1 & 0 & 2.813079 & 0.538336 & 0.179938 \\
\hline 16 & 6 & 0 & 1.678831 & -0.724262 & -1.068916 \\
\hline 17 & 6 & 0 & 1.095195 & -1.784951 & -1.721557 \\
\hline 18 & 1 & 0 & 1.228504 & -2.788091 & -1.318897 \\
\hline 19 & 6 & 0 & 4.064250 & -1.043523 & -0.791927 \\
\hline 20 & 8 & 0 & 4.067407 & -2.055305 & -1.479516 \\
\hline 21 & 6 & 0 & -3.726945 & 0.157645 & -0.553407 \\
\hline 22 & 6 & 0 & -4.931768 & -0.318945 & -0.036980 \\
\hline 23 & 6 & 0 & -3.597459 & 0.335491 & -1.936705 \\
\hline 24 & 6 & 0 & -5.998012 & -0.590919 & -0.889062 \\
\hline 25 & 1 & 0 & -5.035198 & -0.499254 & 1.031828 \\
\hline 26 & 6 & 0 & -4.667457 & 0.075004 & -2.782483 \\
\hline 27 & 1 & 0 & -2.644865 & 0.670663 & -2.352146 \\
\hline 28 & 6 & 0 & -5.870797 & -0.390162 & -2.258681 \\
\hline 29 & 1 & 0 & -6.931171 & -0.971858 & -0.477548 \\
\hline 30 & 1 & 0 & -4.556241 & 0.218569 & -3.855874 \\
\hline 31 & 1 & 0 & -6.705704 & -0.609249 & -2.922061 \\
\hline 32 & 6 & 0 & -2.107578 & 2.287548 & 0.580648 \\
\hline 33 & 6 & 0 & -2.612312 & 3.141667 & -0.400412 \\
\hline 34 & 6 & 0 & -1.383450 & 2.833988 & 1.647650 \\
\hline 35 & 6 & 0 & -2.402211 & 4.515020 & -0.313228 \\
\hline 36 & 1 & 0 & -3.183388 & 2.740847 & -1.236490 \\
\hline 37 & 6 & 0 & -1.183283 & 4.205537 & 1.738465 \\
\hline 38 & 1 & 0 & -0.981854 & 2.177232 & 2.421488 \\
\hline 39 & 6 & 0 & -1.692766 & 5.050882 & 0.755557 \\
\hline 40 & 1 & 0 & -2.805078 & 5.169583 & -1.084480 \\
\hline 41 & 1 & 0 & -0.629780 & 4.616483 & 2.581462 \\
\hline 42 & 1 & 0 & -1.538074 & 6.126400 & 0.825681 \\
\hline 43 & 6 & 0 & -2.697597 & 0.036955 & 2.195905 \\
\hline 44 & 6 & 0 & -3.701232 & 0.756063 & 2.859714 \\
\hline 45 & 6 & 0 & -2.009030 & -0.966051 & 2.879601 \\
\hline 46 & 6 & 0 & -4.023146 & 0.457325 & 4.177034 \\
\hline 47 & 1 & 0 & -4.226418 & 1.560031 & 2.342184 \\
\hline 48 & 6 & 0 & -2.328624 & -1.257157 & 4.203226 \\
\hline 49 & 1 & 0 & -1.231283 & -1.530543 & 2.366153 \\
\hline 50 & 6 & 0 & -3.336229 & -0.551522 & 4.849832 \\
\hline 51 & 1 & 0 & -4.808895 & 1.015977 & 4.682876 \\
\hline 52 & 1 & 0 & -1.787855 & -2.044140 & 4.725557 \\
\hline 53 & 1 & 0 & -3.586772 & -0.783270 & 5.883889 \\
\hline 54 & 1 & 0 & 0.732021 & 4.517280 & -3.065375 \\
\hline 55 & 1 & 0 & 0.775283 & -1.716762 & -2.761793 \\
\hline 56 & 6 & 0 & 5.325461 & -0.523571 & -0.200871 \\
\hline 57 & 6 & 0 & 5.457798 & 0.738271 & 0.396524 \\
\hline 58 & 6 & 0 & 6.453751 & -1.345163 & -0.264441 \\
\hline 59 & 6 & 0 & 6.669184 & 1.154538 & 0.915300 \\
\hline 60 & 1 & 0 & 4.613450 & 1.423884 & 0.439958 \\
\hline 61 & 6 & 0 & 7.674299 & -0.946601 & 0.258967 \\
\hline 62 & 1 & 0 & 6.347140 & -2.318744 & -0.738946 \\
\hline 63 & 6 & 0 & 7.785076 & 0.311677 & 0.858215 \\
\hline 64 & 1 & 0 & 6.787610 & 2.133908 & 1.374261 \\
\hline 65 & 1 & 0 & 8.528610 & -1.616198 & 0.198776 \\
\hline 66 & 8 & 0 & 8.918344 & 0.801248 & 1.405405 \\
\hline 67 & 6 & 0 & 10.070199 & -0.007231 & 1.375561 \\
\hline 68 & 1 & 0 & 10.856570 & 0.557772 & 1.88200 \\
\hline 69 & 1 & 0 & 9.909247 & -0.957718 & 1.905324 \\
\hline 70 & 1 & 0 & 10.386051 & -0.221115 & 0.343750 \\
\hline
\end{tabular}

Zero-point correction=

547827 (Hartree/Particle)

Thermal correction to Energy=

Thermal correction to Enthalpy=

Thermal correction to Gibbs Free Energy=

Sum of electronic and zero-point Energies=

Sum of electronic and thermal Energies=

Sum of electronic and thermal Enthalpies=

Sum of electronic and thermal Free Energies

0.585598

0.586543

0.473096

$-1979.945983$

$-1979.90821$

$-1979.908211$

$-1980.020713$

M06 /6-311++G(d,p)-SDD/SMD//M06/6-31G(d)-LANL2DZ energy in toluene solvent $=-1982.1553042$

\section{TS2la}

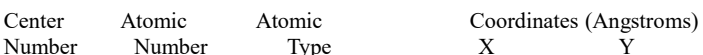

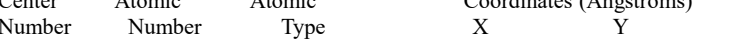




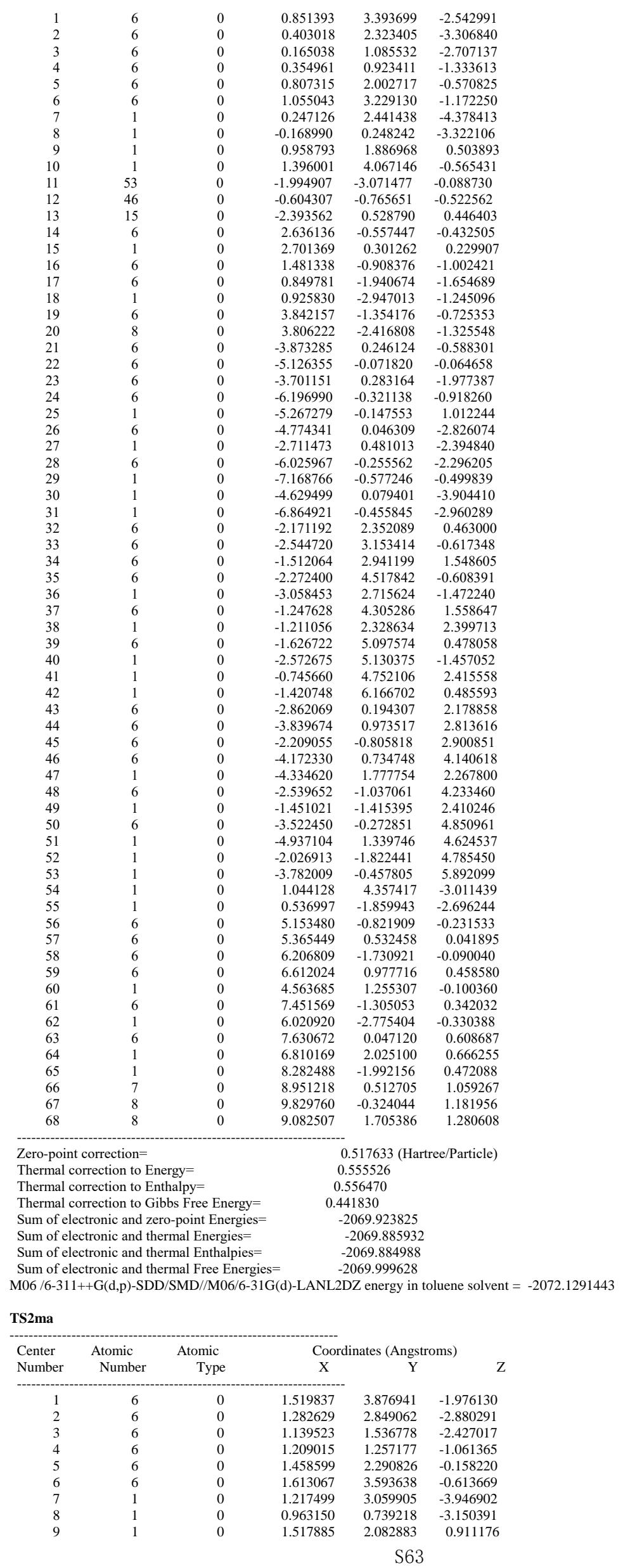




\begin{tabular}{|c|c|c|c|c|c|}
\hline 10 & 1 & 0 & 1.796276 & 4.394175 & 0.102015 \\
\hline 11 & 53 & 0 & -0.820261 & -3.060209 & -0.605524 \\
\hline 12 & 46 & 0 & 0.367932 & -0.606114 & -0.559971 \\
\hline 13 & 15 & 0 & -1.630843 & 0.369979 & 0.365389 \\
\hline 14 & 6 & 0 & 3.472804 & -0.129555 & 0.111107 \\
\hline 15 & 1 & 0 & 3.334595 & 0.618273 & 0.888414 \\
\hline 16 & 6 & 0 & 2.489351 & -0.497853 & -0.714367 \\
\hline 17 & 6 & 0 & 2.097166 & -1.480207 & -1.590381 \\
\hline 18 & 1 & 0 & 2.217658 & -2.525033 & -1.304470 \\
\hline 19 & 6 & 0 & 4.794698 & -0.734711 & -0.057776 \\
\hline 20 & 8 & 0 & 5.116852 & -1.550228 & -0.892379 \\
\hline 21 & 6 & 0 & -3.013405 & 0.067438 & -0.790398 \\
\hline 22 & 6 & 0 & -4.248652 & -0.435005 & -0.381482 \\
\hline 23 & 6 & 0 & -2.783922 & 0.294225 & -2.152941 \\
\hline 24 & 6 & 0 & -5.247978 & -0.680265 & -1.318535 \\
\hline 25 & 1 & 0 & -4.430349 & -0.658323 & 0.668411 \\
\hline 26 & 6 & 0 & -3.787296 & 0.059237 & -3.084016 \\
\hline 27 & 1 & 0 & -1.804870 & 0.642787 & -2.488168 \\
\hline 28 & 6 & 0 & -5.022802 & -0.428057 & -2.666769 \\
\hline 29 & 1 & 0 & -6.205598 & -1.079769 & -0.989265 \\
\hline 30 & 1 & 0 & -3.598046 & 0.240566 & -4.140546 \\
\hline 31 & 1 & 0 & -5.805633 & -0.623776 & -3.397459 \\
\hline 32 & 6 & 0 & -1.610904 & 2.188967 & 0.614209 \\
\hline 33 & 6 & 0 & -2.008782 & 3.077608 & -0.385814 \\
\hline 34 & 6 & 0 & -1.080479 & 2.699634 & 1.804936 \\
\hline 35 & 6 & 0 & -1.885830 & 4.450491 & -0.195285 \\
\hline 36 & 1 & 0 & -2.426338 & 2.701282 & -1.318989 \\
\hline 37 & 6 & 0 & -0.969235 & 4.071094 & 1.997693 \\
\hline 38 & 1 & 0 & -0.756805 & 2.018159 & 2.592582 \\
\hline 39 & 6 & 0 & -1.371955 & 4.950513 & 0.996418 \\
\hline 40 & 1 & 0 & -2.202553 & 5.132334 & -0.982852 \\
\hline 41 & 1 & 0 & -0.567732 & 4.454052 & 2.934698 \\
\hline 42 & 1 & 0 & -1.285332 & 6.025564 & 1.145780 \\
\hline 43 & 6 & 0 & -2.148666 & -0.228390 & 2.011457 \\
\hline 44 & 6 & 0 & -3.222429 & 0.373575 & 2.682844 \\
\hline 45 & 6 & 0 & -1.437683 & -1.252414 & 2.639182 \\
\hline 46 & 6 & 0 & -3.587802 & -0.060520 & 3.950250 \\
\hline 47 & 1 & 0 & -3.765300 & 1.194969 & 2.213514 \\
\hline 48 & 6 & 0 & -1.802737 & -1.680595 & 3.912575 \\
\hline 49 & 1 & 0 & -0.605419 & -1.727042 & 2.120137 \\
\hline 50 & 6 & 0 & -2.877252 & -1.089252 & 4.565953 \\
\hline 51 & 1 & 0 & -4.424928 & 0.410513 & 4.462706 \\
\hline 52 & 1 & 0 & -1.243529 & -2.482055 & 4.391354 \\
\hline 53 & 1 & 0 & -3.162388 & -1.425664 & 5.561430 \\
\hline 54 & 6 & 0 & 5.819620 & -0.241793 & 0.980915 \\
\hline 55 & 1 & 0 & 1.640000 & 4.899215 & -2.330622 \\
\hline 56 & 1 & 0 & 1.942052 & -1.280411 & -2.650459 \\
\hline 57 & 9 & 0 & 5.943154 & 1.089207 & 0.901733 \\
\hline 58 & 9 & 0 & 5.383161 & -0.529982 & 2.213605 \\
\hline 59 & 9 & 0 & 7.007187 & -0.790214 & 0.811222 \\
\hline
\end{tabular}

Zero-point correction $=$ 0.438440 (Hartree/Particle) Thermal correction to Energy= Thermal correction to Enthalpy

Thermal correction to Gibbs Free Energy=

Sum of electronic and zero-point Energies

Sum of electronic and thermal Energies $=$

Sum of electronic and thermal Enthalpies=

Sum of electronic and thermal Free Energies=

$$
0.472886
$$

$$
\begin{gathered}
0.472886 \\
0.473830 \\
0.366653
\end{gathered}
$$$$
-1971.650675
$$$$
-1971.61622
$$$$
-1971.615285
$$$$
-1971.722462
$$

M06 /6-311++G(d,p)-SDD/SMD//M06/6-31G(d)-LANL2DZ energy in toluene solvent $=-1973.7513506$

TS2ab

\begin{tabular}{rrrrrr} 
Center & Atomic & Atomic & \multicolumn{2}{c}{ Coordinates (Angstroms) } \\
Number & Number & Type & X & Y & $Z$ \\
-1 & 6 & 0 & -4.075317 & -0.989028 & -0.532870 \\
2 & 6 & 0 & -3.518153 & -0.386617 & -1.652225 \\
3 & 6 & 0 & -2.403288 & 0.427730 & -1.485553 \\
4 & 6 & 0 & -1.842295 & 0.614382 & -0.216986 \\
5 & 6 & 0 & -2.447967 & 0.018059 & 0.896246 \\
6 & 6 & 0 & -3.564791 & -0.784719 & 0.746541 \\
7 & 1 & 0 & -3.954564 & -0.566379 & -2.630921 \\
8 & 1 & 0 & -1.961879 & 0.905388 & -2.360852 \\
9 & 1 & 0 & -2.021325 & 0.159461 & 1.890316 \\
10 & 1 & 0 & -4.026960 & -1.285242 & 1.593143 \\
11 & 53 & 0 & 2.701083 & 2.040137 & -0.800479 \\
12 & 46 & 0 & 0.175807 & 1.200746 & -0.235818 \\
13 & 15 & 0 & 1.003211 & -1.011959 & 0.265458 \\
14 & 6 & 0 & -2.112140 & 3.148663 & 1.043005 \\
15 & 1 & 0 & -2.430883 & 2.519004 & 1.872247 \\
16 & 6 & 0 & -1.358319 & 2.689488 & 0.045026 \\
17 & 6 & 0 & -0.560612 & 3.113503 & -0.994472 \\
18 & 1 & 0 & 0.167489 & 3.903494 & -0.814037 \\
19 & 6 & 0 & -2.568500 & 4.563784 & 1.006022 \\
20 & 8 & 0 & -2.289098 & 5.321234 & 0.097803 \\
21 & 6 & 0 & 1.855178 & -1.639058 & -1.225129 \\
22 & 6 & 0 & 3.118291 & -2.230226 & -1.189716 \\
23 & 6 & 0 & 1.223814 & -1.449430 & -2.460298 \\
24 & 6 & 0 & 3.725514 & -2.651851 & -2.368585 \\
25 & 1 & 0 & 3.643896 & -2.342447 & -0.243165 \\
26 & 6 & 0 & 1.825940 & -1.883402 & -3.633718 \\
27 & 1 & 0 & 0.261574 & -0.935068 & -2.504050
\end{tabular}




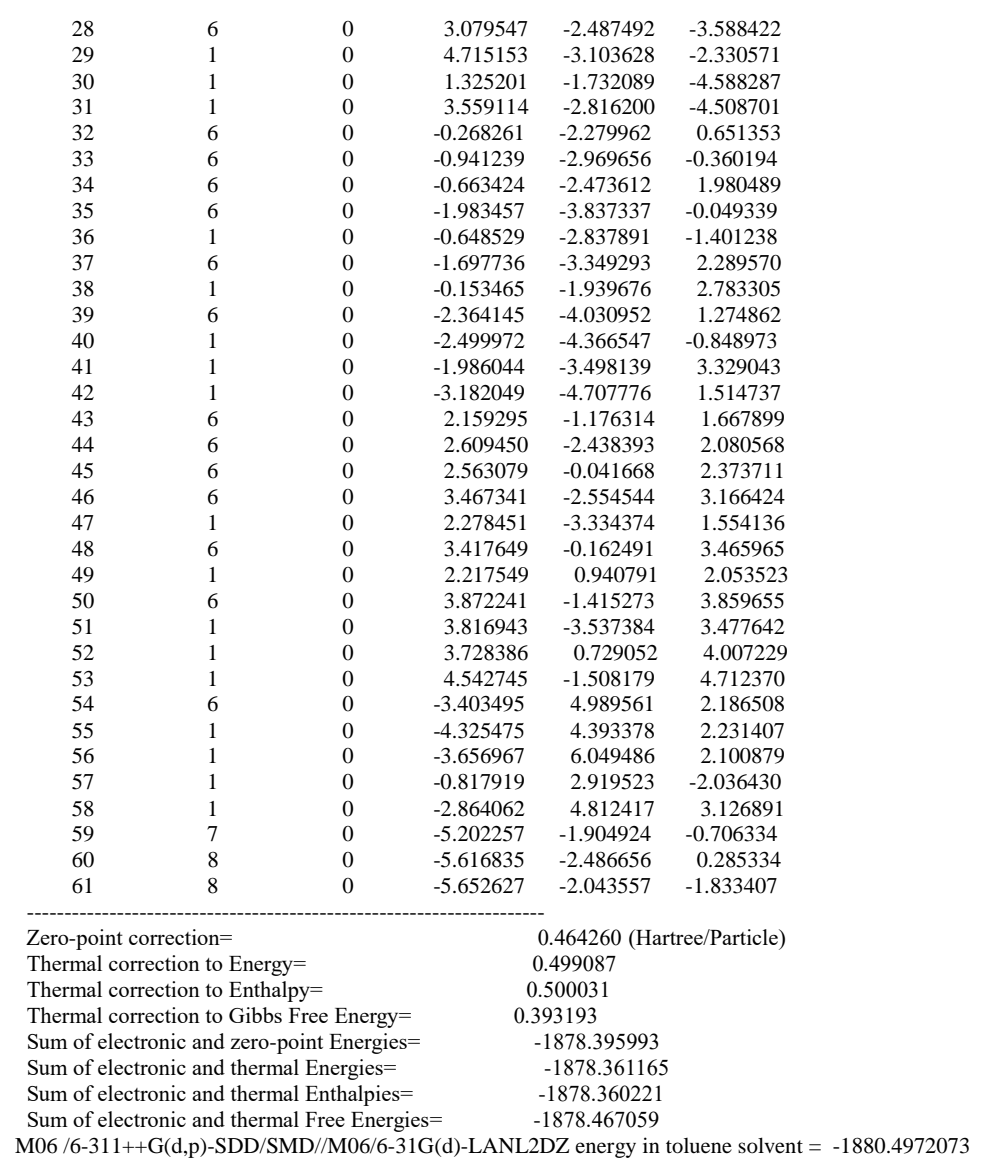

\section{TS2ac}

\begin{tabular}{|c|c|c|c|c|c|}
\hline \multirow{2}{*}{$\begin{array}{l}\text { Center } \\
\text { Number }\end{array}$} & \multirow{2}{*}{$\begin{array}{l}\text { Atomic } \\
\text { Number }\end{array}$} & \multirow{2}{*}{$\begin{array}{l}\text { Atomic } \\
\text { Type }\end{array}$} & \multicolumn{3}{|c|}{ Coordinates (Angstroms) } \\
\hline & & & $\mathrm{X}$ & $\mathrm{Y}$ & 7 \\
\hline 1 & 6 & 0 & -4.022493 & -1.708216 & -0.401300 \\
\hline 2 & 6 & 0 & -3.548091 & -1.077469 & -1.548550 \\
\hline 3 & 6 & 0 & -2.542269 & -0.111940 & -1.435304 \\
\hline 4 & 6 & 0 & -1.989319 & 0.209102 & -0.199213 \\
\hline 5 & 6 & 0 & -2.493821 & -0.418300 & 0.946829 \\
\hline 6 & 6 & 0 & -3.503614 & -1.354808 & 0.851756 \\
\hline 7 & 1 & 0 & -3.946039 & -1.317939 & -2.531588 \\
\hline 8 & 1 & 0 & -2.188514 & 0.376579 & -2.344771 \\
\hline 9 & 1 & 0 & -2.074579 & -0.185652 & 1.927540 \\
\hline 10 & 1 & 0 & -3.890427 & -1.867906 & 1.730735 \\
\hline 11 & 53 & 0 & 2.248811 & 2.372086 & -0.884338 \\
\hline 12 & 46 & 0 & -0.112429 & 1.137667 & -0.274447 \\
\hline 13 & 15 & 0 & 1.058197 & -0.898448 & 0.283243 \\
\hline 14 & 6 & 0 & -2.584841 & 2.761611 & 1.058821 \\
\hline 15 & 1 & 0 & -2.725485 & 2.117611 & 1.925750 \\
\hline 16 & 6 & 0 & -1.836798 & 2.397314 & 0.019076 \\
\hline 17 & 6 & 0 & -1.178762 & 2.867952 & -1.090242 \\
\hline 18 & 1 & 0 & -0.556493 & 3.757695 & -1.002552 \\
\hline 19 & 6 & 0 & -3.285532 & 4.068966 & 1.020699 \\
\hline 20 & 8 & 0 & -3.219429 & 4.831130 & 0.075273 \\
\hline 21 & 6 & 0 & 2.013323 & -1.412757 & -1.190851 \\
\hline 22 & 6 & 0 & 3.344051 & -1.825922 & -1.132990 \\
\hline 23 & 6 & 0 & 1.383115 & -1.312056 & -2.437084 \\
\hline 24 & 6 & 0 & 4.023672 & -2.161373 & -2.300027 \\
\hline 25 & 1 & 0 & 3.865163 & -1.865391 & -0.178266 \\
\hline 26 & 6 & 0 & 2.059462 & -1.659467 & -3.598770 \\
\hline 27 & 1 & 0 & 0.362132 & -0.929700 & -2.497485 \\
\hline 28 & 6 & 0 & 3.382605 & -2.086746 & -3.531089 \\
\hline 29 & 1 & 0 & 5.065186 & -2.473308 & -2.244690 \\
\hline 30 & 1 & 0 & 1.560041 & -1.574702 & -4.562322 \\
\hline 31 & 1 & 0 & 3.919624 & -2.345238 & -4.442132 \\
\hline 32 & 6 & 0 & 0.011707 & -2.355275 & 0.686678 \\
\hline 33 & 6 & 0 & -0.576890 & -3.115458 & -0.327372 \\
\hline 34 & 6 & 0 & -0.313884 & -2.639718 & 2.017560 \\
\hline 35 & 6 & 0 & -1.466789 & -4.137850 & -0.018360 \\
\hline 36 & 1 & 0 & -0.337395 & -2.912410 & -1.370446 \\
\hline 37 & 6 & 0 & -1.193216 & -3.671988 & 2.325084 \\
\hline 38 & 1 & 0 & 0.125651 & -2.053060 & 2.824346 \\
\hline 39 & 6 & 0 & -1.776062 & -4.421577 & 1.307952 \\
\hline 40 & 1 & 0 & -1.922302 & -4.715725 & -0.821727 \\
\hline 41 & 1 & 0 & -1.426922 & -3.887911 & 3.366665 \\
\hline 42 & 1 & 0 & -2.472020 & -5.223614 & 1.547883 \\
\hline 43 & 6 & 0 & 2.218777 & -0.837521 & 1.691640 \\
\hline
\end{tabular}




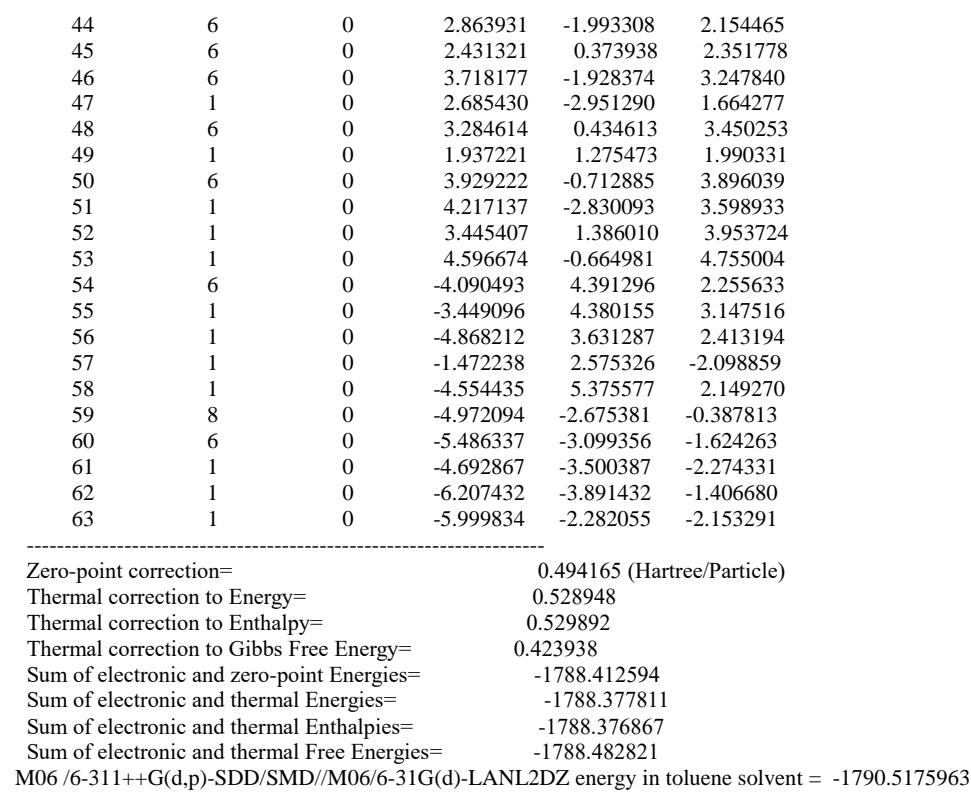

TS6ba

\begin{tabular}{|c|c|c|c|c|c|}
\hline \multirow{2}{*}{$\begin{array}{l}\text { Center } \\
\text { Number }\end{array}$} & \multirow{2}{*}{$\begin{array}{l}\text { Atomic } \\
\text { Number }\end{array}$} & \multirow{2}{*}{$\begin{array}{l}\text { Atomic } \\
\text { Type }\end{array}$} & \multicolumn{3}{|c|}{ Coordinates (Angstroms) } \\
\hline & & & $\mathrm{X}$ & $\mathrm{Y}$ & $z$ \\
\hline 1 & 6 & 0 & -1.533336 & 4.123467 & -1.641496 \\
\hline 2 & 6 & 0 & -1.255908 & 3.817449 & -0.311565 \\
\hline 3 & 6 & 0 & -0.919701 & 2.515300 & 0.053374 \\
\hline 4 & 6 & 0 & -0.837685 & 1.503117 & -0.906354 \\
\hline 5 & 6 & 0 & -1.116982 & 1.818080 & -2.238111 \\
\hline 6 & 6 & 0 & -1.462085 & 3.119961 & -2.604169 \\
\hline 7 & 1 & 0 & -1.302827 & 4.595779 & 0.450545 \\
\hline 8 & 1 & 0 & -0.711059 & 2.294674 & 1.103303 \\
\hline 9 & 1 & 0 & -1.072912 & 1.041522 & -3.004230 \\
\hline 10 & 1 & 0 & -1.679036 & 3.348218 & -3.648208 \\
\hline 11 & 53 & 0 & 0.013625 & -3.049471 & 0.424392 \\
\hline 12 & 46 & 0 & -0.380772 & -0.411571 & -0.356304 \\
\hline 13 & 15 & 0 & 1.885466 & 0.208659 & 0.029102 \\
\hline 14 & 6 & 0 & -2.915917 & -1.333817 & -1.935891 \\
\hline 15 & 1 & 0 & -2.445172 & -1.386391 & -2.915251 \\
\hline 16 & 6 & 0 & -2.331358 & -0.913228 & -0.793278 \\
\hline 17 & 6 & 0 & -3.109412 & -0.954780 & 0.387230 \\
\hline 18 & 1 & 0 & -2.933660 & -1.830127 & 1.022999 \\
\hline 19 & 6 & 0 & -4.262301 & -1.820740 & -1.701156 \\
\hline 20 & 8 & 0 & -4.718417 & -1.788917 & -0.544363 \\
\hline 21 & 6 & 0 & 2.477779 & -0.290960 & 1.684175 \\
\hline 22 & 6 & 0 & 3.794403 & -0.676261 & 1.937401 \\
\hline 23 & 6 & 0 & 1.565320 & -0.222263 & 2.741473 \\
\hline 24 & 6 & 0 & 4.194083 & -0.981553 & 3.235028 \\
\hline 25 & 1 & 0 & 4.509915 & -0.750390 & 1.119065 \\
\hline 26 & 6 & 0 & 1.969487 & -0.515155 & 4.037538 \\
\hline 27 & 1 & 0 & 0.527361 & 0.047974 & 2.535128 \\
\hline 28 & 6 & 0 & 3.285797 & -0.896569 & 4.284721 \\
\hline 29 & 1 & 0 & 5.220677 & -1.290755 & 3.424858 \\
\hline 30 & 1 & 0 & 1.251881 & -0.460910 & 4.854310 \\
\hline 31 & 1 & 0 & 3.600794 & -1.138066 & 5.298665 \\
\hline 32 & 6 & 0 & 3.084380 & -0.465561 & -1.177317 \\
\hline 33 & 6 & 0 & 2.738828 & -1.584537 & -1.939126 \\
\hline 34 & 6 & 0 & 4.346828 & 0.117056 & -1.347564 \\
\hline 35 & 6 & 0 & 3.646170 & -2.117264 & -2.850022 \\
\hline 36 & 1 & 0 & 1.759919 & -2.045738 & -1.802232 \\
\hline 37 & 6 & 0 & 5.251183 & -0.420571 & -2.255364 \\
\hline 38 & 1 & 0 & 4.619873 & 1.001879 & -0.771367 \\
\hline 39 & 6 & 0 & 4.900633 & -1.538960 & -3.007916 \\
\hline 40 & 1 & 0 & 3.367730 & -2.990832 & -3.436917 \\
\hline 41 & 1 & 0 & 6.230954 & 0.038147 & -2.379565 \\
\hline 42 & 1 & 0 & 5.608585 & -1.957186 & -3.722016 \\
\hline 43 & 6 & 0 & 2.242341 & 2.006961 & -0.007603 \\
\hline 44 & 6 & 0 & 2.519251 & 2.732938 & 1.151153 \\
\hline 45 & 6 & 0 & 2.121795 & 2.685357 & -1.226156 \\
\hline 46 & 6 & 0 & 2.663096 & 4.117491 & 1.094393 \\
\hline 47 & 1 & 0 & 2.620629 & 2.218643 & 2.106630 \\
\hline 48 & 6 & 0 & 2.266132 & 4.063557 & -1.280511 \\
\hline 49 & 1 & 0 & 1.889797 & 2.129302 & -2.135418 \\
\hline 50 & 6 & 0 & 2.533120 & 4.784290 & -0.117705 \\
\hline 51 & 1 & 0 & 2.880321 & 4.673883 & 2.005027 \\
\hline 52 & 1 & 0 & 2.154478 & 4.579506 & -2.232855 \\
\hline 53 & 1 & 0 & 2.639430 & 5.867199 & -0.160011 \\
\hline 54 & 6 & 0 & -5.080156 & -2.367650 & -2.825859 \\
\hline 55 & 1 & 0 & -5.213289 & -1.598321 & -3.597699 \\
\hline 56 & 1 & 0 & -6.056411 & -2.706881 & -2.470706 \\
\hline 57 & 1 & 0 & -4.547932 & -3.203504 & -3.298177 \\
\hline
\end{tabular}




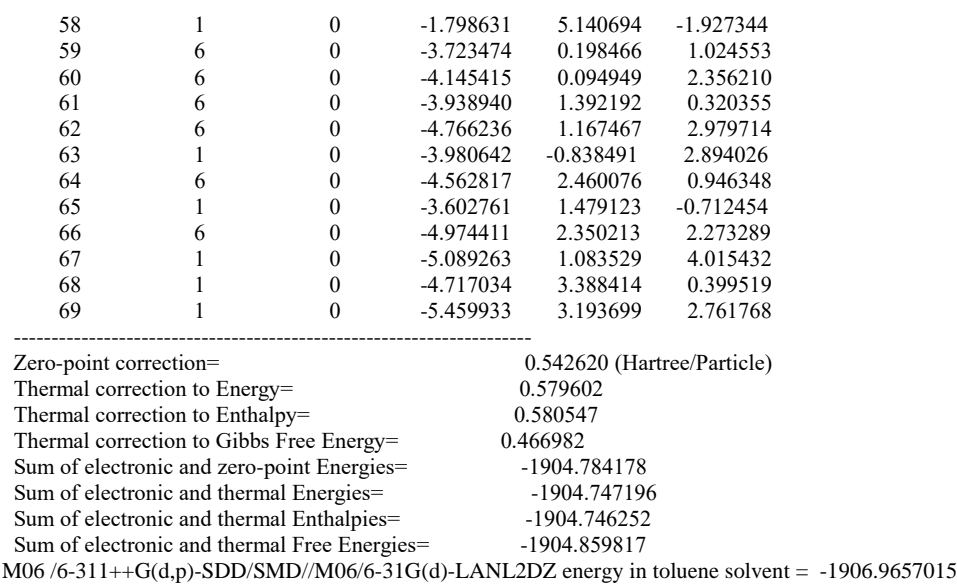

\section{TS6ca}

\begin{tabular}{|c|c|c|c|c|c|}
\hline \multirow{2}{*}{$\begin{array}{l}\text { Center } \\
\text { Number }\end{array}$} & \multirow{2}{*}{$\begin{array}{l}\text { Atomic } \\
\text { Number }\end{array}$} & \multirow{2}{*}{$\begin{array}{l}\text { Atomic } \\
\text { Type }\end{array}$} & \multicolumn{3}{|c|}{ Coordinates (Angstroms) } \\
\hline & & & $\mathrm{X}$ & $\mathrm{Y}$ & \\
\hline 1 & 6 & 0 & -1.715154 & 3.213927 & -2.729470 \\
\hline 2 & 6 & 0 & -1.621295 & 3.214390 & -1.340277 \\
\hline 3 & 6 & 0 & -1.102019 & 2.107045 & -0.672142 \\
\hline 4 & 6 & 0 & -0.656985 & 0.988398 & -1.381139 \\
\hline 5 & 6 & 0 & -0.756569 & 0.994532 & -2.774265 \\
\hline 6 & 6 & 0 & -1.279298 & 2.101391 & -3.444841 \\
\hline 7 & 1 & 0 & -1.949157 & 4.083412 & -0.769091 \\
\hline 8 & 1 & 0 & -1.031174 & 2.127140 & 0.418114 \\
\hline 9 & 1 & 0 & -0.432019 & 0.125175 & -3.349393 \\
\hline 10 & 1 & 0 & -1.350409 & 2.089943 & -4.532891 \\
\hline 11 & 53 & 0 & 0.792114 & -2.956812 & 0.981172 \\
\hline 12 & 46 & 0 & 0.063183 & -0.644250 & -0.384248 \\
\hline 13 & 15 & 0 & 2.087254 & 0.515157 & 0.112079 \\
\hline 14 & 6 & 0 & -1.944504 & -2.358347 & -2.031291 \\
\hline 15 & 1 & 0 & -1.310407 & -2.468296 & -2.908330 \\
\hline 16 & 6 & 0 & -1.659641 & -1.622062 & -0.932249 \\
\hline 17 & 6 & 0 & -2.622239 & -1.649573 & 0.114526 \\
\hline 18 & 1 & 0 & -2.387961 & -2.346024 & 0.927653 \\
\hline 19 & 6 & 0 & -3.179189 & -3.096362 & -1.878006 \\
\hline 20 & 8 & 0 & -3.824379 & -2.966863 & -0.819698 \\
\hline 21 & 6 & 0 & 2.559116 & 0.434353 & 1.876069 \\
\hline 22 & 6 & 0 & 3.884733 & 0.495761 & 2.307291 \\
\hline 23 & 6 & 0 & 1.529835 & 0.377649 & 2.819713 \\
\hline 24 & 6 & 0 & 4.175305 & 0.505586 & 3.667982 \\
\hline 25 & 1 & 0 & 4.694362 & 0.527409 & 1.578188 \\
\hline 26 & 6 & 0 & 1.821311 & 0.401502 & 4.178091 \\
\hline 27 & 1 & 0 & 0.496341 & 0.295444 & 2.477181 \\
\hline 28 & 6 & 0 & 3.145555 & 0.463130 & 4.602723 \\
\hline 29 & 1 & 0 & 5.212089 & 0.543110 & 3.998446 \\
\hline 30 & 1 & 0 & 1.014270 & 0.353379 & 4.907059 \\
\hline 31 & 1 & 0 & 3.376602 & 0.469081 & 5.666848 \\
\hline 32 & 6 & 0 & 3.549860 & -0.060185 & -0.826009 \\
\hline 33 & 6 & 0 & 3.580483 & -1.374880 & -1.299497 \\
\hline 34 & 6 & 0 & 4.645645 & 0.778981 & -1.063701 \\
\hline 35 & 6 & 0 & 4.692400 & -1.842843 & -1.993263 \\
\hline 36 & 1 & 0 & 2.733245 & -2.034917 & -1.109543 \\
\hline 37 & 6 & 0 & 5.753454 & 0.307621 & -1.758785 \\
\hline 38 & 1 & 0 & 4.627765 & 1.810776 & -0.711932 \\
\hline 39 & 6 & 0 & 5.777320 & -1.004414 & -2.224543 \\
\hline 40 & 1 & 0 & 4.706215 & -2.869595 & -2.354600 \\
\hline 41 & 1 & 0 & 6.599150 & 0.968961 & -1.940696 \\
\hline 42 & 1 & 0 & 6.644350 & -1.371471 & -2.771804 \\
\hline 43 & 6 & 0 & 2.061184 & 2.319323 & -0.213897 \\
\hline 44 & 6 & 0 & 1.947287 & 3.249500 & 0.820279 \\
\hline 45 & 6 & 0 & 2.024213 & 2.767343 & -1.539618 \\
\hline 46 & 6 & 0 & 1.791111 & 4.603631 & 0.533921 \\
\hline 47 & 1 & 0 & 1.975649 & 2.918268 & 1.858087 \\
\hline 48 & 6 & 0 & 1.875487 & 4.117589 & -1.822528 \\
\hline 49 & 1 & 0 & 2.095668 & 2.048876 & -2.356978 \\
\hline 50 & 6 & 0 & 1.752677 & 5.039486 & -0.784836 \\
\hline 51 & 1 & 0 & 1.702148 & 5.319961 & 1.349387 \\
\hline 52 & 1 & 0 & 1.840455 & 4.450108 & -2.858732 \\
\hline 53 & 1 & 0 & 1.626748 & 6.098063 & -1.007281 \\
\hline 54 & 6 & 0 & -3.657353 & -4.029758 & -2.942009 \\
\hline 55 & 1 & 0 & -4.697358 & -4.318212 & -2.768100 \\
\hline 56 & 1 & 0 & -3.032576 & -4.933227 & -2.929404 \\
\hline 57 & 1 & 0 & -3.548265 & -3.576581 & -3.934686 \\
\hline 58 & 1 & 0 & -2.122380 & 4.078158 & -3.253030 \\
\hline 59 & 6 & 0 & -3.538075 & -0.575201 & 0.427154 \\
\hline 60 & 6 & 0 & -4.166878 & -0.549112 & 1.676436 \\
\hline 61 & 6 & 0 & -3.858311 & 0.420942 & -0.514813 \\
\hline 62 & 6 & 0 & -5.083917 & 0.439269 & 2.000579 \\
\hline 63 & 1 & 0 & -3.929407 & -1.322825 & 2.406292 \\
\hline 64 & 6 & 0 & -4.773180 & 1.402928 & -0.207160 \\
\hline 65 & 1 & 0 & -3.365037 & 0.420587 & -1.486699 \\
\hline
\end{tabular}




\begin{tabular}{|c|c|c|c|c|c|}
\hline 66 & 6 & 0 & -5.390261 & 1.421449 & 1.052931 \\
\hline 67 & 1 & 0 & -5.553477 & 0.440104 & 2.980471 \\
\hline 68 & 1 & 0 & -5.022498 & 2.188535 & -0.917043 \\
\hline 69 & 8 & 0 & -6.262465 & 2.427251 & 1.259165 \\
\hline 70 & 6 & 0 & -6.885612 & 2.520104 & 2.519143 \\
\hline 71 & 1 & 0 & -6.145321 & 2.646103 & 3.322746 \\
\hline 72 & 1 & 0 & -7.528424 & 3.402618 & 2.479761 \\
\hline 73 & 1 & 0 & -7.500668 & 1.633467 & 2.731412 \\
\hline \multicolumn{4}{|c|}{ Zero-point correction $=$} & \multicolumn{2}{|c|}{0.575648 (Hartree/Particle) } \\
\hline \multicolumn{4}{|c|}{ Thermal correction to Energy $=$} & \multicolumn{2}{|c|}{0.614998} \\
\hline \multicolumn{4}{|c|}{ Thermal correction to Enthalpy= } & \multicolumn{2}{|c|}{0.615943} \\
\hline \multirow{2}{*}{\multicolumn{4}{|c|}{$\begin{array}{l}\text { Thermal correction to Gibbs Free Energy= } \\
\text { Sum of electronic and zero-point Energies= }\end{array}$}} & \multicolumn{2}{|c|}{.499426} \\
\hline \multirow{2}{*}{\multicolumn{4}{|c|}{$\begin{array}{l}\text { Sum of electronic and zero-point Energies= } \\
\text { Sum of electronic and thermal Energies }=\end{array}$}} & \multicolumn{2}{|c|}{-2019.215680} \\
\hline & & & & \multirow{2}{*}{\multicolumn{2}{|c|}{$\begin{array}{r}-2019.176330 \\
-2019175386\end{array}$}} \\
\hline \multicolumn{4}{|c|}{ Sum of electronic and thermal Enthalpies= } & & \\
\hline \multicolumn{4}{|c|}{ Sum of electronic and thermal Free Energies } & \multicolumn{2}{|c|}{-2019.1191903} \\
\hline
\end{tabular}

Sum of electronic and thermal Free Energies $=\quad-2019.291903$

M06 /6-311++G(d,p)-SDD/SMD//M06/6-31G(d)-LANL2DZ energy in toluene solvent = -2021.462684

TS6d

\begin{tabular}{|c|c|c|c|c|c|}
\hline \multirow{2}{*}{$\begin{array}{l}\text { Center } \\
\text { Number }\end{array}$} & \multirow{2}{*}{$\begin{array}{l}\text { Atomic } \\
\text { Number }\end{array}$} & \multirow{2}{*}{$\begin{array}{l}\text { Atomic } \\
\text { Type }\end{array}$} & \multicolumn{3}{|c|}{ Coordinates (Angstroms) } \\
\hline & & & $\mathrm{X}$ & $\mathrm{Y}$ & \\
\hline 1 & 6 & 0 & -2.009816 & 2.973851 & -2.626635 \\
\hline 2 & 6 & 0 & -1.872186 & 2.977768 & -1.241083 \\
\hline 3 & 6 & 0 & -1.217443 & 1.928141 & -0.599222 \\
\hline 4 & 6 & 0 & -0.682720 & 0.866446 & -1.332401 \\
\hline 5 & 6 & 0 & -0.819123 & 0.871022 & -2.722193 \\
\hline 6 & 6 & 0 & -1.479401 & 1.919225 & -3.365538 \\
\hline 7 & 1 & 0 & -2.275436 & 3.802582 & -0.653287 \\
\hline 8 & 1 & 0 & -1.115438 & 1.950165 & 0.488694 \\
\hline 9 & 1 & 0 & -0.416257 & 0.048000 & -3.315764 \\
\hline 10 & 1 & 0 & -1.581229 & 1.907676 & -4.450982 \\
\hline 11 & 53 & 0 & 1.260522 & -2.929954 & 0.908540 \\
\hline 12 & 46 & 0 & 0.222211 & -0.701113 & -0.382671 \\
\hline 13 & 15 & 0 & 2.114078 & 0.667208 & 0.087927 \\
\hline 14 & 6 & 0 & -1.666286 & -2.683890 & -1.937159 \\
\hline 15 & 1 & 0 & -1.068915 & -2.789746 & -2.839692 \\
\hline 16 & 6 & 0 & -1.396170 & -1.879904 & -0.887898 \\
\hline 17 & 6 & 0 & -2.283382 & -1.908324 & 0.205305 \\
\hline 18 & 1 & 0 & -1.964405 & -2.521452 & 1.055456 \\
\hline 19 & 6 & 0 & -2.838555 & -3.506822 & -1.687653 \\
\hline 20 & 8 & 0 & -3.449990 & -3.376125 & -0.612837 \\
\hline 21 & 6 & 0 & 2.583383 & 0.600507 & 1.851638 \\
\hline 22 & 6 & 0 & 3.906637 & 0.658359 & 2.288144 \\
\hline 23 & 6 & 0 & 1.549934 & 0.534812 & 2.791353 \\
\hline 24 & 6 & 0 & 4.190759 & 0.657471 & 3.650803 \\
\hline 25 & 1 & 0 & 4.720033 & 0.693755 & 1.564000 \\
\hline 26 & 6 & 0 & 1.835012 & 0.549447 & 4.150416 \\
\hline 27 & 1 & 0 & 0.516545 & 0.457719 & 2.445522 \\
\hline 28 & 6 & 0 & 3.158053 & 0.608096 & 4.580890 \\
\hline 29 & 1 & 0 & 5.226148 & 0.691822 & 3.985516 \\
\hline 30 & 1 & 0 & 1.025135 & 0.497660 & 4.875904 \\
\hline 31 & 1 & 0 & 3.384475 & 0.606494 & 5.645858 \\
\hline 32 & 6 & 0 & 3.614514 & 0.249410 & -0.868909 \\
\hline 33 & 6 & 0 & 3.737973 & -1.022350 & -1.433813 \\
\hline 34 & 6 & 0 & 4.647763 & 1.180231 & -1.038458 \\
\hline 35 & 6 & 0 & 4.881863 & -1.361347 & -2.149676 \\
\hline 36 & 1 & 0 & 2.938305 & -1.751204 & -1.297261 \\
\hline 37 & 6 & 0 & 5.788711 & 0.836795 & -1.753440 \\
\hline 38 & 1 & 0 & 4.552479 & 2.180716 & -0.615268 \\
\hline 39 & 6 & 0 & 5.906243 & -0.434531 & -2.309890 \\
\hline 40 & 1 & 0 & 4.970130 & -2.355875 & -2.583321 \\
\hline 41 & 1 & 0 & 6.586353 & 1.566801 & -1.881357 \\
\hline 42 & 1 & 0 & 6.799189 & -0.700899 & -2.873406 \\
\hline 43 & 6 & 0 & 1.906619 & 2.461239 & -0.221310 \\
\hline 44 & 6 & 0 & 1.732906 & 3.372707 & 0.821082 \\
\hline 45 & 6 & 0 & 1.792896 & 2.907230 & -1.543673 \\
\hline 46 & 6 & 0 & 1.438293 & 4.706083 & 0.546170 \\
\hline 47 & 1 & 0 & 1.822329 & 3.044035 & 1.856044 \\
\hline 48 & 6 & 0 & 1.501762 & 4.236141 & -1.814462 \\
\hline 49 & 1 & 0 & 1.916954 & 2.202709 & -2.366860 \\
\hline 50 & 6 & 0 & 1.318325 & 5.138782 & -0.768563 \\
\hline 51 & 1 & 0 & 1.304531 & 5.408675 & 1.367216 \\
\hline 52 & 1 & 0 & 1.405238 & 4.567042 & -2.847225 \\
\hline 53 & 1 & 0 & 1.082430 & 6.180115 & -0.982270 \\
\hline 54 & 6 & 0 & -3.284765 & -4.514993 & -2.695644 \\
\hline 55 & 1 & 0 & -4.227178 & -4.978419 & -2.393770 \\
\hline 56 & 1 & 0 & -2.513933 & -5.290701 & -2.796487 \\
\hline 57 & 1 & 0 & -3.394562 & -4.044657 & -3.680927 \\
\hline 58 & 1 & 0 & -2.523516 & 3.792001 & -3.129760 \\
\hline 59 & 6 & 0 & -3.302975 & -0.897718 & 0.470788 \\
\hline 60 & 6 & 0 & -3.875224 & -0.831160 & 1.747784 \\
\hline 61 & 6 & 0 & -3.740496 & -0.026693 & -0.536947 \\
\hline 62 & 6 & 0 & -4.867531 & 0.094649 & 2.026149 \\
\hline 63 & 1 & 0 & -3.533501 & -1.515587 & 2.522865 \\
\hline 64 & 6 & 0 & -4.733886 & 0.900231 & -0.271601 \\
\hline 65 & 1 & 0 & -3.282126 & -0.065918 & -1.524084 \\
\hline 66 & 6 & 0 & -5.277776 & 0.944109 & 1.006467 \\
\hline 67 & 1 & 0 & -5.325639 & 0.172935 & 3.007354 \\
\hline 68 & 1 & 0 & -5.081673 & 1.595473 & -1.029965 \\
\hline 69 & 7 & 0 & -6.327705 & 1.935035 & 1.293348 \\
\hline
\end{tabular}




\begin{tabular}{|c|c|c|c|c|c|}
\hline 70 & 8 & 0 & -6.665219 & 2.674300 & 0.384149 \\
\hline 71 & 8 & 0 & -6.791855 & 1.952243 & 2.421216 \\
\hline Zero-poi & ion $=$ & & \multicolumn{3}{|c|}{0.545227 (Hartree/Particle) } \\
\hline Thermal & $\mathrm{n}$ to & & \multicolumn{3}{|c|}{0.584777} \\
\hline Thermal & $\mathrm{n}$ to & & \multicolumn{3}{|c|}{0.585721} \\
\hline Thermal & $\mathrm{n}$ to & Energy= & \multicolumn{3}{|c|}{0.466935} \\
\hline Sum of $e$ & and & Energies $=$ & \multirow{2}{*}{\multicolumn{3}{|c|}{$\begin{array}{c}-2109.191842 \\
-2109.152292\end{array}$}} \\
\hline Sum of $\mathrm{e}$ & and & ergies $=$ & & & \\
\hline Sum of $e$ & & & \multicolumn{3}{|c|}{-2109.151347} \\
\hline Sum of $\mathrm{e}$ & & & \multicolumn{3}{|c|}{-2109.270134} \\
\hline
\end{tabular}

M06 /6-311++G(d,p)-SDD/SMD//M06/6-31G(d)-LANL2DZ energy in toluene solvent $=-2111.4351894$

TS6ea

\begin{tabular}{|c|c|c|c|c|c|}
\hline \multirow{2}{*}{$\begin{array}{l}\text { Center } \\
\text { Number }\end{array}$} & \multirow{2}{*}{$\begin{array}{l}\text { Atomic } \\
\text { Number }\end{array}$} & \multirow{2}{*}{$\begin{array}{l}\text { Atomic } \\
\text { Type }\end{array}$} & \multicolumn{3}{|c|}{ Coordinates (Angstroms) } \\
\hline & & & $\mathrm{X}$ & $\mathrm{Y}$ & Z \\
\hline 1 & 6 & 0 & 0.831296 & 4.867355 & -0.028502 \\
\hline 2 & 6 & 0 & 0.479110 & 4.153805 & -1.170779 \\
\hline 3 & 6 & 0 & 0.500335 & 2.760137 & -1.161873 \\
\hline 4 & 6 & 0 & 0.863591 & 2.060897 & -0.008100 \\
\hline 5 & 6 & 0 & 1.236566 & 2.783007 & 1.127309 \\
\hline 6 & 6 & 0 & 1.211066 & 4.178246 & 1.119553 \\
\hline 7 & 1 & 0 & 0.177515 & 4.682763 & -2.075014 \\
\hline 8 & 1 & 0 & 0.200336 & 2.219084 & -2.063015 \\
\hline 9 & 1 & 0 & 1.545492 & 2.257990 & 2.033129 \\
\hline 10 & 1 & 0 & 1.494732 & 4.727935 & 2.017407 \\
\hline 11 & 53 & 0 & 1.110744 & -2.748548 & 0.092589 \\
\hline 12 & 46 & 0 & 0.888569 & 0.011905 & 0.037264 \\
\hline 13 & 15 & 0 & -1.488933 & -0.042712 & 0.068171 \\
\hline 14 & 6 & 0 & 3.896254 & 0.077453 & 1.047092 \\
\hline 15 & 1 & 0 & 3.728696 & -0.140571 & 2.099352 \\
\hline 16 & 6 & 0 & 2.955370 & 0.133095 & 0.089807 \\
\hline 17 & 6 & 0 & 3.279708 & 0.409596 & -1.233635 \\
\hline 18 & 6 & 0 & 5.230935 & 0.324183 & 0.505723 \\
\hline 19 & 8 & 0 & 5.367010 & 0.579593 & -0.697359 \\
\hline 20 & 6 & 0 & -2.147443 & -1.169551 & -1.209968 \\
\hline 21 & 6 & 0 & -3.216397 & -2.035177 & -0.982202 \\
\hline 22 & 6 & 0 & -1.544722 & -1.139427 & -2.472005 \\
\hline 23 & 6 & 0 & -3.682080 & -2.853629 & -2.007685 \\
\hline 24 & 1 & 0 & -3.679479 & -2.085869 & 0.002561 \\
\hline 25 & 6 & 0 & -2.020473 & -1.945117 & -3.497641 \\
\hline 26 & 1 & 0 & -0.684684 & -0.486835 & -2.639840 \\
\hline 27 & 6 & 0 & -3.090227 & -2.806119 & -3.264574 \\
\hline 28 & 1 & 0 & -4.509183 & -3.535849 & -1.818738 \\
\hline 29 & 1 & 0 & -1.545954 & -1.914085 & -4.476915 \\
\hline 30 & 1 & 0 & -3.455089 & -3.449300 & -4.063665 \\
\hline 31 & 6 & 0 & -2.241817 & -0.563913 & 1.650194 \\
\hline 32 & 6 & 0 & -1.481060 & -1.268746 & 2.585920 \\
\hline 33 & 6 & 0 & -3.582509 & -0.272704 & 1.933859 \\
\hline 34 & 6 & 0 & -2.054953 & -1.680280 & 3.785330 \\
\hline 35 & 1 & 0 & -0.441288 & -1.509645 & 2.362648 \\
\hline 36 & 6 & 0 & -4.152319 & -0.689325 & 3.130643 \\
\hline 37 & 1 & 0 & -4.180444 & 0.288566 & 1.215019 \\
\hline 38 & 6 & 0 & -3.387470 & -1.392569 & 4.058335 \\
\hline 39 & 1 & 0 & -1.454094 & -2.230824 & 4.506895 \\
\hline 40 & 1 & 0 & -5.195429 & -0.459678 & 3.342270 \\
\hline 41 & 1 & 0 & -3.833309 & -1.715399 & 4.997941 \\
\hline 42 & 6 & 0 & -2.338179 & 1.545388 & -0.272505 \\
\hline 43 & 6 & 0 & -3.007805 & 1.798390 & -1.469839 \\
\hline 44 & 6 & 0 & -2.221560 & 2.569437 & 0.674636 \\
\hline 45 & 6 & 0 & -3.545969 & 3.059327 & -1.719286 \\
\hline 46 & 1 & 0 & -3.112933 & 1.011000 & -2.215251 \\
\hline 47 & 6 & 0 & -2.759679 & 3.823161 & 0.424965 \\
\hline 48 & 1 & 0 & -1.682613 & 2.388119 & 1.605418 \\
\hline 49 & 6 & 0 & -3.420349 & 4.072198 & -0.776830 \\
\hline 50 & 1 & 0 & -4.067509 & 3.245534 & -2.656982 \\
\hline 51 & 1 & 0 & -2.647772 & 4.613937 & 1.165011 \\
\hline 52 & 1 & 0 & -3.837559 & 5.058285 & -0.975297 \\
\hline 53 & 6 & 0 & 6.413938 & 0.262075 & 1.420158 \\
\hline 54 & 1 & 0 & 6.288145 & 0.979602 & 2.241485 \\
\hline 55 & 1 & 0 & 7.337341 & 0.480123 & 0.877786 \\
\hline 56 & 1 & 0 & 6.478918 & -0.734489 & 1.876382 \\
\hline 57 & 1 & 0 & 3.187515 & 1.455252 & -1.545881 \\
\hline 58 & 1 & 0 & 0.811702 & 5.956279 & -0.033946 \\
\hline 59 & 6 & 0 & 3.406233 & -0.620197 & -2.297385 \\
\hline 60 & 1 & 0 & 3.595949 & -1.610429 & -1.870198 \\
\hline 61 & 1 & 0 & 2.470302 & -0.674103 & -2.871709 \\
\hline 62 & 1 & 0 & 4.208653 & -0.334027 & -2.986175 \\
\hline \multicolumn{6}{|c|}{ Zero-point correction $=$} \\
\hline \multicolumn{3}{|c|}{ Thermal correction to Energy= } & \multicolumn{2}{|c|}{0.523475} & \\
\hline Thermal & rrection to $\mathrm{E}$ & alpy $=$ & \multicolumn{2}{|c|}{0.524419} & \\
\hline Thermal & rrection to $\mathrm{C}$ & bs Free Energy= & \multicolumn{2}{|c|}{0.419837} & \\
\hline Sum of $\mathrm{e}$ & tronic and $z$ & -point Energies= & & 1713.248032 & \\
\hline Sum of e & tronic and $\mathrm{t}$ & mal Energies= & & -1713.214126 & \\
\hline Sum of e & tronic and $\mathrm{t}$ & mal Enthalpies= & & -1713.213182 & \\
\hline Sum of e & tronic and $\mathrm{t}$ & mal Free Energies & & 1713.317764 & \\
\hline
\end{tabular}




\begin{tabular}{|c|c|c|c|c|c|}
\hline \multirow{2}{*}{$\begin{array}{l}\text { Center } \\
\text { Number }\end{array}$} & \multirow{2}{*}{$\begin{array}{l}\text { Atomic } \\
\text { Number }\end{array}$} & \multirow{2}{*}{$\begin{array}{l}\text { Atomic } \\
\text { Type }\end{array}$} & \multicolumn{3}{|c|}{ Coordinates (Angstroms) } \\
\hline & & & $\mathrm{X}$ & $\mathrm{Y}$ & Z \\
\hline 1 & 6 & 0 & 0.480510 & 4.853022 & 0.087799 \\
\hline 2 & 6 & 0 & 0.452731 & 4.137543 & -1.105733 \\
\hline 3 & 6 & 0 & 0.495404 & 2.743835 & -1.091865 \\
\hline 4 & 6 & 0 & 0.573485 & 2.046301 & 0.115201 \\
\hline 5 & 6 & 0 & 0.602621 & 2.771432 & 1.308543 \\
\hline 6 & 6 & 0 & 0.551682 & 4.165589 & 1.296247 \\
\hline 7 & 1 & 0 & 0.389051 & 4.664579 & -2.057901 \\
\hline 8 & 1 & 0 & 0.442649 & 2.202500 & -2.039919 \\
\hline 9 & 1 & 0 & 0.647783 & 2.246685 & 2.266599 \\
\hline 10 & 1 & 0 & 0.568601 & 4.715417 & 2.237682 \\
\hline 11 & 53 & 0 & 0.653790 & -2.764067 & 0.586105 \\
\hline 12 & 46 & 0 & 0.615537 & 0.003370 & 0.217027 \\
\hline 13 & 15 & 0 & -1.736336 & -0.021791 & -0.052728 \\
\hline 14 & 6 & 0 & 3.354702 & 0.705966 & 1.597646 \\
\hline 15 & 1 & 0 & 2.921066 & 1.285870 & 2.409827 \\
\hline 16 & 6 & 0 & 2.677613 & 0.178184 & 0.563920 \\
\hline 17 & 6 & 0 & 3.298650 & -0.569387 & -0.426840 \\
\hline 18 & 1 & 0 & 3.291139 & -1.656302 & -0.267327 \\
\hline 19 & 6 & 0 & 4.780631 & 0.389024 & 1.557123 \\
\hline 20 & 8 & 0 & 5.236424 & -0.281341 & 0.624809 \\
\hline 21 & 6 & 0 & -2.241656 & -1.352169 & -1.193779 \\
\hline 22 & 6 & 0 & -3.118895 & -2.371227 & -0.826440 \\
\hline 23 & 6 & 0 & -1.622294 & -1.398333 & -2.448079 \\
\hline 24 & 6 & 0 & -3.381421 & -3.416363 & -1.707035 \\
\hline 25 & 1 & 0 & -3.578175 & -2.366612 & 0.160984 \\
\hline 26 & 6 & 0 & -1.895740 & -2.434672 & -3.329433 \\
\hline 27 & 1 & 0 & -0.903317 & -0.623840 & -2.724552 \\
\hline 28 & 6 & 0 & -2.774749 & -3.449183 & -2.956991 \\
\hline 29 & 1 & 0 & -4.056457 & -4.215800 & -1.406590 \\
\hline 30 & 1 & 0 & -1.407807 & -2.462643 & -4.302259 \\
\hline 31 & 1 & 0 & -2.976875 & -4.272143 & -3.640390 \\
\hline 32 & 6 & 0 & -2.634170 & -0.287477 & 1.514332 \\
\hline 33 & 6 & 0 & -1.928097 & -0.629021 & 2.669231 \\
\hline 34 & 6 & 0 & -4.023988 & -0.127599 & 1.576828 \\
\hline 35 & 6 & 0 & -2.604362 & -0.816184 & 3.871735 \\
\hline 36 & 1 & 0 & -0.846540 & -0.760196 & 2.616027 \\
\hline 37 & 6 & 0 & -4.695958 & -0.320829 & 2.776969 \\
\hline 38 & 1 & 0 & -4.579434 & 0.154591 & 0.681468 \\
\hline 39 & 6 & 0 & -3.985110 & -0.664363 & 3.925283 \\
\hline 40 & 1 & 0 & -2.046860 & -1.084961 & 4.767139 \\
\hline 41 & 1 & 0 & -5.776999 & -0.198740 & 2.818958 \\
\hline 42 & 1 & 0 & -4.512331 & -0.811664 & 4.866676 \\
\hline 43 & 6 & 0 & -2.529738 & 1.497224 & -0.703888 \\
\hline 44 & 6 & 0 & -2.999722 & 1.608482 & -2.013182 \\
\hline 45 & 6 & 0 & -2.578058 & 2.617942 & 0.133853 \\
\hline 46 & 6 & 0 & -3.496515 & 2.823445 & -2.479957 \\
\hline 47 & 1 & 0 & -2.987241 & 0.743792 & -2.675096 \\
\hline 48 & 6 & 0 & -3.072803 & 3.826451 & -0.333547 \\
\hline 49 & 1 & 0 & -2.200260 & 2.551026 & 1.154207 \\
\hline 50 & 6 & 0 & -3.529723 & 3.933788 & -1.645109 \\
\hline 51 & 1 & 0 & -3.862701 & 2.896657 & -3.502899 \\
\hline 52 & 1 & 0 & -3.087728 & 4.692409 & 0.326342 \\
\hline 53 & 1 & 0 & -3.913172 & 4.883639 & -2.014633 \\
\hline 54 & 6 & 0 & 5.662752 & 0.873695 & 2.666138 \\
\hline 55 & 1 & 0 & 5.350658 & 0.409908 & 3.611477 \\
\hline 56 & 1 & 0 & 5.550699 & 1.958279 & 2.792923 \\
\hline 57 & 1 & 0 & 6.708729 & 0.626401 & 2.467931 \\
\hline 58 & 1 & 0 & 0.443121 & 5.941444 & 0.076353 \\
\hline 59 & 6 & 0 & 3.636594 & -0.120926 & -1.819560 \\
\hline 60 & 6 & 0 & 2.413562 & -0.448377 & -2.697059 \\
\hline 61 & 1 & 0 & 2.144574 & -1.512370 & -2.639513 \\
\hline 62 & 1 & 0 & 1.537776 & 0.136962 & -2.383749 \\
\hline 63 & 1 & 0 & 2.652682 & -0.202914 & -3.742111 \\
\hline 64 & 6 & 0 & 3.944667 & 1.370864 & -1.885511 \\
\hline 65 & 1 & 0 & 4.826037 & 1.615047 & -1.279097 \\
\hline 66 & 1 & 0 & 4.160338 & 1.655097 & -2.924770 \\
\hline 67 & 1 & 0 & 3.101023 & 1.980964 & -1.535000 \\
\hline 68 & 6 & 0 & 4.824905 & -0.941905 & -2.326908 \\
\hline 69 & 1 & 0 & 5.726390 & -0.738909 & -1.739706 \\
\hline 70 & 1 & 0 & 4.614255 & -2.019217 & -2.276402 \\
\hline 71 & 1 & 0 & 5.022332 & -0.685482 & -3.376637 \\
\hline
\end{tabular}

Zero-point correction $=$

Thermal correction to Energy=

0.573913 (Hartree/Particle)

Thermal correction to Enthalpy

Thermal correction to Gibbs Free Energy= Sum of electronic and zero-point Energies

Sum of electronic and thermal Energies=

Sum of electronic and thermal Enthalpies

Sum of electronic and thermal Free Energies=

0.611692

0.500857

$-1831.011586$

$-1830.973807$

$-1830.972862$

M06 /6-311++G(d,p)-SDD/SMD//M06/6-31G(d)-LANL2DZ energy in toluene solvent $=-1833.2074722$

TS6ga

\begin{tabular}{cccccc} 
Center & Atomic & Atomic & \multicolumn{3}{c}{ Coordinates (Angstroms) } \\
Number & Number & Type & X & Y & Z \\
- \hdashline-1 & 6 & 0 & -0.817800 & 4.698378 & -0.559667 \\
2 & 6 & 0 & -0.776111 & 4.058884 & 0.676001 \\
3 & 6 & 0 & -0.743438 & 2.667486 & 0.746436
\end{tabular}




\begin{tabular}{|c|c|c|c|c|c|}
\hline 4 & 6 & 0 & -0.748173 & 1.904852 & -0.419706 \\
\hline 5 & 6 & 0 & -0.803891 & 2.546396 & -1.657750 \\
\hline 6 & 6 & 0 & -0.834085 & 3.939844 & -1.726142 \\
\hline 7 & 1 & 0 & -0.759069 & 4.645154 & 1.594677 \\
\hline 8 & 1 & 0 & -0.687493 & 2.183583 & 1.722601 \\
\hline 9 & 1 & 0 & -0.807226 & 1.961152 & -2.580288 \\
\hline 10 & 1 & 0 & -0.870350 & 4.431733 & -2.698573 \\
\hline 11 & 53 & 0 & -0.509661 & -2.915728 & -0.498096 \\
\hline 12 & 46 & 0 & -0.635426 & -0.140350 & -0.378299 \\
\hline 13 & 15 & 0 & 1.702998 & 0.040709 & 0.023795 \\
\hline 14 & 6 & 0 & -3.563559 & 0.259186 & -1.651769 \\
\hline 15 & 1 & 0 & -3.345253 & 0.725032 & -2.609246 \\
\hline 16 & 6 & 0 & -2.682875 & -0.095018 & -0.704985 \\
\hline 17 & 6 & 0 & -3.113953 & -0.678977 & 0.474065 \\
\hline 18 & 1 & 0 & -3.057975 & -1.764800 & 0.594622 \\
\hline 19 & 6 & 0 & -4.931820 & -0.051222 & -1.231792 \\
\hline 20 & 8 & 0 & -5.131264 & -0.565557 & -0.123481 \\
\hline 21 & 6 & 0 & 2.185234 & -1.026105 & 1.425261 \\
\hline 22 & 6 & 0 & 3.309384 & -1.850213 & 1.403762 \\
\hline 23 & 6 & 0 & 1.362440 & -1.013067 & 2.557150 \\
\hline 24 & 6 & 0 & 3.617104 & -2.637325 & 2.509935 \\
\hline 25 & 1 & 0 & 3.940382 & -1.892832 & 0.517080 \\
\hline 26 & 6 & 0 & 1.679523 & -1.789653 & 3.663465 \\
\hline 27 & 1 & 0 & 0.458697 & -0.399980 & 2.562818 \\
\hline 28 & 6 & 0 & 2.808955 & -2.604307 & 3.640284 \\
\hline 29 & 1 & 0 & 4.492329 & -3.284288 & 2.483245 \\
\hline 30 & 1 & 0 & 1.034476 & -1.772067 & 4.540138 \\
\hline 31 & 1 & 0 & 3.052505 & -3.222350 & 4.502861 \\
\hline 32 & 6 & 0 & 2.774299 & -0.402245 & -1.386532 \\
\hline 33 & 6 & 0 & 2.231888 & -1.050367 & -2.497608 \\
\hline 34 & 6 & 0 & 4.138224 & -0.082119 & -1.373105 \\
\hline 35 & 6 & 0 & 3.044524 & -1.383447 & -3.577567 \\
\hline 36 & 1 & 0 & 1.172813 & -1.308932 & -2.503095 \\
\hline 37 & 6 & 0 & 4.946973 & -0.421725 & -2.450110 \\
\hline 38 & 1 & 0 & 4.565690 & 0.441046 & -0.517010 \\
\hline 39 & 6 & 0 & 4.399262 & -1.072364 & -3.553728 \\
\hline 40 & 1 & 0 & 2.613125 & -1.891012 & -4.438350 \\
\hline 41 & 1 & 0 & 6.006647 & -0.172671 & -2.431341 \\
\hline 42 & 1 & 0 & 5.032824 & -1.334704 & -4.399612 \\
\hline 43 & 6 & 0 & 2.319504 & 1.701603 & 0.490397 \\
\hline 44 & 6 & 0 & 2.628169 & 2.042653 & 1.807944 \\
\hline 45 & 6 & 0 & 2.379870 & 2.687194 & -0.501983 \\
\hline 46 & 6 & 0 & 2.984915 & 3.350545 & 2.128440 \\
\hline 47 & 1 & 0 & 2.590849 & 1.287696 & 2.592194 \\
\hline 48 & 6 & 0 & 2.732351 & 3.989411 & -0.179060 \\
\hline 49 & 1 & 0 & 2.134372 & 2.437199 & -1.534377 \\
\hline 50 & 6 & 0 & 3.034272 & 4.324921 & 1.139276 \\
\hline 51 & 1 & 0 & 3.227199 & 3.603959 & 3.159407 \\
\hline 52 & 1 & 0 & 2.762633 & 4.748263 & -0.959327 \\
\hline 53 & 1 & 0 & 3.306917 & 5.348161 & 1.392920 \\
\hline 54 & 6 & 0 & -6.070084 & 0.267260 & -2.145250 \\
\hline 55 & 1 & 0 & -7.019640 & -0.063213 & -1.717687 \\
\hline 56 & 1 & 0 & -5.911588 & -0.211880 & -3.119982 \\
\hline 57 & 1 & 0 & -6.103050 & 1.350054 & -2.324591 \\
\hline 58 & 1 & 0 & -0.839284 & 5.786015 & -0.613856 \\
\hline 59 & 6 & 0 & -3.300381 & 0.101549 & 1.755071 \\
\hline 60 & 9 & 0 & -2.159163 & 0.122032 & 2.457720 \\
\hline 61 & 9 & 0 & -3.662488 & 1.355148 & 1.519429 \\
\hline 62 & 9 & 0 & -4.221474 & -0.479278 & 2.513086 \\
\hline
\end{tabular}

Zero-point correction=

0.467074 (Hartree/Particle)

Thermal correction to Energy=

Thermal corection to Enthalpy=

Thermal correction to Gibbs Free Energy=

Sum of electronic and zero-point Energies

Sum of electronic and thermal Energies=

Sum of electronic and thermal Enthalpies=

0.502765

0.503709

0.395546

$-2010.912940$

$-2010.877249$

$-2010.876305$

Sum of electronic and thermal Free Energies=

$-2010.984467$

M06 /6-311++G(d,p)-SDD/SMD//M06/6-31G(d)-LANL2DZ energy in toluene solvent $=-2013.0487473$

TS6h

\begin{tabular}{rrrrrr} 
Center & Atomic & Atomic & \multicolumn{2}{c}{ Coordinates (Angstroms) } \\
Number & Number & Type & $\mathrm{X}$ & $\mathrm{Y}$ & $\mathrm{Z}$ \\
$-\mathrm{-a}$ & 6 & 0 & 0.629046 & 4.883703 & -0.276332 \\
2 & 6 & 0 & 0.149487 & 4.152057 & -1.359643 \\
3 & 6 & 0 & 0.210180 & 2.759659 & -1.347665 \\
4 & 6 & 0 & 0.746962 & 2.082555 & -0.250483 \\
5 & 6 & 0 & 1.243386 & 2.821283 & 0.824840 \\
6 & 6 & 0 & 1.174837 & 4.215206 & 0.815386 \\
7 & 1 & 0 & -0.286145 & 4.664694 & -2.217395 \\
8 & 1 & 0 & -0.201650 & 2.200172 & -2.191101 \\
9 & 1 & 0 & 1.687103 & 2.311578 & 1.682054 \\
10 & 1 & 0 & 1.556505 & 4.779684 & 1.666476 \\
11 & 53 & 0 & 1.238861 & -2.703297 & -0.316005 \\
12 & 46 & 0 & 0.855203 & 0.037381 & -0.239299 \\
13 & 15 & 0 & -1.499803 & -0.101867 & 0.104243 \\
14 & 6 & 0 & 3.926504 & 0.183034 & 0.611914 \\
15 & 6 & 0 & 2.916334 & 0.227409 & -0.271489 \\
16 & 6 & 0 & 3.095497 & 0.412774 & -1.623576 \\
17 & 1 & 0 & 3.114987 & -0.446373 & -2.296983 \\
18 & 6 & 0 & 5.200478 & 0.348645 & -0.110061
\end{tabular}




\begin{tabular}{|c|c|c|c|c|c|}
\hline 19 & 8 & 0 & 5.209854 & 0.523655 & -1.335186 \\
\hline 20 & 6 & 0 & -2.341913 & -1.268365 & -1.021033 \\
\hline 21 & 6 & 0 & -3.468203 & -1.997841 & -0.638586 \\
\hline 22 & 6 & 0 & -1.860910 & -1.378739 & -2.328904 \\
\hline 23 & 6 & 0 & -4.107388 & -2.825065 & -1.556588 \\
\hline 24 & 1 & 0 & -3.842505 & -1.930692 & 0.382499 \\
\hline 25 & 6 & 0 & -2.507848 & -2.196467 & -3.247086 \\
\hline 26 & 1 & 0 & -0.964049 & -0.827796 & -2.618875 \\
\hline 27 & 6 & 0 & -3.630963 & -2.922392 & -2.859648 \\
\hline 28 & 1 & 0 & -4.979819 & -3.399720 & -1.249872 \\
\hline 29 & 1 & 0 & -2.123714 & -2.281047 & -4.262012 \\
\hline 30 & 1 & 0 & -4.130820 & -3.573661 & -3.574778 \\
\hline 31 & 6 & 0 & -1.952304 & -0.607218 & 1.803333 \\
\hline 32 & 6 & 0 & -1.040512 & -1.329619 & 2.577728 \\
\hline 33 & 6 & 0 & -3.206342 & -0.289167 & 2.340755 \\
\hline 34 & 6 & 0 & -1.381004 & -1.731039 & 3.866069 \\
\hline 35 & 1 & 0 & -0.066807 & -1.590911 & 2.161113 \\
\hline 36 & 6 & 0 & -3.542286 & -0.693044 & 3.627331 \\
\hline 37 & 1 & 0 & -3.918270 & 0.289280 & 1.751168 \\
\hline 38 & 6 & 0 & -2.628620 & -1.414117 & 4.391802 \\
\hline 39 & 1 & 0 & -0.663978 & -2.295457 & 4.459530 \\
\hline 40 & 1 & 0 & -4.519284 & -0.439803 & 4.035738 \\
\hline 41 & 1 & 0 & -2.890772 & -1.726410 & 5.401499 \\
\hline 42 & 6 & 0 & -2.432095 & 1.457045 & -0.127392 \\
\hline 43 & 6 & 0 & -3.202722 & 1.691212 & -1.267170 \\
\hline 44 & 6 & 0 & -2.260333 & 2.487547 & 0.804208 \\
\hline 45 & 6 & 0 & -3.789490 & 2.937731 & -1.473129 \\
\hline 46 & 1 & 0 & -3.345450 & 0.899855 & -2.002583 \\
\hline 47 & 6 & 0 & -2.849864 & 3.726291 & 0.599133 \\
\hline 48 & 1 & 0 & -1.644621 & 2.322895 & 1.688857 \\
\hline 49 & 6 & 0 & -3.613371 & 3.955716 & -0.543860 \\
\hline 50 & 1 & 0 & -4.389084 & 3.109552 & -2.365686 \\
\hline 51 & 1 & 0 & -2.699751 & 4.521048 & 1.328053 \\
\hline 52 & 1 & 0 & -4.069880 & 4.930655 & -0.707936 \\
\hline 53 & 6 & 0 & 6.488801 & 0.293255 & 0.651519 \\
\hline 54 & 1 & 0 & 6.574125 & -0.665139 & 1.180764 \\
\hline 55 & 1 & 0 & 6.512136 & 1.080272 & 1.416950 \\
\hline 56 & 1 & 0 & 7.337402 & 0.415575 & -0.025785 \\
\hline 57 & 1 & 0 & 2.969656 & 1.399719 & -2.072569 \\
\hline 58 & 1 & 0 & 0.577830 & 5.971608 & -0.283818 \\
\hline 59 & 6 & 0 & 3.836943 & -0.056181 & 2.082627 \\
\hline 60 & 1 & 0 & 4.331624 & -0.993930 & 2.373666 \\
\hline 61 & 1 & 0 & 4.295188 & 0.753404 & 2.669017 \\
\hline 62 & 1 & 0 & 2.785593 & -0.140238 & 2.381868 \\
\hline
\end{tabular}

Zero-point correction=

0.488799 (Hartree/Particle)

Thermal correction to Energy=

to Enthalpy=

Thermal correction to Gibbs Free Energy=

Sum of electronic and zero-point Energies=

Sum of electronic and thermal Energies=

Sum of electronic and thermal Enthalpies=

0.522036
0.522981

0.522036
0.522981
0.420671

$-1713.244744$

$-1713.211507$

Sum of electronic and thermal Free Energies

$-1713.210563$

M06 /6-311++G(d,p)-SDD/SMD//M06/6-31G(d)-LANL2DZ energy in toluene solvent = -1715.321172

TS6ia

\begin{tabular}{rrrrrr} 
Center & Atomic & Atomic & \multicolumn{2}{c}{ Coordinates (Angstroms) } \\
Number & Number & Type & X & Y & $Z$ \\
--1 & 6 & 0 & 1.282643 & 4.326183 & -1.431833 \\
\hline 2 & 6 & 0 & 0.589115 & 3.587770 & -2.386829 \\
3 & 6 & 0 & 0.303932 & 2.241270 & -2.161322 \\
4 & 6 & 0 & 0.707419 & 1.624414 & -0.976998 \\
5 & 6 & 0 & 1.400196 & 2.367399 & -0.018868 \\
6 & 6 & 0 & 1.685577 & 3.713746 & -0.247898 \\
7 & 1 & 0 & 0.263805 & 4.060622 & -3.313563 \\
8 & 1 & 0 & -0.265421 & 1.680937 & -2.907143 \\
9 & 1 & 0 & 1.729198 & 1.901031 & 0.913690 \\
10 & 1 & 0 & 2.232420 & 4.280700 & 0.506286 \\
11 & 53 & 0 & 0.067236 & -3.056397 & -0.062020 \\
12 & 46 & 0 & 0.345846 & -0.358339 & -0.629422 \\
13 & 15 & 0 & -1.824507 & 0.220534 & 0.140539 \\
14 & 6 & 0 & 3.538747 & -0.467036 & -0.653452 \\
15 & 6 & 0 & 2.318063 & -0.631154 & -1.197940 \\
16 & 6 & 0 & 2.115234 & -1.031400 & -2.497631 \\
17 & 1 & 0 & 1.876970 & -2.070829 & -2.731333 \\
18 & 6 & 0 & 4.570541 & -0.826925 & -1.647485 \\
19 & 8 & 0 & 4.233349 & -1.176311 & -2.785273 \\
20 & 6 & 0 & -3.106629 & -0.978554 & -0.356316 \\
21 & 6 & 0 & -4.040307 & -1.503948 & 0.535482 \\
22 & 6 & 0 & -3.114877 & -1.400578 & -1.690202 \\
23 & 6 & 0 & -4.980939 & -2.429786 & 0.092698 \\
24 & 1 & 0 & -4.024524 & -1.205545 & 1.582829 \\
25 & 6 & 0 & -4.064495 & -2.311130 & -2.132831 \\
26 & 1 & 0 & -2.355463 & -1.023245 & -2.378675 \\
27 & 6 & 0 & -4.998448 & -2.828739 & -1.238707 \\
28 & 1 & 0 & -5.700344 & -2.844696 & 0.796756 \\
29 & 1 & 0 & -4.062825 & -2.634094 & -3.172275 \\
30 & 1 & 0 & -5.733603 & -3.555279 & -1.580885 \\
31 & 6 & 0 & -1.881194 & 0.336168 & 1.960806 \\
32 & 6 & 0 & -0.842796 & -0.219783 & 2.711289 \\
33 & 6 & 0 & -2.939031 & 0.977042 & 2.617763
\end{tabular}




\begin{tabular}{|c|c|c|c|c|c|}
\hline 34 & 6 & 0 & -0.864368 & -0.141426 & 4.100508 \\
\hline 35 & 1 & 0 & -0.023773 & -0.726112 & 2.198021 \\
\hline 36 & 6 & 0 & -2.958371 & 1.050816 & 4.004833 \\
\hline 37 & 1 & 0 & -3.745122 & 1.427897 & 2.037829 \\
\hline 38 & 6 & 0 & -1.919883 & 0.491921 & 4.746741 \\
\hline 39 & 1 & 0 & -0.052479 & -0.580246 & 4.677892 \\
\hline 40 & 1 & 0 & -3.783885 & 1.550314 & 4.509289 \\
\hline 41 & 1 & 0 & -1.934937 & 0.554261 & 5.833688 \\
\hline 42 & 6 & 0 & -2.480558 & 1.842580 & -0.403455 \\
\hline 43 & 6 & 0 & -3.400948 & 1.954127 & -1.446913 \\
\hline 44 & 6 & 0 & -1.952470 & 3.006933 & 0.166421 \\
\hline 45 & 6 & 0 & -3.782846 & 3.208642 & -1.915340 \\
\hline 46 & 1 & 0 & -3.832498 & 1.059403 & -1.894004 \\
\hline 47 & 6 & 0 & -2.335386 & 4.255746 & -0.302029 \\
\hline 48 & 1 & 0 & -1.220852 & 2.935068 & 0.971052 \\
\hline 49 & 6 & 0 & -3.250035 & 4.359856 & -1.347486 \\
\hline 50 & 1 & 0 & -4.505614 & 3.282728 & -2.726467 \\
\hline 51 & 1 & 0 & -1.903748 & 5.151437 & 0.142160 \\
\hline 52 & 1 & 0 & -3.547414 & 5.339554 & -1.718180 \\
\hline 53 & 6 & 0 & 6.024215 & -0.784735 & -1.287656 \\
\hline 54 & 1 & 0 & 6.188569 & -1.044097 & -0.236077 \\
\hline 55 & 1 & 0 & 6.407363 & 0.234038 & -1.436439 \\
\hline 56 & 1 & 0 & 6.586091 & -1.455224 & -1.943896 \\
\hline 57 & 1 & 0 & 1.943893 & -0.288137 & -3.278821 \\
\hline 58 & 1 & 0 & 1.507818 & 5.376997 & -1.610046 \\
\hline 59 & 6 & 0 & 3.796354 & -0.005897 & 0.722575 \\
\hline 60 & 6 & 0 & 4.614680 & 1.098023 & 0.981173 \\
\hline 61 & 6 & 0 & 3.140059 & -0.627188 & 1.790931 \\
\hline 62 & 6 & 0 & 4.767259 & 1.577862 & 2.277484 \\
\hline 63 & 1 & 0 & 5.099190 & 1.614364 & 0.151699 \\
\hline 64 & 6 & 0 & 3.289532 & -0.143277 & 3.085772 \\
\hline 65 & 1 & 0 & 2.507283 & -1.493118 & 1.587489 \\
\hline 66 & 6 & 0 & 4.102117 & 0.960388 & 3.332781 \\
\hline 67 & 1 & 0 & 5.397425 & 2.446342 & 2.462698 \\
\hline 68 & 1 & 0 & 2.771833 & -0.636319 & 3.907859 \\
\hline 69 & 1 & 0 & 4.216391 & 1.340377 & 4.346724 \\
\hline \multicolumn{6}{|c|}{0.542003 (Hartree/Particle) } \\
\hline Thermal & rrection to $\mathrm{El}$ & $\mathrm{rgy}=$ & & 578945 & 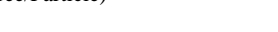 \\
\hline Thermal & rrection to $\mathrm{El}$ & halpy= & & 579889 & \\
\hline Thermal & rrection to $\mathrm{G}$ & bs Free Energy= & & 69136 & \\
\hline Sum of el & tronic and ze & -point Energies= & & 1904.778893 & \\
\hline Sum of el & tronic and th & mal Energies= & & -1904.741950 & \\
\hline Sum of el & tronic and th & mal Enthalpies= & & 1904.741006 & \\
\hline Sum of el & tronic and th & mal Free Energies= & & 1904.851760 & \\
\hline M06 /6-31 & $+\mathrm{G}(\mathrm{d}, \mathrm{p})-\mathrm{SD}$ & /SMD//M06/6-31G(c & (d)-LANL2 & Z energy in $t$ & luene solvent $=-1906.960721$ \\
\hline TS6ja & & & & & \\
\hline Center & Atomic & Atomic & Coor & linates (Angstı & oms) \\
\hline Number & Number & Type & $\mathrm{X}$ & $\mathrm{Y}$ & $\mathrm{Z}$ \\
\hline 1 & 6 & 0 & 0.158081 & 4.905656 & -0.525066 \\
\hline 2 & 6 & 0 & -0.387341 & 4.145226 & -1.555660 \\
\hline 3 & 6 & 0 & -0.278190 & 2.755711 & -1.535240 \\
\hline 4 & 6 & 0 & 0.369937 & 2.112832 & -0.478802 \\
\hline 5 & 6 & 0 & 0.929221 & 2.878957 & 0.544665 \\
\hline 6 & 6 & 0 & 0.816812 & 4.269971 & 0.523005 \\
\hline 7 & 1 & 0 & -0.912870 & 4.632750 & -2.376894 \\
\hline 8 & 1 & 0 & -0.737555 & 2.173582 & -2.337794 \\
\hline 9 & 1 & 0 & 1.450183 & 2.395679 & 1.372161 \\
\hline 10 & 1 & 0 & 1.251734 & 4.856969 & 1.332052 \\
\hline 11 & 53 & 0 & 0.911162 & -2.671561 & -0.542730 \\
\hline 12 & 46 & 0 & 0.531585 & 0.069048 & -0.452429 \\
\hline 13 & 15 & 0 & -1.760795 & -0.099650 & 0.148773 \\
\hline 14 & 6 & 0 & 3.695879 & 0.255349 & -0.058431 \\
\hline 15 & 6 & 0 & 2.553789 & 0.255699 & -0.765081 \\
\hline 16 & 6 & 0 & 2.572261 & 0.376414 & -2.139288 \\
\hline 17 & 1 & 0 & 2.485470 & -0.513033 & -2.765142 \\
\hline 18 & 6 & 0 & 4.867611 & 0.358147 & -0.940778 \\
\hline 19 & 8 & 0 & 4.682273 & 0.461917 & -2.159487 \\
\hline 20 & 6 & 0 & -2.653360 & -1.323855 & -0.868778 \\
\hline 21 & 6 & 0 & -3.551045 & -2.246546 & -0.333490 \\
\hline 22 & 6 & 0 & -2.398637 & -1.329101 & -2.244169 \\
\hline 23 & 6 & 0 & -4.190705 & -3.159637 & -1.167146 \\
\hline 24 & 1 & 0 & -3.742800 & -2.267060 & 0.738486 \\
\hline 25 & 6 & 0 & -3.048612 & -2.231228 & -3.075310 \\
\hline 26 & 1 & 0 & -1.667592 & -0.631067 & -2.657869 \\
\hline 27 & 6 & 0 & -3.943433 & -3.151586 & -2.535123 \\
\hline 28 & 1 & 0 & -4.881892 & -3.885187 & -0.741562 \\
\hline 29 & 1 & 0 & -2.840999 & -2.230681 & -4.143820 \\
\hline 30 & 1 & 0 & -4.441053 & -3.871069 & -3.183154 \\
\hline 31 & 6 & 0 & -1.982171 & -0.588720 & 1.892996 \\
\hline 32 & 6 & 0 & -0.910595 & -1.145647 & 2.595147 \\
\hline 33 & 6 & 0 & -3.214924 & -0.417338 & 2.536139 \\
\hline 34 & 6 & 0 & -1.073808 & -1.539138 & 3.920087 \\
\hline 35 & 1 & 0 & 0.050286 & -1.280404 & 2.096815 \\
\hline 36 & 6 & 0 & -3.373771 & -0.815091 & 3.857590 \\
\hline 37 & 1 & 0 & -4.048887 & 0.037224 & 1.999846 \\
\hline 38 & 6 & 0 & -2.303157 & -1.377837 & 4.549269 \\
\hline 39 & 1 & 0 & -0.234400 & -1.975161 & 4.458283 \\
\hline 40 & 1 & 0 & -4.335291 & -0.682022 & 4.350815 \\
\hline 41 & 1 & 0 & -2.429185 & -1.686946 & 5.585751 \\
\hline
\end{tabular}




\begin{tabular}{rrrrrr}
42 & 6 & 0 & -2.763379 & 1.423951 & 0.004563 \\
43 & 6 & 0 & -3.672254 & 1.617479 & -1.036631 \\
44 & 6 & 0 & -2.516124 & 2.471554 & 0.899415 \\
45 & 6 & 0 & -4.315179 & 2.843791 & -1.187142 \\
46 & 1 & 0 & -3.880371 & 0.810114 & -1.738023 \\
47 & 6 & 0 & -3.160156 & 3.690921 & 0.748515 \\
48 & 1 & 0 & -1.794426 & 2.338917 & 1.706053 \\
49 & 6 & 0 & -4.058458 & 3.881667 & -0.299641 \\
50 & 1 & 0 & -5.024008 & 2.983209 & -2.001899 \\
51 & 1 & 0 & -2.950833 & 4.500240 & 1.445935 \\
52 & 1 & 0 & -4.557413 & 4.841734 & -0.422221 \\
53 & 6 & 0 & 6.251451 & 0.349573 & -0.377609 \\
54 & 1 & 0 & 6.358064 & -0.438803 & 0.375854 \\
55 & 1 & 0 & 6.435832 & 1.303567 & 0.132841 \\
56 & 1 & 0 & 6.983494 & 0.220090 & -1.177959 \\
57 & 1 & 0 & 2.404035 & 1.346790 & -2.609196 \\
58 & 1 & 0 & 0.069864 & 5.991093 & -0.539322 \\
59 & 6 & 0 & 3.800457 & 0.091181 & 1.417594 \\
60 & 9 & 0 & 4.531852 & 1.077658 & 1.967963 \\
61 & 9 & 0 & 4.404736 & -1.058913 & 1.748813 \\
62 & 9 & 0 & 2.605487 & 0.102954 & 2.008460 \\
------------------------------------------------------ & & \\
\hline
\end{tabular}
0.503554

Thermal correction to Energy=

Thermalcorection to Enthalpy=

Thermal correction to Gibbs Free Energy=

Sum of electronic and zero-point Energies=

Sum of electronic and thermal Energies=

0.50355

Sum of electronic and thermal Enthalpies=

0.396713

$-2010.920507$

$-2010.885112$

$-2010.884168$

Sum of electronic and thermal Free Energies=

M06 /6-311++G(d,p)-SDD/SMD//M06/6-31G(d)-LANL2DZ energy in toluene solvent $=-2013.0582747$

\section{TS6k}

\begin{tabular}{|c|c|c|c|c|c|}
\hline \multirow{2}{*}{$\begin{array}{l}\text { Center } \\
\text { Number }\end{array}$} & \multirow{2}{*}{$\begin{array}{l}\text { Atomic } \\
\text { Number }\end{array}$} & \multirow{2}{*}{$\begin{array}{l}\text { Atomic } \\
\text { Type }\end{array}$} & \multicolumn{3}{|c|}{ Coordinates (Angstroms) } \\
\hline & & & $\mathrm{X}$ & $\mathrm{Y}$ & \\
\hline 1 & 6 & 0 & -0.143203 & 4.785066 & -0.338712 \\
\hline 2 & 6 & 0 & -0.676352 & 4.086086 & -1.417884 \\
\hline 3 & 6 & 0 & -0.709634 & 2.692377 & -1.403703 \\
\hline 4 & 6 & 0 & -0.216588 & 1.983977 & -0.306662 \\
\hline 5 & 6 & 0 & 0.330183 & 2.688695 & 0.766569 \\
\hline 6 & 6 & 0 & 0.359967 & 4.083384 & 0.753013 \\
\hline 7 & 1 & 0 & -1.079476 & 4.625926 & -2.274815 \\
\hline 8 & 1 & 0 & -1.157335 & 2.160230 & -2.246849 \\
\hline 9 & 1 & 0 & 0.737352 & 2.151235 & 1.625229 \\
\hline 10 & 1 & 0 & 0.785906 & 4.621861 & 1.599881 \\
\hline 11 & 53 & 0 & -0.151884 & -2.827707 & -0.243675 \\
\hline 12 & 46 & 0 & -0.264370 & -0.062459 & -0.259143 \\
\hline 13 & 15 & 0 & -2.604465 & -0.003754 & 0.202828 \\
\hline 14 & 6 & 0 & 2.931758 & 0.030538 & 0.160357 \\
\hline 15 & 1 & 0 & 3.005847 & 0.048352 & 1.242346 \\
\hline 16 & 6 & 0 & 1.790131 & -0.008174 & -0.536699 \\
\hline 17 & 6 & 0 & 1.744614 & -0.033234 & -1.913175 \\
\hline 18 & 1 & 0 & 1.625132 & -0.979612 & -2.443105 \\
\hline 19 & 6 & 0 & 4.096343 & 0.023187 & -0.740543 \\
\hline 20 & 8 & 0 & 3.904611 & 0.005265 & -1.969310 \\
\hline 21 & 6 & 0 & -3.562942 & -1.122740 & -0.876999 \\
\hline 22 & 6 & 0 & -4.668439 & -1.845628 & -0.429316 \\
\hline 23 & 6 & 0 & -3.174579 & -1.218440 & -2.217019 \\
\hline 24 & 6 & 0 & -5.381519 & -2.647911 & -1.315352 \\
\hline 25 & 1 & 0 & -4.968554 & -1.795891 & 0.616779 \\
\hline 26 & 6 & 0 & -3.895340 & -2.009518 & -3.102140 \\
\hline 27 & 1 & 0 & -2.290219 & -0.675836 & -2.557995 \\
\hline 28 & 6 & 0 & -4.999834 & -2.726972 & -2.650011 \\
\hline 29 & 1 & 0 & -6.237574 & -3.217695 & -0.957787 \\
\hline 30 & 1 & 0 & -3.584751 & -2.079669 & -4.143007 \\
\hline 31 & 1 & 0 & -5.558958 & -3.357517 & -3.339347 \\
\hline 32 & 6 & 0 & -3.032839 & -0.441898 & 1.924247 \\
\hline 33 & 6 & 0 & -2.120320 & -1.159680 & 2.701338 \\
\hline 34 & 6 & 0 & -4.263418 & -0.067769 & 2.479874 \\
\hline 35 & 6 & 0 & -2.436759 & -1.502375 & 4.012665 \\
\hline 36 & 1 & 0 & -1.165833 & -1.462614 & 2.269004 \\
\hline 37 & 6 & 0 & -4.575717 & -0.413748 & 3.788909 \\
\hline 38 & 1 & 0 & -4.974289 & 0.508099 & 1.886161 \\
\hline 39 & 6 & 0 & -3.661055 & -1.130628 & 4.556754 \\
\hline 40 & 1 & 0 & -1.719624 & -2.063351 & 4.609148 \\
\hline 41 & 1 & 0 & -5.533913 & -0.117552 & 4.212693 \\
\hline 42 & 1 & 0 & -3.904601 & -1.396805 & 5.584244 \\
\hline 43 & 6 & 0 & -3.424313 & 1.617854 & -0.028321 \\
\hline 44 & 6 & 0 & -4.210932 & 1.896685 & -1.146971 \\
\hline 45 & 6 & 0 & -3.150924 & 2.643123 & 0.884898 \\
\hline 46 & 6 & 0 & -4.707716 & 3.181529 & -1.353671 \\
\hline 47 & 1 & 0 & -4.438363 & 1.109645 & -1.865462 \\
\hline 48 & 6 & 0 & -3.647736 & 3.921503 & 0.676952 \\
\hline 49 & 1 & 0 & -2.535108 & 2.440764 & 1.761465 \\
\hline 50 & 6 & 0 & -4.424214 & 4.195632 & -0.447018 \\
\hline 51 & 1 & 0 & -5.321427 & 3.386736 & -2.229497 \\
\hline 52 & 1 & 0 & -3.420678 & 4.710423 & 1.392113 \\
\hline 53 & 1 & 0 & -4.810120 & 5.200378 & -0.612027 \\
\hline 54 & 1 & 0 & 1.600356 & 0.888318 & -2.479363 \\
\hline 55 & 1 & 0 & -0.118388 & 5.873879 & -0.349297 \\
\hline 56 & 6 & 0 & 5.469690 & 0.037668 & -0.214930 \\
\hline
\end{tabular}




\begin{tabular}{|c|c|c|c|c|c|}
\hline 57 & 6 & 0 & 5.763222 & 0.025900 & 1.157782 \\
\hline 58 & 6 & 0 & 6.533377 & 0.058985 & -1.122893 \\
\hline 59 & 6 & 0 & 7.070064 & 0.036782 & 1.600248 \\
\hline 60 & 1 & 0 & 4.963994 & 0.001815 & 1.895044 \\
\hline 61 & 6 & 0 & 7.850651 & 0.070561 & -0.693474 \\
\hline 62 & 1 & 0 & 6.300888 & 0.065371 & -2.185684 \\
\hline 63 & 6 & 0 & 8.124574 & 0.059478 & 0.678379 \\
\hline 64 & 1 & 0 & 7.314266 & 0.025225 & 2.660146 \\
\hline 65 & 1 & 0 & 8.654020 & 0.087140 & -1.425263 \\
\hline 66 & 8 & 0 & 9.363252 & 0.068488 & 1.208189 \\
\hline 67 & 6 & 0 & 10.464411 & 0.077807 & 0.329335 \\
\hline 68 & 1 & 0 & 10.466992 & 0.977537 & -0.303176 \\
\hline 69 & 1 & 0 & 11.358007 & 0.078025 & 0.957912 \\
\hline 70 & 1 & 0 & 10.473712 & -0.815142 & -0.312544 \\
\hline
\end{tabular}

Zero-point correction=

0.547294 (Hartree/Particle)

Thermal correction to Energy=

to Enthalpy=

Thermal correction to Gibbs Free Energy=

Sum of electronic and zero-point Energies

Sum of electronic and thermal Energies=

0.585302

Sum of electronic and thermal Enthalpies=

0.470967

$-1979.948456$

$-1979.910447$

$-1979.909503$

Sum of electronic and thermal Free Energies=

M06 /6-311++G(d,p)-SDD/SMD//M06/6-31G(d)-LANL2DZ energy in toluene solvent = -1982.156018

\section{TS6la}

\begin{tabular}{|c|c|c|c|c|c|}
\hline \multirow{2}{*}{$\begin{array}{l}\text { Center } \\
\text { Number }\end{array}$} & \multirow{2}{*}{$\begin{array}{l}\text { Atomic } \\
\text { Number }\end{array}$} & \multirow{2}{*}{$\begin{array}{l}\text { Atomic } \\
\text { Type }\end{array}$} & \multicolumn{3}{|c|}{ Coordinates (Angstroms) } \\
\hline & & & $\mathrm{X}$ & $\mathrm{Y}$ & \\
\hline 1 & 6 & 0 & -0.421672 & 4.834606 & -0.229311 \\
\hline 2 & 6 & 0 & -1.001784 & 4.153388 & -1.295814 \\
\hline 3 & 6 & 0 & -1.008440 & 2.759519 & -1.317592 \\
\hline 4 & 6 & 0 & -0.440739 & 2.033484 & -0.269348 \\
\hline 5 & 6 & 0 & 0.150520 & 2.719915 & 0.791593 \\
\hline 6 & 6 & 0 & 0.152773 & 4.115254 & 0.814308 \\
\hline 7 & 1 & 0 & -1.462032 & 4.706798 & -2.114319 \\
\hline 8 & 1 & 0 & -1.492933 & 2.240200 & -2.148063 \\
\hline 9 & 1 & 0 & 0.614815 & 2.168621 & 1.611540 \\
\hline 10 & 1 & 0 & 0.614109 & 4.639941 & 1.651139 \\
\hline 11 & 53 & 0 & -0.203334 & -2.770264 & -0.408765 \\
\hline 12 & 46 & 0 & -0.425797 & -0.014983 & -0.295969 \\
\hline 13 & 15 & 0 & -2.748244 & -0.046130 & 0.243563 \\
\hline 14 & 6 & 0 & 2.772821 & 0.061901 & 0.031242 \\
\hline 15 & 1 & 0 & 2.884512 & 0.001931 & 1.108694 \\
\hline 16 & 6 & 0 & 1.606086 & 0.081139 & -0.638197 \\
\hline 17 & 6 & 0 & 1.575396 & 0.183013 & -2.015911 \\
\hline 18 & 1 & 0 & 1.439017 & -0.707599 & -2.631758 \\
\hline 19 & 6 & 0 & 3.894448 & 0.129142 & -0.898032 \\
\hline 20 & 8 & 0 & 3.674077 & 0.230038 & -2.118997 \\
\hline 21 & 6 & 0 & -3.712015 & -1.143884 & -0.852513 \\
\hline 22 & 6 & 0 & -4.805215 & -1.887338 & -0.407433 \\
\hline 23 & 6 & 0 & -3.352822 & -1.188784 & -2.202958 \\
\hline 24 & 6 & 0 & -5.533692 & -2.660824 & -1.306117 \\
\hline 25 & 1 & 0 & -5.084352 & -1.875610 & 0.645559 \\
\hline 26 & 6 & 0 & -4.088565 & -1.952109 & -3.100302 \\
\hline 27 & 1 & 0 & -2.478919 & -0.629012 & -2.542859 \\
\hline 28 & 6 & 0 & -5.179954 & -2.690660 & -2.650643 \\
\hline 29 & 1 & 0 & -6.380054 & -3.246506 & -0.951311 \\
\hline 30 & 1 & 0 & -3.800408 & -1.983187 & -4.149442 \\
\hline 31 & 1 & 0 & -5.751128 & -3.299014 & -3.349896 \\
\hline 32 & 6 & 0 & -3.093993 & -0.573405 & 1.958422 \\
\hline 33 & 6 & 0 & -2.126154 & -1.284558 & 2.672106 \\
\hline 34 & 6 & 0 & -4.316460 & -0.271421 & 2.572663 \\
\hline 35 & 6 & 0 & -2.380216 & -1.692177 & 3.978419 \\
\hline 36 & 1 & 0 & -1.177289 & -1.530441 & 2.193731 \\
\hline 37 & 6 & 0 & -4.567166 & -0.683869 & 3.875624 \\
\hline 38 & 1 & 0 & -5.071534 & 0.299074 & 2.030987 \\
\hline 39 & 6 & 0 & -3.597538 & -1.393495 & 4.580017 \\
\hline 40 & 1 & 0 & -1.619458 & -2.246013 & 4.525448 \\
\hline 41 & 1 & 0 & -5.520380 & -0.445805 & 4.344746 \\
\hline 42 & 1 & 0 & -3.792803 & -1.711336 & 5.603011 \\
\hline 43 & 6 & 0 & -3.621332 & 1.557942 & 0.116816 \\
\hline 44 & 6 & 0 & -4.468180 & 1.857588 & -0.951307 \\
\hline 45 & 6 & 0 & -3.335155 & 2.551099 & 1.060790 \\
\hline 46 & 6 & 0 & -5.013606 & 3.133165 & -1.077422 \\
\hline 47 & 1 & 0 & -4.703827 & 1.094600 & -1.692691 \\
\hline 48 & 6 & 0 & -3.883823 & 3.819029 & 0.935241 \\
\hline 49 & 1 & 0 & -2.664601 & 2.333725 & 1.892814 \\
\hline 50 & 6 & 0 & -4.721128 & 4.114959 & -0.138563 \\
\hline 51 & 1 & 0 & -5.673067 & 3.355837 & -1.914810 \\
\hline 52 & 1 & 0 & -3.648210 & 4.583950 & 1.673243 \\
\hline 53 & 1 & 0 & -5.146060 & 5.112271 & -0.240800 \\
\hline 54 & 1 & 0 & 1.420156 & 1.153437 & -2.490847 \\
\hline 55 & 1 & 0 & -0.417656 & 5.923489 & -0.211626 \\
\hline 56 & 6 & 0 & 5.297356 & 0.090147 & -0.420971 \\
\hline 57 & 6 & 0 & 5.619387 & -0.324981 & 0.875022 \\
\hline 58 & 6 & 0 & 6.315925 & 0.464879 & -1.305095 \\
\hline 59 & 6 & 0 & 6.941827 & -0.359191 & 1.291869 \\
\hline 60 & 1 & 0 & 4.840087 & -0.647494 & 1.561184 \\
\hline 61 & 6 & 0 & 7.639837 & 0.444411 & -0.898386 \\
\hline 62 & 1 & 0 & 6.045268 & 0.771007 & -2.312797 \\
\hline 63 & 6 & 0 & 7.926898 & 0.033098 & 0.396909 \\
\hline
\end{tabular}




$\begin{array}{rrrrrr}64 & 1 & 0 & 7.220599 & -0.683097 & 2.289994 \\ 65 & 1 & 0 & 8.451015 & 0.736230 & -1.558811 \\ 66 & 7 & 0 & 9.332805 & 0.008679 & 0.836201 \\ 67 & 8 & 0 & 9.555970 & -0.339529 & 1.983019 \\ 68 & 8 & 0 & 10.178799 & 0.339802 & 0.023412 \\ -------------------------------------------- & & \end{array}$

$\begin{array}{lc}\text { Zero-point correction= } & 0.517138 \text { (Hartree/Particle) } \\ \text { Thermal correction to Energy= } & 0.555183 \\ \text { Thermal correction to Enthalpy= } & 0.556127 \\ \text { Thermal correction to Gibbs Free Energy= } & 0.439699 \\ \text { Sum of electronic and zero-point Energies }= & -2069.923467 \\ \text { Sum of electronic and thermal Energies }= & -2069.885422 \\ \text { Sum of electronic and thermal Enthalpies }= & -2069.884478 \\ \text { Sum of electronic and thermal Free Energies }= & -2070.000907 \\ \text { M06 /6-311++G(d,p)-SDD/SMD/M06/6-31G(d)-LANL2DZ energy in toluene solvent }=-2072.1270901\end{array}$

M06 /6-311++G(d,p)-SDD/SMD//M06/6-31G(d)-LANL2DZ energy in toluene solvent = -2072.1270901

TS6ma

\begin{tabular}{|c|c|c|c|c|c|}
\hline \multirow{2}{*}{$\begin{array}{l}\text { Center } \\
\text { Number }\end{array}$} & \multirow{2}{*}{$\begin{array}{l}\text { Atomic } \\
\text { Number }\end{array}$} & \multirow{2}{*}{$\begin{array}{l}\text { Atomic } \\
\text { Type }\end{array}$} & \multicolumn{3}{|c|}{ Coordinates (Angstroms) } \\
\hline & & & $\mathrm{X}$ & $\mathrm{Y}$ & $\mathrm{Z}$ \\
\hline 1 & 6 & 0 & 0.609843 & 4.796229 & -0.276311 \\
\hline 2 & 6 & 0 & 0.141895 & 4.079578 & -1.374023 \\
\hline 3 & 6 & 0 & 0.105640 & 2.686386 & -1.338642 \\
\hline 4 & 6 & 0 & 0.528240 & 1.996721 & -0.200749 \\
\hline 5 & 6 & 0 & 1.014450 & 2.718589 & 0.890166 \\
\hline 6 & 6 & 0 & 1.047910 & 4.112966 & 0.854339 \\
\hline 7 & 1 & 0 & -0.208071 & 4.605373 & -2.262372 \\
\hline 8 & 1 & 0 & -0.292589 & 2.141431 & -2.198268 \\
\hline 9 & 1 & 0 & 1.365744 & 2.197129 & 1.782610 \\
\hline 10 & 1 & 0 & 1.422323 & 4.665558 & 1.716147 \\
\hline 11 & 53 & 0 & 0.622599 & -2.814218 & -0.199442 \\
\hline 12 & 46 & 0 & 0.468550 & -0.052128 & -0.144537 \\
\hline 13 & 15 & 0 & -1.902199 & 0.001769 & 0.125500 \\
\hline 14 & 6 & 0 & 3.595908 & 0.063188 & 0.546735 \\
\hline 15 & 1 & 0 & 3.611158 & 0.115232 & 1.630696 \\
\hline 16 & 6 & 0 & 2.512713 & -0.001307 & -0.264363 \\
\hline 17 & 6 & 0 & 2.718907 & -0.061845 & -1.638258 \\
\hline 18 & 1 & 0 & 2.632010 & -1.018314 & -2.157730 \\
\hline 19 & 6 & 0 & 4.784951 & 0.029249 & -0.257422 \\
\hline 20 & 8 & 0 & 4.738052 & -0.036693 & -1.494015 \\
\hline 21 & 6 & 0 & -2.762211 & -1.098688 & -1.051093 \\
\hline 22 & 6 & 0 & -3.874006 & -1.860959 & -0.693232 \\
\hline 23 & 6 & 0 & -2.281669 & -1.155432 & -2.363241 \\
\hline 24 & 6 & 0 & -4.500104 & -2.666356 & -1.639951 \\
\hline 25 & 1 & 0 & -4.245701 & -1.837411 & 0.330399 \\
\hline 26 & 6 & 0 & -2.916018 & -1.949513 & -3.309280 \\
\hline 27 & 1 & 0 & -1.393237 & -0.581127 & -2.635136 \\
\hline 28 & 6 & 0 & -4.025477 & -2.709233 & -2.945938 \\
\hline 29 & 1 & 0 & -5.361150 & -3.267104 & -1.351827 \\
\hline 30 & 1 & 0 & -2.533810 & -1.990530 & -4.327634 \\
\hline 31 & 1 & 0 & -4.514786 & -3.343986 & -3.682768 \\
\hline 32 & 6 & 0 & -2.480317 & -0.481550 & 1.790753 \\
\hline 33 & 6 & 0 & -1.665864 & -1.275597 & 2.602093 \\
\hline 34 & 6 & 0 & -3.741967 & -0.089012 & 2.256494 \\
\hline 35 & 6 & 0 & -2.108821 & -1.675669 & 3.859201 \\
\hline 36 & 1 & 0 & -0.688558 & -1.594309 & 2.238160 \\
\hline 37 & 6 & 0 & -4.179866 & -0.491598 & 3.512161 \\
\hline 38 & 1 & 0 & -4.381553 & 0.537415 & 1.633999 \\
\hline 39 & 6 & 0 & -3.362899 & -1.284496 & 4.314562 \\
\hline 40 & 1 & 0 & -1.467933 & -2.296514 & 4.482532 \\
\hline 41 & 1 & 0 & -5.161406 & -0.181901 & 3.867278 \\
\hline 42 & 1 & 0 & -3.707409 & -1.597146 & 5.299081 \\
\hline 43 & 6 & 0 & -2.689249 & 1.632895 & -0.138432 \\
\hline 44 & 6 & 0 & -3.410566 & 1.933348 & -1.294092 \\
\hline 45 & 6 & 0 & -2.452774 & 2.642993 & 0.801620 \\
\hline 46 & 6 & 0 & -3.879938 & 3.227074 & -1.510995 \\
\hline 47 & 1 & 0 & -3.606482 & 1.156983 & -2.033098 \\
\hline 48 & 6 & 0 & -2.920325 & 3.930210 & 0.582756 \\
\hline 49 & 1 & 0 & -1.883979 & 2.421221 & 1.705149 \\
\hline 50 & 6 & 0 & -3.632630 & 4.226463 & -0.577754 \\
\hline 51 & 1 & 0 & -4.444096 & 3.450466 & -2.415224 \\
\hline 52 & 1 & 0 & -2.723349 & 4.707921 & 1.318727 \\
\hline 53 & 1 & 0 & -3.996378 & 5.237973 & -0.751304 \\
\hline 54 & 6 & 0 & 6.141161 & 0.096506 & 0.439717 \\
\hline 55 & 1 & 0 & 2.639658 & 0.845503 & -2.240580 \\
\hline 56 & 1 & 0 & 0.636131 & 5.884608 & -0.303536 \\
\hline 57 & 9 & 0 & 6.220066 & -0.875471 & 1.347516 \\
\hline 58 & 9 & 0 & 6.238977 & 1.265252 & 1.076056 \\
\hline 59 & 9 & 0 & 7.149788 & -0.019432 & -0.403290 \\
\hline \multicolumn{6}{|c|}{0.439166 (Hartree/Particle) } \\
\hline \multicolumn{3}{|c|}{ Thermal correction to Energy= } & \multicolumn{3}{|c|}{$\begin{array}{l}0.439166 \text { (Hartree/Particle) } \\
0.473435\end{array}$} \\
\hline \multicolumn{3}{|c|}{ Thermal correction to Enthalpy= } & & \\
\hline \multirow{2}{*}{\multicolumn{3}{|c|}{$\begin{array}{l}\text { Thermal correction to Gibbs Free Energy= } \\
\text { Sum of electronic and zero-point Energies= }\end{array}$}} & \multicolumn{2}{|c|}{$\begin{array}{c}0.474379 \\
0.367022\end{array}$} & \\
\hline & & & & 1971.644265 & \\
\hline Sum of el & tronic and $\mathrm{t}$ & mal Energies= & & -1971.609996 & \\
\hline Sum of el & tronic and $\mathrm{t}$ & mal Enthalpies= & & 1971.609052 & \\
\hline Sum of el & tronic and $\mathrm{t}$ & mal Free Energies & & 1971.716409 & \\
\hline
\end{tabular}

TS6ab 


\begin{tabular}{|c|c|c|c|c|c|}
\hline \multirow{2}{*}{$\begin{array}{l}\text { Center } \\
\text { Number }\end{array}$} & \multirow{2}{*}{$\begin{array}{l}\text { Atomic } \\
\text { Number }\end{array}$} & \multirow{2}{*}{$\begin{array}{l}\text { Atomic } \\
\text { Type }\end{array}$} & \multicolumn{3}{|c|}{ Coordinates (Angstroms) } \\
\hline & & & $\mathrm{X}$ & $\mathrm{Y}$ & $\mathrm{Z}$ \\
\hline 1 & 6 & 0 & -4.407281 & -0.421239 & -0.041872 \\
\hline 2 & 6 & 0 & -3.701495 & -0.457510 & -1.238781 \\
\hline 3 & 6 & 0 & -2.382004 & -0.024295 & -1.247047 \\
\hline 4 & 6 & 0 & -1.772118 & 0.439108 & -0.076156 \\
\hline 5 & 6 & 0 & -2.521181 & 0.496910 & 1.104387 \\
\hline 6 & 6 & 0 & -3.838702 & 0.057015 & 1.133388 \\
\hline 7 & 1 & 0 & -4.185058 & -0.842232 & -2.132744 \\
\hline 8 & 1 & 0 & -1.811345 & -0.087032 & -2.175963 \\
\hline 9 & 1 & 0 & -2.067070 & 0.875898 & 2.021409 \\
\hline 10 & 1 & 0 & -4.431046 & 0.073294 & 2.044586 \\
\hline 11 & 53 & 0 & 2.778937 & 1.935327 & -0.023629 \\
\hline 12 & 46 & 0 & 0.186403 & 1.012381 & -0.060547 \\
\hline 13 & 15 & 0 & 0.835817 & -1.282626 & 0.052029 \\
\hline 14 & 6 & 0 & -0.891222 & 3.916851 & 0.836706 \\
\hline 15 & 1 & 0 & -0.940607 & 3.838832 & 1.919672 \\
\hline 16 & 6 & 0 & -0.495709 & 2.970707 & -0.025071 \\
\hline 17 & 6 & 0 & -0.471321 & 3.177453 & -1.389334 \\
\hline 18 & 1 & 0 & 0.460555 & 3.440919 & -1.892200 \\
\hline 19 & 6 & 0 & -1.248804 & 5.148897 & 0.123310 \\
\hline 20 & 8 & 0 & -1.177635 & 5.190253 & -1.109565 \\
\hline 21 & 6 & 0 & 2.174463 & -1.689676 & -1.120172 \\
\hline 22 & 6 & 0 & 3.252143 & -2.505365 & -0.776825 \\
\hline 23 & 6 & 0 & 2.106275 & -1.142386 & -2.405776 \\
\hline 24 & 6 & 0 & 4.243801 & -2.779419 & -1.714253 \\
\hline 25 & 1 & 0 & 3.330056 & -2.915631 & 0.229191 \\
\hline 26 & 6 & 0 & 3.088597 & -1.430099 & -3.343524 \\
\hline 27 & 1 & 0 & 1.283699 & -0.470872 & -2.661597 \\
\hline 28 & 6 & 0 & 4.161032 & -2.248049 & -2.996187 \\
\hline 29 & 1 & 0 & 5.089182 & -3.406444 & -1.436355 \\
\hline 30 & 1 & 0 & 3.028149 & -0.999457 & -4.341304 \\
\hline 31 & 1 & 0 & 4.940651 & -2.460273 & -3.725778 \\
\hline 32 & 6 & 0 & 1.414081 & -1.792320 & 1.706368 \\
\hline 33 & 6 & 0 & 1.856948 & -0.823802 & 2.610446 \\
\hline 34 & 6 & 0 & 1.445813 & -3.143990 & 2.074227 \\
\hline 35 & 6 & 0 & 2.328937 & -1.202226 & 3.863614 \\
\hline 36 & 1 & 0 & 1.849271 & 0.227100 & 2.319089 \\
\hline 37 & 6 & 0 & 1.918775 & -3.516762 & 3.326120 \\
\hline 38 & 1 & 0 & 1.093942 & -3.905868 & 1.378031 \\
\hline 39 & 6 & 0 & 2.359989 & -2.545423 & 4.221837 \\
\hline 40 & 1 & 0 & 2.675765 & -0.441258 & 4.560214 \\
\hline 41 & 1 & 0 & 1.940200 & -4.568883 & 3.604826 \\
\hline 42 & 1 & 0 & 2.728009 & -2.839483 & 5.203465 \\
\hline 43 & 6 & 0 & -0.489454 & -2.486062 & -0.335227 \\
\hline 44 & 6 & 0 & -0.595594 & -3.074747 & -1.596788 \\
\hline 45 & 6 & 0 & -1.498545 & -2.711158 & 0.609135 \\
\hline 46 & 6 & 0 & -1.696118 & -3.867152 & -1.912000 \\
\hline 47 & 1 & 0 & 0.183792 & -2.916219 & -2.341462 \\
\hline 48 & 6 & 0 & -2.593141 & -3.504439 & 0.293039 \\
\hline 49 & 1 & 0 & -1.427720 & -2.255699 & 1.597263 \\
\hline 50 & 6 & 0 & -2.697835 & -4.079856 & -0.971822 \\
\hline 51 & 1 & 0 & -1.767587 & -4.321489 & -2.898891 \\
\hline 52 & 1 & 0 & -3.374447 & -3.665382 & 1.034695 \\
\hline 53 & 1 & 0 & -3.562559 & -4.691768 & -1.222281 \\
\hline 54 & 6 & 0 & -1.691278 & 6.339090 & 0.913917 \\
\hline 55 & 1 & 0 & -2.552947 & 6.073000 & 1.539898 \\
\hline 56 & 1 & 0 & -1.954224 & 7.170328 & 0.255365 \\
\hline 57 & 1 & 0 & -0.886829 & 6.647539 & 1.594594 \\
\hline 58 & 1 & 0 & -1.305903 & 2.852189 & -2.012962 \\
\hline 59 & 7 & 0 & -5.779749 & -0.924049 & -0.012470 \\
\hline 60 & 8 & 0 & -6.365857 & -0.913084 & 1.060404 \\
\hline 61 & 8 & 0 & -6.259944 & -1.332868 & -1.059630 \\
\hline
\end{tabular}

Zero-point correction= 0.463946 (Hartree/Particle)

Thermal correction to Energy= 0.499029

Thermal correction to Enthalpy=

Thermal correction to Gibbs Free Energy=

Sum of electronic and zero-point Energies

Sum of electronic and thermal Energies=

$$
0.499973
$$$$
0.391158
$$

$-1878.399149$

-1878.399149
-1878.364067

-1878.363122
-1878.471938

Sum of electronic and thermal Free Energies=

Z

M06 /6-311++G(d,p)-SDD/SMD//M06/6-31G(d)-LANL2DZ energy in toluene solvent = -1880.4994391

TS6ac

\begin{tabular}{|c|c|c|c|c|c|}
\hline \multirow{2}{*}{$\begin{array}{l}\text { Center } \\
\text { Number }\end{array}$} & \multirow{2}{*}{$\begin{array}{l}\text { Atomic } \\
\text { Number }\end{array}$} & \multirow{2}{*}{$\begin{array}{l}\text { Atomic } \\
\text { Type }\end{array}$} & \multicolumn{3}{|c|}{ Coordinates (Angstroms) } \\
\hline & & & $\mathrm{X}$ & $\mathrm{Y}$ & $\mathrm{Z}$ \\
\hline 1 & 6 & 0 & 3.726745 & 2.666660 & -0.244206 \\
\hline 2 & 6 & 0 & 2.973355 & 2.335883 & -1.373309 \\
\hline 3 & 6 & 0 & 2.041996 & 1.308757 & -1.312475 \\
\hline 4 & 6 & 0 & 1.840145 & 0.589691 & -0.129379 \\
\hline 5 & 6 & 0 & 2.619299 & 0.905840 & 0.978204 \\
\hline 6 & 6 & 0 & 3.555019 & 1.944629 & 0.935688 \\
\hline 7 & 1 & 0 & 3.124370 & 2.914349 & -2.283613 \\
\hline 8 & 1 & 0 & 1.440736 & 1.091787 & -2.198877 \\
\hline 9 & 1 & 0 & 2.501757 & 0.348512 & 1.909713 \\
\hline 10 & 1 & 0 & 4.139542 & 2.174620 & 1.824280 \\
\hline 11 & 53 & 0 & -1.306799 & -3.045819 & 0.100860 \\
\hline 12 & 46 & 0 & 0.435234 & -0.896618 & -0.007605 \\
\hline 13 & 15 & 0 & -1.302590 & 0.740728 & 0.060129 \\
\hline
\end{tabular}




\begin{tabular}{|c|c|c|c|c|c|}
\hline 14 & 6 & 0 & 2.883960 & -2.824289 & 0.823900 \\
\hline 15 & 1 & 0 & 2.923205 & -2.736315 & 1.906522 \\
\hline 16 & 6 & 0 & 2.026978 & -2.212231 & -0.007734 \\
\hline 17 & 6 & 0 & 2.081758 & -2.413033 & -1.374689 \\
\hline 18 & 1 & 0 & 1.403511 & -3.121212 & -1.853544 \\
\hline 19 & 6 & 0 & 3.796155 & -3.690494 & 0.076814 \\
\hline 20 & 8 & 0 & 3.713058 & -3.758413 & -1.156332 \\
\hline 21 & 6 & 0 & -2.583532 & 0.422344 & -1.202010 \\
\hline 22 & 6 & 0 & -3.933811 & 0.710132 & -1.001667 \\
\hline 23 & 6 & 0 & -2.163344 & -0.083265 & -2.435756 \\
\hline 24 & 6 & 0 & -4.850555 & 0.498828 & -2.027379 \\
\hline 25 & 1 & 0 & -4.276177 & 1.089186 & -0.039129 \\
\hline 26 & 6 & 0 & -3.077725 & -0.280540 & -3.462471 \\
\hline 27 & 1 & 0 & -1.111188 & -0.338497 & -2.579501 \\
\hline 28 & 6 & 0 & -4.424238 & 0.009073 & -3.257086 \\
\hline 29 & 1 & 0 & -5.904638 & 0.714939 & -1.861078 \\
\hline 30 & 1 & 0 & -2.741230 & -0.675619 & -4.419333 \\
\hline 31 & 1 & 0 & -5.145132 & -0.156220 & -4.056117 \\
\hline 32 & 6 & 0 & -2.176839 & 0.898110 & 1.659242 \\
\hline 33 & 6 & 0 & -2.160850 & -0.160636 & 2.570710 \\
\hline 34 & 6 & 0 & -2.876130 & 2.069221 & 1.979176 \\
\hline 35 & 6 & 0 & -2.841786 & -0.051622 & 3.779637 \\
\hline 36 & 1 & 0 & -1.622745 & -1.076250 & 2.323630 \\
\hline 37 & 6 & 0 & -3.556189 & 2.172530 & 3.186301 \\
\hline 38 & 1 & 0 & -2.880208 & 2.907403 & 1.281764 \\
\hline 39 & 6 & 0 & -3.539403 & 1.110958 & 4.087838 \\
\hline 40 & 1 & 0 & -2.824789 & -0.883326 & 4.481595 \\
\hline 41 & 1 & 0 & -4.097876 & 3.086092 & 3.426121 \\
\hline 42 & 1 & 0 & -4.069219 & 1.193986 & 5.035634 \\
\hline 43 & 6 & 0 & -0.784182 & 2.462937 & -0.287969 \\
\hline 44 & 6 & 0 & -1.113641 & 3.117854 & -1.475044 \\
\hline 45 & 6 & 0 & 0.056987 & 3.099464 & 0.632564 \\
\hline 46 & 6 & 0 & -0.599239 & 4.385329 & -1.742255 \\
\hline 47 & 1 & 0 & -1.771990 & 2.639471 & -2.199523 \\
\hline 48 & 6 & 0 & 0.570615 & 4.359108 & 0.362522 \\
\hline 49 & 1 & 0 & 0.329594 & 2.592000 & 1.558429 \\
\hline 50 & 6 & 0 & 0.246649 & 5.003916 & -0.830104 \\
\hline 51 & 1 & 0 & -0.862963 & 4.887999 & -2.671549 \\
\hline 52 & 1 & 0 & 1.240807 & 4.833213 & 1.078632 \\
\hline 53 & 1 & 0 & 0.657422 & 5.988868 & -1.045884 \\
\hline 54 & 6 & 0 & 4.815828 & -4.491459 & 0.823141 \\
\hline 55 & 1 & 0 & 4.309089 & -5.173889 & 1.518207 \\
\hline 56 & 1 & 0 & 5.443647 & -3.827269 & 1.431164 \\
\hline 57 & 1 & 0 & 5.441042 & -5.066636 & 0.135866 \\
\hline 58 & 1 & 0 & 2.606805 & -1.703045 & -2.015766 \\
\hline 59 & 8 & 0 & 4.597730 & 3.702075 & -0.394749 \\
\hline 60 & 6 & 0 & 5.343532 & 4.086233 & 0.728188 \\
\hline 61 & 1 & 0 & 5.961409 & 4.933819 & 0.418666 \\
\hline 62 & 1 & 0 & 4.692997 & 4.400293 & 1.560542 \\
\hline 63 & 1 & 0 & 5.999006 & 3.274796 & 1.081772 \\
\hline
\end{tabular}

Zero-point correction= 0.493895 (Hartree/Particle) Thermal correction to Energy= Thermal correction to Enthalpy

Thermal correction to Gibbs Free Energy=

Sum of electronic and zero-point Energies

Sum of electronic and thermal Energies $=$

Sum of electronic and thermal Enthalpies=

Sum of electronic and thermal Free Energies=

$$
\begin{aligned}
& 0.493895 \\
& 0.528867
\end{aligned}
$$

$$
\begin{gathered}
0.528867 \\
0.529811 \\
0.422779
\end{gathered}
$$

$-1788.412134$

$-1788.377162$

$-1788.376218$

$-1788.483250$

M06 /6-311++G(d,p)-SDD/SMD//M06/6-31G(d)-LANL2DZ energy in toluene solvent $=-1790.5150058$

\section{INT2ab}

\begin{tabular}{rrrrrr} 
Center & Atomic & Atomic & \multicolumn{2}{c}{ Coordinates (Angstroms) } \\
Number & Number & Type & X & Y & $Z$ \\
-1 & 46 & 0 & 0.064588 & -0.276442 & -0.001363 \\
2 & 15 & 0 & -2.308251 & -0.462703 & -0.019995 \\
3 & 15 & 0 & 2.419611 & 0.005968 & -0.002419 \\
4 & 6 & 0 & -0.145224 & 1.754752 & -0.080276 \\
5 & 6 & 0 & -0.519077 & 2.474700 & 1.050906 \\
6 & 1 & 0 & -0.684326 & 1.961009 & 2.001327 \\
7 & 6 & 0 & 0.039743 & 2.455111 & -1.277434 \\
8 & 6 & 0 & -0.732446 & 3.854414 & 1.002058 \\
9 & 6 & 0 & -0.578910 & 4.528416 & -0.208308 \\
10 & 6 & 0 & -0.174228 & 3.825393 & -1.345746 \\
11 & 1 & 0 & -0.044721 & 4.376499 & -2.276370 \\
12 & 1 & 0 & 0.359782 & 1.927612 & -2.179273 \\
13 & 1 & 0 & -1.048458 & 4.378467 & 1.901901 \\
14 & 6 & 0 & -3.273584 & 1.072724 & 0.262756 \\
15 & 6 & 0 & -3.903144 & 1.346735 & 1.477821 \\
16 & 6 & 0 & -3.284162 & 2.047042 & -0.742680 \\
17 & 6 & 0 & -4.524116 & 2.576255 & 1.687013 \\
18 & 1 & 0 & -3.913830 & 0.598149 & 2.269039 \\
19 & 6 & 0 & -3.900081 & 3.272584 & -0.531300 \\
20 & 1 & 0 & -2.787961 & 1.852725 & -1.693824 \\
21 & 6 & 0 & -4.520884 & 3.541313 & 0.687505 \\
22 & 1 & 0 & -5.015956 & 2.774176 & 2.638380 \\
23 & 1 & 0 & -3.880883 & 4.027226 & -1.316673 \\
24 & 1 & 0 & -5.004150 & 4.503465 & 0.853444 \\
25 & 6 & 0 & -2.903848 & -1.596955 & 1.282216 \\
26 & 6 & 0 & -3.858742 & -2.586089 & 1.052392 \\
27 & 6 & 0 & -2.346478 & -1.463440 & 2.558682
\end{tabular}




\begin{tabular}{|c|c|c|c|c|c|}
\hline 28 & 6 & 0 & -4.256478 & -3.425797 & 2.089215 \\
\hline 29 & 1 & 0 & -4.280303 & -2.717667 & 0.056612 \\
\hline 30 & 6 & 0 & -2.757068 & -2.290681 & 3.595054 \\
\hline 31 & 1 & 0 & -1.570357 & -0.713827 & 2.731896 \\
\hline 32 & 6 & 0 & -3.711959 & -3.277033 & 3.359213 \\
\hline 33 & 1 & 0 & -4.993628 & -4.204235 & 1.899294 \\
\hline 34 & 1 & 0 & -2.317808 & -2.178641 & 4.584934 \\
\hline 35 & 1 & 0 & -4.023486 & -3.937106 & 4.167100 \\
\hline 36 & 6 & 0 & -2.991916 & -1.072339 & -1.602187 \\
\hline 37 & 6 & 0 & -4.357046 & -0.942994 & -1.888231 \\
\hline 38 & 6 & 0 & -2.147385 & -1.661954 & -2.545868 \\
\hline 39 & 6 & 0 & -4.868366 & -1.407375 & -3.093745 \\
\hline 40 & 1 & 0 & -5.022033 & -0.470588 & -1.164404 \\
\hline 41 & 6 & 0 & -2.663045 & -2.122094 & -3.754383 \\
\hline 42 & 1 & 0 & -1.086250 & -1.777019 & -2.319997 \\
\hline 43 & 6 & 0 & -4.020247 & -1.996583 & -4.028456 \\
\hline 44 & 1 & 0 & -5.931302 & -1.304801 & -3.306299 \\
\hline 45 & 1 & 0 & -1.997169 & -2.582580 & -4.482040 \\
\hline 46 & 1 & 0 & -4.420736 & -2.357317 & -4.974741 \\
\hline 47 & 6 & 0 & 3.201891 & -1.106665 & -1.220158 \\
\hline 48 & 6 & 0 & 4.295924 & -1.915628 & -0.918850 \\
\hline 49 & 6 & 0 & 2.625863 & -1.175018 & -2.493690 \\
\hline 50 & 6 & 0 & 4.806880 & -2.783569 & -1.880019 \\
\hline 51 & 1 & 0 & 4.738389 & -1.886589 & 0.076109 \\
\hline 52 & 6 & 0 & 3.146103 & -2.030228 & -3.454750 \\
\hline 53 & 1 & 0 & 1.747625 & -0.566032 & -2.722045 \\
\hline 54 & 6 & 0 & 4.236150 & -2.840983 & -3.145951 \\
\hline 55 & 1 & 0 & 5.652298 & -3.423184 & -1.632048 \\
\hline 56 & 1 & 0 & 2.689052 & -2.080417 & -4.441586 \\
\hline 57 & 1 & 0 & 4.633997 & -3.526421 & -3.892509 \\
\hline 58 & 6 & 0 & 3.210844 & -0.336586 & 1.608820 \\
\hline 59 & 6 & 0 & 4.526196 & 0.072448 & 1.862529 \\
\hline 60 & 6 & 0 & 2.495752 & -1.005063 & 2.605003 \\
\hline 61 & 6 & 0 & 5.117715 & -0.193522 & 3.091173 \\
\hline 62 & 1 & 0 & 5.084985 & 0.606604 & 1.092895 \\
\hline 63 & 6 & 0 & 3.090363 & -1.265209 & 3.836174 \\
\hline 64 & 1 & 0 & 1.476969 & -1.338445 & 2.401449 \\
\hline 65 & 6 & 0 & 4.398450 & -0.861456 & 4.079726 \\
\hline 66 & 1 & 0 & 6.141403 & 0.125596 & 3.280146 \\
\hline 67 & 1 & 0 & 2.527155 & -1.789513 & 4.606165 \\
\hline 68 & 1 & 0 & 4.861112 & -1.064502 & 5.044460 \\
\hline 69 & 6 & 0 & 3.068698 & 1.666315 & -0.425309 \\
\hline 70 & 6 & 0 & 3.693250 & 1.938591 & -1.642746 \\
\hline 71 & 6 & 0 & 2.833340 & 2.716520 & 0.470511 \\
\hline 72 & 6 & 0 & 4.064542 & 3.242341 & -1.965809 \\
\hline 73 & 1 & 0 & 3.890842 & 1.132389 & -2.347987 \\
\hline 74 & 6 & 0 & 3.202300 & 4.013346 & 0.145794 \\
\hline 75 & 1 & 0 & 2.329649 & 2.520444 & 1.417464 \\
\hline 76 & 6 & 0 & 3.815783 & 4.280369 & -1.076957 \\
\hline 77 & 1 & 0 & 4.550885 & 3.441910 & -2.919516 \\
\hline 78 & 1 & 0 & 2.997996 & 4.822453 & 0.845291 \\
\hline 79 & 1 & 0 & 4.096463 & 5.300330 & -1.334810 \\
\hline 80 & 53 & 0 & 0.333216 & -3.046898 & 0.121681 \\
\hline 81 & 8 & 0 & -0.803157 & 5.861593 & -0.382126 \\
\hline 82 & 6 & 0 & -1.311869 & 6.575359 & 0.711324 \\
\hline 83 & 1 & 0 & -1.469943 & 7.601890 & 0.368350 \\
\hline 84 & 1 & 0 & -2.270935 & 6.156517 & 1.057729 \\
\hline 85 & 1 & 0 & -0.607906 & 6.586817 & 1.558747 \\
\hline
\end{tabular}

Zero-point correction=

0.673181 (Hartree/Particle)

Thermal correction to Energy=

Thermal correction to Enthalpy=

Thermal correction to Gibbs Free Energy=

Sum of electronic and zero-point Energies

Sum of electronic and thermal Energies $=$

Sum of electronic and thermal Enthalpies=

Sum of electronic and thermal Free Energies=

0.718335

\subsection{719279}

0.590066

$-2554.863145$

$-2554.81799$

$-2554.817047$

$-2554.946260$

M06 /6-311++G(d,p)-SDD/SMD//M06/6-31G(d)-LANL2DZ energy in toluene solvent $=-2557.2879126$

\section{INT2ac}

\begin{tabular}{rrrrrr} 
Center & Atomic & Atomic & \multicolumn{2}{c}{ Coordinates (Angstroms) } \\
Number & Number & Type & $\mathrm{X}$ & $\mathrm{Y}$ & $\mathrm{Z}$ \\
$-\mathrm{-y}$ & 46 & 0 & -0.065131 & -0.356643 & -0.024120 \\
2 & 15 & 0 & -2.424231 & -0.062422 & 0.000074 \\
3 & 15 & 0 & 2.304670 & -0.599486 & -0.003377 \\
4 & 6 & 0 & 0.176278 & 1.668900 & -0.036576 \\
5 & 6 & 0 & -0.001040 & 2.418916 & 1.133122 \\
6 & 1 & 0 & -0.311507 & 1.928614 & 2.057404 \\
7 & 6 & 0 & 0.552401 & 2.336656 & -1.209695 \\
8 & 6 & 0 & 0.206171 & 3.791439 & 1.145612 \\
9 & 6 & 0 & 0.602686 & 4.416713 & -0.031989 \\
10 & 6 & 0 & 0.770355 & 3.707581 & -1.216930 \\
11 & 1 & 0 & 1.082185 & 4.233884 & -2.115330 \\
12 & 1 & 0 & 0.708638 & 1.777238 & -2.134056 \\
13 & 1 & 0 & 0.073191 & 4.383161 & 2.047435 \\
14 & 6 & 0 & -3.017455 & 1.639085 & 0.327097 \\
15 & 6 & 0 & -3.510439 & 2.039507 & 1.569490 \\
16 & 6 & 0 & -2.837439 & 2.598580 & -0.676781 \\
17 & 6 & 0 & -3.802843 & 3.380493 & 1.809020 \\
18 & 1 & 0 & -3.666076 & 1.304296 & 2.358243 \\
19 & 6 & 0 & -3.128436 & 3.933307 & -0.435326
\end{tabular}




\begin{tabular}{|c|c|c|c|c|c|}
\hline 20 & 1 & 0 & -2.449975 & 2.297686 & -1.650812 \\
\hline 21 & 6 & 0 & -3.605779 & 4.329070 & 0.812734 \\
\hline 22 & 1 & 0 & -4.186294 & 3.682270 & 2.782405 \\
\hline 23 & 1 & 0 & -2.973153 & 4.671094 & -1.220934 \\
\hline 24 & 1 & 0 & -3.823567 & 5.378082 & 1.005672 \\
\hline 25 & 6 & 0 & -3.189604 & -1.077938 & 1.308808 \\
\hline 26 & 6 & 0 & -4.296617 & -1.895098 & 1.086667 \\
\hline 27 & 6 & 0 & -2.590144 & -1.060760 & 2.573363 \\
\hline 28 & 6 & 0 & -4.803159 & -2.678070 & 2.120404 \\
\hline 29 & 1 & 0 & -4.754055 & -1.941212 & 0.099281 \\
\hline 30 & 6 & 0 & -3.106182 & -1.831038 & 3.606160 \\
\hline 31 & 1 & 0 & -1.697983 & -0.452927 & 2.740524 \\
\hline 32 & 6 & 0 & -4.213928 & -2.644306 & 3.378793 \\
\hline 33 & 1 & 0 & -5.659709 & -3.323844 & 1.935202 \\
\hline 34 & 1 & 0 & -2.633299 & -1.810799 & 4.586512 \\
\hline 35 & 1 & 0 & -4.610190 & -3.261650 & 4.183161 \\
\hline 36 & 6 & 0 & -3.255640 & -0.486676 & -1.568427 \\
\hline 37 & 6 & 0 & -4.581965 & -0.098887 & -1.798568 \\
\hline 38 & 6 & 0 & -2.564694 & -1.193561 & -2.554830 \\
\hline 39 & 6 & 0 & -5.209765 & -0.429843 & -2.992681 \\
\hline 40 & 1 & 0 & -5.119126 & 0.470245 & -1.038684 \\
\hline 41 & 6 & 0 & -3.195610 & -1.517319 & -3.752344 \\
\hline 42 & 1 & 0 & -1.536275 & -1.507277 & -2.369807 \\
\hline 43 & 6 & 0 & -4.515645 & -1.139049 & -3.970621 \\
\hline 44 & 1 & 0 & -6.241858 & -0.128707 & -3.163893 \\
\hline 45 & 1 & 0 & -2.651545 & -2.070935 & -4.515365 \\
\hline 46 & 1 & 0 & -5.007720 & -1.394046 & -4.907917 \\
\hline 47 & 6 & 0 & 2.860872 & -1.789917 & -1.269490 \\
\hline 48 & 6 & 0 & 3.765756 & -2.815477 & -1.000169 \\
\hline 49 & 6 & 0 & 2.310322 & -1.676837 & -2.550976 \\
\hline 50 & 6 & 0 & 4.122004 & -3.711295 & -2.004446 \\
\hline 51 & 1 & 0 & 4.178915 & -2.931882 & 0.000891 \\
\hline 52 & 6 & 0 & 2.680450 & -2.561024 & -3.554986 \\
\hline 53 & 1 & 0 & 1.568284 & -0.900784 & -2.753739 \\
\hline 54 & 6 & 0 & 3.585744 & -3.583378 & -3.280202 \\
\hline 55 & 1 & 0 & 4.818954 & -4.517900 & -1.783627 \\
\hline 56 & 1 & 0 & 2.247262 & -2.464746 & -4.549078 \\
\hline 57 & 1 & 0 & 3.864352 & -4.288043 & -4.061878 \\
\hline 58 & 6 & 0 & 2.974878 & -1.163155 & 1.599424 \\
\hline 59 & 6 & 0 & 4.346341 & -1.068474 & 1.868610 \\
\hline 60 & 6 & 0 & 2.118445 & -1.679828 & 2.574090 \\
\hline 61 & 6 & 0 & 4.851303 & -1.496457 & 3.090099 \\
\hline 62 & 1 & 0 & 5.020259 & -0.651597 & 1.119353 \\
\hline 63 & 6 & 0 & 2.628086 & -2.103695 & 3.798280 \\
\hline 64 & 1 & 0 & 1.052276 & -1.766656 & 2.361482 \\
\hline 65 & 6 & 0 & 3.991200 & -2.013797 & 4.056056 \\
\hline 66 & 1 & 0 & 5.918771 & -1.422003 & 3.290541 \\
\hline 67 & 1 & 0 & 1.953231 & -2.508715 & 4.550161 \\
\hline 68 & 1 & 0 & 4.387588 & -2.346838 & 5.014006 \\
\hline 69 & 6 & 0 & 3.284747 & 0.912860 & -0.337363 \\
\hline 70 & 6 & 0 & 3.888792 & 1.159322 & -1.571134 \\
\hline 71 & 6 & 0 & 3.322870 & 1.905027 & 0.649931 \\
\hline 72 & 6 & 0 & 4.507538 & 2.383105 & -1.817489 \\
\hline 73 & 1 & 0 & 3.879258 & 0.395101 & -2.347356 \\
\hline 74 & 6 & 0 & 3.937696 & 3.123861 & 0.401252 \\
\hline 75 & 1 & 0 & 2.857271 & 1.725093 & 1.619555 \\
\hline 76 & 6 & 0 & 4.527211 & 3.368396 & -0.837883 \\
\hline 77 & 1 & 0 & 4.976478 & 2.562747 & -2.783696 \\
\hline 78 & 1 & 0 & 3.946147 & 3.891866 & 1.173451 \\
\hline 79 & 1 & 0 & 4.999932 & 4.328775 & -1.036084 \\
\hline 80 & 53 & 0 & -0.382040 & -3.113650 & -0.028601 \\
\hline 81 & 7 & 0 & 0.856092 & 5.853863 & -0.022953 \\
\hline 82 & 8 & 0 & 1.206070 & 6.379419 & -1.070724 \\
\hline 83 & 8 & 0 & 0.708896 & 6.453005 & 1.033470 \\
\hline
\end{tabular}

Zero-point correction= 4 (Hartree/Particle)

Thermal correction to Energy=

0.688966

Thermal correction to Enthalpy=

Thermal correction to Gibbs Free Energy=

Sum of electronic and zero-point Energies

Sum of electronic and thermal Energies=

$$
0.689910
$$$$
0.560143
$$

$-2644.850544$

$-2644.805482$

$-2644.804538$

Sum of electronic and thermal Free Energies=

Z. 934305

M06 /6-311++G(d,p)-SDD/SMD//M06/6-31G(d)-LANL2DZ energy in toluene solvent $=-2647.2729117$

\section{INT4ba}

\begin{tabular}{|c|c|c|c|c|c|}
\hline \multirow{2}{*}{$\begin{array}{l}\text { Center } \\
\text { Number }\end{array}$} & \multirow{2}{*}{$\begin{array}{l}\text { Atomic } \\
\text { Number }\end{array}$} & \multirow{2}{*}{$\begin{array}{l}\text { Atomic } \\
\text { Type }\end{array}$} & \multicolumn{3}{|c|}{ Coordinates (Angstroms) } \\
\hline & & & $\mathrm{X}$ & $\mathrm{Y}$ & $\mathrm{Z}$ \\
\hline 1 & 6 & 0 & 2.162988 & 3.738445 & 1.628078 \\
\hline 2 & 6 & 0 & 1.808144 & 3.617057 & 0.286700 \\
\hline 3 & 6 & 0 & 1.267176 & 2.424521 & -0.188325 \\
\hline 4 & 6 & 0 & 1.092551 & 1.339932 & 0.669748 \\
\hline 5 & 6 & 0 & 1.429380 & 1.468494 & 2.015983 \\
\hline 6 & 6 & 0 & 1.966680 & 2.665785 & 2.492580 \\
\hline 7 & 1 & 0 & 1.950807 & 4.453774 & -0.397070 \\
\hline 8 & 1 & 0 & 0.988144 & 2.343444 & -1.241443 \\
\hline 9 & 1 & 0 & 1.279584 & 0.634661 & 2.706371 \\
\hline 10 & 1 & 0 & 2.234994 & 2.755712 & 3.545423 \\
\hline 11 & 53 & 0 & -0.420225 & -2.936079 & -0.958531 \\
\hline 12 & 46 & 0 & 0.451283 & -0.461617 & -0.055158 \\
\hline \multirow[t]{2}{*}{13} & 15 & 0 & -1.740650 & 0.374968 & 0.097757 \\
\hline & & & & S80 & \\
\hline
\end{tabular}




\begin{tabular}{|c|c|c|c|c|c|}
\hline 14 & 6 & 0 & 2.723394 & -1.867242 & 1.556205 \\
\hline 15 & 1 & 0 & 2.003169 & -1.875796 & 2.376603 \\
\hline 16 & 6 & 0 & 2.434611 & -1.331912 & 0.385499 \\
\hline 17 & 6 & 0 & 2.672693 & -0.962717 & -0.891469 \\
\hline 18 & 1 & 0 & 2.334964 & -1.652378 & -1.671794 \\
\hline 19 & 6 & 0 & 4.053241 & -2.514613 & 1.759671 \\
\hline 20 & 8 & 0 & 4.941630 & -2.446494 & 0.935445 \\
\hline 21 & 6 & 0 & -2.693209 & -0.030442 & -1.403328 \\
\hline 22 & 6 & 0 & -3.963970 & -0.601853 & -1.362060 \\
\hline 23 & 6 & 0 & -2.087849 & 0.219786 & -2.639996 \\
\hline 24 & 6 & 0 & -4.626369 & -0.908557 & -2.546919 \\
\hline 25 & 1 & 0 & -4.430835 & -0.831587 & -0.405312 \\
\hline 26 & 6 & 0 & -2.757152 & -0.074416 & -3.819514 \\
\hline 27 & 1 & 0 & -1.073787 & 0.625888 & -2.673388 \\
\hline 28 & 6 & 0 & -4.028131 & -0.642763 & -3.773036 \\
\hline 29 & 1 & 0 & -5.612969 & -1.366949 & -2.508020 \\
\hline 30 & 1 & 0 & -2.278681 & 0.118980 & -4.777893 \\
\hline 31 & 1 & 0 & -4.547499 & -0.889958 & -4.697333 \\
\hline 32 & 6 & 0 & -2.628841 & -0.317589 & 1.531747 \\
\hline 33 & 6 & 0 & -2.073653 & -1.381626 & 2.245358 \\
\hline 34 & 6 & 0 & -3.856874 & 0.219383 & 1.939321 \\
\hline 35 & 6 & 0 & -2.742303 & -1.910770 & 3.345320 \\
\hline 36 & 1 & 0 & -1.121493 & -1.804196 & 1.919844 \\
\hline 37 & 6 & 0 & -4.524120 & -0.315664 & 3.033949 \\
\hline 38 & 1 & 0 & -4.286874 & 1.065201 & 1.401655 \\
\hline 39 & 6 & 0 & -3.966336 & -1.380872 & 3.737637 \\
\hline 40 & 1 & 0 & -2.303703 & -2.742860 & 3.892826 \\
\hline 41 & 1 & 0 & -5.479825 & 0.103632 & 3.343711 \\
\hline 42 & 1 & 0 & -4.488801 & -1.796675 & 4.597685 \\
\hline 43 & 6 & 0 & -1.971562 & 2.179598 & 0.308795 \\
\hline 44 & 6 & 0 & -2.335088 & 3.009466 & -0.752591 \\
\hline 45 & 6 & 0 & -1.694723 & 2.749147 & 1.557420 \\
\hline 46 & 6 & 0 & -2.414116 & 4.388073 & -0.570194 \\
\hline 47 & 1 & 0 & -2.564773 & 2.582305 & -1.727759 \\
\hline 48 & 6 & 0 & -1.779420 & 4.122347 & 1.736935 \\
\hline 49 & 1 & 0 & -1.398168 & 2.114617 & 2.392638 \\
\hline 50 & 6 & 0 & -2.134715 & 4.946295 & 0.670927 \\
\hline 51 & 1 & 0 & -2.700553 & 5.024611 & -1.405806 \\
\hline 52 & 1 & 0 & -1.556321 & 4.553139 & 2.711595 \\
\hline 53 & 1 & 0 & -2.194741 & 6.024307 & 0.811213 \\
\hline 54 & 6 & 0 & 4.209916 & -3.246714 & 3.066884 \\
\hline 55 & 1 & 0 & 4.039564 & -2.563206 & 3.910169 \\
\hline 56 & 1 & 0 & 5.212340 & -3.677295 & 3.137634 \\
\hline 57 & 1 & 0 & 3.457700 & -4.043402 & 3.147166 \\
\hline 58 & 1 & 0 & 2.585003 & 4.670797 & 2.001115 \\
\hline 59 & 6 & 0 & 3.382955 & 0.242405 & -1.313585 \\
\hline 60 & 6 & 0 & 3.212697 & 0.707768 & -2.622223 \\
\hline 61 & 6 & 0 & 4.224184 & 0.937559 & -0.437395 \\
\hline 62 & 6 & 0 & 3.859543 & 1.861795 & -3.044624 \\
\hline 63 & 1 & 0 & 2.556505 & 0.158251 & -3.299039 \\
\hline 64 & 6 & 0 & 4.871381 & 2.086634 & -0.863303 \\
\hline 65 & 1 & 0 & 4.367558 & 0.558155 & 0.573270 \\
\hline 66 & 6 & 0 & 4.686597 & 2.554222 & -2.163680 \\
\hline 67 & 1 & 0 & 3.721251 & 2.221585 & -4.062709 \\
\hline 68 & 1 & 0 & 5.522579 & 2.625350 & -0.177411 \\
\hline 69 & 1 & 0 & 5.196259 & 3.458148 & -2.493534 \\
\hline
\end{tabular}

Zero-point correction= 0.541577 (Hartree/Particle)

0.579722

Thermal correction to Enthalpy=

0.580666

Thermal correction to Gibbs Free Energy=

Sum of electronic and zero-point Energies=

Sum of electronic and thermal Energies=

0.465682

$-1904.794366$

Sum of electronic and thermal Enthalpies=

Sum of electronic and thermal Free Energies=

$-1904.756220$

$-1904.755276$

$-1904.870260$

M06 /6-311++G(d,p)-SDD/SMD//M06/6-31G(d)-LANL2DZ energy in toluene solvent $=-1906.9800691$

\section{INT4ca}

\begin{tabular}{rrrrrr} 
Center & Atomic & Atomic & \multicolumn{2}{c}{ Coordinates (Angstroms) } \\
Number & Number & Type & X & Y & $Z$ \\
--1 & 6 & 0 & -2.509242 & 2.457035 & -2.718226 \\
2 & 6 & 0 & -2.372524 & 2.677517 & -1.349829 \\
3 & 6 & 0 & -1.622331 & 1.799993 & -0.570480 \\
4 & 6 & 0 & -1.001827 & 0.694684 & -1.152559 \\
5 & 6 & 0 & -1.121317 & 0.488193 & -2.526518 \\
6 & 6 & 0 & -1.878218 & 1.364327 & -3.306076 \\
7 & 1 & 0 & -2.861153 & 3.532106 & -0.881329 \\
8 & 1 & 0 & -1.524122 & 1.985984 & 0.501838 \\
9 & 1 & 0 & -0.629704 & -0.364659 & -3.001043 \\
10 & 1 & 0 & -1.974864 & 1.188782 & -4.377740 \\
11 & 53 & 0 & 1.414951 & -2.593920 & 1.400617 \\
12 & 46 & 0 & 0.008828 & -0.672459 & -0.018176 \\
13 & 15 & 0 & 1.847203 & 0.803142 & -0.028246 \\
14 & 6 & 0 & -1.493875 & -2.966766 & -1.512945 \\
15 & 1 & 0 & -0.696318 & -2.915912 & -2.256690 \\
16 & 6 & 0 & -1.528645 & -2.155416 & -0.470136 \\
17 & 6 & 0 & -2.109160 & -1.707210 & 0.671153 \\
18 & 1 & 0 & -1.716741 & -2.116582 & 1.608182 \\
19 & 6 & 0 & -2.545172 & -4.014540 & -1.659401 \\
20 & 8 & 0 & -3.501463 & -4.087304 & -0.914384 \\
21 & 6 & 0 & 2.632579 & 0.872650 & 1.614988
\end{tabular}




\begin{tabular}{|c|c|c|c|c|c|}
\hline 22 & 6 & 0 & 3.993823 & 0.650253 & 1.816932 \\
\hline 23 & 6 & 0 & 1.797510 & 1.087661 & 2.717116 \\
\hline 24 & 6 & 0 & 4.512977 & 0.645123 & 3.108533 \\
\hline 25 & 1 & 0 & 4.647330 & 0.448156 & 0.969382 \\
\hline 26 & 6 & 0 & 2.321516 & 1.099495 & 4.001786 \\
\hline 27 & 1 & 0 & 0.722816 & 1.214806 & 2.565587 \\
\hline 28 & 6 & 0 & 3.681669 & 0.872655 & 4.198854 \\
\hline 29 & 1 & 0 & 5.573443 & 0.452233 & 3.261163 \\
\hline 30 & 1 & 0 & 1.664191 & 1.262449 & 4.853881 \\
\hline 31 & 1 & 0 & 4.090748 & 0.860341 & 5.207625 \\
\hline 32 & 6 & 0 & 3.114536 & 0.287001 & -1.231944 \\
\hline 33 & 6 & 0 & 3.014456 & -0.958832 & -1.855218 \\
\hline 34 & 6 & 0 & 4.178068 & 1.138275 & -1.557156 \\
\hline 35 & 6 & 0 & 3.972524 & -1.353130 & -2.784771 \\
\hline 36 & 1 & 0 & 2.188978 & -1.623905 & -1.593973 \\
\hline 37 & 6 & 0 & 5.135871 & 0.738168 & -2.480235 \\
\hline 38 & 1 & 0 & 4.249586 & 2.121448 & -1.090047 \\
\hline 39 & 6 & 0 & 5.032311 & -0.508181 & -3.094514 \\
\hline 40 & 1 & 0 & 3.889402 & -2.326820 & -3.264033 \\
\hline 41 & 1 & 0 & 5.962839 & 1.402132 & -2.725946 \\
\hline 42 & 1 & 0 & 5.781389 & -0.819410 & -3.820897 \\
\hline 43 & 6 & 0 & 1.537349 & 2.549759 & -0.481390 \\
\hline 44 & 6 & 0 & 1.438096 & 3.563610 & 0.472411 \\
\hline 45 & 6 & 0 & 1.276881 & 2.846672 & -1.824502 \\
\hline 46 & 6 & 0 & 1.070208 & 4.851303 & 0.089755 \\
\hline 47 & 1 & 0 & 1.651538 & 3.353690 & 1.519693 \\
\hline 48 & 6 & 0 & 0.911205 & 4.129917 & -2.202410 \\
\hline 49 & 1 & 0 & 1.340549 & 2.059933 & -2.576352 \\
\hline 50 & 6 & 0 & 0.800955 & 5.134977 & -1.243653 \\
\hline 51 & 1 & 0 & 0.996008 & 5.634949 & 0.842164 \\
\hline 52 & 1 & 0 & 0.701013 & 4.344377 & -3.248917 \\
\hline 53 & 1 & 0 & 0.507471 & 6.141073 & -1.539005 \\
\hline 54 & 6 & 0 & -2.334359 & -4.973040 & -2.803129 \\
\hline 55 & 1 & 0 & -3.158501 & -5.689953 & -2.847679 \\
\hline 56 & 1 & 0 & -1.383812 & -5.508774 & -2.674552 \\
\hline 57 & 1 & 0 & -2.265090 & -4.427347 & -3.754263 \\
\hline 58 & 1 & 0 & -3.102199 & 3.138659 & -3.326727 \\
\hline 59 & 6 & 0 & -3.199094 & -0.753489 & 0.764987 \\
\hline 60 & 6 & 0 & -3.468008 & -0.143336 & 1.992613 \\
\hline 61 & 6 & 0 & -3.992629 & -0.416369 & -0.344857 \\
\hline 62 & 6 & 0 & -4.487174 & 0.790657 & 2.127311 \\
\hline 63 & 1 & 0 & -2.855353 & -0.401586 & 2.857358 \\
\hline 64 & 6 & 0 & -5.012012 & 0.501070 & -0.222656 \\
\hline 65 & 1 & 0 & -3.791569 & -0.885028 & -1.307331 \\
\hline 66 & 6 & 0 & -5.263215 & 1.117230 & 1.012167 \\
\hline 67 & 1 & 0 & -4.670285 & 1.249427 & 3.095470 \\
\hline 68 & 1 & 0 & -5.632213 & 0.779289 & -1.072033 \\
\hline 69 & 8 & 0 & -6.277787 & 2.008311 & 1.023687 \\
\hline 70 & 6 & 0 & -6.577666 & 2.657115 & 2.235568 \\
\hline 71 & 1 & 0 & -5.723791 & 3.248590 & 2.598439 \\
\hline 72 & 1 & 0 & -7.415085 & 3.327141 & 2.026330 \\
\hline 73 & 1 & 0 & -6.875488 & 1.939890 & 3.014672 \\
\hline ro-poi & & & \multicolumn{3}{|c|}{0.574928 (Hartree/Particle) } \\
\hline ermal & 0 & & \multicolumn{3}{|c|}{0.615520} \\
\hline Thermal & to & & \multicolumn{3}{|c|}{0.616464} \\
\hline Thermal & 1 to & Energy= & \multicolumn{3}{|c|}{0.496148} \\
\hline Sum of $\mathrm{e}$ & and & Energies $=$ & \multicolumn{3}{|c|}{-2019.223930} \\
\hline Sum of $\mathrm{e}$ & and & nergies $=$ & \multicolumn{3}{|c|}{-2019.183338} \\
\hline Sum of $\mathrm{e}$ & & halpies= & \multirow{2}{*}{\multicolumn{3}{|c|}{-2019.102574}} \\
\hline & & & & & \\
\hline
\end{tabular}

M06 /6-311++G(d,p)-SDD/SMD//M06/6-31G(d)-LANL2DZ energy in toluene solvent $=-2021.4756426$

INT4da

\begin{tabular}{rrrrrr} 
Center & Atomic & Atomic & \multicolumn{2}{c}{ Coordinates (Angstroms) } \\
Number & Number & Type & X & Y & $Z$ \\
-1 & 6 & 0 & -2.762069 & 2.400034 & -2.290474 \\
2 & 6 & 0 & -2.515297 & 2.586963 & -0.931951 \\
3 & 6 & 0 & -1.642699 & 1.736578 & -0.256299 \\
4 & 6 & 0 & -1.025037 & 0.683064 & -0.929278 \\
5 & 6 & 0 & -1.256659 & 0.508580 & -2.292390 \\
6 & 6 & 0 & -2.126697 & 1.365718 & -2.970369 \\
7 & 1 & 0 & -3.001460 & 3.400892 & -0.394235 \\
8 & 1 & 0 & -1.450182 & 1.900786 & 0.806795 \\
9 & 1 & 0 & -0.763269 & -0.298231 & -2.839513 \\
10 & 1 & 0 & -2.307282 & 1.219069 & -4.035397 \\
11 & 53 & 0 & 1.664648 & -2.494020 & 1.499280 \\
12 & 46 & 0 & 0.113912 & -0.659349 & 0.114791 \\
13 & 15 & 0 & 1.886359 & 0.877140 & -0.086015 \\
14 & 6 & 0 & -1.097887 & -3.042270 & -1.487341 \\
15 & 1 & 0 & -0.272788 & -2.856405 & -2.177229 \\
16 & 6 & 0 & -1.275646 & -2.312046 & -0.405725 \\
17 & 6 & 0 & -1.862855 & -1.897500 & 0.735124 \\
18 & 1 & 0 & -1.464988 & -2.293126 & 1.674991 \\
19 & 6 & 0 & -2.026189 & -4.184874 & -1.755548 \\
20 & 8 & 0 & -3.049576 & -4.343150 & -1.123825 \\
21 & 6 & 0 & 2.816078 & 1.023309 & 1.475059 \\
22 & 6 & 0 & 4.203801 & 0.906581 & 1.530557 \\
23 & 6 & 0 & 2.091105 & 1.221443 & 2.655216 \\
24 & 6 & 0 & 4.860158 & 0.998275 & 2.754516 \\
25 & 1 & 0 & 4.775647 & 0.720077 & 0.622922
\end{tabular}




\begin{tabular}{|c|c|c|c|c|c|}
\hline 26 & 6 & 0 & 2.750440 & 1.327470 & 3.871959 \\
\hline 27 & 1 & 0 & 1.000367 & 1.267746 & 2.619397 \\
\hline 28 & 6 & 0 & 4.137690 & 1.213143 & 3.922059 \\
\hline 29 & 1 & 0 & 5.942803 & 0.892908 & 2.793839 \\
\hline 30 & 1 & 0 & 2.180138 & 1.479066 & 4.786463 \\
\hline 31 & 1 & 0 & 4.653737 & 1.279886 & 4.878252 \\
\hline 32 & 6 & 0 & 3.065749 & 0.350037 & -1.373702 \\
\hline 33 & 6 & 0 & 3.024645 & -0.955048 & -1.869466 \\
\hline 34 & 6 & 0 & 4.025141 & 1.244518 & -1.865091 \\
\hline 35 & 6 & 0 & 3.933898 & -1.362334 & -2.841438 \\
\hline 36 & 1 & 0 & 2.290933 & -1.657657 & -1.470160 \\
\hline 37 & 6 & 0 & 4.932520 & 0.832710 & -2.832998 \\
\hline 38 & 1 & 0 & 4.058957 & 2.267635 & -1.489964 \\
\hline 39 & 6 & 0 & 4.885817 & -0.470553 & -3.322696 \\
\hline 40 & 1 & 0 & 3.898877 & -2.382725 & -3.218356 \\
\hline 41 & 1 & 0 & 5.676582 & 1.532585 & -3.209331 \\
\hline 42 & 1 & 0 & 5.596468 & -0.791047 & -4.082743 \\
\hline 43 & 6 & 0 & 1.495528 & 2.603708 & -0.554175 \\
\hline 44 & 6 & 0 & 1.459146 & 3.633120 & 0.387936 \\
\hline 45 & 6 & 0 & 1.146619 & 2.879529 & -1.881866 \\
\hline 46 & 6 & 0 & 1.073261 & 4.916350 & 0.009124 \\
\hline 47 & 1 & 0 & 1.739206 & 3.440448 & 1.422451 \\
\hline 48 & 6 & 0 & 0.770756 & 4.160918 & -2.257282 \\
\hline 49 & 1 & 0 & 1.159060 & 2.082829 & -2.625353 \\
\hline 50 & 6 & 0 & 0.728501 & 5.182402 & -1.310351 \\
\hline 51 & 1 & 0 & 1.048847 & 5.711350 & 0.752484 \\
\hline 52 & 1 & 0 & 0.498697 & 4.361243 & -3.292165 \\
\hline 53 & 1 & 0 & 0.426726 & 6.186313 & -1.604080 \\
\hline 54 & 6 & 0 & -1.594385 & -5.107877 & -2.862825 \\
\hline 55 & 1 & 0 & -2.347568 & -5.885091 & -3.016932 \\
\hline 56 & 1 & 0 & -0.631229 & -5.571683 & -2.608907 \\
\hline 57 & 1 & 0 & -1.441288 & -4.547457 & -3.795221 \\
\hline 58 & 1 & 0 & -3.444018 & 3.064484 & -2.818823 \\
\hline 59 & 6 & 0 & -3.042213 & -1.032323 & 0.808944 \\
\hline 60 & 6 & 0 & -3.345581 & -0.406786 & 2.024241 \\
\hline 61 & 6 & 0 & -3.862138 & -0.818040 & -0.306204 \\
\hline 62 & 6 & 0 & -4.430258 & 0.449332 & 2.123273 \\
\hline 63 & 1 & 0 & -2.708467 & -0.586799 & 2.889892 \\
\hline 64 & 6 & 0 & -4.946460 & 0.036461 & -0.220933 \\
\hline 65 & 1 & 0 & -3.638060 & -1.329777 & -1.240045 \\
\hline 66 & 6 & 0 & -5.208422 & 0.663693 & 0.992547 \\
\hline 67 & 1 & 0 & -4.682196 & 0.954814 & 3.050689 \\
\hline 68 & 1 & 0 & -5.589086 & 0.231872 & -1.074071 \\
\hline 69 & 7 & 0 & -6.343228 & 1.589826 & 1.080898 \\
\hline 70 & 8 & 0 & -7.016545 & 1.755821 & 0.076593 \\
\hline 71 & 8 & 0 & -6.539685 & 2.140772 & 2.152632 \\
\hline \multicolumn{4}{|c|}{ Zero-point correction= } & \multicolumn{2}{|c|}{0.544911 (Hartree/Particle) } \\
\hline ermal & to & & & \multicolumn{2}{|c|}{0.585520} \\
\hline Thermal & to & & & \multicolumn{2}{|l|}{0.586464} \\
\hline Thermal & to & & & \multicolumn{2}{|c|}{0.465515} \\
\hline Sum of e & and & Energies $=$ & & \multirow{2}{*}{\multicolumn{2}{|c|}{-2109.203467}} \\
\hline Sum of e & and & ergies $=$ & & & -2109.162858 \\
\hline Sum of e & and & thalpies= & & \multicolumn{2}{|l|}{-2109.161914} \\
\hline Sum of & $\mathrm{c}$ and $\mathrm{t}$ & Energ & & \multicolumn{2}{|l|}{-2109.282863} \\
\hline
\end{tabular}

M06 /6-311++G(d,p)-SDD/SMD//M06/6-31G(d)-LANL2DZ energy in toluene solvent $=-2111.4514115$

INT4ea

\begin{tabular}{rrrrrr} 
Center & Atomic & Atomic & \multicolumn{2}{c}{ Coordinates (Angstroms) } \\
Number & Number & Type & X & Y & $Z$ \\
-1 & 6 & 0 & 0.625535 & 4.885315 & -0.439442 \\
2 & 6 & 0 & 0.079400 & 4.155540 & -1.492180 \\
3 & 6 & 0 & 0.106031 & 2.762766 & -1.466203 \\
4 & 6 & 0 & 0.691523 & 2.087183 & -0.394732 \\
5 & 6 & 0 & 1.229306 & 2.820319 & 0.661950 \\
6 & 6 & 0 & 1.195846 & 4.215738 & 0.638597 \\
7 & 1 & 0 & -0.381363 & 4.670847 & -2.334779 \\
8 & 1 & 0 & -0.343371 & 2.204275 & -2.291165 \\
9 & 1 & 0 & 1.687757 & 2.311582 & 1.512191 \\
10 & 1 & 0 & 1.624542 & 4.779160 & 1.467433 \\
11 & 53 & 0 & 1.064313 & -2.714707 & -0.571187 \\
12 & 46 & 0 & 0.851185 & 0.049841 & -0.442786 \\
13 & 15 & 0 & -1.414776 & -0.075336 & 0.194576 \\
14 & 6 & 0 & 3.672367 & 0.107213 & 0.876081 \\
15 & 1 & 0 & 3.123532 & 0.050012 & 1.817861 \\
16 & 6 & 0 & 3.057392 & 0.165361 & -0.289605 \\
17 & 6 & 0 & 2.939523 & 0.266311 & -1.623060 \\
18 & 1 & 0 & 2.861792 & -0.668928 & -2.185380 \\
19 & 6 & 0 & 5.163998 & 0.075112 & 0.901110 \\
20 & 8 & 0 & 5.834287 & 0.210777 & -0.101927 \\
21 & 6 & 0 & -2.327078 & -1.274398 & -0.831570 \\
22 & 6 & 0 & -3.150637 & -2.258494 & -0.287281 \\
23 & 6 & 0 & -2.151276 & -1.210439 & -2.217948 \\
24 & 6 & 0 & -3.792918 & -3.167412 & -1.123301 \\
25 & 1 & 0 & -3.275918 & -2.334514 & 0.791880 \\
26 & 6 & 0 & -2.805318 & -2.108447 & -3.049650 \\
27 & 1 & 0 & -1.473558 & -0.467161 & -2.643281 \\
28 & 6 & 0 & -3.624681 & -3.092143 & -2.501013 \\
29 & 1 & 0 & -4.422968 & -3.943120 & -0.691668 \\
30 & 1 & 0 & -2.658670 & -2.055028 & -4.126777 \\
31 & 1 & 0 & -4.123386 & -3.809026 & -3.150944
\end{tabular}




\begin{tabular}{|c|c|c|c|c|c|}
\hline 32 & 6 & 0 & -1.606937 & -0.590971 & 1.935377 \\
\hline 33 & 6 & 0 & -0.528761 & -1.152837 & 2.622624 \\
\hline 34 & 6 & 0 & -2.835117 & -0.432097 & 2.590672 \\
\hline 35 & 6 & 0 & -0.677890 & -1.558300 & 3.945405 \\
\hline 36 & 1 & 0 & 0.423375 & -1.290312 & 2.107803 \\
\hline 37 & 6 & 0 & -2.980611 & -0.842518 & 3.909705 \\
\hline 38 & 1 & 0 & -3.677372 & 0.020127 & 2.065894 \\
\hline 39 & 6 & 0 & -1.901685 & -1.405652 & 4.587661 \\
\hline 40 & 1 & 0 & 0.166034 & -2.000476 & 4.471547 \\
\hline 41 & 1 & 0 & -3.938977 & -0.719346 & 4.411332 \\
\hline 42 & 1 & 0 & -2.017429 & -1.724581 & 5.622282 \\
\hline 43 & 6 & 0 & -2.414813 & 1.455775 & 0.107808 \\
\hline 44 & 6 & 0 & -3.355152 & 1.675065 & -0.899400 \\
\hline 45 & 6 & 0 & -2.163561 & 2.469406 & 1.040029 \\
\hline 46 & 6 & 0 & -4.028582 & 2.892058 & -0.976604 \\
\hline 47 & 1 & 0 & -3.567688 & 0.894506 & -1.628713 \\
\hline 48 & 6 & 0 & -2.840688 & 3.677561 & 0.964881 \\
\hline 49 & 1 & 0 & -1.420352 & 2.317045 & 1.822843 \\
\hline 50 & 6 & 0 & -3.773378 & 3.893506 & -0.048009 \\
\hline 51 & 1 & 0 & -4.761634 & 3.051087 & -1.765862 \\
\hline 52 & 1 & 0 & -2.626502 & 4.460437 & 1.690461 \\
\hline 53 & 1 & 0 & -4.299307 & 4.844718 & -0.111913 \\
\hline 54 & 6 & 0 & 5.772320 & -0.134774 & 2.263347 \\
\hline 55 & 1 & 0 & 6.862954 & -0.104189 & 2.194953 \\
\hline 56 & 1 & 0 & 5.454722 & -1.104693 & 2.670410 \\
\hline 57 & 1 & 0 & 5.421529 & 0.634374 & 2.965039 \\
\hline 58 & 1 & 0 & 0.602939 & 5.973878 & -0.458390 \\
\hline 59 & 6 & 0 & 3.122150 & 1.537636 & -2.388486 \\
\hline 60 & 1 & 0 & 3.116807 & 2.415998 & -1.735558 \\
\hline 61 & 1 & 0 & 4.097858 & 1.480894 & -2.892176 \\
\hline 62 & 1 & 0 & 2.351062 & 1.662753 & -3.158068 \\
\hline \multicolumn{4}{|c|}{ Zero-noint correction= } & \multicolumn{2}{|c|}{0.488723 (Hartree/Particle) } \\
\hline (2) & to & & & \multicolumn{2}{|c|}{$\begin{array}{l}0.488723 \text { (Hartree/Partıcle) } \\
0.523909\end{array}$} \\
\hline Thermal & to & & & \multicolumn{2}{|c|}{0.524853} \\
\hline Thermal & to & Energy= & & \multicolumn{2}{|c|}{416443} \\
\hline Sum of e & and & Energies= & & \multicolumn{2}{|l|}{-1713.256725} \\
\hline Sum of e & and & nergies $=$ & & \multicolumn{2}{|c|}{-1713.221540} \\
\hline Sum of e & on & thalpies $=$ & & \multicolumn{2}{|l|}{-1713.220596} \\
\hline Sum of e & & e Energies & & \multicolumn{2}{|l|}{-1713.329005} \\
\hline
\end{tabular}

M06 /6-311++G(d,p)-SDD/SMD//M06/6-31G(d)-LANL2DZ energy in toluene solvent $=-1715.3379464$

INT4fa

\begin{tabular}{rrrrrr} 
Center & Atomic & Atomic & \multicolumn{2}{c}{ Coordinates (Angstroms) } \\
Number & Number & Type & X & Y & \\
$---------------r r r$ \\
1 & 6 & 0 & 0.782077 & 4.677673 & -0.141016 \\
2 & 6 & 0 & 0.700280 & 3.960143 & -1.331126 \\
3 & 6 & 0 & 0.671240 & 2.566702 & -1.312363 \\
4 & 6 & 0 & 0.739795 & 1.873277 & -0.103444 \\
5 & 6 & 0 & 0.807356 & 2.598425 & 1.087026 \\
6 & 6 & 0 & 0.831968 & 3.993793 & 1.069277 \\
7 & 1 & 0 & 0.644964 & 4.486012 & -2.284212 \\
8 & 1 & 0 & 0.574758 & 2.025719 & -2.256287 \\
9 & 1 & 0 & 0.821711 & 2.078338 & 2.048471 \\
10 & 1 & 0 & 0.884881 & 4.544687 & 2.008530 \\
11 & 53 & 0 & 0.384053 & -2.902621 & 0.546451 \\
12 & 46 & 0 & 0.678979 & -0.176589 & 0.021979 \\
13 & 15 & 0 & -1.666524 & 0.087508 & 0.029629 \\
14 & 6 & 0 & 3.438298 & 0.550654 & 1.442364 \\
15 & 1 & 0 & 2.857689 & 1.171618 & 2.124305 \\
16 & 6 & 0 & 2.894736 & -0.068308 & 0.408946 \\
17 & 6 & 0 & 2.950409 & -0.838061 & -0.686904 \\
18 & 1 & 0 & 2.776663 & -1.911716 & -0.551298 \\
19 & 6 & 0 & 4.901944 & 0.400879 & 1.674916 \\
20 & 8 & 0 & 5.639573 & -0.151770 & 0.881316 \\
21 & 6 & 0 & -2.507755 & -1.246430 & -0.891272 \\
22 & 6 & 0 & -3.653075 & -1.882951 & -0.415675 \\
23 & 6 & 0 & -1.961585 & -1.637314 & -2.118102 \\
24 & 6 & 0 & -4.247950 & -2.895125 & -1.163525 \\
25 & 1 & 0 & -4.073379 & -1.607355 & 0.550312 \\
26 & 6 & 0 & -2.566252 & -2.635730 & -2.869143 \\
27 & 1 & 0 & -1.038131 & -1.172795 & -2.469582 \\
28 & 6 & 0 & -3.709835 & -3.269010 & -2.389186 \\
29 & 1 & 0 & -5.133706 & -3.397842 & -0.779056 \\
30 & 1 & 0 & -2.130926 & -2.937341 & -3.820009 \\
31 & 1 & 0 & -4.175573 & -4.064624 & -2.968168 \\
32 & 6 & 0 & -2.337239 & 0.094899 & 1.725137 \\
33 & 6 & 0 & -1.529177 & -0.321426 & 2.785638 \\
34 & 6 & 0 & -3.649223 & 0.513671 & 1.983001 \\
35 & 6 & 0 & -2.026429 & -0.325242 & 4.085137 \\
36 & 1 & 0 & -0.511543 & -0.658206 & 2.583127 \\
37 & 6 & 0 & -4.143397 & 0.504299 & 3.281403 \\
38 & 1 & 0 & -4.280815 & 0.857605 & 1.163175 \\
39 & 6 & 0 & -3.331354 & 0.085471 & 4.333065 \\
40 & 1 & 0 & -1.390047 & -0.653944 & 4.904551 \\
41 & 1 & 0 & -5.164564 & 0.828717 & 3.474089 \\
42 & 1 & 0 & -3.719432 & 0.082121 & 5.350354 \\
43 & 6 & 0 & -2.348678 & 1.617403 & -0.717604 \\
44 & 6 & 0 & -2.702169 & 1.652134 & -2.068897 \\
45 & 6 & 0 & -2.405048 & 2.798098 & 0.030429 \\
46 & 6 & 0 & -3.097549 & 2.846875 & -2.662186
\end{tabular}




$\begin{array}{rrrrrr}47 & 1 & 0 & -2.678305 & 0.741926 & -2.666335 \\ 48 & 6 & 0 & -2.802613 & 3.988510 & -0.564245 \\ 49 & 1 & 0 & -2.123782 & 2.792309 & 1.082652 \\ 50 & 6 & 0 & -3.145192 & 4.016933 & -1.913335 \\ 51 & 1 & 0 & -3.374430 & 2.858570 & -3.715105 \\ 52 & 1 & 0 & -2.832604 & 4.900861 & 0.029354 \\ 53 & 1 & 0 & -3.452958 & 4.951824 & -2.379034 \\ 54 & 6 & 0 & 5.406891 & 0.995841 & 2.963140 \\ 55 & 1 & 0 & 4.910847 & 0.518313 & 3.819339 \\ 56 & 1 & 0 & 5.162518 & 2.065912 & 3.010767 \\ 57 & 1 & 0 & 6.488783 & 0.859845 & 3.041521 \\ 58 & 1 & 0 & 0.799658 & 5.766417 & -0.157477 \\ 59 & 6 & 0 & 3.383107 & -0.414796 & -2.071860 \\ 60 & 6 & 0 & 2.255848 & -0.684174 & -3.068892 \\ 61 & 1 & 0 & 1.909678 & -1.726122 & -3.005590 \\ 62 & 1 & 0 & 1.392934 & -0.030162 & -2.875284 \\ 63 & 1 & 0 & 2.606397 & -0.496699 & -4.094093 \\ 64 & 6 & 0 & 3.830181 & 1.040293 & -2.115682 \\ 65 & 1 & 0 & 4.677332 & 1.200718 & -1.435742 \\ 66 & 1 & 0 & 4.154275 & 1.298334 & -3.133606 \\ 67 & 1 & 0 & 3.023867 & 1.727743 & -1.829070 \\ 68 & 6 & 0 & 4.578626 & -1.323417 & -2.396026 \\ 69 & 1 & 0 & 5.375892 & -1.193791 & -1.653097 \\ 70 & 1 & 0 & 4.285621 & -2.382398 & -2.411484 \\ 71 & 1 & 0 & 4.975756 & -1.064968 & -3.387868\end{array}$

Zero-point correction=

Thermal correction to Energy=

0.574068 (Hartree/Particle)

Thermal correction to Enthalpy=

0.612726

Thermal correction to Gibbs Free Energy=

Sum of electronic and zero-point Energies=

Sum of electronic and thermal Energies=

Sum of electronic and thermal Enthalpies=

0.499746

$-1831.017572$

$-1831.017572$

$-1830.978914$

$-1831.091894$

M06 /6-311++G(d,p)-SDD/SMD//M06/6-31G(d)-LANL2DZ energy in toluene solvent $=-1833.2155732$

INT4ga

\begin{tabular}{|c|c|c|c|c|c|}
\hline \multirow{2}{*}{$\begin{array}{l}\text { Center } \\
\text { Number }\end{array}$} & \multirow{2}{*}{$\begin{array}{l}\text { Atomic } \\
\text { Number }\end{array}$} & \multirow{2}{*}{$\begin{array}{l}\text { Atomic } \\
\text { Type }\end{array}$} & \multicolumn{3}{|c|}{ Coordinates (Angstroms) } \\
\hline & & & $\mathrm{X}$ & $\mathrm{Y}$ & Z \\
\hline 1 & 6 & 0 & 0.976432 & 4.585537 & 0.606068 \\
\hline 2 & 6 & 0 & 0.845067 & 4.075719 & -0.682924 \\
\hline 3 & 6 & 0 & 0.755216 & 2.701161 & -0.892181 \\
\hline 4 & 6 & 0 & 0.803255 & 1.827116 & 0.191263 \\
\hline 5 & 6 & 0 & 0.917859 & 2.338151 & 1.483968 \\
\hline 6 & 6 & 0 & 1.011008 & 3.715380 & 1.690468 \\
\hline 7 & 1 & 0 & 0.807632 & 4.751690 & -1.536874 \\
\hline 8 & 1 & 0 & 0.643386 & 2.316317 & -1.906305 \\
\hline 9 & 1 & 0 & 0.914560 & 1.669274 & 2.349036 \\
\hline 10 & 1 & 0 & 1.104803 & 4.105462 & 2.703998 \\
\hline 11 & 53 & 0 & 0.407068 & -2.974361 & 0.276697 \\
\hline 12 & 46 & 0 & 0.677890 & -0.214860 & 0.021336 \\
\hline 13 & 15 & 0 & -1.655434 & 0.105454 & 0.052147 \\
\hline 14 & 6 & 0 & 3.504676 & -0.003832 & 1.458001 \\
\hline 15 & 1 & 0 & 2.924983 & 0.368589 & 2.302509 \\
\hline 16 & 6 & 0 & 2.939140 & -0.280868 & 0.307352 \\
\hline 17 & 6 & 0 & 2.740049 & -0.640327 & -0.964798 \\
\hline 18 & 1 & 0 & 2.580912 & -1.690075 & -1.223386 \\
\hline 19 & 6 & 0 & 4.984433 & -0.196747 & 1.615689 \\
\hline 20 & 8 & 0 & 5.688713 & -0.553795 & 0.697846 \\
\hline 21 & 6 & 0 & -2.364997 & -1.027588 & -1.186560 \\
\hline 22 & 6 & 0 & -3.463756 & -1.841197 & -0.916659 \\
\hline 23 & 6 & 0 & -1.725443 & -1.113875 & -2.428651 \\
\hline 24 & 6 & 0 & -3.920709 & -2.732262 & -1.883224 \\
\hline 25 & 1 & 0 & -3.949863 & -1.801843 & 0.057214 \\
\hline 26 & 6 & 0 & -2.193599 & -1.993776 & -3.394293 \\
\hline 27 & 1 & 0 & -0.835832 & -0.509880 & -2.624161 \\
\hline 28 & 6 & 0 & -3.289641 & -2.808217 & -3.119429 \\
\hline 29 & 1 & 0 & -4.769153 & -3.377153 & -1.661637 \\
\hline 30 & 1 & 0 & -1.688167 & -2.061819 & -4.355758 \\
\hline 31 & 1 & 0 & -3.645142 & -3.512914 & -3.869148 \\
\hline 32 & 6 & 0 & -2.453273 & -0.227822 & 1.654880 \\
\hline 33 & 6 & 0 & -1.731931 & -0.848832 & 2.676487 \\
\hline 34 & 6 & 0 & -3.787427 & 0.139664 & 1.875073 \\
\hline 35 & 6 & 0 & -2.338428 & -1.105037 & 3.902298 \\
\hline 36 & 1 & 0 & -0.699685 & -1.151736 & 2.496750 \\
\hline 37 & 6 & 0 & -4.389848 & -0.122544 & 3.098791 \\
\hline 38 & 1 & 0 & -4.350840 & 0.640044 & 1.086866 \\
\hline 39 & 6 & 0 & -3.664481 & -0.744081 & 4.113131 \\
\hline 40 & 1 & 0 & -1.771335 & -1.593427 & 4.692384 \\
\hline 41 & 1 & 0 & -5.427439 & 0.162507 & 3.263590 \\
\hline 42 & 1 & 0 & -4.137217 & -0.944267 & 5.073355 \\
\hline 43 & 6 & 0 & -2.285556 & 1.759850 & -0.409945 \\
\hline 44 & 6 & 0 & -2.641313 & 2.060101 & -1.726640 \\
\hline 45 & 6 & 0 & -2.296183 & 2.778628 & 0.549844 \\
\hline 46 & 6 & 0 & -2.993553 & 3.359710 & -2.078774 \\
\hline 47 & 1 & 0 & -2.648485 & 1.278900 & -2.485148 \\
\hline 48 & 6 & 0 & -2.647182 & 4.073368 & 0.194449 \\
\hline 49 & 1 & 0 & -2.010859 & 2.562863 & 1.579134 \\
\hline 50 & 6 & 0 & -2.992949 & 4.368037 & -1.122305 \\
\hline 51 & 1 & 0 & -3.271027 & 3.580973 & -3.108056 \\
\hline 52 & 1 & 0 & -2.640581 & 4.858421 & 0.948782 \\
\hline
\end{tabular}




$\begin{array}{rrrrrr}53 & 1 & 0 & -3.263093 & 5.385354 & -1.400685 \\ 54 & 6 & 0 & 5.511238 & 0.092947 & 2.994833 \\ 55 & 1 & 0 & 6.593925 & -0.054703 & 3.018544 \\ 56 & 1 & 0 & 5.031226 & -0.567268 & 3.730268 \\ 57 & 1 & 0 & 5.270035 & 1.124501 & 3.286151 \\ 58 & 1 & 0 & 1.045731 & 5.660839 & 0.764340 \\ 59 & 6 & 0 & 3.015724 & 0.267261 & -2.130594 \\ 60 & 9 & 0 & 1.923833 & 0.425372 & -2.897435 \\ 61 & 9 & 0 & 3.436991 & 1.470171 & -1.765075 \\ 62 & 9 & 0 & 3.951709 & -0.291228 & -2.898245\end{array}$

Zero-point correction=

0.466598 (Hartree/Particle)

Thermal correction to Energy=

0.503511

Thermal correction to Enthalpy=

0.504455

Thermal correction to Gibbs Free Energy=

Sum of electronic and zero-point Energies=

Sum of electronic and thermal Energies=

0.392832

$-2010.925502$

$-2010.888589$

Sum of electronic and thermal Enthalpies=

$-2010.887645$

Sum of electronic and thermal Free Energies $=\quad-2010.999267$

M06 /6-311++G(d,p)-SDD/SMD//M06/6-31G(d)-LANL2DZ energy in toluene solvent $=-2013.0332871$

INT4ha

\begin{tabular}{|c|c|c|c|c|c|}
\hline \multirow{2}{*}{$\begin{array}{l}\text { Center } \\
\text { Number }\end{array}$} & \multirow{2}{*}{$\begin{array}{l}\text { Atomic } \\
\text { Number }\end{array}$} & \multirow{2}{*}{$\begin{array}{l}\text { Atomic } \\
\text { Type }\end{array}$} & \multicolumn{3}{|c|}{ Coordinates (Angstroms) } \\
\hline & & & $\mathrm{X}$ & $\mathrm{Y}$ & \\
\hline 1 & 6 & 0 & 0.194862 & 4.933693 & -0.552309 \\
\hline 2 & 6 & 0 & -0.372331 & 4.145547 & -1.549896 \\
\hline 3 & 6 & 0 & -0.199658 & 2.762715 & -1.535182 \\
\hline 4 & 6 & 0 & 0.532557 & 2.154331 & -0.514554 \\
\hline 5 & 6 & 0 & 1.110152 & 2.946915 & 0.476679 \\
\hline 6 & 6 & 0 & 0.937792 & 4.332089 & 0.458234 \\
\hline 7 & 1 & 0 & -0.959316 & 4.606110 & -2.344416 \\
\hline 8 & 1 & 0 & -0.668917 & 2.158958 & -2.315710 \\
\hline 9 & 1 & 0 & 1.694991 & 2.495374 & 1.279812 \\
\hline 10 & 1 & 0 & 1.391163 & 4.940998 & 1.240426 \\
\hline 11 & 53 & 0 & 1.322517 & -2.605215 & -0.660479 \\
\hline 12 & 46 & 0 & 0.825754 & 0.128596 & -0.532876 \\
\hline 13 & 15 & 0 & -1.403782 & -0.165483 & 0.219634 \\
\hline 14 & 6 & 0 & 3.823102 & 0.369402 & 0.487604 \\
\hline 15 & 6 & 0 & 3.077422 & 0.439210 & -0.597390 \\
\hline 16 & 6 & 0 & 2.657009 & 0.565771 & -1.860733 \\
\hline 17 & 1 & 0 & 2.652820 & -0.299340 & -2.526070 \\
\hline 18 & 6 & 0 & 5.301602 & 0.549941 & 0.260318 \\
\hline 19 & 8 & 0 & 5.756207 & 0.861744 & -0.820277 \\
\hline 20 & 6 & 0 & -2.315666 & -1.320644 & -0.859681 \\
\hline 21 & 6 & 0 & -3.354011 & -2.121095 & -0.382015 \\
\hline 22 & 6 & 0 & -2.008450 & -1.328706 & -2.223033 \\
\hline 23 & 6 & 0 & -4.078347 & -2.918943 & -1.262045 \\
\hline 24 & 1 & 0 & -3.593462 & -2.132231 & 0.680905 \\
\hline 25 & 6 & 0 & -2.740172 & -2.118446 & -3.100878 \\
\hline 26 & 1 & 0 & -1.178520 & -0.722335 & -2.591256 \\
\hline 27 & 6 & 0 & -3.775148 & -2.915665 & -2.619553 \\
\hline 28 & 1 & 0 & -4.880504 & -3.549722 & -0.882854 \\
\hline 29 & 1 & 0 & -2.491278 & -2.123247 & -4.160361 \\
\hline 30 & 1 & 0 & -4.342768 & -3.543030 & -3.304736 \\
\hline 31 & 6 & 0 & -1.575982 & -0.787244 & 1.930732 \\
\hline 32 & 6 & 0 & -0.534280 & -1.508227 & 2.520019 \\
\hline 33 & 6 & 0 & -2.757450 & -0.572923 & 2.653521 \\
\hline 34 & 6 & 0 & -0.673282 & -2.010964 & 3.809703 \\
\hline 35 & 1 & 0 & 0.377018 & -1.698635 & 1.952165 \\
\hline 36 & 6 & 0 & -2.892382 & -1.080605 & 3.939913 \\
\hline 37 & 1 & 0 & -3.573000 & -0.002564 & 2.208644 \\
\hline 38 & 6 & 0 & -1.849475 & -1.798062 & 4.520216 \\
\hline 39 & 1 & 0 & 0.143453 & -2.573933 & 4.257677 \\
\hline 40 & 1 & 0 & -3.815012 & -0.911199 & 4.492544 \\
\hline 41 & 1 & 0 & -1.956445 & -2.192370 & 5.529529 \\
\hline 42 & 6 & 0 & -2.457985 & 1.331423 & 0.206811 \\
\hline 43 & 6 & 0 & -3.429558 & 1.537725 & -0.773307 \\
\hline 44 & 6 & 0 & -2.203296 & 2.342978 & 1.140344 \\
\hline 45 & 6 & 0 & -4.130266 & 2.740650 & -0.823257 \\
\hline 46 & 1 & 0 & -3.640218 & 0.759558 & -1.506165 \\
\hline 47 & 6 & 0 & -2.901779 & 3.539830 & 1.087374 \\
\hline 48 & 1 & 0 & -1.439731 & 2.198753 & 1.905141 \\
\hline 49 & 6 & 0 & -3.866567 & 3.742589 & 0.102143 \\
\hline 50 & 1 & 0 & -4.887659 & 2.889872 & -1.591208 \\
\hline 51 & 1 & 0 & -2.685532 & 4.322561 & 1.812469 \\
\hline 52 & 1 & 0 & -4.411423 & 4.684232 & 0.058115 \\
\hline 53 & 6 & 0 & 6.193353 & 0.319421 & 1.454721 \\
\hline 54 & 1 & 0 & 6.012247 & -0.664990 & 1.905922 \\
\hline 55 & 1 & 0 & 6.002847 & 1.068999 & 2.234808 \\
\hline 56 & 1 & 0 & 7.236264 & 0.392711 & 1.135320 \\
\hline 57 & 1 & 0 & 2.465886 & 1.550729 & -2.288493 \\
\hline 58 & 1 & 0 & 0.059523 & 6.014161 & -0.564466 \\
\hline 59 & 6 & 0 & 3.286628 & 0.077143 & 1.857917 \\
\hline 60 & 1 & 0 & 3.554782 & -0.939953 & 2.178576 \\
\hline 61 & 1 & 0 & 3.679868 & 0.779349 & 2.605584 \\
\hline 62 & 1 & 0 & 2.191571 & 0.141375 & 1.868964 \\
\hline
\end{tabular}

Zero-point correction

0.489804 (Hartree/Particle)

Thermal correction to Energy=

0.524410
0.525354

Thermal correction to Enthalpy=

Thermal correction to Gibbs Free Energy=

0.419881 
Sum of electronic and zero-point Energies= Sum of electronic and thermal Energies= Sum of electronic and thermal Enthalpies=

Sum of electronic and thermal Free Energies

$-1713.256116$

$-1713.221510$

$-1713.220566$

$-1713.326039$

M06 /6-311++G(d,p)-SDD/SMD//M06/6-31G(d)-LANL2DZ energy in toluene solvent = -1715.3368901

INT4ia

\begin{tabular}{|c|c|c|c|c|c|}
\hline \multirow{2}{*}{$\begin{array}{l}\text { Center } \\
\text { Number }\end{array}$} & \multirow{2}{*}{$\begin{array}{l}\text { Atomic } \\
\text { Number }\end{array}$} & \multirow{2}{*}{$\begin{array}{c}\text { Atomic } \\
\text { Type }\end{array}$} & \multicolumn{3}{|c|}{ Coordinates (Angstroms) } \\
\hline & & & $\mathrm{X}$ & $\mathrm{Y}$ & $\mathrm{Z}$ \\
\hline 1 & 6 & 0 & -0.121679 & 4.833225 & -1.139155 \\
\hline 2 & 6 & 0 & -0.713016 & 4.032525 & -2.112290 \\
\hline 3 & 6 & 0 & -0.599085 & 2.644114 & -2.044439 \\
\hline 4 & 6 & 0 & 0.108288 & 2.047588 & -1.000480 \\
\hline 5 & 6 & 0 & 0.688258 & 2.850788 & -0.019757 \\
\hline 6 & 6 & 0 & 0.575420 & 4.239807 & -0.091212 \\
\hline 7 & 1 & 0 & -1.265736 & 4.489256 & -2.933019 \\
\hline 8 & 1 & 0 & -1.078952 & 2.029451 & -2.810115 \\
\hline 9 & 1 & 0 & 1.218437 & 2.393743 & 0.818391 \\
\hline 10 & 1 & 0 & 1.035192 & 4.858841 & 0.679619 \\
\hline 11 & 53 & 0 & 0.666948 & -2.740674 & -0.792445 \\
\hline 12 & 46 & 0 & 0.353795 & 0.018305 & -0.916070 \\
\hline 13 & 15 & 0 & -1.700410 & -0.080773 & 0.244010 \\
\hline 14 & 6 & 0 & 3.556550 & 0.277395 & -0.751995 \\
\hline 15 & 6 & 0 & 2.513149 & 0.214729 & -1.557684 \\
\hline 16 & 6 & 0 & 1.803310 & 0.186289 & -2.692326 \\
\hline 17 & 1 & 0 & 1.631469 & -0.758692 & -3.209917 \\
\hline 18 & 6 & 0 & 4.896757 & 0.390497 & -1.449046 \\
\hline 19 & 8 & 0 & 4.983829 & 0.532680 & -2.649534 \\
\hline 20 & 6 & 0 & -2.737322 & -1.410583 & -0.448967 \\
\hline 21 & 6 & 0 & -3.329459 & -2.400307 & 0.333159 \\
\hline 22 & 6 & 0 & -2.889753 & -1.446836 & -1.839512 \\
\hline 23 & 6 & 0 & -4.074076 & -3.410077 & -0.270070 \\
\hline 24 & 1 & 0 & -3.191026 & -2.402493 & 1.413270 \\
\hline 25 & 6 & 0 & -3.644413 & -2.446772 & -2.436700 \\
\hline 26 & 1 & 0 & -2.389815 & -0.697487 & -2.457423 \\
\hline 27 & 6 & 0 & -4.234981 & -3.433090 & -1.650364 \\
\hline 28 & 1 & 0 & -4.522179 & -4.188393 & 0.345011 \\
\hline 29 & 1 & 0 & -3.753539 & -2.470272 & -3.519357 \\
\hline 30 & 1 & 0 & -4.811536 & -4.228712 & -2.119065 \\
\hline 31 & 6 & 0 & -1.485212 & -0.391828 & 2.026186 \\
\hline 32 & 6 & 0 & -0.210624 & -0.637885 & 2.539972 \\
\hline 33 & 6 & 0 & -2.584440 & -0.341588 & 2.893994 \\
\hline 34 & 6 & 0 & -0.040543 & -0.846779 & 3.905688 \\
\hline 35 & 1 & 0 & 0.651203 & -0.674319 & 1.869762 \\
\hline 36 & 6 & 0 & -2.410296 & -0.561406 & 4.253934 \\
\hline 37 & 1 & 0 & -3.577389 & -0.121581 & 2.499841 \\
\hline 38 & 6 & 0 & -1.136562 & -0.814056 & 4.759559 \\
\hline 39 & 1 & 0 & 0.958248 & -1.033161 & 4.297044 \\
\hline 40 & 1 & 0 & -3.268919 & -0.528728 & 4.922349 \\
\hline 41 & 1 & 0 & -1.000203 & -0.980275 & 5.827085 \\
\hline 42 & 6 & 0 & -2.781275 & 1.398775 & 0.223432 \\
\hline 43 & 6 & 0 & -3.903593 & 1.495691 & -0.600967 \\
\hline 44 & 6 & 0 & -2.420885 & 2.493474 & 1.017529 \\
\hline 45 & 6 & 0 & -4.647106 & 2.672417 & -0.636403 \\
\hline 46 & 1 & 0 & -4.208036 & 0.648835 & -1.214100 \\
\hline 47 & 6 & 0 & -3.166342 & 3.663290 & 0.982462 \\
\hline 48 & 1 & 0 & -1.546387 & 2.431515 & 1.665048 \\
\hline 49 & 6 & 0 & -4.279203 & 3.757461 & 0.149861 \\
\hline 50 & 1 & 0 & -5.522829 & 2.734520 & -1.280460 \\
\hline 51 & 1 & 0 & -2.870532 & 4.509471 & 1.600471 \\
\hline 52 & 1 & 0 & -4.861659 & 4.676866 & 0.119405 \\
\hline 53 & 6 & 0 & 6.112028 & 0.305658 & -0.563382 \\
\hline 54 & 1 & 0 & 6.115653 & -0.635805 & 0.002169 \\
\hline 55 & 1 & 0 & 6.111261 & 1.112278 & 0.181430 \\
\hline 56 & 1 & 0 & 7.010112 & 0.371187 & -1.183203 \\
\hline 57 & 1 & 0 & 1.513530 & 1.113027 & -3.188856 \\
\hline 58 & 1 & 0 & -0.209012 & 5.917225 & -1.195466 \\
\hline 59 & 6 & 0 & 3.488930 & 0.216112 & 0.732074 \\
\hline 60 & 6 & 0 & 3.562856 & 1.390928 & 1.485506 \\
\hline 61 & 6 & 0 & 3.415216 & -1.014361 & 1.390095 \\
\hline 62 & 6 & 0 & 3.544938 & 1.341446 & 2.875588 \\
\hline 63 & 1 & 0 & 3.635177 & 2.348861 & 0.968027 \\
\hline 64 & 6 & 0 & 3.411317 & -1.063240 & 2.781192 \\
\hline 65 & 1 & 0 & 3.342787 & -1.929177 & 0.801731 \\
\hline 66 & 6 & 0 & 3.474160 & 0.112023 & 3.525390 \\
\hline 67 & 1 & 0 & 3.595667 & 2.263507 & 3.452359 \\
\hline 68 & 1 & 0 & 3.353161 & -2.028181 & 3.283702 \\
\hline 69 & 1 & 0 & 3.470653 & 0.069994 & 4.613985 \\
\hline Zero-poin & rrection= & & & .541237 (Hart & ree/Particle) \\
\hline Thermal & rection to $\mathrm{E}$ & $\mathrm{ggy}=$ & & 579272 & \\
\hline Thermal & rection to $\mathrm{E}$ & alpy= & & 580216 & \\
\hline Thermal & rection to $\mathrm{G}$ & os Free Energy= & & 67095 & \\
\hline Sum of el & tronic and $\mathrm{z}$ & -point Energies= & & 1904.790761 & \\
\hline Sum of el & tronic and th & mal Energies= & & -1904.752726 & \\
\hline Sum of el & tronic and th & mal Enthalpies= & & 1904.751782 & \\
\hline Sum of el & tronic and th & mal Free Energies= & & 904.864904 & \\
\hline
\end{tabular}




\begin{tabular}{|c|c|c|c|c|c|}
\hline \multirow{2}{*}{$\begin{array}{l}\text { Center } \\
\text { Number }\end{array}$} & \multirow{2}{*}{$\begin{array}{l}\text { Atomic } \\
\text { Number }\end{array}$} & \multirow{2}{*}{$\begin{array}{l}\text { Atomic } \\
\text { Type }\end{array}$} & \multicolumn{3}{|c|}{ Coordinates (Angstroms) } \\
\hline & & & $\mathrm{X}$ & Y & $\mathrm{Z}$ \\
\hline 1 & 6 & 0 & 0.204362 & 4.900706 & -0.581323 \\
\hline 2 & 6 & 0 & -0.398560 & 4.165940 & -1.598132 \\
\hline 3 & 6 & 0 & -0.306379 & 2.775375 & -1.606370 \\
\hline 4 & 6 & 0 & 0.381857 & 2.111489 & -0.590904 \\
\hline 5 & 6 & 0 & 1.000360 & 2.847547 & 0.418315 \\
\hline 6 & 6 & 0 & 0.906616 & 4.240654 & 0.422195 \\
\hline 7 & 1 & 0 & -0.952121 & 4.673848 & -2.387728 \\
\hline 8 & 1 & 0 & -0.803506 & 2.212943 & -2.400003 \\
\hline 9 & 1 & 0 & 1.553535 & 2.344017 & 1.212117 \\
\hline 10 & 1 & 0 & 1.389749 & 4.808623 & 1.217116 \\
\hline 11 & 53 & 0 & 0.811715 & -2.696883 & -0.619058 \\
\hline 12 & 46 & 0 & 0.545054 & 0.066277 & -0.592813 \\
\hline 13 & 15 & 0 & -1.702568 & -0.071983 & 0.183830 \\
\hline 14 & 6 & 0 & 3.719885 & 0.217224 & -0.119124 \\
\hline 15 & 6 & 0 & 2.693734 & 0.221157 & -0.953888 \\
\hline 16 & 6 & 0 & 2.169139 & 0.305797 & -2.188587 \\
\hline 17 & 1 & 0 & 2.035142 & -0.591672 & -2.793288 \\
\hline 18 & 6 & 0 & 5.090044 & 0.326074 & -0.754690 \\
\hline 19 & 8 & 0 & 5.195915 & 0.511764 & -1.947484 \\
\hline 20 & 6 & 0 & -2.630889 & -1.278879 & -0.819692 \\
\hline 21 & 6 & 0 & -3.438436 & -2.261507 & -0.248801 \\
\hline 22 & 6 & 0 & -2.487424 & -1.226405 & -2.210345 \\
\hline 23 & 6 & 0 & -4.098780 & -3.177947 & -1.062199 \\
\hline 24 & 1 & 0 & -3.538182 & -2.328428 & 0.833581 \\
\hline 25 & 6 & 0 & -3.159147 & -2.132019 & -3.019329 \\
\hline 26 & 1 & 0 & -1.823635 & -0.483748 & -2.657935 \\
\hline 27 & 6 & 0 & -3.963679 & -3.112915 & -2.443971 \\
\hline 28 & 1 & 0 & -4.716509 & -3.951509 & -0.609488 \\
\hline 29 & 1 & 0 & -3.039024 & -2.086037 & -4.100097 \\
\hline 30 & 1 & 0 & -4.477079 & -3.835030 & -3.076396 \\
\hline 31 & 6 & 0 & -1.876111 & -0.584978 & 1.925897 \\
\hline 32 & 6 & 0 & -0.813688 & -1.206391 & 2.585419 \\
\hline 33 & 6 & 0 & -3.085822 & -0.379147 & 2.603081 \\
\hline 34 & 6 & 0 & -0.959589 & -1.619324 & 3.906336 \\
\hline 35 & 1 & 0 & 0.122550 & -1.383145 & 2.057012 \\
\hline 36 & 6 & 0 & -3.227387 & -0.798492 & 3.919435 \\
\hline 37 & 1 & 0 & -3.917128 & 0.112298 & 2.096578 \\
\hline 38 & 6 & 0 & -2.163314 & -1.417296 & 4.572057 \\
\hline 39 & 1 & 0 & -0.126982 & -2.104055 & 4.412402 \\
\hline 40 & 1 & 0 & -4.170929 & -0.639317 & 4.438739 \\
\hline 41 & 1 & 0 & -2.275296 & -1.741887 & 5.605335 \\
\hline 42 & 6 & 0 & -2.686500 & 1.467261 & 0.093205 \\
\hline 43 & 6 & 0 & -3.637772 & 1.682895 & -0.904589 \\
\hline 44 & 6 & 0 & -2.382713 & 2.503217 & 0.984514 \\
\hline 45 & 6 & 0 & -4.264966 & 2.921348 & -1.018356 \\
\hline 46 & 1 & 0 & -3.891395 & 0.883686 & -1.599811 \\
\hline 47 & 6 & 0 & -3.007089 & 3.736090 & 0.866667 \\
\hline 48 & 1 & 0 & -1.634214 & 2.351483 & 1.762746 \\
\hline 49 & 6 & 0 & -3.947273 & 3.949573 & -0.139684 \\
\hline 50 & 1 & 0 & -5.007697 & 3.077896 & -1.799051 \\
\hline 51 & 1 & 0 & -2.750267 & 4.538119 & 1.556562 \\
\hline 52 & 1 & 0 & -4.431604 & 4.919935 & -0.236654 \\
\hline 53 & 6 & 0 & 6.297790 & 0.181811 & 0.131675 \\
\hline 54 & 1 & 0 & 6.304836 & -0.801618 & 0.616793 \\
\hline 55 & 1 & 0 & 6.297182 & 0.932231 & 0.930612 \\
\hline 56 & 1 & 0 & 7.193009 & 0.294998 & -0.484602 \\
\hline 57 & 1 & 0 & 1.994674 & 1.279536 & -2.647355 \\
\hline 58 & 1 & 0 & 0.130796 & 5.987191 & -0.573910 \\
\hline 59 & 6 & 0 & 3.572984 & 0.093673 & 1.365894 \\
\hline 60 & 9 & 0 & 4.151646 & 1.131834 & 1.994137 \\
\hline 61 & 9 & 0 & 4.152169 & -1.017282 & 1.830834 \\
\hline 62 & 9 & 0 & 2.296676 & 0.072796 & 1.766183 \\
\hline \multicolumn{6}{|c|}{0.467033 (Hartree/Particle) } \\
\hline \multicolumn{3}{|c|}{ Thermal correction to Energy= } & \multicolumn{2}{|c|}{0.503560} & \\
\hline \multicolumn{3}{|c|}{ Thermal correction to Enthalpy= } & \multicolumn{2}{|c|}{0.504504} & \\
\hline Thermal & rrection to $\mathrm{C}$ & os Free Energy= & 0.3 & 94571 & \\
\hline Sum of $\mathrm{e}$ & tronic and $\mathrm{z}$ & -point Energies= & & 2010.930723 & \\
\hline Sum of $\mathrm{e}$ & tronic and $\mathrm{tl}$ & mal Energies= & & -2010.894196 & \\
\hline Sum of e & ctronic and $\mathrm{tl}$ & mal Enthalpies= & & 2010.893251 & \\
\hline Sum of $\mathrm{e}$ & tronic and $\mathrm{tl}$ & mal Free Energies= & & 2011.003185 & \\
\hline
\end{tabular}

